Technische Universität München

Lehrstuhl für Mikrotechnik und Medizingerätetechnik

Univ.-Prof. Dr. rer. nat. Tim Christian Lüth

\title{
Mikrofertigung und Mikromontage zur Herstellung eines individuellen piezoelektrisch betriebenen Mikrotropfenerzeugers
}

\author{
Markus Albert Kagerer
}

Vollständiger Abdruck der von der Fakultät für Maschinenwesen der Technischen Universität München zur Erlangung des akademischen Grades eines

Doktor-Ingenieurs (Dr.-Ing.)

genehmigten Dissertation.

Vorsitzender: $\quad$ Univ.-Prof. Dr.-Ing. Wolfram Volk

Prüfer der Dissertation: $\quad$ 1. Univ.-Prof. Dr. rer. nat. Tim Christian Lüth

2. Univ.-Prof. Dr.-Ing. Norbert Schwesinger

Die Dissertation wurde am 26.08.2015 bei der Technischen Universität München eingereicht und durch die Fakultät für Maschinenwesen am 27.07.2016 angenommen. 


\section{Vorwort}

Die vorliegende Arbeit aus dem Bereich der Mikrosystemtechnik entstand während meiner Zeit als wissenschaftlicher Mitarbeiter am Lehrstuhl für Mikrotechnik und Medizingerätetechnik der Technischen Universität München.

Mein besonderer Dank gilt meinem Doktorvater Herrn Prof. Dr. rer. nat. Tim C. Lüth, Ordinarius des Lehrstuhles für Mikrotechnik und Medizingerätetechnik an der Technischen Universität München, für die intensive Betreuung, die wohlwollende Förderung und das mir entgegengebrachten Vertrauen. Des Weiteren danke ich Herrn Prof. Dr. rer. nat. Tim C. Lüth für die geistigen Freiräume und die gezielten Anregungen, welche entscheidend zum Gelingen dieser Arbeit beigetragen haben.

Meinem Zweitprüfer Herrn Prof. Dr.-Ing. Norbert Schwesinger, Leiter des Fachgebietes Mikrostrukturierte Mechatronische Systeme an der Technischen Universität München, danke ich für die Betreuung dieser Arbeit als Zweitgutachter und vor allem dafür, dass er mich mit seinen Lehrveranstaltungen schon als Student für die Mikrosystemtechnik begeisterte.

Bei Herrn Prof. Dr.-Ing. Wolfram Volk, Ordinarius des Lehrstuhles für Umformtechnik und Gießereiwesen an der Technischen Universität München, bedanke ich mich für die Übernahme des Vorsitzes.

Danken möchte ich zudem der Bayerischen Forschungsstiftung, deren Fördermittel die finanzielle Basis für die vorliegenden Forschungstätigkeiten geschaffen haben.

Meinen Kollegen danke ich für das sehr angenehme Arbeitsklima, für die vielen zielführenden Diskussionen und vor allem für das kollegiale Arbeitsklima am Lehrstuhl. Hierbei möchte ich mich besonders bei Herrn Dr.-Ing. Franz Irlinger, Herrn Dr.-Ing Thomas Ottnad und dem gesamten Werkstattteam unter der Leitung von Herrn Gerhard Ribnitzky bedanken.

Besonderen Dank möchte ich an dieser Stelle auch den vielen Studenten aussprechen, die im Rahmen ihrer Abschlussarbeiten oder auch ihrer HiWi-Tätigkeiten entscheidend zum Gelingen dieser Arbeit beigetragen haben. Spezielle Dankesworte richte ich hierbei an Herrn Dipl.-Ing. Dominik Rumschöttel, Herrn M. Sc. Arne Meeuw und Herrn Dipl.-Ing. Kenji Lars Eiler. Vor allem freut es mich sehr, dass Herr Dipl.-Ing. Dominik Rumschöttel nach seinem Studium den Weg in die Gruppe Mikrotechnik am Lehrstuhl gefunden hat.

Auch möchte ich mich an dieser Stelle bei Herrn Peter Werndl, Oberlandesgerichtspräsident a. D., Herrn Dipl.-Ing. Dominik Rumschöttel, Herrn M. Sc. Markus Menacher und Frau Volljuristin Monika Werndl für die Durchsicht der Arbeit bedanken.

Ganz besonderer Dank gilt meiner Familie und meinen engen Freunden, die mir im Laufe der Jahre den notwendigen Rückhalt gegeben haben, um vorliegende Arbeit anzufertigen.

Nicht zuletzt möchte ich meiner Lebensgefährtin Volljuristin Monika Werndl für ihre moralische Unterstützung und ihren liebevollen und ununterbrochenen Beistand für die Fertigstellung der Dissertation danken. 


\section{Kurzfassung}

Mikrosysteme sind aus unserem Alltag nicht mehr wegzudenken. Sie sorgen dafür, dass Ärzte während Operationen unterstützt werden, Autos sicherer und energiesparender fahren oder auch Fotos zuhause gedruckt werden können. Die Systeme besitzen kleine Massen, ermöglichen schnelle Reaktionen und erlauben den Zusammenschluss unterschiedlicher Funktionen platzsparend in einem dreidimensionalen Aufbau meist mit hybrider Architektur. Für ihre Fertigung werden Verfahren sowohl für die Mikrostrukturierung von Substraten als auch für die Mikromontage von Funktionskomponenten bzw. -werkstoffen eingesetzt, welche aus der Mikroelektronik hervorgehen. Diese zeichnen sich vor allem durch ihre kostengünstige Fertigung von hohen Stückzahlen eines identischen Produktes aus. Die konstant steigende Komplexität von Mikrosystemen erfordert jedoch bereits in frühen Phasen ihrer Entwicklung eine Funktionsprüfung an realen und individuellen Funktionsmodellen.

Die vorliegende Arbeit löst dabei die Aufgabe der kostengünstigen Herstellung von individuellen Funktionsmodellen für die Mikrosystemtechnik in geringen Stückzahlen und gleichzeitig auch in kurzer Zeit, indem an diese Randbedingungen angepasste Fertigungstechniken entwickelt, optimiert und eingesetzt werden.

Für die Mikrostrukturierung von Substraten werden vorliegend sowohl die Laserstrahlmaterialbearbeitung als auch das Trennschleifen mit einer Wafersäge eingesetzt. Die Laserstrahlmaterialbearbeitung zeichnet sich dadurch aus, dass es nahezu keine Einschränkung in der Formenvielfalt gibt und dass vor allem keine aufwendige und teure Photolithographie für die Strukturübertragung gefordert ist. Im Rahmen dieser Arbeit ist hierzu das Zusammenspiel der grundlegenden Bearbeitungsparameter Fokusposition, Vorschubgeschwindigkeit und auch Linienabstand sowohl auf das Laserstrahlschneiden als auch auf den ebenenweisen Laserstrahlmaterialabtrag aufgezeigt. Diese Parameter sind nicht von der Laserstrahlquelle abhängig, so dass die aufgezeigten Zusammenhänge auf jedes Lasersystem übertragen werden können. Für die Erstellung von senkrechten Kanälen mit geringer Rauheit an den Seitenwänden für die Mikrofluidik wird vorliegend das Trennschleifen mit einer Wafersäge eingesetzt. Für die notwendige Fügung von zwei Bauteilen über eine Klebschicht ist ein Mikromontageplatz erstellt, mit dem sowohl Klebschichten auf Substraten als auch Relativpositionierungen von zwei Bauteilen durchgeführt werden können.

Die untersuchten Fertigungsverfahren werden erfolgreich bei der Herstellung von piezoelektrisch betriebenen Mikrotropfenerzeugern als Anwendungsbeispiel eingesetzt. Es werden insgesamt fünf identische Mikrotropfenerzeuger gleichzeitig in einem Batchverfahren gefertigt, so dass eine ausreichend hohe Anzahl an Funktionsmodellen in der Produktentstehung für die Eigenschaftsbewertung vorliegt. Diese werden in weniger als 10 Stunden gefertigt.

Zusammenfassend gilt, dass durch die vorliegende Arbeit individuelle Funktionsmodelle eines Mikrosystems in geringen Stückzahlen und gleichzeitig in kurzer Zeit fertigbar sind. Es steht ein kostengünstiger Fertigungsprozess mit einer angepassten Genauigkeit zur Verfügung. Es kann in frühen Phasen der Produktentstehung eines Mikrosystems auf notwendige Änderungen mit einem Redesign reagiert werden. 


\section{Inhaltsverzeichnis}

Symbolverzeichnis ..................................................................................................................... VI

1. Problemstellung ......................................................................................................................... 1

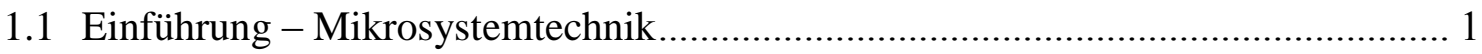

1.2 Anwendungsbeispiel aus der Mikrosystemtechnik - Mikrotropfenerzeugung......... 2

1.2.1 Tropfendosierung mit Drop-on-Demand Techniken ................................. 2

1.2.2 Unterteilung piezoelektrisch betriebener Mikrotropfenerzeuger................. 3

1.2.3 Einsatz piezoelektrisch betriebener Mikrotropfenerzeuger ........................ 5

1.3 Problembeschreibung / Anwendung - Fertigung von Mikrosystemen ..................... 7

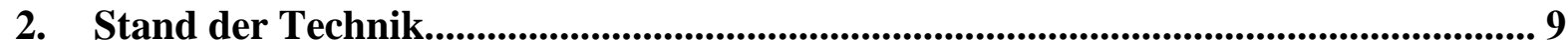

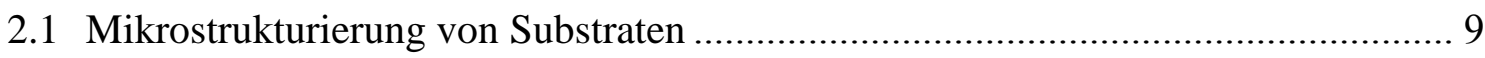

2.1.1 Prozessablaufplan für die Mikrostrukturierung von Substraten ................ 10

2.1.2 Basisprozesse für die Mikrostrukturierung von Substraten ....................... 12

2.1.3 Reinheit in der Mikrostrukturierung von Substraten ............................... 15

2.2 Mikromontage in der Mikrosystemtechnik ........................................................... 17

2.2.1 Mikromontage im Vergleich zu standardisierten Montagetechniken ........ 17

2.2.2 Prozessablauf in der Mikromontage ..................................................... 19

2.2.3 Gerätschaften für die Mikromontage ..................................................... 19

2.2.4 Klebstoffauftragsverfahren in Mikromontageanlagen .............................. 23

2.3 Zusammenfassende Betrachtung des Stands der Technik ................................... 25

3. Kritik am Stand der Technik............................................................................. 26

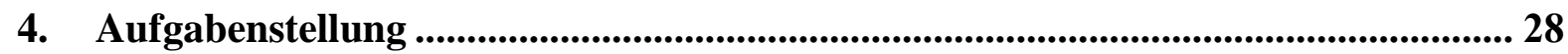

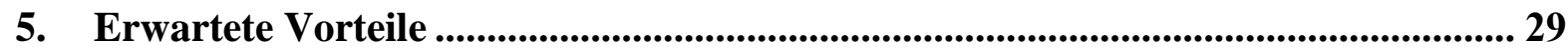

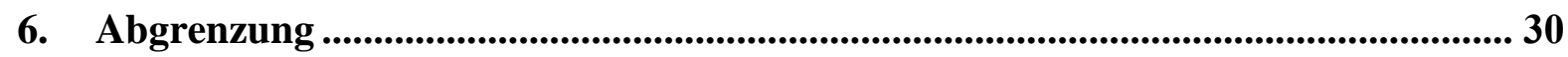

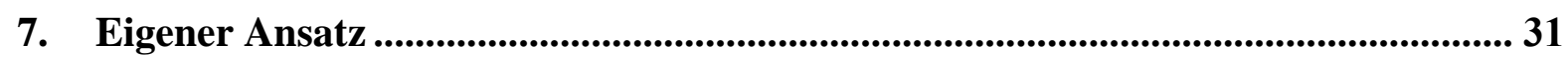

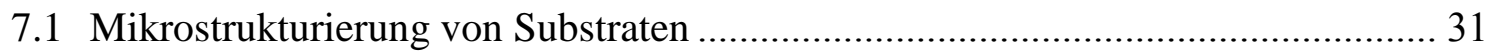

7.1.1 Werkzeuge für die Strukturierung von Substraten................................. 31

7.1.2 Grundlagen der Laserstrahlmaterialbearbeitung ................................... 33

7.1.3 Gaußsche Strahlenoptik ............................................................... 35

7.1.4 Laserstrahlquellen unabhängige Parameter für die Materialbearbeitung .. 37

7.1.5 Fertigung senkrechter Kanäle mithilfe des Trennschleifens ...................... 41

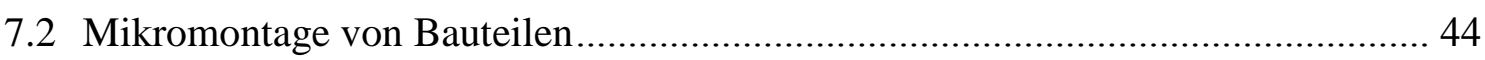

7.2.1 Aufbau des Mikromontageplatzes ........................................................... 44

7.2.2 Ausrichten und Absetzen von zwei Bauteilen ......................................... 47

7.2.3 Definierter Klebstoffauftrag auf planare Bauteile ................................... 51

7.2.4 Lösungsprozess für die Fügung zweier Bauteile ..................................... 53

7.3 Zusammenfassende Betrachtung des eigenen Ansatzes ...................................... 55

8. Unterscheidungsmerkmale zum Stand der Technik .............................................. 56 


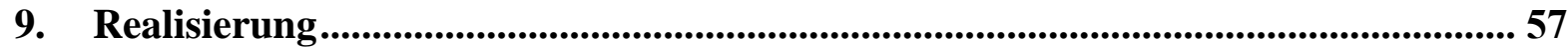

9.1 Werkzeuge für die Strukturierung von Substraten .......................................... 57

9.1.1 Laserstrahlmaterialbearbeitung mit Nanosekunden Laserstrahlquellen .... 57

9.1.2 Fertigung senkrechter Kanäle mit einer Wafersäge ................................ 59

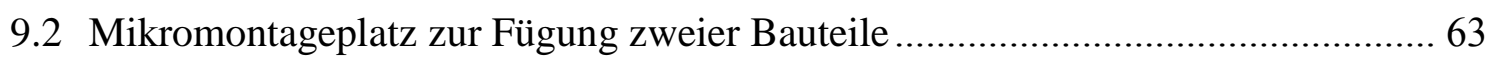

9.2.1 Gestell des Mikromontageplatzes .................................................... 63

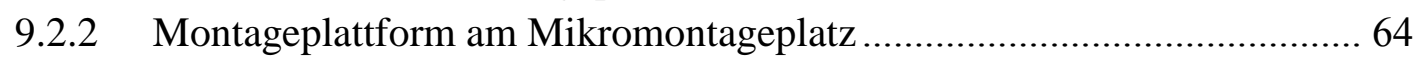

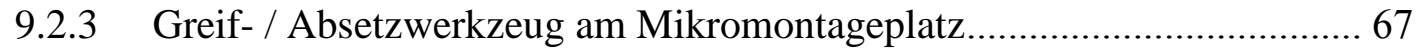

9.2.4 Klebstoffauftragswerkzeug am Mikromontageplatz................................ 69

9.2.5 Gesamtaufbau des Mikromontageplatzes ........................................... 71

9.2.6 Anwendung des Mikromontageplatzes............................................... 72

9.3 Zusammenfassende Betrachtung der Realisierung ............................................... 75

10. Experimente zur Mikrostrukturierung und zur Mikromontage ............................ 76

10.1 Experiment 1: Laserstrahlschneiden in Silizium............................................... 76

10.2 Experiment 2: Ebener Laserstrahlmaterialabtrag in Silizium .............................. 80

10.3 Experiment 3: Fertigung senkrechter Kanäle mithilfe des Trennschleifens ........... 84

10.4Experiment 4: Positionierung zweier Bauteile mit dem Mikromontageplatz ......... 86

10.5 Experiment 5: Klebstoffauftrag mit dem Mikromontageplatz ............................. 89

11. Bedeutung der Ergebnisse ............................................................................................ 92

11.1 Beschreibung eines planaren piezoelektrischen Mikrotropfenerzeugers ................ 92

11.1.1 Aufbau des Mikrotropfenerzeugers ................................................... 92

11.1.2 Funktionsweise des Mikrotropfenerzeugers ............................................. 94

11.2 Herstellung individueller Funktionsmodelle des Mikrotropfenerzeugers................ 95

11.2.1 Prozessflow zur Herstellung der Mikrotropfenerzeuger ........................... 95

11.2.2 Schritt 1: Mikrostrukturierung mittels Laserstrahlung ............................. 96

11.2.3 Schritt 2: Mikrostrukturierung mittels Trennschleifen ............................. 96

11.2.4 Schritt 3: Verbindung von Membran und Substrat ................................... 97

11.2.5 Schritt 4: Mikromontage von piezoelektrischer Keramik und Membran .. 97

11.2.6 Schritt 5: Strukturierung der Elektroden mittels Trennschleifen ............... 98

11.2.7 Schritt 6: Vereinzelung mittels Trennschleifen ....................................... 98

11.2.8 Bewertung und Gegenüberstellung des Prozesses ................................... 99

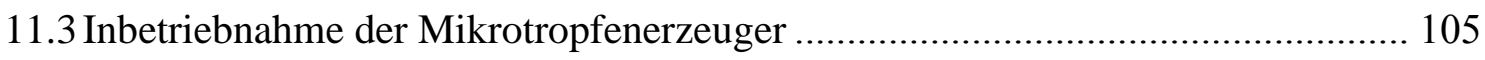

11.3.1 Oberflächenmodifikation der Düsenplatte ........................................... 106

11.3.2 Montage der Druckchips auf eine Platine ............................................. 108

11.3.3 Aufnahmevorrichtung für platinenbasierte Druckchips......................... 109

11.3.4 Kontrolle von Düsenfunktionalität und Hub des bimorphen Aufbaus .... 111

11.4Fluidmechanisches Dosierexperiment / -beispiel........................................... 114

12. Zusammenfassung und Ausblick................................................................................ 119

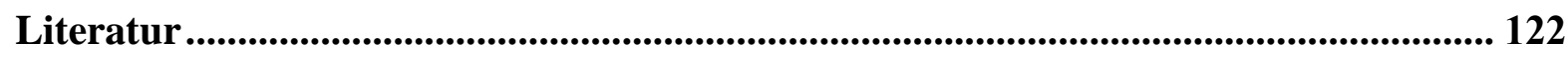

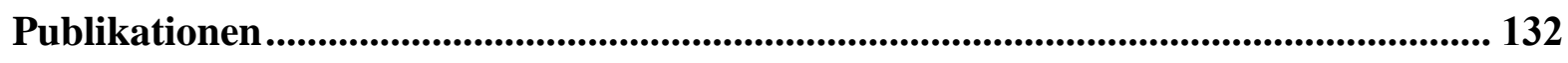

Betreute studentische Arbeiten ................................................................................... 134 


\section{Symbolverzeichnis}

\section{Symbol}

a

A

Ani

$\mathrm{a}_{\text {oben }}$

$\mathrm{a}_{\text {unten }}$

b

$\mathrm{b}_{\text {Sub }}$

d

D

$\mathrm{d}_{\text {Austritt }}$

$\mathrm{d}_{\text {Eintritt }}$

$\mathrm{d}_{\text {Haupt }}$

$\mathrm{d}_{\text {Satellit }}$

$\mathrm{d}_{\text {Shape }}$

$\mathrm{d}_{\text {Soll }}$

$\mathrm{d}_{\mathrm{w}}$

$\mathrm{E}_{\mathrm{Kin}}$

$\mathrm{E}_{\text {Oberf }}$

$\mathrm{E}_{\text {Visk }}$

f

$\mathrm{Fl}_{\text {Ober }}$

$\mathrm{Fl}_{\text {Boden }}$

$\mathrm{F}_{\mathrm{A}}$

$\mathrm{F}_{\mathrm{H}}$

$\mathrm{F}_{\mathrm{S}}$

$f_{\text {Puls }}$

g

$\mathrm{I}(\mathrm{r}, \mathrm{z})$

$\mathrm{I}_{0}(0)$

$\mathrm{I}_{0}(\mathrm{z})$

\section{Bedeutung}

Beschleunigung des Werkstückes in Bewegungsrichtung

Fläche des Druckunterschiedes

Grad der Anisotropie

Abstand Positionierung obere Ecke

Abstand Positionierung untere Ecke

Geforderte Kanalbreite

Breite des Testsubstrates

Sägeblattbreite

Durchmesser des Laserstrahles auf der Fokussieroptik

Durchmesser an der Laserstrahlaustrittsseite

Durchmesser an der Laserstrahleintrittsseite

Durchmesser des Haupttropfens

Durchmesser des Satellitentropfens

Dimension / Durchmesser der Schneidform

Sollmaß / Durchmesser nach Laserprozess

Bearbeitungsdurchmesser

Energie für Tropfenflug

Energie für Tropfenformung

Energie für Überwindung der Reibung im Düsenkanal

Brennweite der Fokussieroptik

Fläche auf der Oberseite des Ablationsgebietes

Fläche auf dem Boden des Ablationsgebietes

Abreißkraft

Haltekraft

Ansaugkraft

Pulsfrequenz

Erdbeschleunigung

Örtliche Intensitätsverteilung des Laserstrahles

Maximale Intensität $(r=0 ; z=0)$

Maximale Intensität auf der Strahlachse $(r=0)$

\section{SI-Einheit}

$\left[\mathrm{m} / \mathrm{s}^{2}\right]$

$\left[\mathrm{m}^{2}\right]$

[-]

[m]

[m]

[m]

[m]

[m]

[m]

[m]

[m]

[m]

[m]

[m]

[m]

[m]

[J]

[J]

[J]

[m]

$\left[\mathrm{m}^{2}\right]$

$\left[\mathrm{m}^{2}\right]$

[N]

[N]

[N]

[Hz]

$\left[\mathrm{m} / \mathrm{s}^{2}\right]$

[W/m $\left.\mathrm{m}^{2}\right]$

[W/m $\left.\mathrm{m}^{2}\right]$

$\left[\mathrm{W} / \mathrm{m}^{2}\right]$ 


\section{Symbol}

1

$1_{\text {Düse }}$

$\mathrm{m}$

$\mathrm{M}^{2}$

n

$\mathrm{P}_{\text {Mittel }}$

$\mathrm{P}_{\text {Puls }}$

PÜ

$\mathrm{r}_{\text {Kurb }}$

$\mathrm{R}_{\mathrm{a}}$

$\mathrm{R}_{\text {Lat }}$

$\mathrm{r}_{\text {Tropf }}$

$\mathrm{R}_{\mathrm{Ver}}$

$r_{\text {Wobbel }}$

$\mathrm{R}_{\mathrm{z}}$

S

SÜ

$\mathrm{T}$

$\mathrm{T}_{\mathrm{abt}}$

$\mathrm{t}_{\text {Puls }}$

$\mathrm{V}_{\mathrm{abt}}$

$\mathrm{V}_{\text {Düse }}$

$\mathrm{V}_{\text {Fluid }}$

$\mathrm{v}_{\mathrm{s}}$

$\mathbf{v}_{\text {Tropf }}$

$\mathrm{w}(\mathrm{z})$

$\mathrm{w}_{0}$

$\mathrm{X}_{\text {Kolb }}$

$\mathrm{z}_{\mathrm{R}}$

$\alpha$

$\beta$

$\gamma$

$\Delta \mathrm{p}$

$\Delta \mathrm{v}$

\section{Bedeutung}

Länge des Pleuels

Länge der Düse

Masse des Werkstïckes

Beugungsmaßzahl

Anzahl

Mittlere Laserleistung im Pulsbetrieb

Laserpulsspitzenleistung

Pulsüberlapp

Kurbelradius

Mittlere Rauheit

Laterale Ätzrate

Radius des ausgestoßenen Tropfens

Vertikale Ätzrate

Wobbelradius

Gemittelte Rauhtiefe

Sicherheitsfaktor

Spurüberlapp

Waferdicke

Abladierte Tiefe nach dem Laserprozess

Pulsperiodendauer

Abladiertes Volumen nach dem Laserprozess

Fluidgeschwindigkeit in der Düse

Ausgestoßenes Fluidvolumen

Vorschubgeschwindigkeit des Laserspots

Tropfenfluggeschwindigkeit

Strahlradius als Funktion der Entfernung z von Fokus

Fokusradius

Kolbenweg

Rayleigh Länge

Flankenwinkel

Stangenwinkel

Winkelversatz

Druckdifferenz (Umgebungsdruck / Unterdruck)

Versatz der Sägeschnitte

\section{SI-Einheit}

[m]

[m]

$[\mathrm{kg}]$

$[-]$

$[-]$

[W]

[W]

[\%]

[m]

[m]

$[\mathrm{m} / \mathrm{s}]$

[m]

$[\mathrm{m} / \mathrm{s}]$

[m]

[m]

[-]

[\%]

[m]

[m]

[s]

$\left[\mathrm{m}^{3}\right]$

$[\mathrm{m} / \mathrm{s}]$

$\left[\mathrm{m}^{3}\right]$

$[\mathrm{m} / \mathrm{s}]$

$[\mathrm{m} / \mathrm{s}]$

[m]

[m]

[m]

[m]

$\left[{ }^{\circ}\right]$

$\left[{ }^{\circ}\right]$

[ $\left.{ }^{\circ}\right]$

[Pa]

[m] 


\section{Symbol}

$\Delta \mathrm{y}$

$\eta$

$\theta_{\text {Kont }}$

$\theta_{\text {Kurb }}$

$\theta_{\text {Real }}$

$\lambda$

$\lambda_{\text {Schub }}$

$\rho$

$\sigma$

$\tau_{\text {Puls }}$

\section{Bedeutung}

Linienabstand

Viskosität der Flüssigkeit

Kontaktwinkel

Kurbelwinkel

Realer Divergenzwinkel

Wellenlänge

Schubstangenverhältnis

Dichte der Flüssigkeit

Oberflächenspannung der Flüssigkeit

Pulsdauer

\section{SI-Einheit}

[m]

$[\mathrm{Pa} \cdot \mathrm{s}]$

$\left[{ }^{\circ}\right]$

[ $\left.{ }^{\circ}\right]$

$\left[{ }^{\circ}\right]$

[m]

[-]

$\left[\mathrm{kg} / \mathrm{m}^{3}\right]$

$[\mathrm{N} / \mathrm{m}]$

[s] 


\section{Problemstellung}

\subsection{Einführung - Mikrosystemtechnik}

Die Mikrosystemtechnik umfasst den Entwurf, die Anwendung und die Fertigung von Mikrosystemen. Damit ein System als Mikrosystem bezeichnet werden kann, im englischen Sprachraum Micro-Electro-Mechanical-System (MEMS) genannt, kommt es entscheidend auf die Größe der in ihm enthaltenen funktionstragenden Strukturen an, die die eigentliche Aufgabe der Sensierung, der Aktuierung oder auch der Datenverarbeitung übernehmen. Diese müssen kleiner als einen Millimeter aber größer als einen Mikrometer sein (Gad-el-Hak, 2006). Die Gemeinsamkeit von Mikrosystemen ist, dass unterschiedliche Materialien, Technologien und Funktionen in einem System miteinander verbunden sind (Schwesinger, Dehne und Adler, 2009). Mikrosysteme vereinigen somit je nach ihrer Anwendung Komponenten aus den physikalischen Domänen Mechanik, Elektronik, Fluidik und Optik.

Die Mikrosystemtechnik geht aus der Mikroelektronik hervor, die ihren Anfang mit der Entwicklung des Transistors in den 1940er Jahren bei den „Bell Telephone Laboratories“ hatte (Bardeen und Brattain, 1948). Mit der Entdeckung des piezoresistiven Effektes in Germanium und in Silizium (Smith, 1954) wurde der Grundstein gelegt, um Materialien aus der Mikroelektronik nicht nur für elektronische Systeme, sondern auch für Mikrosysteme zu nutzen. Die Verbindung mehrerer Domänen in einem System bei kleiner Baugröße führt dazu, dass intelligente Systeme entstehen, die aus dem Alltag nicht mehr wegzudenken sind. Die Systeme besitzen kleine Massen, ermöglichen schnelle Reaktionen und erlauben den Zusammenschluss unterschiedlicher Funktionen platzsparend in einem Aufbau (Hilleringmann, 2006).

Die Mikrosystemtechnik ist eine der Schlüsseltechnologien des 21. Jahrhunderts (Globisch, 2011). Die Branche der Mikrosystemtechnik beschäftigte im Jahr 2009 ca. 766.000 Personen bei einem Umsatzvolumen von mehr als 82 Milliarden Euro in Deutschland (Bundesministerium für Bildung und Forschung, 2014). Nahezu unwahrnehmbar durchdringen Mikrosysteme unseren Alltag. Sie sorgen dafür, dass Ärzte während Operationen unterstützt werden, Autos sicherer und energiesparender fahren, Telefone zu universalen Kommunikationszentren werden oder auch Fotos zuhause gedruckt werden können. Beispiele für Mikrosysteme in diesem Zusammenhang sind Mikrogreifer in der Medizintechnik, Beschleunigungssensoren zur Auslösung von Airbags im Auto, Positionssensoren in Smartphones zur Navigation oder auch Tropfenerzeuger in Tintenstrahldruckern.

Vor allem mikrofluidische Systeme, wie bspw. Mikrotropfenerzeuger, zählen zu den erfolgreichsten Mikrosystemen (Hartzell, da Silva und Shea, 2011). Dies ist nicht nur allein ihrer Verwendung im umsatzstarken Markt der „Home and Office“ Tintenstrahldrucktechnik geschuldet, sondern insbesondere auch ihrer Eigenschaft, eine weitere Vielzahl an Fluiden mit unterschiedlichen Eigenschaften mit Volumina bis hinab in den Pikoliter Bereich zu dosieren. Daraus ergeben sich kontinuierlich neue Anwendungsfelder für Tropfenerzeuger.

Nachfolgend wird ein Überblick zu piezoelektrisch betriebenen Mikrotropfenerzeugern als ein Anwendungsbeispiel aus der Mikrosystemtechnik gegeben. Hierbei werden sowohl ihre möglichen Bauformen als auch deren Einsatzgebiete aufgezeigt. Es wird dargelegt, in welcher Vielfalt ein Mikrosystem für eine konkrete Aufgabe, hier der Tropfenabgabe, vorliegen kann. 


\subsection{Anwendungsbeispiel aus der Mikrosystemtechnik - Mikrotropfenerzeugung}

\subsubsection{Tropfendosierung mit Drop-on-Demand Techniken}

Eines der größten Anwendungsgebiete für Mikrosysteme ist die Tintenstrahldrucktechnik im „Home and Office“ Bereich. Hierbei werden einzelne Tropfen auf das Substrat bzw. Papier platziert. Diese Art des Dosierens wird als Drop-on-Demand (DoD) Technik bezeichnet, da nur auf Abruf ein Tropfen aus dem Druckkopf ausgestoßen wird. Im Unterschied hierzu wird bei der Continuous Jet Technik ein kontinuierlicher Tropfenstrahl erzeugt. Continuous Jets werden hauptsächlich in industriellen Anwendungen mit hohem Durchsatz, wie bspw. dem Drucken von Barcodes, eingesetzt.

DoD Systeme werden nach ihrem Aktuationsprinzip unterschieden. Dabei haben sich das Bubble Jet und das Piezo Jet Verfahren etabliert (Wijshoff, 2010). Ihre Gemeinsamkeit besteht in der Art der Tropfenerzeugung. Durch das Verringern des Volumens einer fluidgefüllten Kammer entsteht ein Druckimpuls, welcher einen Tropfen aus der Düse ausstößt.

Die Bubble Jet Technik nutzt hierfür als Aktor Heizelemente in den Fluidkammern. Das Fluid wird über seine Siedetemperatur erhitzt, so dass abrupt eine Gasblase entsteht, welche wiederum das Kammervolumen verringert und den Tropfenausstoß initiiert. Einer der wichtigsten Vorteile thermisch betriebener Tropfenerzeuger ist die Möglichkeit, dichte Tropfenerzeugerarrays zu erstellen, da als Aktor ein einfacher und schmaler Heizwiderstand ausreichend ist (Madou, 2002; Tseng, 2006). Auf der anderen Seite können allerdings nur thermisch stabile Fluide eingesetzt werden (Scheicher, 2004). Die Zyklen des abrupten Aufheizens und des Abkühlens ändern die chemische Zusammensetzung des Arbeitsfluides (Lee, 2003).

Piezoelektrisch betriebene Mikrotropfenerzeuger nutzen hingegen den inversen piezoelektrischen Effekt zur Tropfengenerierung. Dies bedeutet, dass mit Anlegen eines elektrischen Feldes eine mechanische Deformation an einer piezoelektrischen Keramik hervorgerufen wird. Durch die konstruktive Auslegung des piezoelektrischen Aktors wird hierdurch ebenfalls das Volumen einer fluidgefüllten Kammer verringert. Die am häufigsten eingesetzte piezoelektrische Keramik ist aufgrund ihres hohen Wirkungsgrades Blei Zirkonat Titanat $\mathrm{Pb}\left(\mathrm{Zr}_{\mathrm{x}} \mathrm{Ti}_{1-\mathrm{x}}\right) \mathrm{O}_{3}$ (Wijshoff, 2010). Die Vorteile piezoelektrisch aktuierter Tropfenerzeuger gegenüber thermisch aktuierten Systemen sind, dass die chemische Zusammensetzung des Arbeitsfluides über der Betriebszeit nicht negativ beeinflusst wird und dass das System ohne Verzögerungen auf Änderungen der elektrischen Ansteuerung über die reaktionsschnelle piezoelektrische Keramik reagiert (Tseng, 2006; Wijshoff, 2010). Ihr Nachteil liegt jedoch in der vergleichsweisen komplexen Herstellung (Lee, 2003). Die Integration der piezoelektrischen Aktoren gestaltet sich schwieriger als die Integration von Heizwiderständen bei thermisch betriebenen Systemen. Grund hierfür sind die geringen Längungen der piezoelektrischen Keramiken im Promille Bereich, so dass deren Positionierung bezogen auf die Fluidkammer und auch deren Aktorbauweise einen erheblichen Einfluss auf den entstehenden Druckimpuls besitzen. Allerdings ist dieses Verhalten, die chemische Zusammensetzung des Arbeitsfluides während des Betriebes nicht zu verändern, unabdingbar für die Erschließung neuer Anwendungsgebiete (Lee, 2003).

Es werden nachfolgend sowohl die Unterteilung als auch Anwendungsbeispiele von piezoelektrisch betriebenen Tropfenerzeugern vorgestellt. Dabei wird sich zeigen, dass das Einsatzspektrum von piezoelektrisch betriebenen Systemen weit über die Tintenstrahldrucktechnik hinaus reicht. 


\subsubsection{Unterteilung piezoelektrisch betriebener Mikrotropfenerzeuger}

In Abhängigkeit der Aktorbauform bzw. -anordnung werden piezoelektrisch betriebene Mikrotropfenerzeuger in fünf verschiedene Typen unterteilt: Scherung (,Shear"), Biegefinger (,Paddle“), Quetschung (,Squeeze“), Drücken („Push“) und Biegemembran („Bend“).

In Mikrotropfenerzeugern, die nach dem Shear Modus arbeiten, wird der piezoelektrische Schereffekt genutzt. Die piezoelektrischen Aktoren sind in Lamellenform angeordnet. Das Anlegen eines elektrischen Feldes senkrecht zu der Polarisationsrichtung führt zu einer Scherung der Lamellen. Die Kammer zwischen zwei Lamellen stellt das zu verringernde Fluidvolumen dar (siehe Abb. 1 (a)). Durch das Anlegen eines elektrischen Impulses wird das Volumen in der Kammer verringert. Als Ergebnis wird durch den resultierenden Druckimpuls ein Tropfen aus der Düse ausgestoßen. Das Fluid ist aufgrund der Bauweise dieses Typus in Kontakt mit den piezoelektrischen Lamellen. Durch das Prinzip der Volumenverringerung durch zwei Lamellen kann nur gleichzeitig aus jeder dritten Düse ein Tropfen ausgestoßen werden. Der Shear Modus findet kommerziell u. a. in Druckköpfen der Firmen XAAR, Spectra ${ }^{\mathrm{TM}}$ (FUJIFILM), ToshibaTEC, Konica und Sharp Anwendung (Wijshoff, 2010). Die Mikrotropfenerzeuger bestehen aus einer polarisierten piezoelektrischen Basisplatte mit Elektroden, einer unpolarisierten piezoelektrischen Abdeckplatte und einer Düsenplatte. Für die Herstellung der Elektrodenstruktur werden die Lamellen in das Material geschnitten, welche im Anschluss metallisiert, elektrisch kontaktiert und zur Vermeidung von Korrosion passiviert werden. Die Abdeckplatte, welche ein Fluidzufuhrloch enthält, wird im Anschluss auf die Basisplatte geklebt. Auf die Vorderseite des Aufbaus wird ebenfalls über einen Klebeprozess eine Düsenplatte, aus bspw. einem Kunststoff, aufgebracht. Die enthaltenen Düsen werden über Laserbohrung, mechanisches Bohren, mechanisches Stanzen, Elektronenstrahlbearbeitung oder auch über Ätzprozesse hergestellt (Lee, 2003).

Einen weiteren Tropfenerzeugertyp stellt der Paddle Modus dar (siehe Abb. 1 (b)). Hier werden piezoelektrische Biegefinger (Bimorphaktoren) für die Generierung des Druckimpulses eingesetzt. Piezoelektrische Bimorphanordnungen basieren auf dem Funktionsprinzip von thermischen Bimetallanordnungen (Günther, 2008), bei denen zwei Metalle mit unterschiedlichen thermischen Ausdehnungskoeffizienten verbunden werden. Unter Temperatureinwirkung stellt sich durch die unterschiedliche Dehnung eine Verbiegung der Anordnung ein. Bei piezoelektrischen Biegefingern wird die aktive piezoelektrische Keramik entweder mit einer passiven Membran oder mit einer entgegengesetzt polarisierten piezoelektrischen Keramik

\section{$\square$ Grundkörper $\square$ Piezoelektrischer Aktor $\square$ Fluid}

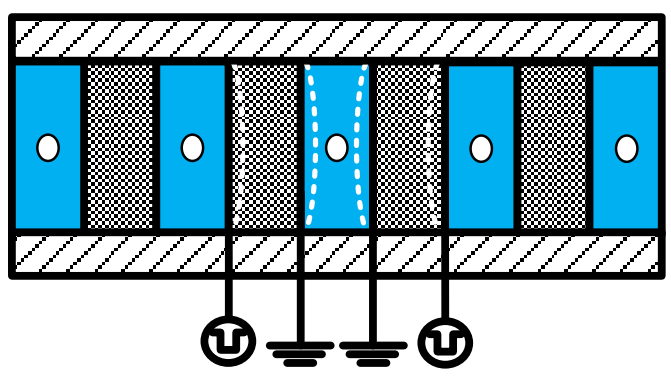

(a)

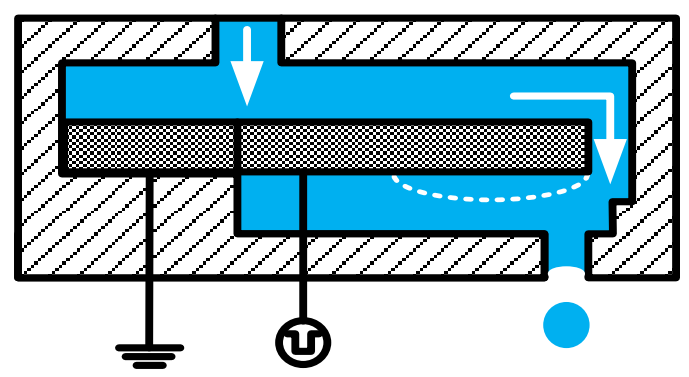

(b)

Abb. 1: Schematische Darstellung eines piezoelektrisch betriebenen Mikrotropfenerzeugers in (a) nach dem Shear Modus mit piezoelektrischer Keramik als Seitenwand (angelehnt an Wijshoff (2010)) und in (b) nach dem Paddle Modus mit piezoelektrischen Biegefinger. 
verbunden. Auch hier erfolgt bei elektrischer Ansteuerung eine Biegung an der Spitze der Anordnung. Hierdurch wird das Fluidkammervolumen unterhalb der Spitze verringert und der Druckimpuls zur Tropfenentstehung generiert. Die Piezokeramik steht direkt in Kontakt mit dem Fluid, womit das Dosierspektrum auf Fluide beschränkt ist, die die verwendete Piezokeramik chemisch nicht angreifen können und zudem elektrisch nicht leitfähig sind. Solch ein Aufbau wird bspw. in Seitz und Heinzl (2004), Ottnad, Irlinger und Lueth (2011) sowie in Ottnad et al. (2012) vorgestellt.

Bei einem piezoelektrisch betriebenen Mikrotropfenerzeuger, der nach dem Squeeze Modus arbeitet, wird ein radial polarisierter piezoelektrischer Aktor um ein fluidgefülltes Röhrchen montiert. Ein elektrischer Impuls führt zur Kontraktion des Aktors. Hierdurch entsteht die benötigte Volumenverringerung in der Kammer und ein Tropfen wird aus der Düse ausgestoßen. In dem ersten kommerziell erhältlichen Tintenstrahldrucker „PT80i“ der Firma Siemens ist dieser Druckkopftyp eingesetzt (Heinzl, 2008). Die Mikrotropfenerzeuger bestehen im Wesentlichen aus einem Röhrchen und einem umschließenden piezoelektrischen Aktor. Als Röhrchenmaterial wird meist auf Glas zurückgegriffen (Lee, 2003). Der Vorteil in der Verwendung eines Glasröhrchens liegt darin, dass zum einen Glas ein chemisch äußerst beständiges Material gegenüber einer Vielzahl von Fluiden ist und zum anderen eine Betrachtung des Tropfenbildungsprozesses von außen möglich ist. Die Röhrchen werden über thermische Prozesse auf ihre geforderten Durchmesser gebracht. An das Ende des Röhrchens kann eine Düse angebracht werden bzw. stellt das Ende des Röhrchens selbst die Düse dar. Abb. 2 (a) zeigt diesen Tropfenerzeugertyp.

Bei einem piezoelektrisch betriebenen Mikrotropfenerzeuger, der nach dem Push Modus arbeitet, drückt ein piezoelektrischer Stab oder oft auch ein Stapelaktor gegen eine Membran (siehe Abb. 2 (b)). Das Anlegen des elektrischen Feldes erfolgt in Polarisationsrichtung der piezoelektrischen Keramik. Die resultierende Biegung ruft auch hier die benötigte Volumenverringerung der fluidgefüllten Kammer hervor. Mikrotropfenerzeuger, die nach dem Push Modus arbeiten, werden mitunter in kommerziellen Drucksystemen der Firmen Epson, Hitachi und Brother eingesetzt (Wijshoff, 2010). Der Vorteil dieses Tropfenerzeugerdesigns ist die Trennung von Fluid und piezoelektrischen Aktor aufgrund der trennenden Membran.

\section{$\square$ Grundkörper $\square$ Piezoelektrischer Aktor $\square$ Fluid}

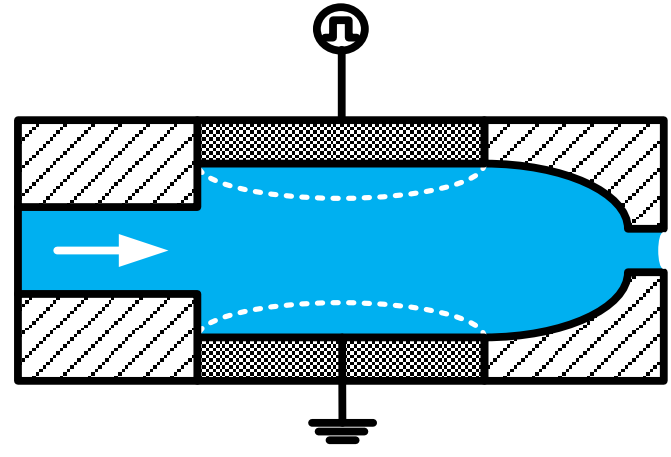

(a)

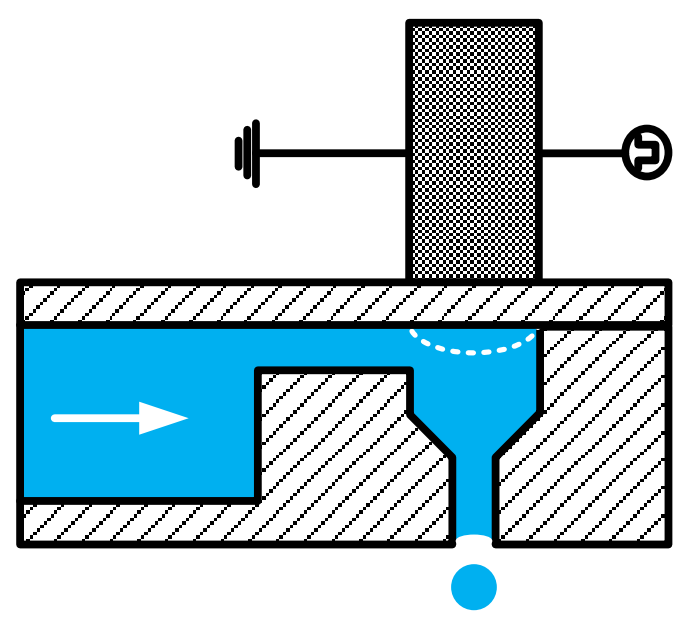

(b)

Abb. 2: Schematische Darstellung eines piezoelektrisch betriebenen Mikrotropfenerzeugers in (a) nach dem Squeeze Modus mit umwickelnden piezoelektrischen Aktor und in (b) nach dem Push Modus mit piezoelektrischen Stabaktor. Angelehnt an Wijshoff (2010). 
Den letzten piezoelektrisch betriebenen Tropfenerzeugertyp stellt der Bend Modus dar. Der Tropfenausstoß wird durch einen einige hundert Mikrometer dicken piezoelektrischen planaren Wandler initiiert, welcher durch eine Membran von der Fluidkammer getrennt ist. Der piezoelektrische Wandler bildet zusammen mit der Membran den Aktor. Auf der zum piezoelektrischen Wandler geneigten Membranseite ist meist eine elektrisch leitfähige Schicht aufgebracht, über die der elektrische Stromkreis des Wandlers geschlossen wird. Das Anlegen eines elektrischen Feldes in Polarisationsrichtung der piezoelektrischen Keramik führt zu der Biegung des Membranaktors in die darunterliegende Fluidkammer. Hierdurch wird ihr Volumen verringert, der notwendige Druckimpuls generiert und als Ergebnis der Tropfen aus der Düse ausgestoßen (siehe Abb. 3). Eine oftmals enthaltene Drossel am Ende der Fluidkammer verhindert in diesem Schritt, dass der Druckimpuls in dahinterliegenden Strukturen, wie bspw. einer Fluidzuführung, entweicht. Die Vorteile dieser Anordnung sind ein äußerst dünner und planarer Aufbau von nur einigen Millimetern Dicke sowie die strikte Trennung von Fluid und der piezoelektrischen Keramik. Somit können auch elektrisch leitfähige Medien dosiert werden. In kommerziellen Druckköpfen wird dieses Design bspw. in Druckern der Firmen Epson, Brother / Kyocera oder Mutoh eingesetzt (Wijshoff, 2010). Solch planare piezoelektrische Aktoraufbauten finden sich aufgrund ihres dünnen und platzsparenden Designs nicht nur in Mikrotropfenerzeugern wieder, sondern auch in anderen mikrofluidischen Produkten, wie bspw. bei Mikroventilen (Scheuenpflug, 2011) oder auch bei Mikropumpen (Herz et al., 2010).

\subsubsection{Einsatz piezoelektrisch betriebener Mikrotropfenerzeuger}

Die Eigenschaft von piezoelektrisch betriebenen Dosiersystemen, Tropfen mit einem Volumen bis hinab in den Pikoliter Bereich zu dosieren und ohne dabei gleichzeitig die chemische Zusammensetzung des Arbeitsfluides zu verändern, ist ausschlaggebend dafür, dass mit konstantem Zuwachs neue Anwendungsgebiete erschlossen werden. Die hervortretenden Applikationen stammen mitunter aus der dreidimensionalen (3D) Drucktechnik, der Medizintechnik, der Elektronikfertigung, der Chemietechnik, der Automobiltechnik, der Fertigung von optischen Systemen oder auch der Pharmazie (Tseng, 2006). Gleichwohl liegt das bislang noch größte Anwendungsfeld für piezoelektrisch betriebene Mikrotropfenerzeuger in der „Home and Office“ Drucktechnik (Elsner, 2009). Exemplarisch werden einige Anwendungsgebiete für piezoelektrisch betriebene Systeme nachfolgend beschrieben.
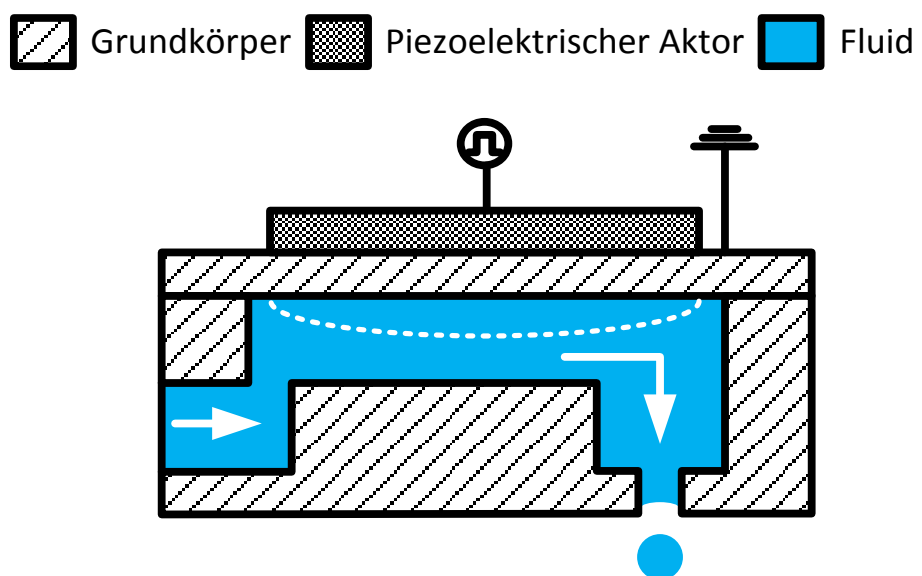

Abb. 3: Schematische Darstellung eines piezoelektrisch betriebenen Mikrotropfenerzeugers nach dem Bend Modus mit Membranaktor. Die piezoelektrische Keramik ist von der Fluidkammer durch eine Membran getrennt. Angelehnt an Wijshoff (2010). 
In dem 3D Druck wird zwischen der Verfestigung eines Pulverbettes mittels Bindemittelauftrag und dem ballistischen Drucken von phasenübergreifenden Medien unterschieden. Die Gemeinsamkeit beider Herangehensweisen ist, dass das Werkstück ebenenweise auf einer verfahrbaren Bauplattform erstellt wird. Für das ballistische Drucken werden bspw. die Medien in einem Fluidreservoir aufgeschmolzen und im flüssigen Zustand dosiert. Nach Auftreffen der Tropfen auf der Bauplattform erfolgt die Rücktransformation des dosierten Stoffes in den festen Zustand. Ottnad, Irlinger und Lueth (2011), Kagerer et al. (2012b) sowie Ottnad et al. (2012) zeigen dies für das 3D Drucken von Wachstropfen. Eine weitere Möglichkeit für das ballistische Drucken ist die Dosierung partikelgefüllter Suspensionen (bspw. Gold, Silber oder Keramiken). Dabei besitzen die enthaltenen Partikel eine Größe von einigen Nanometern bis hin zu wenigen Mikrometern. Entscheidend für die gewählte Partikelgröße ist die Größe der Düse des Tropfenerzeugers. Noguera, Lejeune und Chartier (2005) geben an, dass der Durchmesser der Partikel maximal zwei Prozent des Düsenradius betragen darf, um ein Blockieren der Düse zu verhindern. Nach Auftreffen der Suspension auf dem Substrat sind Postprozesse, wie bspw. Sintern, notwendig. In kommerziellen 3D Drucken im Pulverbett werden Bindemittel tropfenweise in eine Sand- oder auch in eine Gipsschicht dosiert. Diese härtet als Ergebnis aus und der Prozess wird Schicht für Schicht wiederholt.

In der Medizintechnik stehen zum einen strukturierte Biomaterialien, die wiederum spezifische Wechselwirkungen mit Zellen, Proteinen oder auch Desoxyribonukleinsäure eingehen können (Sumerel et al., 2006), und zum anderen die kontrollierte Abgabe von Medien im Pikoliter Bereich im Vordergrund. Hier werden bspw. Mikroarrays aus Zellen zur Medikamentenforschung (Fujita et al., 2013) oder auch Gerüste für die Gewebeabformung (Moore et al., 2006) hergestellt. Piezoelektrisch betriebene Tropfenerzeuger eignen sich besonders als Dosiervorrichtung für diese vergleichsweisen kostenintensiven Medien aufgrund ihrer Eigenschaft der präzisen und kontrollierten Fluidabgabe (Wijshoff, 2010). Ein zusätzliches Merkmal ist, dass nur ein Bruchteil der Energie gegenüber thermisch betriebenen Systemen für den Tropfenausstoß benötigt wird, so dass auch im Umgang mit sensiblen Fluiden keine Gefahr ihrer Beschädigung besteht. In der Medizintechnik gilt es allgemein, lebende Organismen oder komplexe organische Moleküle zu dosieren, die aufgrund ihrer Fragilität keiner großen Beanspruchung ausgesetzt werden dürfen (Lee, 2003).

Ein weiteres Anwendungsgebiet stellt die Elektronikfertigung dar. Die Vorteile gedruckter Elektronikkomponenten sind die mögliche Herstellung von Multilayer Aufbauten, die Verwendung flexibler Basismaterialien, die Elektronikfertigung auf großen Flächen und hierbei gleichzeitig kostengünstiger und effizienter zu arbeiten als die herkömmliche Halbleitertechnik (Parashkov et al., 2005; Wijshoff, 2010). Es werden bspw. elektrische Wiederstände (Sun et al., 2013), Leiterbahnen auf Platinen (Kim et al., 2010), Lötbumps (Lemmermeyer, 2006) oder auch Verbindungsleitungen in Brückenform von Kontaktierungspads (Ko et al., 2010) tropfenweise erstellt. Als Dosiermedien werden partikelversetzte Suspensionen, polymere Dispersionen oder direkt phasenübergreifende Medien, wie bspw. Lote, eingesetzt. In der Fertigung gedruckter Elektronik sind meist Postprozesse, wie bspw. Sintern und Tempern, notwendig.

Es wird an dieser Stelle festgehalten, dass zum einen piezoelektrisch betriebene Tropfenerzeuger in einer Vielzahl von unterschiedlichen Bauformen vorliegen und zum anderen ihr Einsatzspektrum weit über den standardisierten Tintenstrahldruck im „Home and Office“ Bereich hinausreicht. Ihre Eigenschaft, eine Vielzahl von Fluiden mit Tropfenvolumina bis hinab in den Pikoliter Bereich zu dosieren, ohne ihre chemische Zusammensetzung zu verändern, ist für die konstante Generierung neuer Anwendungsfelder verantwortlich (Wijshoff, 2010). 


\subsection{Problembeschreibung / Anwendung - Fertigung von Mikrosystemen}

Aus dem soeben beschriebenen Anwendungsbeispiel der Tropfenerzeugung für die Mikrosystemtechnik wird deutlich, in welcher Vielfalt Mikrosysteme für eine einzige Aufgabe, hier die Tropfenabgabe, vorliegen können. In einem Tropfenerzeuger wirken die drei physikalischen Domänen Mechanik, Elektronik und Fluidik. Die Domäne der Mechanik (bspw. Biegung eines piezoelektrischen Aktors) ist verantwortlich für die Volumenverringerung einer fluidgefüllten Kammer, welche wiederum durch die Domäne der Elektronik (bspw. elektrische Ansteuerung des piezoelektrischen Aktors) hervorgerufen wird. Die Domäne der Fluidik (bspw. Rohrströmung) beeinflusst die Tropfenparameter. Es wird ersichtlich, wie einzelne Komponenten, Strukturen und Materialien in einem Mikrosystem miteinander interagieren, um die gewünschte Funktion hervorzurufen.

Fertigung individueller Mikrosysteme: Hilleringmann (2006) beschreibt die notwendige und individuell auf das Mikrosystem abgestimmte Prozessführung. Während sich Änderungen in der Mikroelektronik, bspw. das Layout von Leiterbahnen als eine zweidimensionale Aufgabe darstellen, ergeben sich bei Mikrosystemen dreidimensionale Aufgaben (Ehrfeld, 2002) mit einer erforderlichen räumlichen Integration der dreidimensionalen Funktionskomponenten (Gerlach und Dötzel, 2006). Die enthaltenen Funktionskomponenten sind meist bewegliche Teile, wie piezoelektrische Aktoren in Mikrotropfenerzeugern oder elektrostatische Kammantriebe in Gyroskopen. Aus diesem Grund bezeichnen Hartzell, da Silva und Shea (2011) die Fertigung eines Mikrosystems auch als eine technische Herausforderung. Hier gilt es, zum einen unterschiedliche dreidimensionale Strukturen und Formen im Mikrometerbereich in einer Vielzahl unterschiedlicher Substrate zu erstellen. Zum anderen werden Funktionswerkstoffe / -komponenten auf den strukturierten Substraten montiert. Die Fügung von dem Funktionswerkstoff zu dem Substrat kann an dieser Stelle über eine Klebstoffschicht erfolgen, welche nach Höhn (2001), Hsu und Clatterbaugh (2004) sowie Dilthey, Brandenburg und Schleser (2005) mit zu den meist eingesetzten Verbindungsverfahren in der Mikrotechnik zählt. Schritt für Schritt werden Bauelemente in hybrider Weise aufeinander montiert, welche wiederum mit unterschiedlichen Fertigungstechniken hergestellt und strukturiert werden. Hybride Architekturen dominieren bislang den Mikrosystementwurf (Hilleringmann, 2006). Hieraus ergibt sich der Sachverhalt, dass auf Mikromontageoperationen in der Fertigung eines Mikrosystems nicht verzichtet werden kann (Fatikow, 2000). Dabei ist die präzise Ausrichtung der Montagepartner zueinander unabdingbar, um eine hohe Leistungsfähigkeit des Mikrosystems zu erzielen (Huff, Bart und Lin, 2011).

Im Ergebnis bedeutet dies, dass sich die Fertigung von Mikrosystemen als eine Kombination aus der Mikrostrukturierung von Substraten und aus der Mikromontage von Bauelementen, wie bspw. Funktionswerkstoffen / -komponenten, darstellt. Im Hinblick auf das Anwendungsbeispiel der Tropfenerzeugung bedeutet dies, bspw. nicht nur die Düsen in ihrer Breite und Länge, sondern auch in ihrer Tiefe zu ändern, um Tropfenparameter über den Düsenquerschnitt zu variieren. Unabhängig von der Mikrostrukturierung der fluidischen Komponenten in dem Substrat gilt es auch, die Mikromontage der einzelnen Bestandteile des Tropfenerzeugers mit gleich hoher Präzision vorzunehmen. Bezogen auf das Anwendungsbeispiel gilt es, die piezoelektrischen Aktoren in einer optimalen Position zu den Düsen des Tropfenerzeugers zu montieren. Am Beispiel eines Tropfenerzeugers, welcher nach dem Paddle Modus arbeitet, tritt die höchste Biegung des Aktors an seinem freien nicht eingespannten Ende auf. Dieses muss sich direkt oberhalb der Düse befinden, so dass der höchste Druckimpuls aufgrund der größten Volumenverringerung direkt an der Düse entsteht. Als Ergebnis steht dem Tropfenbildungsprozess ein erhöhter Energieeintrag zur Verfügung. 
Materialvielfalt: Für Mikrosysteme steht eine umfangreiche Materialauswahl zur Verfügung. Aufgrund seiner guten elektrischen und mechanischen Eigenschaften wird meist Silizium als Substratwerkstoff verwendet. Weitere Substrate sind Gläser, Quarze, Keramiken, Metalle und Kunststoffe (Schwesinger, Dehne und Adler, 2009). Um die eigentliche Funktion im Mikrosystem hervorzurufen, werden Funktionswerkstoffe, wie bspw. Keramiken mit piezoelektrischem Effekt, hinzugefügt. Für die Fertigung eines Mikrosystems werden noch Hilfswerkstoffe, wie bspw. Passivierungs- und Maskierungsschichten, benötigt. Bezogen auf das Anwendungsbeispiel der Tropfenerzeuger bedeutet dies, dass Aktoren aus piezoelektrischen Keramiken, Membranen aus Gläsern und Grundkörper aus Silizium bestehen können. Dabei kann jede Komponente wiederum strukturierte Elemente, wie bspw. Fluidkammern, Drosseln und Düsen im Grundkörper oder Elektroden auf der piezoelektrischen Keramik, enthalten.

Funktionsprüfung: Die konstant steigende Komplexität eines Mikrosystems durch die enthaltene Domänenvereinigung erfordert in frühen Phasen der Entwicklung eine Funktionsprüfung und nimmt in der Mikrosystemtechnik eine immer wichtigere Rolle ein (Ehrfeld, 2002). Im Bereich der Produktinnovationen und -neuentwicklungen müssen daher bereits in frühen Phasen der Produktentstehung die geforderten Eigenschaften des Produktes abgesichert und verifiziert sein. Mit steigendem Produktreifegrad werden Modifikationen an den Bauteilen immer teurer (Zäh, 2006). Diese Kosten können durch den Einsatz von Funktionsmodellen in der Produktentstehungsphase vermieden werden.

Geringe Stückzahlen: Für den Produktentstehungszyklus werden sowohl für Voruntersuchungen als auch für Optimierungsaufgaben Funktionsmodelle in geringen Stückzahlen aus unterschiedlichen Materialien und mit verschiedenen Dimensionen verlangt, die den Aufbau einer eigens geschaffenen Massenfertigungslinie nicht rechtfertigen (Wolf, 2003).

Angepasste Verfahren: Für kleine Stückzahlen werden daher gezielt angepasste Verfahren für die rasche Fertigung von Funktionsmodellen für die Mikrosystemtechnik benötigt (Dauer, Ehlert und Büttgenbach, 1999). Dabei setzt sich die Fertigung der Funktionsmodelle sowohl aus der Mikrostrukturierung von Substraten als auch aus der Mikromontage einzelner Bauelemente, mitunter auch der Funktionswerkstoffe, zusammen. Die Integration dieser Verfahren in den Produktentstehungszyklus ermöglicht, notwendige Änderungen an dem Mikrosystem aufgrund der durchgeführten Funktionsprüfung in kurzer Zeit umzusetzen und hierfür eine Variantenvielfalt mit unterschiedlichen Materialien effektiv und kurzfristig bereitzustellen. So können bspw. an dem Anwendungsbeispiel der Tropfenerzeugung unterschiedliche Düsen-, Drossel-, Fluidkammer- oder auch Aktordimensionen und ihr jeweiliger Einfluss auf die Tropfenentstehung und -parameter untersucht werden. Essentieller Bestandteil bei der Fertigung eines Mikrosystems ist dabei die kontinuierliche Kontrolle des Fertigungsprozesses und v. a. der Prozessergebnisse (Schwesinger, Dehne und Adler, 2009).

Zusammenfassend ist an dieser Stelle festzuhalten, dass für die Funktionsprüfung von Mikrosystemen, welche aus verschiedenen Materialien bestehen, schon in frühen Stadien der Produktentstehung geringe Stückzahlen individueller Funktionsmodelle benötigt werden, die mit einer gezielt angepassten Fertigungstechnik (Mikrostrukturierung von Substraten und Mikromontage von Komponenten) in kurzer Zeit erstellt werden. Somit kann in frühen Phasen der Produktentstehung auf notwendige Änderungen mit einem Redesign des Mikrosystems reagiert werden. Die sich ergebenden messbaren Kriterien sind hierbei: Formtreue des Funktionsmodells, Materialabtragsraten, Anzahl von Prozessschritten und der verwendeten Werkzeuge zur Fertigung eines individuellen Funktionsmodells und die dafür benötigte Zeit, um bspw. rasch das Funktionsmodell bereitzustellen. 


\section{Stand der Technik}

Mikrosysteme lassen sich sowohl in monolithische als auch in hybride Architekturen unterteilen. Während bei einer monolithischen Architektur alle Funktionselemente des Systems, wie bspw. Sensorik, Aktorik oder auch Elektronik, auf einem gemeinsamen Substrat gefertigt werden, bestehen hybride Architekturen aus einzelnen montierten Komponenten. Am Beispiel eines Tropfenerzeugers bedeutet dies, dass in einem Substrat die fluidmechanischen Strukturen, wie bspw. Fluidkammer und Düse, erstellt sind. Um die notwendige Energie für einen Tropfenausstoß zu liefern, wird auf dem strukturierten Substrat eine piezoelektrische Keramik montiert. Der Vorteil hybrider Architekturen ist, dass die einzelnen Komponenten jeweils unabhängig voneinander und mit einer komponentenoptimierten Fertigungstechnik hergestellt werden können. Dieser Sachverhalt ist essentiell dafür verantwortlich, dass hybride Mikrosysteme bislang den Markt dominieren (Hilleringmann, 2006).

Nachfolgend wird der Stand der Technik sowohl für die Mikrostrukturierung, um bspw. fluidmechanische Strukturen in Substraten zu erstellen, als auch für die Mikromontage, um bspw. piezoelektrische Keramiken auf einem Substrat zu montieren, beschrieben.

\subsection{Mikrostrukturierung von Substraten}

Die Mikrosystemtechnik geht aus der Mikroelektronik hervor, so dass im Ergebnis sowohl Werkstoffe als auch Fertigungsverfahren für die Mikrosystemtechnik aus der Mikroelektronik und hierbei insbesondere aus der Complementary metal-oxide-semiconductor (CMOS) Technologie stammen (Ehrfeld, 2002; Lyshevski, 2005; Gerlach und Dötzel, 2006).

Zweifelsohne ist hierbei das Silizium das dominierende Substratmaterial (Hilleringmann, 2006; Globisch, 2011). Dies rührt daher, dass Silizium in großen Mengen verfügbar ist, auf eine bekannte Prozessierbarkeit durch dessen Einsatz in der Mikroelektronik zurückgegriffen werden kann, sowie wegen seiner chemisch reinen Herstellbarkeit und seiner sehr guten elektrischen und mechanischen Eigenschaften. Silizium besitzt eine hohe Bruchfestigkeit und ist elastisch. Darüber hinaus bietet Silizium als Halbleiter den Vorteil der direkten Integration elektronischer, optischer und mechanischer Komponenten auf einem Chip (Schwesinger, Dehne und Adler, 2009). Die Leitfähigkeit von Silizium kann über Dotierprozesse modifiziert werden; über Oxidationsprozesse wird Silizium zu einem elektrischen Isolator.

Für die Herstellung von Mikrosystemen auf Siliziumbasis existiert eine Vielzahl verschiedener Prozesse. Eine lokale Veränderung des Substrates erfolgt mittels physikalischer oder auch chemischer Prozesstechnik (Diffusion und Oxidation). Hiermit werden zum einen die elektrische Leitfähigkeit des Substrates modifiziert und zum anderen werden Isolations- oder auch Maskierungsschichten auf dem Substrat erstellt. Durch chemische und auch physikalische Abscheideprozesse aus der Gasphase oder auch durch Abscheidung aus der flüssigen Phase (bspw. mittels Spin Coating) werden zusätzliche Stoffe auf den Substraten erzeugt. Mikrostrukturtechniken sind notwendig, um miniaturisierte funktionstragende Strukturen für das Mikrosystem im Substrat, wie bspw. Kanäle, Sprungbretter, Membranen etc., zu erstellen (Menz, Mohr und Paul, 2005). Kanalstrukturen werden für mikrofluidische Leitungssysteme oder auch für Düsen in einem Tropfenerzeuger benötigt. Sprungbretter oder auch Membranen dienen zu Detektionen physikalischer Größen in Druck- oder auch Beschleunigungssensoren. 
Für die Erstellung von Mikrostrukturen in Substraten werden meist Nass- und Trockenätzverfahren eingesetzt (Madou, 2002; Jia und Madou, 2006; Schwesinger, Dehne und Adler, 2009). Je nach Dauer und Stärke des Ätzprozesses werden unterschiedlich tiefe Strukturen in dem Substrat erstellt. Um sicherzustellen, dass der Ätzvorgang nur an definierten Stellen auf dem Substrat erfolgt, werden die übrigen Gebiete auf dem Substrat mit einer Maskierungsschicht versehen. Ein wesentlicher Unterschied bei der Strukturierung von Substraten für die Mikrosystemtechnik im Vergleich zu Substraten für die Mikroelektronik ist das erforderliche Aspektverhältnis ( $\triangleq$ Tiefe zu Breite). Während in der Mikroelektronik flache und schmale Strukturen, wie bspw. Leiterbahnen, erstellt werden, besitzen Mikrostrukturen in Mikrosystemen Aspektverhältnisse von bis zu 400 (Jia und Madou, 2006), da sich die Fertigung eines Mikrosystems als eine dreidimensionale Aufgabe im Substrat stellt. Alternative Mikrostrukturierungstechniken, wie bspw. das Funkenerodieren, sind verfügbar. Dennoch werden die bekannten Verfahren des Nass- und Trockenätzens aus der Mikroelektronik übernommen und mit kompatiblen weiteren Prozessen für die Fertigung des Mikrosystems, um bspw. die Fügung von piezoelektrischen Aktoren zu Substraten zu realisieren, ergänzt (Glück, 2005). Grund hierfür ist neben der bekannten und etablierten Prozesstechnik auch die Möglichkeit der Batchprozessierbarkeit (Scheicher, 2004; Gerlach und Dötzel, 2006).

Es werden nachfolgend die bekannten und etablierten maskenbasierten Nass- und Trockenätzverfahren, die Prozessfolge und die notwendige Umgebung bei der Fertigung von Mikrosystemen bzw. bei der Mikrostrukturierung von Substraten beschrieben.

\subsubsection{Prozessablaufplan für die Mikrostrukturierung von Substraten}

Der Prozessablaufplan beschreibt die einzelnen notwendigen Basisschritte, um Mikrostrukturen sowohl mit Nass- als auch mit Trockenätzverfahren in Substraten zu erstellen. Die ersten Schritte hierbei sind die Generierung von Ätzfenstern in einer Maskierungsschicht für das darunterliegende Substrat, an denen die Ätzen Material abtragen. Unabhängig von dem gewählten Ätzprozess werden Maskierungsschichten für die Strukturgebung im Substrat benötigt (Hilleringmann, 2006).

Den Ausgang des vorliegenden Basisprozessablaufplanes bildet ein Siliziumsubstrat (siehe Abb. 4). Hierbei ist dessen Dicke, Dotierung oder auch Kristallorientierung für die weitere Betrachtung nicht von Bedeutung. Voraussetzung für die Mikrostrukturierung von Substraten sind geöffnete Ätzfenster in einer Maskierungsschicht, welche mit der strukturgebenden Photolithographie erstellt werden.

Ideale Maskierungsschichten besitzen eine hohe Selektivität, eine gute Haftung auf dem Substrat und eine porenfreie Schicht. Dabei stellt die Selektivität eine dimensionslose Kennzahl dar, welche die Ätzraten der abzutragenden Schicht und der zu schützenden Maskierungsschicht in das Verhältnis zueinander setzt. Je höher die Selektivität ist, desto länger widersteht die Maskierungsschicht dem Ätzmedium. Je nach Ätzprozess werden unterschiedliche Maskierungsschichten eingesetzt. Es finden Maskierungsschichten aus Silizium, Siliziumdioxid, Siliziumnitrid, Aluminiumoxid, Gold, Wolfram, Titan, Photoresist oder auch Wachse Anwendung (Madou, 2002; Jia und Madou, 2006). Die Wahl des Materials und der Schichtdicke der Maskierung richten sich nach dem verwendeten Ätzverfahren, dem eingesetzten Ätzmedium und der gewünschten Ätzdauer. Das Aufbringen der Maskierungsschichten auf dem Siliziumsubstrat erfolgt mit konventionellen Techniken von thermischer Oxidation über chemischer und physikalischer Gasphasenabscheidung bis hin zu der Abscheidung aus der flüssigen Phase mit bspw. Spin Coating Techniken. 
Nach dem Auftrag der Maskierungsschicht erfolgt deren Strukturierung über Photolithographie, um die Fenster in das darunterliegende Substrat zu öffnen. Hierzu wird eine photoempfindliche Schicht auf der Maskierung aufgetragen. Eines der meist eingesetzten Verfahren hierzu ist das Spin Coating (Globisch, 2011). Die resultierenden Lackdicken variieren von einigen hundert Nanometern für die Mikrotechnik bis hin zu einigen hundert Mikrometern für galvanische Abformungen (Hilleringmann, 2006). Anschließend erfolgt die Belichtung des Photoresistes über eine Maske mit Wellenlängen von unter $400 \mathrm{~nm}$. Die kritische Abmessung bzw. die kleinste abbildbare Linienbreite wird dabei auch von der Wellenlänge der eingesetzten Lichtquelle bestimmt. Hieraus wird das Bestreben der Halbleiterindustrie ersichtlich, die Wellenlänge der Lichtquellen kontinuierlich zu reduzieren. Heutige industriell eingesetzte Lichtquellen stellen Excimerlaser mit einer Wellenlänge von $193 \mathrm{~nm}$ dar. Die verwendete Maske besteht meist aus einem Quarzkern, auf dem die abzubildenden Strukturen in einer Chrom- oder auch Eisenoxidschicht geschrieben sind (Hines et al., 2011). Die Ausrichtung der Maske zu dem Substrat erfolgt dabei in Belichtungsanlagen. Über photolithographische Entwicklungsverfahren werden die übertragenen Strukturen in der Photoresistschicht freigelegt. Mit einem anschließenden Ätzprozess wird die Maskierungsschicht an den offenen Photoresiststellen ebenfalls freigelegt, so dass im Ergebnis das Fenster zu dem darunterliegenden Siliziumsubstrat erstmals geöffnet ist. Auch hier gilt es wiederum, das Ätzmedium, den Ätzprozess und die Ätzdauer optimal auf die beiden Schichten abzustimmen. Den Abschluss bildet der Lift Off des Photoresistes.

Nach der Öffnung des Ätzfensters in der Maskierungsschicht erfolgt der Ätzvorgang für die Mikrostrukturierung des Siliziumsubstrates. Hierbei werden sowohl Nass- als auch Trockenätzverfahren eingesetzt. Um eine exakte Ätztiefe einzuhalten, gibt es neben dem zeitlichen Ätzabbruch bei Kenntnis der Ätzrate auch die Möglichkeit, Ätzstoppschichten hinzuzufügen. Hierzu wird wiederum vorliegender Prozessablaufplan durchlaufen, um an definierten Stellen und an definierter Tiefe im Siliziumsubstrat den Ätzstopp einzubringen. Die Ätzstoppschichten wirken hoch selektiv gegenüber den Ätzmedien. Typische Materialien sind hochdotiertes $\mathrm{p}^{++}$-Silizium oder auch weitere Isolationsschichten.
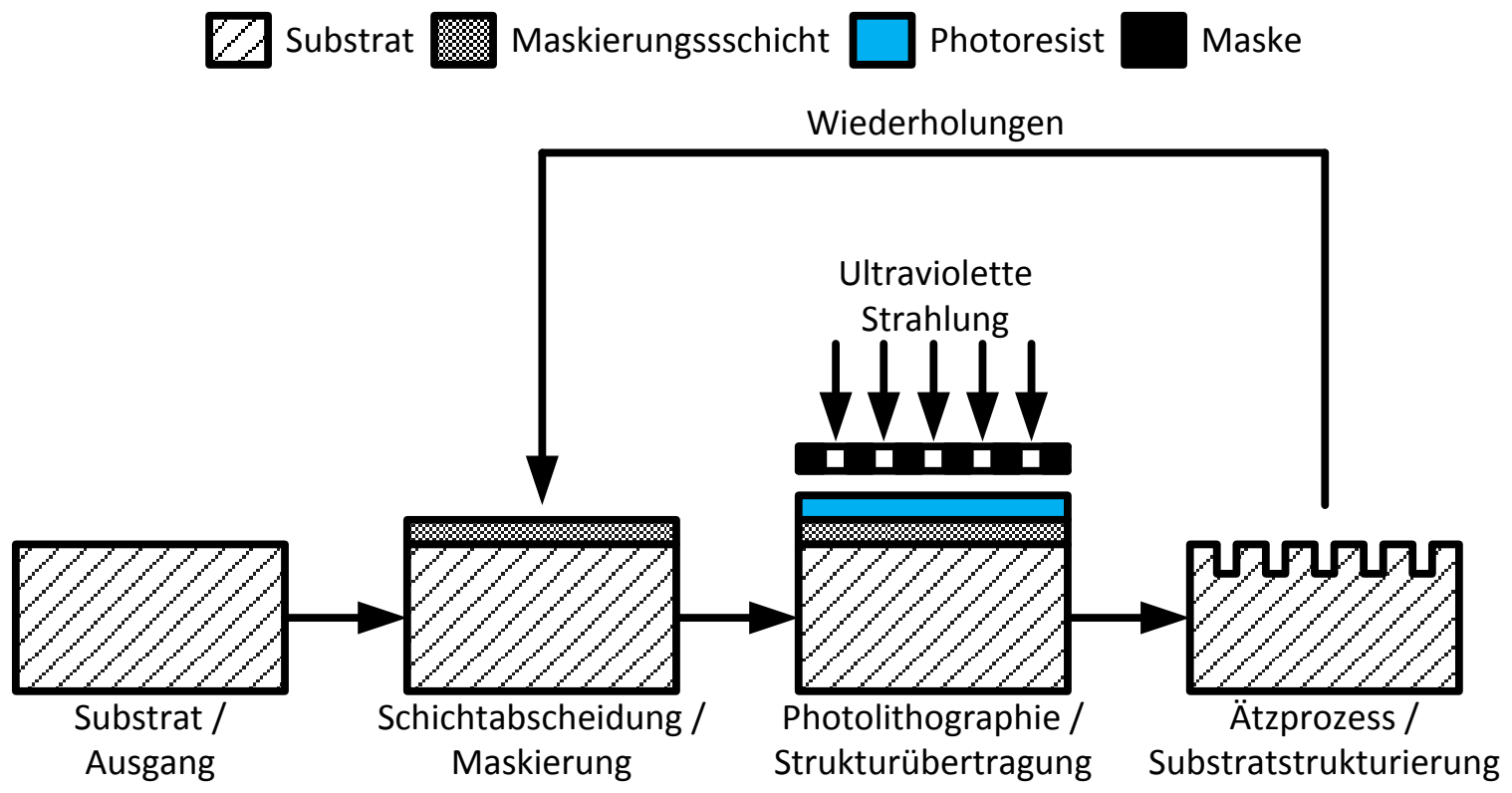

Abb. 4: Prozessfolge bei der Mikrostrukturierung von Siliziumsubstraten mit konventionellen maskenbasierten Nass- und Trockenätzverfahren. Neben der Maskierung der prozessrelevanten Substratseite gilt es auch, die Seitenwände und die Rückseite zu maskieren, um das Ätzen an diesen Stellen zu verhindern (nicht gekennzeichnet). 
Nicht inkludiert in o. g. Prozessablaufplan sind Nebenprozesse, wie bspw. die Reinigung von Substraten, die Temperung von Photoresist, der Schutz der nicht prozessaktiven Substratseite gegen das Ätzmedium oder auch die ständig geforderte Qualitätskontrolle. Der Prozessablaufplan für die Mikrostrukturierung von Siliziumsubstraten für deren Einsatz in Mikrosystemen ist durch eine wiederholende Prozessschrittfolge, die gezielte Anpassung der einzelnen Prozesse auf die Gegebenheiten der Materialkombinationen und die meist beidseitige Prozessierung der Siliziumsubstrate gekennzeichnet. Werden bspw. unterschiedlich tiefe Strukturen im Substrat benötigt, erfolgt für jede einzelne Strukturtiefe ein Ätzvorgang mit vorausgegangener Maskierung bzw. Passivierung, um bereits erstellte Strukturen nicht weiter zu ätzen. Hierbei muss der Ätzprozess gezielt an vorhandene Strukturen angepasst werden (Wolf, 2003). Bei Mikrosystemen werden meist doppelseitige Strukturierungsprozesse gefahren. Am Beispiel eines Mikrotropfenerzeugers, welcher nach dem Bend Modus arbeitet (vgl. Abb. 3), bedeutet dies, dass über der Substratoberseite die Fluidkammern oder auch Drosseln und über der Substratrückseite die Düsen im Substrat erstellt werden. Hierfür wird zweimal o. g. Prozessablaufplan inkl. Rückseitenschutz und Maskenlayout vollzogen.

\subsubsection{Basisprozesse für die Mikrostrukturierung von Substraten}

In der Mikrotechnik werden Ätzverfahren sowohl für das Übertragen einer photolithographisch erzeugten Struktur in das Substrat als auch zum ganzflächigen Abtrag von Schichten eingesetzt. Ein ganzflächiger Abtrag ist bspw. für die Entfernung von nativem Siliziumdioxid notwendig, welches nicht stöchiometrisch rein und für nachfolgende Prozesse störend ist.

Die Ätzverfahren werden einerseits nach ihren Techniken (nass oder trocken) und andererseits nach ihren ausübenden Ätzprofilen unterschieden. Grundsätzlich wird zwischen einem isotropen ( $\hat{=}$ richtungsunabhängigen) und einem anisotropen ( $\hat{=}$ richtungsabhängigen) Ätzprofil unterschieden (siehe Abb. 5). Mit (2.1) wird der Grad der Anisotropie Ani bestimmt. Entspricht die laterale Ätzrate $\mathrm{R}_{\text {Lat }}$ der vertikalen Ätzrate $\mathrm{R}_{\mathrm{Ver}}$, entsteht ein isotropes Ätzprofil einschließlich der Unterätzung der Maskierung (siehe Abb. 5 (a)).

$$
A n i=1-\frac{R_{\text {Lat }}}{R_{\text {Ver }}}
$$

Wird dagegen nur in die Tiefe geätzt, entsteht ein ideales anisotropes Ätzprofil mit einem Anisotropiegrad von eins (siehe Abb. 5 (c)). Folglich wird das Maskierungsfenster direkt in das darunterliegende Substrat übertragen. Gründe für eine isotrope bzw. anisotrope Ätzprofilausbildung stellen materialspezifische energetische Wechselwirkungen mit Ätzmedien oder auch im Allgemeinen Ätzprozessparameter dar.

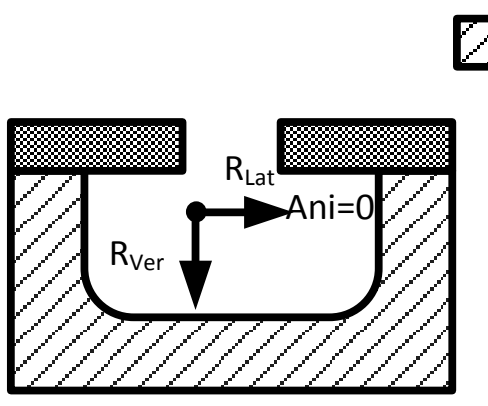

(a)

Substrat Maskierungsschicht

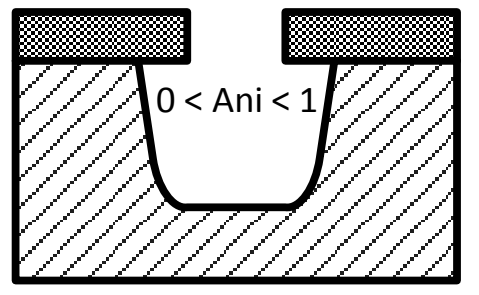

(b)

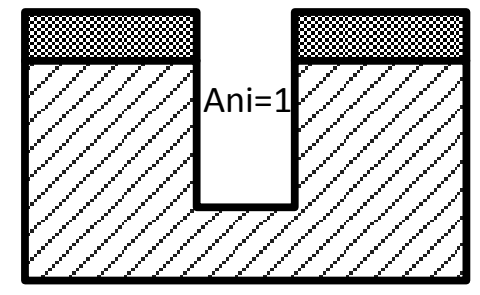

(c)

Abb. 5: Schematische Darstellung des Anisotropiegrades. (a) Ideales isotropes, (b) teilweise anisotropes und (c) ideales anisotropes Ätzprofil. Angelehnt an Tadigadapa und Lärmer (2011). 


\subsubsection{Nassätzverfahren für die Mikrostrukturierung von Substraten}

Mit dem nasschemischen Ätzen wird das feste Substrat in eine flüssige Verbindung überführt. Hierbei finden sowohl saure als auch basische Ätzlösungen Anwendung. Über die Konzentration und die Temperatur werden sowohl Ätzrate als auch Oberflächenrauheit der geätzten Gebiete beeinflusst. Moleküle der Ätzlösung reagieren mit Oberflächenatomen des Substrates, welche hierdurch aus dem Feststoffverbund gelöst werden (Schwesinger, Dehne und Adler, 2009). Die nasschemischen Ätztechniken formen in der Regel ein isotropes Ätzprofil (Hilleringmann, 2006; Globisch, 2011). Allerdings gibt es auch hier Ausnahmen. Silizium besitzt aufgrund seiner Kristallographie Vorzugsrichtungen für basische Ätzlösungen, so dass anisotrope Ätzprofile erstellt werden können.

Für das nasschemische Ätzen gibt es zwei Techniken: die Tauch- und die Sprühätzung. Während bei der Tauchätzung Substrate in ein Ätzbecken eingetaucht werden, befinden sich die Substrate bei der Sprühätzung auf einer Schleudertrommel und werden mit der Ätzlösung besprüht. Wichtiger Vorteil der Sprühätzung ist die konstante Ätzmittelkonzentration über den gesamten Prozesszeitraum aufgrund der kontinuierlichen Zuführung reinen Ätzmittels. Dem gegenüber steht jedoch der Vorteil der Batchprozessierbarkeit von Tauchätzungen.

Saures nasschemisches Ätzen von Silizium erfolgt unter dem Einsatz von Gemischen aus Fluss- (HF) und Salpetersäure $\left(\mathrm{HNO}_{3}\right)$, welche mit Pufferzusätzen versehen sind. Die Pufferzusätze lösen dabei Silizium-Fluor-Verbindungen. Silizium reagiert mit Salpetersäure zu Siliziumdioxid, welches durch die enthaltene Flusssäure in der Ätzlösung wiederum abgetragen wird. Die Ätzlösung ist stark genug, um kristallographische Gegebenheiten von Silizium nicht beachten zu müssen. Im Ergebnis entsteht ein isotropes Ätzprofil. Die Nachteile des sauren chemischen Nassätzens von Silizium liegen in der Verbreiterung der Strukturen durch die resultierende Maskierungsunterätzung und der meist geringen Selektivität gegenüber der Maskierungsschicht selbst. Auf der anderen Seite bietet es den Vorteil von hohen Ätzraten bis über $800 \mu \mathrm{m} / \mathrm{min}$, welche allerdings erst nach erfolgreicher Beseitigung der genannten Nachteile industriell einsetzbar sind (Schwesinger, Dehne und Adler, 2009).

Mikroelektronische Integrationstechniken erfordern in der Regel keine Ätzung in das Substrat. Lediglich für Grabenisolationen oder auch Trench Kapazitäten erfolgt eine einige Mikrometer tiefe Ätzung in das Siliziumsubstrat. Im Gegensatz dazu benötigen Mikrosysteme für die Herstellung ihrer dreidimensionalen Komponenten eine anisotrope Ätzung des Siliziumsubstrates (Hilleringmann, 2006). Beispiele hierfür sind Mikrokanäle in fluidmechanischen Produkten oder auch Membranen und Biegebalken in Sensoren. Hierfür eignet sich vor allem das anisotrope nasschemische Ätzen von Siliziumsubstraten mit Basen. Als Orientierung für das richtungsgebundene Ätzen dient die Kristallographie des Siliziums. Silizium besteht aus zwei kubisch-flächenzentrierten Gittern. Jedes Gitter besteht aus Würfeln, bei denen sich an jeder Ecke wiederum ein Atom und sechs weitere Atome in der Mitte jeder Würfelfläche befinden. Die Anordnung der einzelnen Atome führt dazu, dass sich unter dem Einsatz spezieller Ätzlösungen in jeder Raumrichtung unterschiedlich hohe Ätzraten ergeben. Dies führt zu der Anisotropie des basischen Nassätzens. Besonders die $\{111\}$ Ebenen im Siliziumkristall stellen ätzbegrenzende Ebenen dar, da jedes Atom hier Bindungen zu drei Nachbaratomen aufweist, die senkrecht zur Ätzrichtung liegen. Hieraus ergibt sich ein höherer Energiebedarf zum Herauslösen eines Atoms im Vergleich zu den anderen Ebenen. Der Vorteil, dass sich $\{111\}$ Ebenen als ätzbegrenzend ergeben, wird zum Entwurf von unterschiedlichen Strukturen verwendet. Meist werden Alkalilaugen, wie bspw. Kaliumhydroxid (KOH), Natriumhydroxid $(\mathrm{NaOH})$ oder auch Lithiumhydroxid $(\mathrm{LiOH})$, eingesetzt. 
Abb. 6 zeigt die auftretende Anisotropie bei dem basischen nasschemischen Ätzen von Siliziumsubstraten. Je nach Ausrichtung der Kristallebenen im Siliziumsubstrat werden unterschiedliche Ätzergebnisse erzielt. Ist das Substrat $\langle 100\rangle$ orientiert, entsteht in Abhängigkeit der Ätzdauer das bekannte pyramidenförmige Ätzprofil unter einem Winkel von 54,74 (siehe Abb. 6 (a)). Ist das Substrat $<110\rangle$ orientiert, stehen die ätzbegrenzenden $\{111\}$ Ebenen hierzu unter einem Winkel von $90^{\circ}$ und die Strukturen weisen senkrechte Seitenwände auf (siehe Abb. 6 (b)). Beim anisotropen nasschemischen Ätzen gilt es, besonders auf Hillocks ( $\hat{=}$ sporadische Materialanhäufungen) und auch auf die Unterätzung konvexer Ecken zu achten.

\subsubsection{Trockenätzverfahren für die Mikrostrukturierung von Substraten}

Trockenätzverfahren in der Mikrostrukturierung ermöglichen reproduzierbare und homogene Ätzungen für nahezu sämtliche Materialien in der Mikrosystemtechnik (Hilleringmann, 2006). Hierbei werden drei verschiedene Ätzmechanismen unterschieden: Rein chemischer Mechanismus (bspw. Plasmaätzen), rein physikalischer Mechanismus (bspw. Ionenstrahlätzen) und eine Kombination aus beiden Mechanismen (bspw. reaktives Ionenätzen).

Die wichtigsten Trockenätzverfahren sind hierbei das Ionenstrahlätzen, Plasmaätzen und reaktive Ionenätzen (Glück, 2005). Das Ionenstrahlätzen wird hierbei meist nur in der Forschung und Entwicklung eingesetzt (Schwesinger, Dehne und Adler, 2009), da dieses sowohl eine geringe Ätzrate als auch Selektivität aufweist. Weitaus wichtiger sind die Plasmaätzverfahren. Hierbei wird zwischen dem reinen Plasmaätzen und dem reaktiven Ionenätzen unterschieden. Reaktives Ionenätzen stellt dabei das wichtigste Trockenätzverfahren dar (Glück, 2005; Zorman und Mehregany, 2006; Schwesinger, Dehne und Adler, 2009).

Der Materialabtrag erfolgt bei dem Plasmaätzen durch chemische Reaktionen. Hieraus resultiert ein isotropes Ätzprofil. Mikrosysteme benötigen funktionstragende Strukturen im Substrat, welche allerdings nur durch ein anisotropes Verhalten erstellt werden können. Hieraus ergibt sich der Sachverhalt, dass das Plasmaätzen meist für das Abtragen und die Reinigung ganzer Oberflächenschichten auf Substraten eingesetzt wird. Ein Beispiel hierfür ist das Veraschen von Photoresist. In einer Reaktionskammer wird über das Anlegen eines hochfrequenten Wechselfeldes (HF) eine Gasentladung gezündet. Dies resultiert in einem ätzaktiven

27 Siliziumsubstrat $\cong$ Maskierungsschicht $\square$ Abgetragenes Silizium

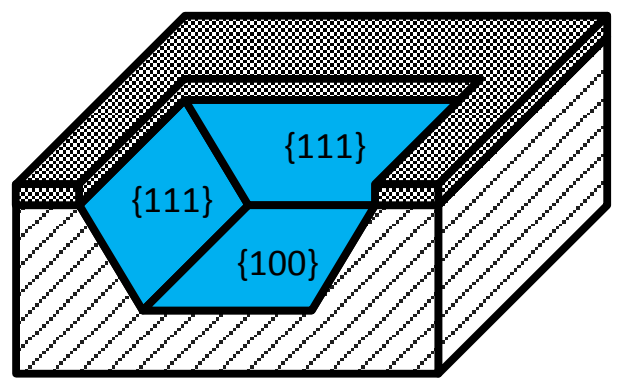

(a)

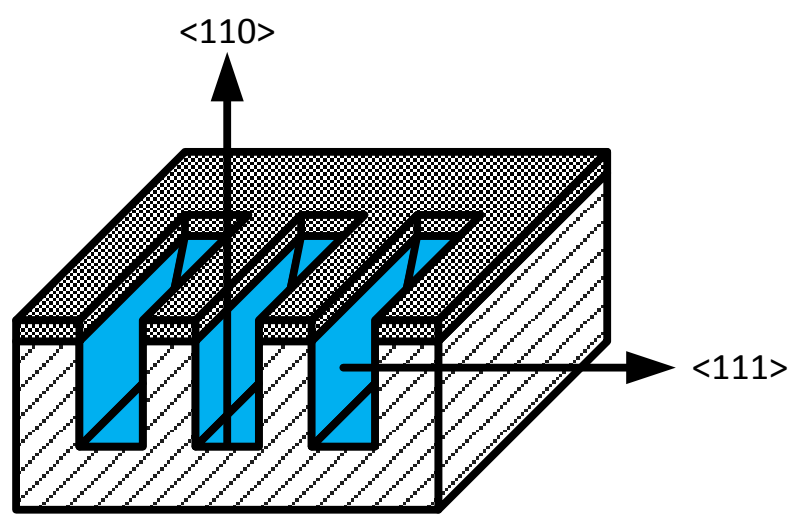

(b)

Abb. 6: Schematische Darstellung des anisotropen nasschemischen Ätzens von Silizium mit basischer Ätzlösung. (a) Silizium mit <100> und (b) Silizium mit <110> Orientierung. Unterätzung der Maskierungsschicht nicht gekennzeichnet. Angelehnt an Globisch (2011). 
Plasma. Aufgrund der Stöße im Plasma dissoziiert das eingelassene Gas. Neben Ionen entstehen auch ätzaktive Radikale, welche dem Substrat zugeführt werden. Sofern die Radikale mit den Oberflächenatomen flüchtige Verbindungen eingehen können, kommt es zum Materialabtrag. Für die Ätzung von Silizium werden fluorhaltige Ätzgase, wie bspw. Tetrafluormethan $\left(\mathrm{CF}_{4}\right)$ oder auch Schwefelhexafluorid $\left(\mathrm{SF}_{6}\right)$, eingesetzt. Über eine Vakuumpumpe werden die Reaktionsprodukte aus der Reaktionskammer abgesaugt. Das maskierte Substrat befindet sich auf der geerdeten Elektrode. Die entstehenden Ionen werden zur negativ geladenen Elektrode beschleunigt, so dass auf das Substrat nur die ätzaktiven Radikale geleitet werden.

Anisotrope Ätzprofile können mit trockener Ätzung durch das Verfahren des reaktiven Ionenätzens erstellt werden. Das Siliziumsubstrat liegt auf der mit hochfrequenter Wechselspannung beaufschlagten Elektrode. Charakteristisch für das reaktive Ionenätzen ist, dass eine Kombination sowohl aus chemischen als auch physikalischen Ätzmechanismen Anwendung findet. Die im Plasma erzeugten Ionen werden zu der HF Elektrode beschleunigt und rufen auf dem Substrat einen physikalischen anisotropen Materialabtrag hervor. Die HF Elektrode ist im Vergleich zu der Gegenelektrode sehr klein, so dass diese ein negatives Potential (Biasspannung) annimmt. Die zusätzlich entstandenen ätzaktiven Radikale rufen den chemischen Ätzmechanismus hervor. Durch das Aufschlagen der Ionen auf dem Substrat ist dessen Substratoberfläche geschädigt, so dass die chemische Reaktion begünstigt wird. Über die Einstellung der Biasspannung wird der Ätzprozess gesteuert. Hohe Biasspannungen rufen stärker beschleunigte Ionen hervor. Im Ergebnis bedeutet dies, dass sich ein anisotropes Ätzprofil ausbildet, jedoch sinken hierdurch auch Selektivitäten und die Gefahr von Strahlenschäden im Substrat steigt. Die Höhe der Biasspannung ist ein Kompromiss zwischen geforderter Anisotropie und Selektivität. Weitere Einflussgröße ist der Druck in der Prozesskammer. Je höher der Druck ist, desto dominanter ist der chemische Ätzmechanismus, da die Ionen weniger stark beschleunigt werden. Für das reaktive Ionenätzen von Silizium werden meist die gleichen Ätzgase wie bei dem Plasmaätzen in Verbindung mit Trägergasen eingesetzt. Typische Strukturierungstiefen mit dem reaktiven Ionenätzen liegen bei ca. $20 \mu \mathrm{m}$ (Schwesinger, Dehne und Adler, 2009). Dies reicht aber für die Mikrostrukturierung von Siliziumsubstraten für Mikrosysteme oftmals nicht aus. Um dieser Forderung nachzukommen, wurde das Verfahren des tiefen reaktiven Ionenätzens (DRIE) entwickelt. Dieses stellt eine alternierende Kombination von Ätz- und Beschichtungsprozessen dar. In der gleichen Prozesskammer wird abwechselnd sowohl das Ätzgas als auch das Prozessgas zur Bildung polymerer Passivierungsschichten an den Oberflächen und Seitenwänden zugegeben. Die Passivierungsschicht legt sich um die Strukturen. Im Anschluss wird erneut das Ätzen vollzogen, so dass die Passivierungsschichten an den Oberflächen abgetragen werden. Die Seitenwände sind nach wie vor passiviert und bieten weiteren Schutz vor den ätzaktiven Radikalen. Im Ergebnis werden hiermit tiefe und schmale anisotrope Strukturen erstellt.

\subsubsection{Reinheit in der Mikrostrukturierung von Substraten}

Da die Basisprozesse der Mikrosystemtechnik denen der Mikroelektronik entsprechen, gelten auch bei der Fertigung von Mikrosystemen die gleichen Anforderungen an die Fertigungsumgebung wie bei der Mikroelektronik. Wie bereits dargestellt, ist die Fertigung und hierbei insbesondere die Mikrostrukturierung von Substraten ein äußerst technisch komplexer Gesamtprozess, welcher wiederum aus vielen Einzelschritten, wie bspw. Photolithographie, Schichtabscheidung und Schichtabtragung, besteht. Jeder Fehler in einem Einzelschritt kann im Ergebnis zu dem Ausfall des gesamten Systems führen. Besonders kritisch ist hierbei der Photolithographieschritt, da hier enthaltene Fehler direkt in das Substrat über die Maskierungsschicht übertragen werden. Von besonderer Bedeutung sind sowohl die Oberflächenqua- 
lität (bspw. Ebenheit, Rauheit oder Parallelität) der Siliziumsubstrate / Wafer als auch die Vermeidung von Partikeln und chemischen Verunreinigungen (Gerlach und Dötzel, 2006). Die Oberflächenqualität der Substrate wird durch deren Hersteller garantiert, so dass während der Fertigung die Vermeidung von Partikeln und chemischen Verunreinigungen im Vordergrund steht. Die Verunreinigung von Partikeln wird durch den Einsatz von Reinräumen minimiert, dessen Luft, je nach Reinraumklasse, eine minimale Anzahl Partikel bestimmter Größe enthält. Chemische Verunreinigungen werden durch Reinigungsprozesse minimiert.

Reinräume gewährleisten saubere Umgebungsbedingungen. Ihre Klassifizierung kann nach DIN EN ISO 14644-1 (Stand Juli 1999) erfolgen. Diese unterteilt die einzelnen Klassen nach der Anzahl von Partikeln bestimmter Größe je Kubikmeter Luft. Um die Kosten für den Betrieb des Reinraumes zu reduzieren, wird dieser in den Weißbereich (geringe Partikelanzahl für die Substratbearbeitung) und in den Graubereich (höhere Partikelanzahl für den Servicebereich) unterteilt. Die Verteilung der einzelnen Stationen im Reinraum erfolgt wiederum in die Bereiche Dünnschichttechnik (Beschichtung, Photolithographie und Nasschemie), Analytik und Plasmabehandlung (Völklein und Zetterer, 2006). Dabei stellt die Photolithographie mit der geringsten Partikelanzahl, einer stabilen Temperaturverteilung und konstanter Luftfeuchte die höchsten Anforderungen.

Die fehlerarme Fertigung von Mikrosystemen erfordert neben einer minimalen Partikelanzahl auch eine Minimierung der chemischen Verunreinigungen. Chemische Verunreinigungen werden nach ihrer Zusammensetzung unterschieden. Hierbei gibt es organische, anorganische und metallische Verunreinigungen. Diese entstehen bspw. durch unzureichenden Lift Off von Photoresist, Abrieb von Produktionsanlagen oder auch durch Absputtern von Metallteilchen. Generell führen die Verunreinigungen zu einer schlechten Schichthaftung, zu Substratfehlern oder auch zu Strukturfehlern. Um dies zu vermeiden, besteht die Fertigung von Mikrosystemen nicht nur aus den Basisprozessen, sondern auch aus einer Vielzahl von Nebenprozessen, wie bspw. Reinigungsvorgängen. Dabei sind die wichtigsten Reinigungsprozesse der Einsatz von Lösungsmitteln und auch Trockenreinigungsprozesse. Vor allem der nach der Firma Radio Corporation of America (RCA) benannte nasse Reinigungsprozess gilt als Standardreinigungsverfahren für die Entfernung von organischen und metallischen Verunreinigungen.

Als Zwischenfazit lässt sich feststellen, dass die Fertigung von Mikrosystemen den gleichen strengen Bedingungen wie die Fertigung von mikroelektronischen Produkten unterliegt. Dies bedeutet im Detail, dass sowohl der Einsatz eines Reinraumes als auch ständige Reinigungsprozesse wichtige Bestandteile des Fertigungsprozesses sind. 


\subsection{Mikromontage in der Mikrosystemtechnik}

Das Ausschöpfen des eigentlichen Potentials der Funktionsintegration und der Miniaturisierung in der Mikrosystemtechnik ist erst durch die Kombination einzelner Komponenten zu einem hybriden Mikrosystem möglich (Dilthey und Brandenburg, 2005; Schilp, 2012). Diese Mikrosysteme können aus Komponenten bestehen, die mit jeweils angepasster Fertigungstechnik und geeigneter Materialauswahl hergestellt worden sind. Auch piezoelektrisch betriebene Tropfenerzeuger zählen zu der Gruppe hybrider Mikrosysteme, da die Fügung des piezoelektrischen Wandlers auf einer Basis über einer Klebschicht einen Montageschritt verlangt (Hilleringmann, 2006). Weitere bekannte hybride Architekturen sind nach Schilp (2012) sowohl biologische MEMS als auch Micro-Opto-Electro-Mechanical-Systems. Produkte hieraus sind bspw. Lab-on-a-Chip Systeme oder auch Mikrospiegel für Laserprojektionsdisplays.

Hybride Architekturen dominieren den Markt der Mikrosysteme (Hilleringmann, 2006). Monolithische Architekturen auf einem Chip gelangen oftmals an prozesstechnische und wirtschaftliche Grenzen, um alle Funktionen auf einem Chip und ohne Montageprozesse zu vereinen. DIN 32564-1 (Stand August 2012) definiert ein hybrides Mikrosystem als ein ...

„.... Mikrosystem, bei dem die Integration von Mikrokomponenten und Funktionseinheiten mittels spezieller Mikromontage-, Mikrojustage-, Aufbau- und Verbindungstechniken erfolgt."

Das Montieren ist definiert als die Summe aller notwendigen Vorgänge, die für den Zusammenbau von geometrisch bestimmten Körpern notwendig sind. Daraus leitet sich ab, dass Montagen im Kern aus den Vorgängen des Fügens nach DIN 8593-0 (Stand September 2003) und aus Möglichkeiten der Werkstückhandhabung nach VDI 2860 (Stand August 2012) bestehen (Lotter, 2012). Zusätzlich werden noch Tätigkeiten sowohl für das Justieren und Kontrollieren als auch für Sonderoperationen, wie bspw. Markieren oder auch Erwärmen, benötigt.

Eine allgemein genutzte Definition des Begriffes der „Mikromontage“ existiert bislang noch nicht (Raatz et al., 2012). In Verbindung mit der Definition von Begriffen zu Fertigungsmitteln für Mikrosysteme erfolgt in der DIN 32564-2 (Stand Mai 2004) erstmals eine Definition des Begriffes Mikromontage als der ...

„... Zusammenbau mikrotechnischer Bauteile, Aufbau von Mikrokomponenten auf Montageflächen oder deren Einbau in Gehäuse einschließlich elektrischer Kontaktierung und Erstellung sonstiger Anschlüsse. “

Dementsprechend bleibt festzuhalten, dass die Mikromontage in der Mikrosystemtechnik zum einen für den kompletten Zusammenbau hybrider Mikrosysteme aus Einzelkomponenten und zum anderen meist für die Gehäusung und die elektrische Kontaktierung monolithischer Mikrosysteme eingesetzt wird (Petersen, 2003). Der Mikromontageprozess setzt sich dabei aus den beiden Kernvorgängen des Handhabens und des Fügens zusammen.

\subsubsection{Mikromontage im Vergleich zu standardisierten Montagetechniken}

Nachfolgend wird die Abgrenzung der Mikromontage zu konventionellen Montagetechniken wie der Montage in der Makrowelt, der Oberflächenmontage in der Elektronikproduktion und der Aufbau- und Verbindungstechnik in der Mikrosystemtechnik beschrieben. 
Die Abgrenzung der Mikromontage gegenüber den Montagetechniken aus der Makrowelt erfolgt sowohl über die Bauteilgröße als auch über die notwendigen Montagegenauigkeiten. Greitmann (1998) stellt hierzu erstmals eine Einteilung in fünf Montagekategorien vor (siehe Abb. 7). Raatz et al. (2012) beschreibt, dass der Begriff Mikromontage als Synonym für die Mikropräzisionsmontage, Präzisionsmontage und auch Mikromontage nach Abb. 7 verwendet wird. Dies bedeutet, dass selbst für makroskopische Bauteile mit Abmessungen größer fünf Millimeter, Montagegenauigkeiten kleiner 25 Mikrometer erreicht werden müssen.

Pick \& Place Anlagen sind bereits seit Langem in der Fertigung von Elektronikprodukten im Einsatz. Dabei hat sich die Surface Mount Technology (SMT) etabliert. Es werden Bauteile mit genormten und gleichbleibenden Abmaßen, sog. Surface Mount Devices (SMD), auf Schaltungsträgern montiert. Durch den hohen Standardisierungsgrad und den begrenzten Fügetechniken ist eine Vielzahl von Montageautomaten erhältlich, die über 60.000 Baueinheiten pro Stunde verarbeiten. Die Montagegenauigkeiten liegen dabei zwischen feinwerktechnischer Montage und Mikromontage (Raatz et al., 2012). Allerdings besteht zwischen der Montage in der Mikroelektronik zu der in der Mikrosystemtechnik kaum Ähnlichkeit. Pick \& Place Anlagen verfügen über eine ungenügende Flexibilität in der Montage von Mikrosystemen. Mikrosysteme benötigen die Dreidimensionalität, unterschiedliche Fügeprozesse, ein hohes Formen- und Materialspektrum und vor allem Möglichkeiten zu der Montage von nicht standardisierten Komponenten (Fatikow, 2000).

Monolithische Mikrosysteme werden auf Basis eines Substrates gefertigt. Für ihre Inbetriebnahme werden noch Aufbau- und Verbindungstechniken benötigt. Zu der Aufbautechnik gehören Verfahren der Gehäusung und des Verschlusses (bspw. anodisches Bonden) des Mikrosystems. Zur Verbindungstechnik zählen Basisprozesse, wie bspw. das Löten oder auch das Kleben, die wiederum Teil der Mikrofügetechniken sind. Prozesse der Aufbau- und Verbindungstechnik verlaufen meist parallel. Dem gegenüber stehen die seriellen Prozesse aus der Mikromontage, wie bspw. das Greifen oder das Positionieren. Die inhaltlichen Überschneidungen zwischen der Mikromontage und der Aufbau- und Verbindungstechnik verlaufen jedoch fließend (Nienhaus, 1999; Schilp, 2012).

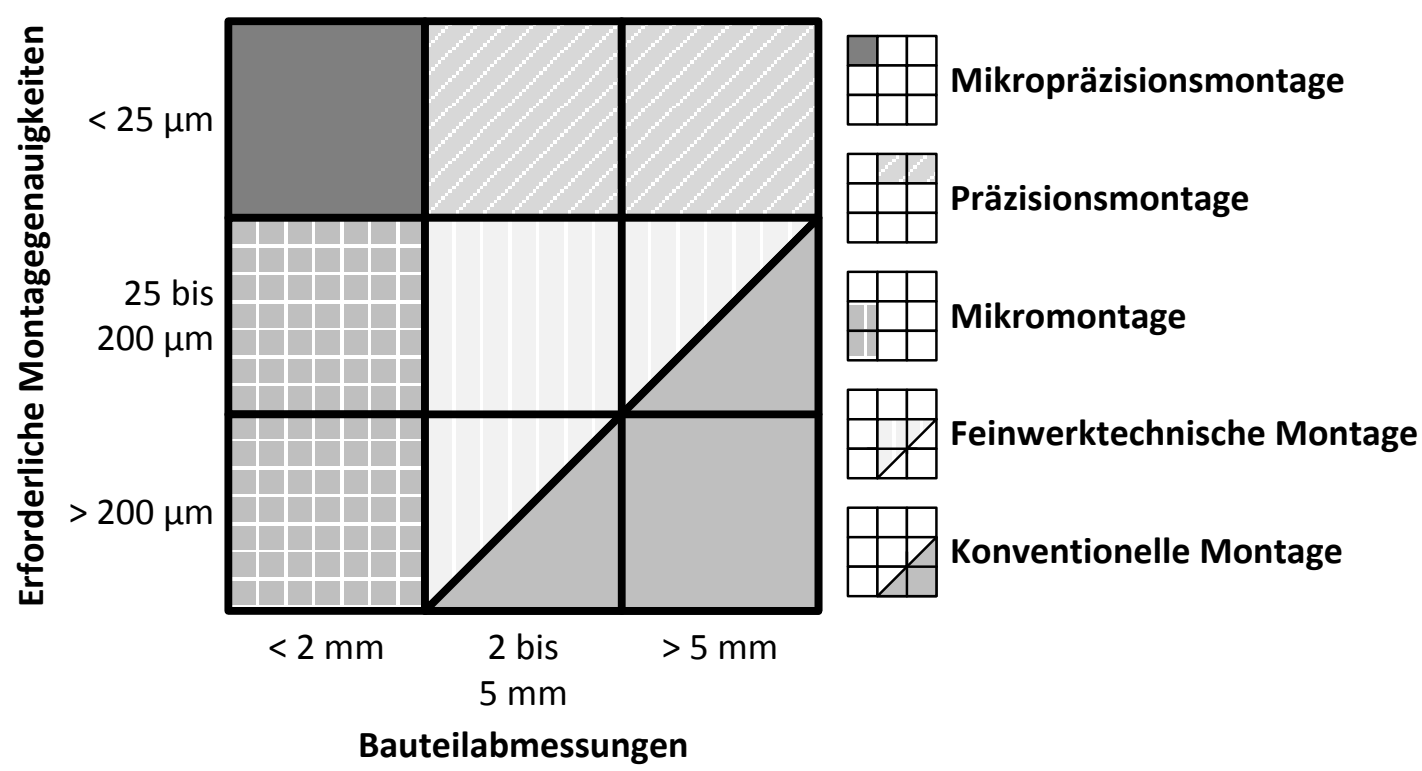

Abb. 7: Klassifizierung der Montagetechniken in Bezug auf die Bauteilabmessungen und auf die Montagegenauigkeiten. Angelehnt an Greitmann (1998) und Raatz et al. (2012). 


\subsubsection{Prozessablauf in der Mikromontage}

Der Begriff der Mikromontage wird vorliegend als Kombination von Handhabungs- und Fügeoperationen verstanden. Abb. 8 verdeutlicht diese Definition an dem notwendigen Prozessablauf bei der Mikromontage.

Der Mikromontageprozess wird in drei Basisprozesse unterteilt. Nach einer geordneten, orientierten und vereinzelten Zuführung des Werkstückes erfolgt der Greifvorgang. Der Greifer wird oberhalb des Werkstückes positioniert. Im Anschluss wird das Bauteil gegriffen und aufgenommen. Der Greifvorgang kann mit sensorischen Funktionen unterstützt werden. Nach dem Greifvorgang wird das Werkstiuck zu dem Montageort transferiert. Es erfolgt erneut ein Positionierschritt, um das Werkstück an einer definierten Position auf dem Montageobjekt abzusetzen. Dies kann ebenfalls sensorunterstützt erfolgen, um das Werkstück präzise zu fügen. Vor oder auch nach dem eigentlichen Fügeprozess wird die Verbindung von Werkstück zu Montageobjekt durch Verbindungstechniken, wie bspw. Klebe- oder Lötprozesse, realisiert. Im Anschluss wird der Greifer aus dem Arbeitsraum herausgeführt.

Nachfolgend werden unterschiedliche Methoden bzw. Gerätschaften für die Mikromontage vorgestellt.

\subsubsection{Gerätschaften für die Mikromontage}

Gerätschaften für die Mikromontage werden anhand ihres Automatisierungsgrades nach manuelle, telepräsente und automatisierte Systeme unterschieden. Nachfolgend werden sowohl die Mikromontagemöglichkeiten als auch Verfahren für den Klebstoffauftrag in Mikromontageanlagen aufgezeigt. Die Fokussierung auf den Klebstoffauftrag begründet sich dadurch, dass dieser zu den meist eingesetzten Fügeverfahren in der Mikrosystemtechnik zählt (Höhn, 2001; Hsu und Clatterbaugh, 2004; Dilthey, Brandenburg und Schleser, 2005) und vor allem Anwendung bei piezoelektrischen Aktoren findet.

$\square$ Arbeitsplattform / Montageort $\cong$ Greifer $\square$ Werkstück
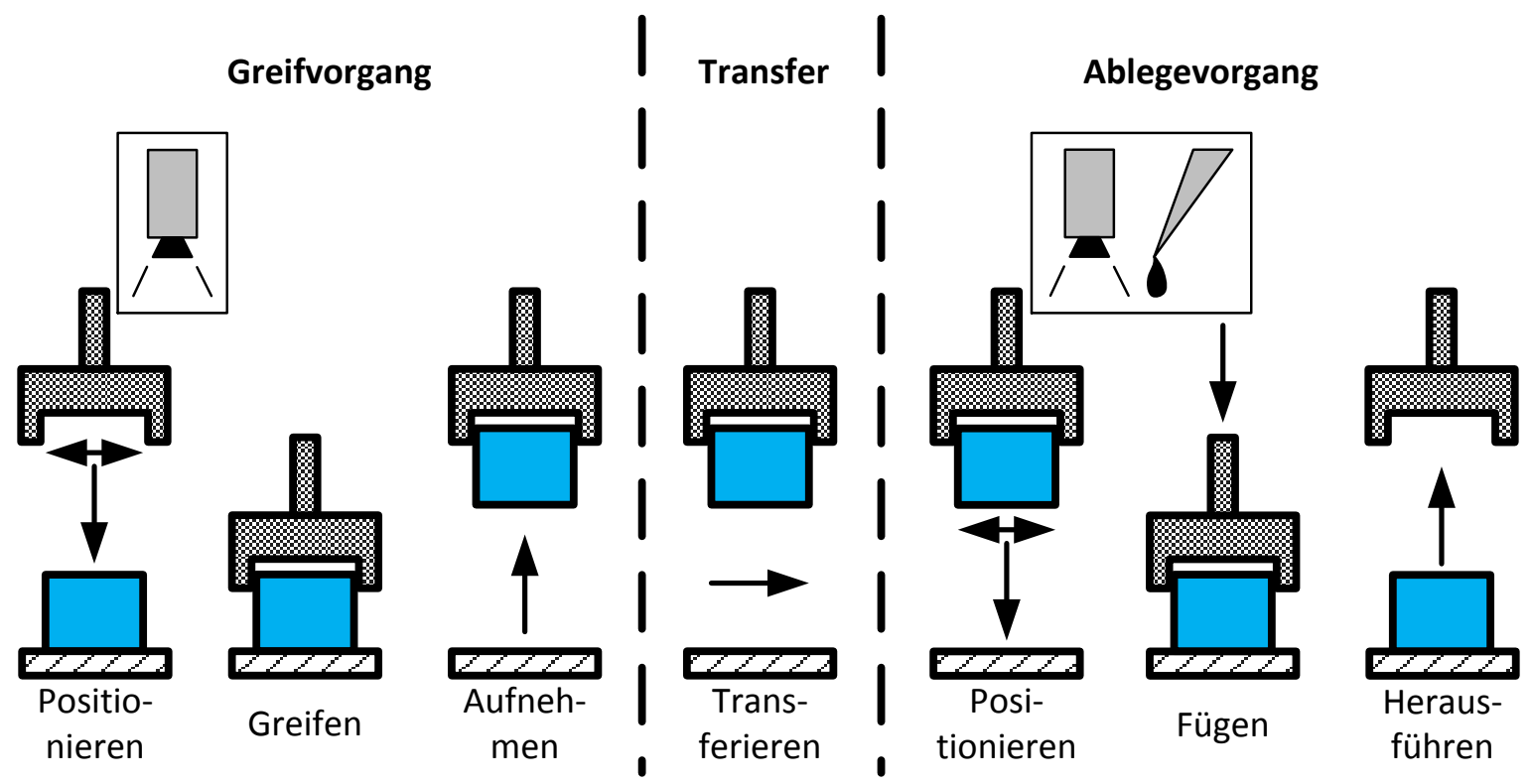

Abb. 8: Prozessablauf in der Mikromontage bestehend aus dem Greifvorgang, dem Transfer und dem Ablegevorgang. Angelehnt an Seckner (2008). 


\subsubsection{Manuelle Mikromontageanlagen}

Bei manuellen Mikromontageanlagen führt der Operator alle relevanten Arbeitsvorgänge, wie bspw. das Montieren, das Greifen, das Justieren, das Positionieren und das Fügen, mit manuellen Bewegungen an den Werkstücken oder auch an den Betriebsmitteln aus. Dabei können ihm Hilfsmittel wie Seh- oder Handhabungsmittel zur Verfügung stehen. Das zu bestückende Bauteil wird auf einer Fixierplattform befestigt. Mit Hilfe einer Aufnahmevorrichtung, wie bspw. einem Vakuumgreifer, wird das zu platzierende Bauteil aufgenommen. Beide Montagepartner werden zueinander ausgerichtet. Im Anschluss erfolgt der Absetzvorgang.

Schilp (2012) beschreibt, dass manuelle Mikromontageanlagen, wie bspw. der Firmen arteos und MARTIN, im Wesentlichen aus folgenden Komponenten bestehen: Systeme zur Grobund Feinjustierung der Montagepartner zueinander, Werkzeuge zum Manipulieren, Greifen und Fügen der Bauteile (wie bspw. Vakuum- und Zweibackengreifer, Dispens- oder auch Lötwerkzeuge), Beobachtungseinheiten für den Montageprozess (wie bspw. Mikroskope oder auch Kameras) und Peripherie (wie bspw. Netzteile oder auch Gestelle). Die Ausrichtung der Montagepartner zueinander erfolgt mit Hilfe von Justagemarkierungen und Kontaktmustern auf den Bauteilen. Ein Beispiel hierzu ist die Bestückung von Platinen mit SMD Bauteilen. Die Abstandsmaße der Kontaktpads auf den Platinen entsprechen denen der SMD Bauteile, so dass einzig die Bauteile zueinander ausgerichtet werden müssen. Relativpositionieraufgaben werden nicht durchgeführt.

Manuelle Mikromontageanlagen stellen einen Großteil der Montagesysteme dar, da viele Mikromontagen von Mikrosystemen in Klein- und Mittelserien mit Stückzahlen von unter 100 pro Tag erfolgen (Petzold, 2008; Raatz et al., 2012). Die Systeme zeichnen sich durch ihre geringe Umrüstzeit bzgl. neuer Aufgaben und Randbedingungen aus (Schilp, 2012).

So können beispielsweise an der manuellen Mikromontageanlage TOMM 1 der Firma arteos Vakuumgreifer mit Zweibackengreifer ausgetauscht oder mit Dispensköpfen umgerüstet werden (siehe Abb. 9 (a)). Mit diesem System können Bauteile zwischen $1 \mu \mathrm{m}$ und $10 \mathrm{~mm}$ Bauteildimension gegriffen und montiert werden. Ein weiteres manuelles System ist der Reworkplatz EXPERT 04.6 der Firma MARTIN (siehe Abb. 9 (b)). Dieser ist hauptsächlich für die manuelle Bestückung von Platinen mit SMD Bauteilen vorgesehen.

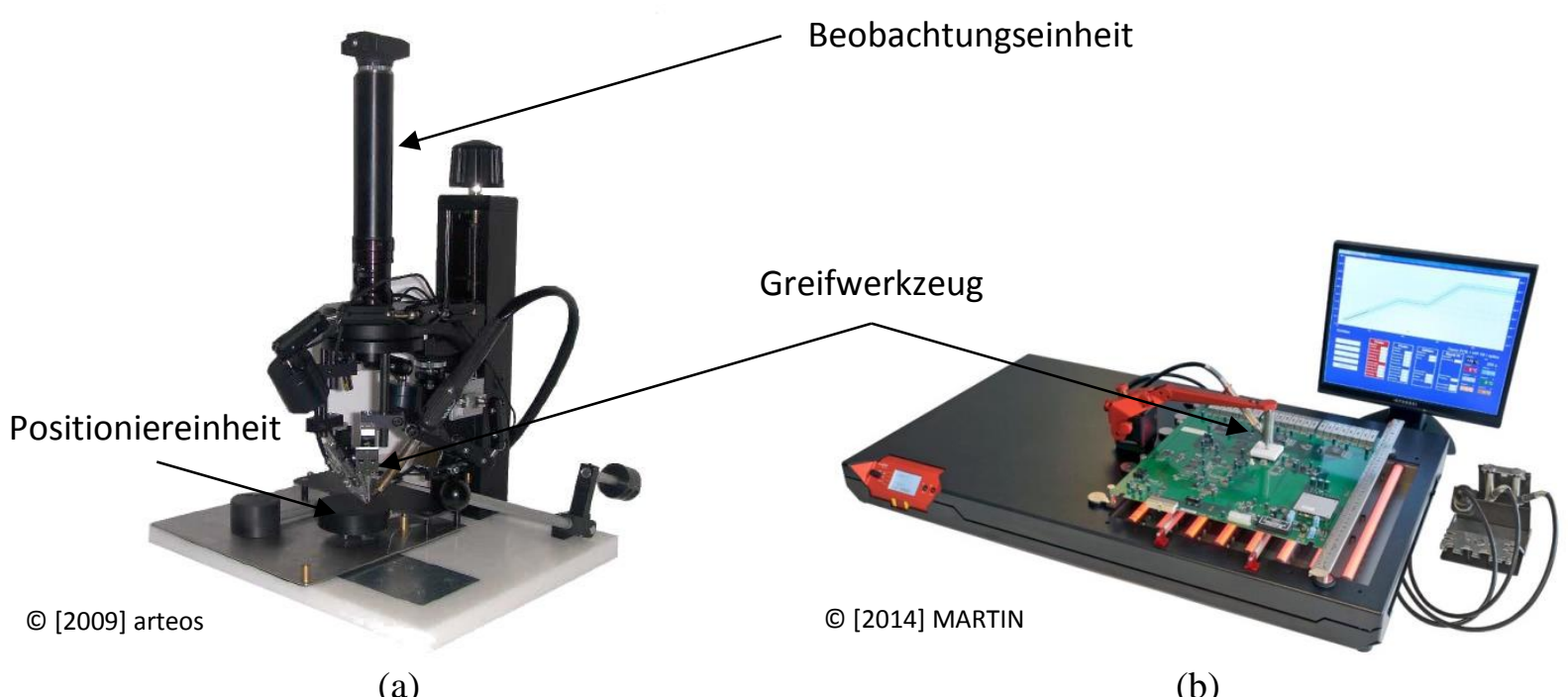

(a)

(b)

Abb. 9: Manuelle Mikromontageanlagen (a) TOMM 1 der Firma arteos und (b) EXPERT 04.6 der Firma MARTIN. (a) entnommen aus arteos (2009) und (b) aus MARTIN (2014). 


\subsubsection{Teleoperierte Mikromontageanlagen}

Die Weiterentwicklung von manuellen Mikromontageanlagen stellen teleoperierte Systeme dar. Hiermit wird die Prozessstabilität erhöht, da manuelle Bewegungen am Werkstïck oder auch an den Betriebsmitteln durch den Operator vermieden werden.

Bei der teleoperierten Mikromontage werden die Bewegungen des Operators über Eingabegeräte, wie bspw. Joysticks, direkt an den Teleoperatoren, wie bspw. Präzisionsrobotern mit Greifern, im Arbeitsraum der Montagestationen umgesetzt. Beobachtungssysteme im Arbeitsraum übertragen die Bewegungen an Monitore. Fatikow (2000) beschreibt, dass die meisten teleoperierten Mikromontageanlagen aus fünf Komponenten bestehen: Die Manipulationseinheit, das Operationsmodul, die Beobachtungseinheit, die Steuerung und Module zur Kraftrückkopplung. Denn nur durch eine realitätsnahe Kraftrückkopplung der Endeffektoren werden Beschädigungen bei dem Absetzvorgang der fragilen Bauteile vermieden. Dabei kann eine akustische oder haptische Meldung ausgegeben werden. Die größten Unterschiede von telepräsenten Mikromontageanlagen liegen bei den Antriebsarten für die Manipulation von Objekten und bei der visuellen Rückkopplung (Fatikow, 2000). Mögliche Aktuationsprinzipien basieren bspw. auf der Elektrostatik, der Piezoelektrizität, der Hydraulik oder auch auf dem Formgedächtniseffekt. Für die visuelle Rückkopplungen werden bspw. standardisierte Kameras, Virtual Reality oder auch Rasterelektronenmikroskope eingesetzt.

Das teleoperierte Mikromontagesystem der Firma Klocke Nanotechnik als Beispiel in Abb. 10 verfügt über piezoelektrisch betriebene Manipulatoren mit Wiederholgenauigkeiten im Nanometerbereich. Das dargestellte System ist mit einem X / Y-Kreuztisch mit einem Bearbeitungsfeld von 50 x $50 \mathrm{~mm}^{2}$ und einer verfahrbaren Höhenachse ausgestattet. Die Steuerung der Manipulatoren erfolgt über kraftrückgekoppelte Joysticks. Je nach Konfiguration kann das System sowohl telepräsent als auch automatisiert betrieben werden.

Schilp (2012) beschreibt, dass im Gegensatz zu rein manuellen Mikromontageanlagen der Aufwand der Umrüstung oder auch der Erweiterung bzgl. neuer Aufgaben durch die notwendige Anbindung neuer Werkzeuge an die proprietären Steuerungsarchitekturen steigt. Die Stückzahlenflexibilität ist gegenüber rein manuellen Mikromontageanlagen gleichbleibend, allerdings erhöht sich die Prozessstabilität aufgrund der eingesetzten Teleoperatoren.

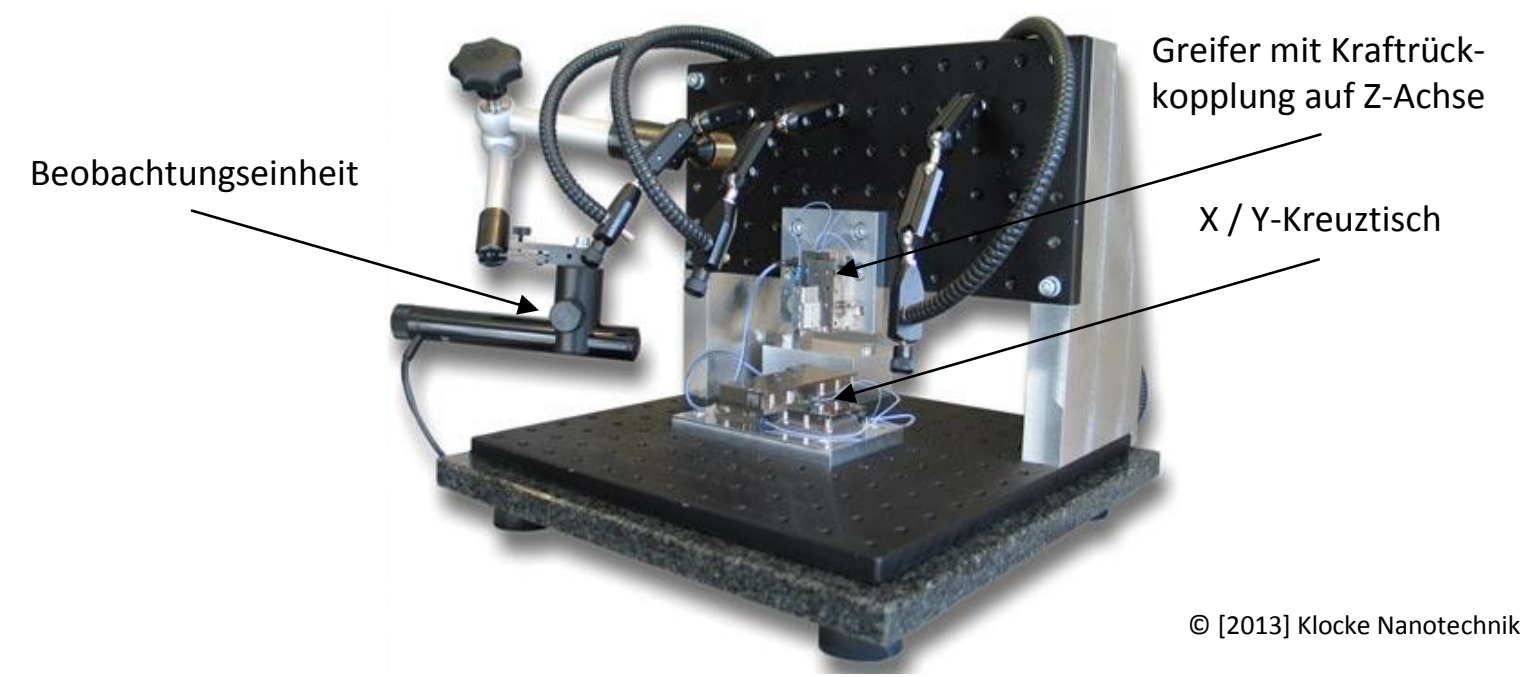

Abb. 10: Teleoperierte Mikromontageanlage MPS (Grundversion) der Firma Klocke Nanotechnik. Entnommen aus Klocke Nanotechnik (2013). 


\subsubsection{Automatisierte Mikromontageanlagen}

Höhere Stückzahlen hybrider Mikrosysteme werden mit automatisierten Mikromontageanlagen gefertigt. Es erfolgt eine schrittweise Bearbeitung der Montageaufgabe, während Zuführund Verkettungseinrichtungen neue Bauteile zuführen bzw. weiterreichen (Seckner, 2008).

Zeilinger (2013) beschreibt, dass automatisierte Mikromontageanlagen im Wesentlichen aus sechs Funktionseinheiten bestehen: Der Steuerungstechnik, den Endeffektoren, den Sensoren zur Lagebestimmung, der Zuführungstechnik, den Kinematik- und Positioniersystemen und auch aus den prozessspezifischen Zusatzmodulen. Die Kommunikation der einzelnen Einheiten erfolgt meist über ein Bussystem. Kernelement jeder automatisierten Mikromontageanlage ist die Steuerungstechnik für die Koordination der Funktionseinheiten. Kinematik- und Positioniersysteme beeinflussen die erzielbaren Montagegenauigkeiten und die umsetzbaren Fügeorientierungen. Mögliche Aktorsysteme sind Linearantriebe, piezoelektrische Antriebseinheiten oder auch SCARA (Selective Compliance Assembly Robot Arm) Kinematiken mit Schrittmotoren (Fatikow, 2000). Die Schnittstelle zwischen Kinematik und Bauteil bilden die Endeffektoren, wie bspw. die Greifer. Sensoren, wie bspw. Kameras, Lichttaster oder inkrementelle Messtaster, werden zur Bestimmung der Bauteillage oder auch für die Zustandsüberwachung der Greifer verwendet. Zusatzmodule werden für Sonderaufgaben, wie bspw. Werkstückwendung, eingesetzt. Die letzte Einheit ist die Zuführungstechnik. Hiermit werden die zu montierenden Komponenten den Anlagen zugeführt. Das Magazinieren der Komponenten kann bspw. in Blistergurten, Wafflepacks oder auch in Formnestern erfolgen. Die Bauteile werden über Förderbänder oder spezielle Halter für das Magazinieren bereitgestellt.

Abb. 11 zeigt als Beispiel die automatisierte Mikromontageanlage VICO Base der Firma Häcker Automation. Das Maschinendesign ist modular aufgebaut. In einer möglichen Beispielskonfiguration (VICO 520 M) ist die Anlage neben Greifern noch mit einem Dispenssystem ausgestattet. Die Positioniergenauigkeit beträgt $\pm 10 \mu \mathrm{m}$ und die Dosiergenauigkeit $\pm 0,1$ nl. Die Systeme können mit unterschiedlichen Werkzeugen ausgestattet werden.

Im Vordergrund aller automatisierten Mikromontageanlagen stehen sowohl die Prozessstabilität und -flexibilität als auch die Produkt- und Variantenflexibilität. Nachteilig wirken sich allerdings die niedrige Erweiterungs- und Änderungsflexibilität aus, da Änderungen im Anlagenaufbau nur mit hohem technischem Aufwand realisiert werden können (Schilp, 2012).

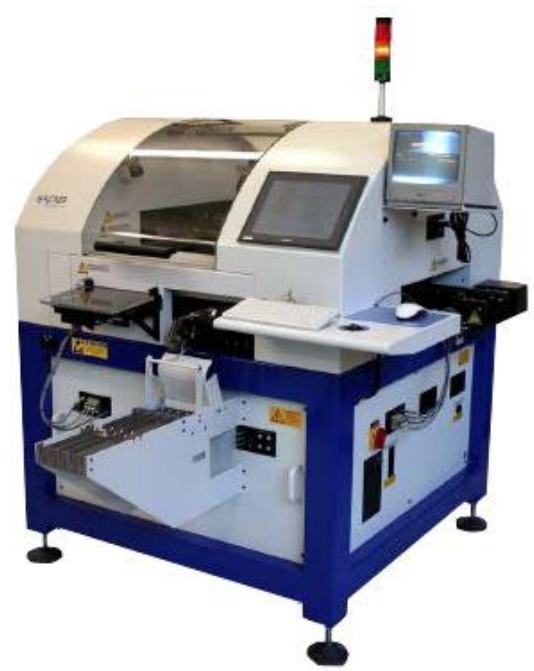

(C) [2014] Häcker Automation

Abb. 11: Automatisierte Mikromontageanlage VICO Base der Firma Häcker Automation. Entnommen aus Häcker Automation (2014). 


\subsubsection{Klebstoffauftragsverfahren in Mikromontageanlagen}

Das Kleben zählt zu den wichtigsten stoffschlüssigen Fügetechniken von Mikrokomponenten und gewinnt zunehmend an Bedeutung (Höhn, 2001; Hsu und Clatterbaugh, 2004; Dilthey, Brandenburg und Schleser, 2005). Gegenüber anderen Fügetechniken, wie bspw. Bonden, Löten oder auch Schweißen, ergeben sich Vorteile wie eine geringe Wärmebelastung der Bauteile, einer großflächigen Anwendung, einer hohen Prozessflexibilität bzgl. Fügepartnerwerkstoffen oder auch einem Gewichtsvorteil gegenüber mechanischen Verbindungsverfahren (Dilthey, Brandenburg und Schleser, 2005; Bobzin et al., 2011; Hesse, 2012). Dies erklärt auch die Tatsache, dass die größte Gruppe von prozessrelevanten Endeffektoren Dispenssysteme in Mikromontageanlagen darstellen (Zeilinger, 2013). Auf dem Markt befindet sich eine Vielzahl an Klebstoffen, die der Stoffgruppe der Polymere zugeordnet werden. Es wird zwischen Polyadditions-, Polymerisations- und Polykondensationsklebstoffen unterschieden (Schwesinger, Dehne und Adler, 2009). Durch Zugabe von Additiven und Füllstoffen können die Eigenschaften des Klebstoffes modifiziert werden, so dass bspw. elektrisch leitfähige oder chemisch äußerst beständige Klebstoffe entstehen. Klebstoffe werden in der Mikrosystemtechnik für das Montagekleben (bspw. piezoelektrischer Wandler auf Membran), das Leitkleben (bspw. Ersatz von Lötprozessen), das Fixierkleben (grobe Fixierung für nachfolgenden Lötprozess), das Schutzkleben (Schutzschicht fragiler Komponenten) oder auch das Ausfüllen von Unebenheiten eingesetzt. Je nach Anwendung werden Klebstoffvolumina bis hinab in den Pikoliterbereich benötigt. Die einzelnen Verfahren können zum einen hinsichtlich ihrer Auftragsform (ganzflächig oder punkt- / linienförmig) und zum anderen hinsichtlich ihres Abstandes zum Substrat (berührend oder berührungslos) unterschieden werden. Typische Klebschichtdicken liegen in der Mikrosystemtechnik zwischen 1 und $20 \mu \mathrm{m}$ (Dilthey, Brandenburg und Schleser, 2005).

Eines der wichtigsten berührenden Klebstoffauftragswerkzeuge sind Dispenser mit Kanülen, meist Dosiernadeln (siehe Abb. 12 (a)). Nahezu jeder Mikromontageanlagenbauer bietet diese Dosierwerkzeuge als Zubehör in seiner Produktpalette an. In industriellen Anwendungen werden aus den Nadeln keine Einzeltropfen ausgestoßen. Es wird ein flacher Meniskus an der Nadelöffnung erzeugt. Bei Kontakt des Meniskus mit dem Substrat bildet sich eine Klebstoffsäule aufgrund der Oberflächenspannung aus, die beim Hochfahren der Nadel abreißt und eine reproduzierbare Menge an Klebstoff auf dem Substrat hinterlässt. Die Verfahren werden in Druck-Zeit und Volumen Dispenser eingeteilt (Höhn, 2001; Habenicht, 2009). Bei volumengesteuerten Dispensern wird meist über einen Schritt- oder Gleichstrommotor ein Kolben im Reservoir bewegt, so dass im Ergebnis Klebstoff verdrängt wird. Aufgrund des präzisen einstellbaren Weges und eines meist langsamen Vorschubes erfolgt eine sehr präzise Dosierung. Ein weiteres volumengesteuertes Dispenssystem sind Schneckenantriebe, die mit einer archimedischen Schraube den Vorschub erzeugen. In Druck-Zeit Dispenssystemen wird über einen zeitlich begrenzten Druckstoß der Klebstoff ausgestoßen. Mit beiden Verfahren werden Tropfenvolumina bis hinab in den Pikoliterbereich ausgestoßen (Schilp, 2001). Es werden punkt- und linienförmige Muster erzeugt. Ein weiteres wichtiges Auftragsverfahren ist der Siebdruck (siehe Abb. 12 (b)). Dieser wird oft in der SMD Montage eingesetzt (Höhn, 2001; Dilthey, Brandenburg und Schleser, 2005; Habenicht, 2009). Dabei wird der Klebstoff durch ein Sieb, welches Aussparungen in Form der Auftragsgeometrie enthält, gerakelt. Die Unterseite des Siebes ist zum Teil beschichtet, so dass ein Fadenziehen des Klebstoffes bei der Rückfederung des Siebes vermieden wird (Dilthey, Brandenburg und Schleser, 2005). Auf ähnlichem Prinzip basiert auch der Schablonendruck. Anstatt eines Siebes wird eine Schablone aus Metallblech verwendet. Durch den Einsatz von Metallblechen werden dickere Klebschichten erzielt, die sich nach der Dicke des Siebes / Schablone richten. Ein weiteres berüh- 
rendes Klebstoffauftragsverfahren ist die Pin-Transfer-Technik mit Stempelwerkzeugen (siehe Abb. 12 (c)). Hierbei wird ein Stempel in eine dünne und definierte Klebstoffschicht eingetaucht. Im Anschluss wird der adhäsiv haftende Klebstoff mit einer Stempelbewegung auf das Substrat übertragen. Dilthey, Brandenburg und Schleser (2005) beschreiben, dass sich die Stempeltechnik durch einen preiswerten und einfachen Aufbau auszeichnet und dass vor allem ein großer Viskositätsbereich von Klebstoffen eingesetzt werden kann. Nachteilig wirken sich die adhäsiven und rheologischen Eigenschaften des Klebstoffes und des Stempels auf die Präzision aus, so dass der Prozess mehr Schwankungen als bspw. bei Dispenssystemen unterliegt (Höhn, 2001).

Ein berührungsloses Klebstoffauftragsverfahren stellt die Tropfenerzeugung mit Druckköpfen dar (siehe Abb. 12 (d)). Hierzu werden meist Piezo Jet Verfahren eingesetzt. Mit Tropfenerzeugern können minimale Klebstoffvolumina bis hinab von $30 \mathrm{pl}$ ausgestoßen werden (Dilthey, Brandenburg und Schleser, 2005). Größere Volumina werden bspw. über höhere Aktuationsfrequenzen oder größere Düsendurchmesser erzielt. Die Tropfenerzeugersysteme zeichnen sich durch einen sehr geringen Volumenfehler aus. Dem gegenüber steht allerdings der Nachteil des eingeschränkten Viskositätsspektrums der Klebstoffe von maximal $20 \mathrm{mPa} \cdot \mathrm{s}$. Durch Wärmeeintrag in das Dosiersystem kann der Viskositätsbereich erhöht werden (Dilthey, Brandenburg und Schleser, 2005). Ein weiteres berührungsloses und tropfenweises Auftragsverfahren stellen Ventildosiersysteme dar (siehe Abb. 12 (e)). Meist werden hier piezoelektrisch betriebene Nadelventile eingesetzt, die ein Klebstoffreservoir freigeben. Dabei steht das Reservoir unter Druck.

Oben genannte Techniken gehören zu den meist eingesetzten Klebstoffauftragsverfahren in Mikromontageanlagen. Darüber hinaus gibt es noch weitere Verfahren für den Klebstoffauftrag im Umfeld der Mikrosystemtechnik, welche aber nicht in Mikromontageanlagen eingesetzt werden. Beispiele hierfür sind das Spin Coating, der Einsatz von sog. Spacer Beads (Abstandskügelchen in den Klebstoff beigemischt), die Sprühbelackung, das Dippen des Substrates in Klebstoff oder auch der Einsatz von Klebefolien.

\section{$\square$ Substrat / Werkstück $\cong$ Auftragswerkzeug $\square$ Klebstoff}

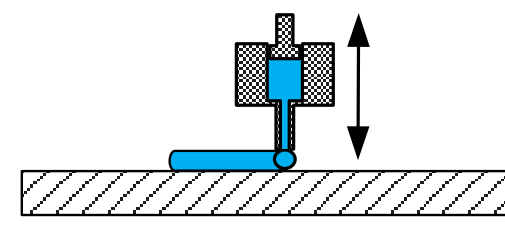

(a)

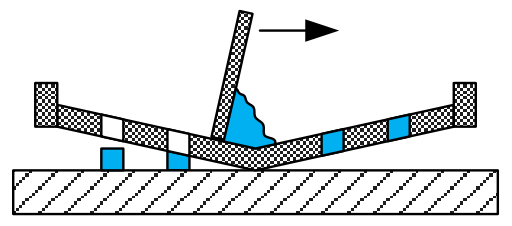

(b)

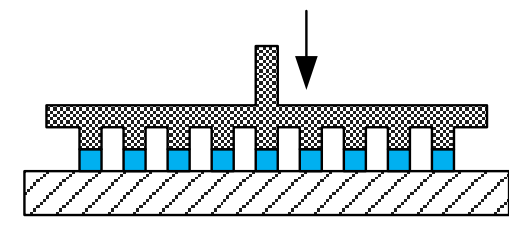

(c)

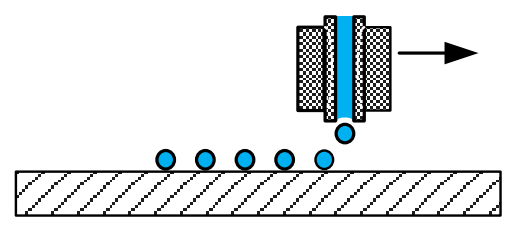

(d)

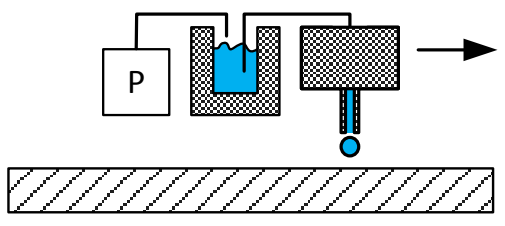

(e)

Abb. 12: Schematische Darstellung der Klebstoffauftragsverfahren in Mikromontageanlagen. (a) Kanülendispenser, (b) Siebdruck, (c) Pin-Transfer-Technik, (d) Tropfenerzeugung und (e) Ventildosierer. Angelehnt an Dilthey, Brandenburg und Schleser (2005) sowie Krebs (2009). 


\subsection{Zusammenfassende Betrachtung des Stands der Technik}

Für die Fertigung von Funktionsmodellen in geringen Stückzahlen für die Mikrosystemtechnik werden sowohl Mikrostrukturierungs- als auch Mikromontageoperationen benötigt. Nachfolgend erfolgt eine zusammenfassende Betrachtung zu dem Stand der Technik in diesen Disziplinen.

Die verwendeten Basisprozesse für die Mikrostrukturierung von Substraten für Mikrosysteme, wie bspw. thermische Oxidation, physikalische und chemische Gasphasenabscheidung, Photolithographie sowie trocken- und nasschemische Ätzung, werden aus der mikroelektronischen Fertigungstechnik übernommen. Hierbei wird auf eine langjährige Erfahrung und auch auf die Möglichkeit der Batchprozessierbarkeit zurückgegriffen. Neben den genannten Basisprozessen werden auch Nebenprozesse, wie bspw. Reinigungs- oder auch Temperierungsverfahren, benötigt. Daher ergibt sich für die Mikrostrukturierung von Substraten für Mikrosysteme eine wiederholende Prozessfolge aus Schichtabscheidung, Photolithographie und Schichtätzung, wobei stets auf Schichtgegebenheiten, wie bspw. Selektivität oder auch Kontamination vorhandener Schichten mit Ätzmitteln, zu achten ist.

Die Vielzahl der notwendigen Prozessschritte für die Mikrostrukturierung begründet auch die hohe Anzahl der notwendigen Geräte, wie bspw. Mask Aligner, Oxidationsöfen, Abscheideanlagen oder auch Spin Coatinganlagen. Die Fertigung muss in Reinräumen erfolgen, so dass die Anzahl von Partikeln reduziert wird und die Fertigung des Mikrosystems bzw. auch die spätere Funktionsfähigkeit hierdurch nicht negativ beeinflusst wird.

Neben der Mikrostrukturierung von Substraten ist die Mikromontage ein weiterer Bestandteil in der Mikrofertigung von Mikrosystemen. Dabei setzt sich ein Mikromontageprozess aus den Vorgängen des Handhabens und des Fügens zusammen.

Für das Handhaben sind vorstehend die möglichen Arten von Mikromontageanlagen vorgestellt worden. Diese werden anhand ihres Automatisierungsgrades in manuell, teleoperiert und automatisiert unterteilt. Vor allem für kleine Stückzahlen, wie bspw. für Kleinserien und Funktionsmodelle, werden die manuellen und teleoperierten Anlagen eingesetzt. Bei manuellen Systemen erfolgt der komplette Prozess manuell; dies bedeutet, dass der Operator direkt die Bauteile mit Werkzeugen bewegt. Teleoperierte Systeme zeichnen sich hingegen aus, dass die Bewegungen des Operators über Eingabegeräte direkt mit Teleoperatoren im Arbeitsraum ausgeführt werden. Bei höheren zu fertigenden Stückzahlen werden automatisierte Mikromontageanlagen eingesetzt. Allen Systemen wiederum gemein ist, dass viele unterschiedliche Werkzeuge, wie bspw. Vakuumgreifer, unterschiedlich aktuierte Zweibackengreifer und Beobachtungseinheiten, verwendet werden können.

Als eines der meist eingesetzten Fügeverfahren in der Mikrosystemtechnik ist das Kleben vorgestellt worden. Dieses wird vor allem auch für die Fügung von piezoelektrischen Aktoren mit Substraten, um bspw. Pumpen, Druckköpfe oder auch Sensoren herzustellen, eingesetzt. Hierfür steht eine Vielzahl an Klebstoffauftragsverfahren bereit, welche zum einen hinsichtlich ihrer Auftragsgeometrie und zum anderen hinsichtlich ihres Abstandes zum Substrat unterteilt werden können. Eines der meist eingesetzten Verfahren in Mikromontageanlagen sind Kanülendispenser, welche punkt- und auch linienförmige Auftragsmuster erzeugen. 


\section{Kritik am Stand der Technik}

Für die Herstellung von Mikrosystemen werden sowohl Mikrostrukturierungs- als auch Mikromontageoperationen benötigt. Kapitel 2 zeigt hierzu den Stand der Technik auf. Allerdings ergeben sich hieraus für die Fertigung von Funktionsmodellen / Redesigns in geringen Stückzahlen für die Mikrosystemtechnik Defizite, welche nachfolgend aufgelistet werden.

Die Defizite in der Mikrostrukturierung von Substraten für Funktionsmodelle sind:

Lange Prozesszeit: Die Mikrostrukturierung von Substraten besteht aus einer wiederholenden Prozessfolge aus Schichtabscheidung, Photolithographie und Schichtätzung. Neben den Basisprozessen sind auch Nebenprozesse, wie bspw. Reinigungs- und Tempervorgänge, immer notwendig. Unterschiedlich tief abzutragende Strukturen in einem Substrat durchlaufen jeweils die alternierende Prozessfolge. Diese Herangehensweise resultiert je nach Komplexität der Formen in einer langen Prozesszeit (Fujita, 2007; Benilov et al., 2008; Pimpin und Srituravanich, 2011). Moon et al. (2008) und Kim et al. (2010) zeigen hierzu jeweils beispielshaft das mehrmalige Durchlaufen der Prozessfolge für die Mikrostrukturierung der fluidmechanischen Substratbasis eines piezoelektrisch betriebenen Mikrotropfenerzeugers. Es ist nicht möglich, die Mikrostrukturierung eines Funktionsmodelles für Voruntersuchungen in kurzer Zeit vorzunehmen.

Hohe Kosten bei geringen Stückzahlen: Mikrostrukturierungsverfahren in der Mikrotechnik stammen aus der Mikroelektronik, welche sich durch ihre Batchprozessierbarkeit auszeichnen. Es werden neben einer Vielzahl an Gerätschaften (Mask Aligner, Ätzanlagen etc.), unterschiedlichen Chemikalien (Ätzlösungen, Photoresist etc.), Maskensätzen auch ein Reinraum benötigt. Dadurch entstehen enorme Kosten (Yeon und Park, 2007; Hwang et al., 2009). Lawes (2007) und Schwesinger (2015) nennen hierzu Anschaffungskosten von bspw. Trockenätzanlagen nach dem Bosch-Prozess (DRIE) in Höhe von mind. 500 T€, Mask Alignern von mind. $200 \mathrm{~T} €$ sowie von Spin Coatern von mind. $50 \mathrm{~T} €$. Die hohen Kosten relativieren sich nur bei einer hohen Anzahl in Batchprozessen gleich gefertigter Mikrosysteme (Abgrall und Gué, 2007; Fujita, 2007; Ashida et al., 2010). Bei geringen Stückzahlen erfolgt keine Kostenreduktion, so dass sich eine kostenintensive Fertigung von Funktionsmodellen ergibt.

Technisch komplexe Prozesse / geringe Individualität: Die Mikrostrukturierung ist ein technisch äußerst komplexer Prozess. Es müssen sowohl Material- als auch Prozessparameter aufeinander abgestimmt werden (Lazar, Grym und Foret, 2006), um definierte Ätzprofile, hohe Selektivitäten, hohe Ätzraten und geringe Substratkontaminationen zu erhalten. Die Vielzahl der notwendigen Prozessschritte birgt auch ein höheres Fehlerrisiko. Jedes Mikrosystem bzw. jede Mikrostrukturierungsoperation benötigt unter Beachtung von Material- und Prozessparametern seinen eigenen Prozessablauf (Rebello, 2004; Brousseau, Dimov und Pham, 2010). Hieraus ergibt sich, dass unterschiedliche Dimensionen bzw. Formen aufgrund meist notwendiger Prozessanpassungen nicht leicht umsetzbar sind.

Hoher Schulungsaufwand: Operatoren für Mikrostrukturierungsaufgaben müssen nicht nur in der Bedienung komplexer Gerätschaften geschult sein, sondern auch im Umgang mit giftigen bzw. ätzenden Chemikalien und deren Entsorgung. Darüber hinaus müssen sie über ein profundes mikrotechnologisches Fachwissen verfügen, um die einzelnen Prozesse und Mate- 
rialien aufeinander abzustimmen (Fujita, 2007; Benilov et al., 2008; Hwang et al., 2009). Im Ergebnis bedeutet dies, dass ein erhöhter Schulungsaufwand der Operatoren notwendig ist.

Für die Mikromontage von kleinen Stückzahlen können sowohl manuelle als auch teleoperierte Anlagen eingesetzt werden. Die Defizite bei der Mikromontage von Komponenten und Funktionswerkstoffen für Funktionsmodelle, welche in geringen Stückzahlen verlangt werden, sind hierbei:

Kein Einsatz als reines Fertigungshilfsmittel: Aufgrund der konstanten Miniaturisierung von Mikrosystemen bedarf es der Montage von immer kleineren Komponenten. Daraus folgt, dass auch bei Mikromontageanlagen der Fokus auf das Greifen kleiner Bauteile mit Abmessungen im Mikrometerbereich, das Positionieren mit Genauigkeiten im Nanometerbereich und damit verbunden auch auf die Überwachung des Montagevorganges mit stark vergröBernden und hochauflösenden Beobachtungseinheiten gelegt wird (Agnus, Nectoux und Chaillet, 2005; Ge, Gaines und Nelson, 2005; Cecil, Powell und Vasquez, 2007; Bogue, 2011). Jedoch werden für die Herstellung von Mikrosystemen auch Komponenten mit makroskopischen Bauteildimensionen, wie bspw. Substrate, benötigt. Das Greifen, das Positionieren und das Fügen dieser Bauteile mit Genauigkeiten im Mikrometerbereich ist ebenfalls ein essentieller Bestandteil in der Fertigung von Mikrosystemen. Alle genannten Systeme besitzen ihre Berechtigung bei Montagen von Bauteilen im Mikrometerbereich, allerdings nicht als direktes Fertigungswerkzeug auf Substratebene. Hier gilt es, auch planare Substrate mit makroskopischen Dimensionen zu greifen, zu positionieren und vor allem auch mit anderen Substraten zu fügen. Ein Beispiel hierfür ist die Klebung piezoelektrischer Substrate auf Membranen, um einen Membranaktuator zu formen.

Relativpositionierung mit hohen Kosten: In vielen Montagen werden neben dem reinen Absetzen von Bauteilen an gekennzeichneten Positionen (bspw. durch Justagemarkierungen) auch Relativpositionierungen von Bauteilen, d. h. in einem fest definierten Abstand von anderen Objekten auf dem Substrat, benötigt. Diese Aufgabe wird bislang mit motorbetriebenen Manipulatoren, welche wiederum eine Steuerung und Eingabegeräte verlangen, umgesetzt. Hierdurch entstehen höhere Kosten bezogen auf die eingesetzte Hardware; diese Verfahrensweise eignet sich damit nicht für die Herstellung von kostengünstigen Funktionsmodellen für die Mikrosystemtechnik in geringen Stïckzahlen.

Kein ganzflächiger und variierbarer Klebstoffauftrag: Auch bei den integrierten Klebstoffauftragstechniken in Mikromontageanlagen zeigt sich, dass deren Hauptanwendung die Fügung kleinster Komponenten ist. Techniken, wie bspw. Siebdruck, Dispensen, Tropfenerzeugung oder auch Stempeln, rufen sowohl linien- und punktförmige als auch nicht geschlossene Klebstoffoberflächen über eine größere Fläche hervor (Gaugel, Bengel und Malthan, 2004; Livelo und Rojas, 2008; Krebs, 2009). Für die Verbindung von Bauteilen auf Substratebene, wie bspw. die Klebung piezoelektrischer Keramiken auf Substraten, werden dünne, ganzflächige und homogene Klebstoffschichten benötigt, welche mit o. g. Techniken nicht unmittelbar realisierbar sind. Des Weiteren zeigen die Verfahren, dass unterschiedliche Klebschichtdicken nicht ohne weitere Kosten und Untersuchungen umsetzbar sind. Beispielsweise bestimmt bei dem Siebdruck die Dicke des Siebes die resultierende Klebschichtdicke. Unterschiedliche Klebschichtdicken erfordern somit unterschiedlich hohe Siebe. 


\section{Aufgabenstellung}

Die Aufgabe dieser Arbeit ist es daher, individuelle Funktionsmodelle / Redesigns eines Mikrosystems in geringen Stückzahlen und gleichzeitig in kurzer Zeit zu fertigen, so dass bereits in frühen Phasen der Entstehung des Mikrosystems die geforderten Eigenschaften des Produktes abgesichert sind. Als Anwendungsbeispiel dient ein Mikrotropfenerzeuger.

Mikrosysteme bestehen aus einem strukturierten Siliziumsubstrat und mindestens aus einem hierauf montierten Funktionswerkstoff. Bezogen auf das Anwendungsbeispiel eines Mikrotropfenerzeugers sind bspw. die Fluidkammern und die Düsen in dem Substrat eingelassen und ein piezoelektrischer Wandler wird über eine Klebschicht hierauf montiert. Hieraus ergeben sich zwei zu lösende Aufgaben für die Mikrofertigung der Funktionsmodelle: die individuelle Mikrostrukturierung von Substraten und die Mikromontage zweier Komponenten.

Die Mikromontage und die Mikrostrukturierung ergeben die Mikrofertigung des Mikrosystems. Die übergeordneten Aufgaben an die Mikrofertigung sind:

- Einsatz von Basisprozessen / Werkzeugen, die weder die Umgebung noch die maskenbasierte wiederholende Prozesskette mikroelektronischer Fertigungstechnik benötigen

- Herstellung fünf gleich gefertigter Funktionsmodelle in weniger als 24 Stunden, um rasch ein Redesign eines Mikrosystems mit ausreichend hoher Stückzahl zu prüfen

An die Mikrostrukturierung von Siliziumsubstraten werden folgende Aufgaben gestellt:

- Strukturierung von Freiformen mit einem absoluten Fehler von maximal $10 \mu \mathrm{m}$

- Ausschnitte von Freiformen mit einem absoluten Fehler von maximal $10 \mu \mathrm{m}$

- Erstellung von Kanälen mit senkrechten Seitenwänden und geringer Rauheit (Rauhtiefe kleiner $4 \mu \mathrm{m}$ ), mit einer minimalen Breite von $50 \mu \mathrm{m}$ und mit einem absoluten Fehler von maximal $5 \mu \mathrm{m}$. Die Breite, Länge und Tiefe der Kanäle muss variierbar sein

Mit Hilfe der Mikromontage wird der Funktionswerkstoff mit der strukturierten Basis gefügt. Es muss hierfür ein Montageplatz sowohl für den Klebstoffauftrag auf Substraten als auch für das Absetzen von Bauteilen erstellt werden. Dabei ergeben sich folgende Aufgaben:

- Relativpositionierungen sind mit einem absoluten Fehler von maximal $20 \mu \mathrm{m}$ möglich

- Für die Mikrosystemtechnik müssen Klebschichten auf einem Substrat mit einer Dicke von bis zu $20 \mu \mathrm{m}$ über eine Fläche von mindestens $10 \mathrm{~cm}^{2}$ bei einem absoluten Fehler von maximal $3 \mu \mathrm{m}$ aufgebracht werden

- Das Montagesystem enthält eine Heizmöglichkeit, um die Aushärtezeit von Klebstoffen zu reduzieren. Das System wird modular gestaltet, zeichnet sich durch eine intuitive Bedienbarkeit aus und es werden keine kostenintensiven motorbetriebenen Manipulatoren eingesetzt 


\section{Erwartete Vorteile}

Durch die erfolgreiche Bearbeitung dieser Aufgabe werden folgende Vorteile erwartet:

Angepasste und kostengünstige Fertigung geringer Stückzahlen: Es werden Verfahren für die angepasste und kostengünstige Fertigung von Mikrosystemen in geringen Stückzahlen zur Verfügung gestellt. Sowohl die Mikrostrukturierungs- als auch die Mikromontageoperationen werden mit einer ausreichend hohen Genauigkeit erfolgen. Es werden keine Massenfertigungstechniken aus der Mikrotechnik eingesetzt. Die Kosten für eine individuell an das jeweilige Mikrosystem angepasste Fertigungstechnik werden sich reduzieren. Darüber hinaus werden die Mikrostrukturierungstechniken nicht nur für die Strukturierung von Substraten für hybride, sondern auch für monolithische Mikrosysteme Anwendung finden. Es besteht nicht nur die Möglichkeit, Funktionsmodelle für Voruntersuchungen, sondern auch Produkte in Kleinserien zu fertigen. Zudem wird die Individualität eines Mikrosystems steigen. Die Fertigungstechnik erlaubt, beliebige Freiformen / Strukturen in Substraten zu erstellen, ohne bspw. auf kristallographische Gegebenheiten achten zu müssen.

Rasche Bewertung der Funktionsfähigkeit: Es wird die Möglichkeit geboten, in effizienter Weise Funktionsmodelle in den Produktentstehungsprozess von Mikrosystemen zu integrieren. Hierdurch können Fehler im Mikrosystementwurf zu einem frühen Zeitpunkt erkannt werden. Vor allem die unter Kapitel 1 beschriebene komplexe räumliche Integration der meist beweglichen Funktionskomponenten in Mikrosystemen, wie bspw. piezoelektrische Aktoren, kann früh auf ihre Funktion geprüft werden. Bei einem fehlerbehafteten Entwurf kann ein mögliches Redesign rasch abgeleitet werden. Die Fehler werden nicht erst nach der Etablierung eines spezifischen Fertigungsprozesses erkannt. Darüber hinaus werden EinwegProdukte für Analyseaufgaben zur Verfügung gestellt. So kann bspw. ein Druckkopf auf seine chemische Beständigkeit gegenüber bestimmten Fluiden bewertet werden.

Optimierung des Kalibrierprozesses: Kostengünstige und rasch fertigbare Funktionsmodelle erlauben, Kalibrierungsaufgaben an Mikrosystemen oder auch an Kompakt- / Minimalmodellen durchzuführen. Hiermit kann das Mikrosystem auf einen Betriebspunkt kalibriert werden. Bezogen auf das Anwendungsbeispiel der Tropfenerzeugung bedeutet dies, bspw. den Ausstoß eines vorgegebenen Tropfenvolumens eines Fluides mit bestimmten Eigenschaften (bspw. Oberflächenspannung) bei einer definierten Tropfenfluggeschwindigkeit zu erzielen. Es besteht die Möglichkeit, Aussagen zu treffen, welche Parameter im Design des Mikrosystems (bspw. Düsenquerschnitt) zu verändern sind, um die Ergebnisparameter zu erzielen.

Erschließung neuer Märkte: Die angepasste und kostengünstige Fertigungstechnik kann die Erschließung neuer Märkte für Mikrosysteme ermöglichen. Die großen Hindernisse, Investitionskosten als auch die komplexe Prozessführung zur Fertigung neuer Mikrosysteme, stehen nicht zentral im Vordergrund. Auch durch die Risiken eventueller Entwurfsfehler eines Funktionsmodelles wird das Bestreben der Erschließung neuer Märkte nicht gehemmt.

Verringerung des Schulungsaufwandes: Es werden Fertigungsverfahren zur Verfügung gestellt, die nicht auf der mikroelektronischen Fertigungstechnik basieren. Die Operatoren benötigen deshalb in geringem Maße das komplexe mikrotechnologische Fachwissen, um einzelne Prozessschritte aufeinander abzustimmen. 


\section{Abgrenzung}

Für die Fertigung von Funktionsmodellen für die Mikrosystemtechnik werden sowohl Mikrostrukturierungs- als auch Mikromontageoperationen durchgeführt. In vorliegender Arbeit werden hierfür Methoden zur Verfügung gestellt, welche nachfolgend genannte Aspekte nicht aufgreifen und sich somit wie folgt abgrenzen:

Angepasste Genauigkeit: Mikrostrukturierungsprozesse aus der Mikrotechnik erzielen Fertigungsgenauigkeiten von bis zu unter einem Mikrometer, da die Fertigungswerkzeuge aus der Mikroelektronik übernommen werden. Die Erzielung dieser Genauigkeitsklasse ist nicht Gegenstand dieser Arbeit, jedoch die rasche und individuelle Mikrostrukturierung von Siliziumsubstraten für Funktionsmodelle mit einer dafür ausreichend hohen Genauigkeit.

Bauteildimensionierung: Bei Mikromontagen steht das Handhaben und Fügen kleinster Bauteile mit Dimensionen im Mikrometerbereich im Vordergrund, so dass auch Genauigkeiten bei Positionieraufgaben von unter einem Mikrometer benötigt werden. Es ist nicht das Ziel, kleinste Bauteile im Mikrometerbereich zu montieren. Stattdessen steht vielmehr das Handhaben und Fügen von Funktionswerkstoffen, wie bspw. planare Substrate, mit Bauteildimensionen im Millimeterbereich im Vordergrund. Die Mikromontage von planaren Substraten wird nicht als separater Fertigungsschritt isoliert stehen, sondern wird direkt in den Prozessablaufplan bei der Fertigung von Funktionsmodellen für die Mikrosystemtechnik eingebunden. Hierbei steht die Verwendung einer intuitiv bedienbaren und kostengünstigen Mikromontageanlage als direktes Fertigungshilfsmittel mit ausreichend hoher Genauigkeit sowohl für Relativpositionierungen als auch für den Klebstoffauftrag im Fokus.

Materialauswahl: Die Mikrostrukturierungsoperationen werden an Silizium durchgeführt. Auch wenn es darüber hinaus noch eine Vielzahl weiterer Werkstoffe für die Mikrosystemtechnik gibt, wie bspw. Keramiken, Gläser oder auch Metalle, sind diese nicht Gegenstand vorliegender Arbeit. Die Fokussierung auf Silizium begründet sich dadurch, dass dieses der meist eingesetzte Werkstoff in der Mikrosystemtechnik ist, daraus folgernd eine bekannte Prozesstechnik für seine Weiterverarbeitung nach der Mikrostrukturierung existiert und somit eine ideale Substratbasis für Funktionsmodelle mit Seriencharakter darstellt.

Angepasste Stückzahlen: Charakteristisch für den Einsatz mikroelektronischer Fertigungstechnik ist die mögliche Batchprozessierbarkeit. In dieser Arbeit gilt es nicht, Fertigungsverfahren zur Verfügung zu stellen, welche eine kostengünstige Massenproduktion gleich gefertigter Mikrosysteme ermöglichen. Vielmehr geht es um die Herstellung von individuellen und kostengünstig gefertigten Funktionsmodellen für die Mikrosystemtechnik. Für die Durchführung von Experimenten / Optimierungsaufgaben wird eine ausreichende Anzahl von maximal fünf gleichzeitig gefertigten Funktionsmodellen bereitgestellt.

Anwendung: Vorliegende Fertigungstechnik, bestehend aus der Mikrostrukturierung und der Mikromontage, wird am Beispiel eines piezoelektrisch betriebenen Mikrotropfenerzeugers angewendet. Hierbei gilt es, die Eignung der vorgestellten Fertigungstechnik bzgl. einer raschen Herstellung von Funktionsmodellen mit ausreichend hoher Genauigkeit zu prüfen. Die Optimierung / Auslegung des Tropfenerzeugers bspw. hinsichtlich großer Tropfenvolumina ist nicht Gegenstand dieser Arbeit. 


\section{Eigener Ansatz}

\subsection{Mikrostrukturierung von Substraten}

\subsubsection{Werkzeuge für die Strukturierung von Substraten}

Die Strukturierungsarbeiten in der Mikrotechnik erfolgen sowohl in horizontaler als auch in vertikaler Richtung in dem Substratmaterial. Bezogen auf einen piezoelektrischen Tropfenerzeuger umfasst die Mikrostrukturierung in Siliziumsubstraten bspw. die Erstellung der fluidmechanischen Komponenten, wie Fluidzuführung als auch -verteilung, Drosseln, Pumpkammern und Düsen.

Die Motivation vorliegender Arbeit ist es, Techniken bereitzustellen, mit denen Funktionsmodelle für die Mikrosystemtechnik und hierbei insbesondere Tropfenerzeuger sowohl kosten- als auch zeitgünstig und gleichzeitig mit ausreichend hoher Präzision gefertigt werden. Darüber hinaus gilt es, eine flexible und somit individuelle Designumsetzung für die Mikrostrukturierungsarbeiten zu ermöglichen. Charakteristisch für die mikroelektronische Fertigungstechnik ist neben der alternierenden Prozessfolge auch die Forderung nach einem Reinraum. Diese Herangehensweise bei der Herstellung von Mikrosystemen ist vor allem für die Massenproduktion gleich gefertigter Systeme geeignet, allerdings nicht für die Fertigung einzelner individueller Funktionsmodelle. In diesem Zusammenhang stehen Schlagwörter wie bspw. Rapid Prototyping oder Rapid Manufacturing. Hierzu ist es wichtig, schnelle und kostengünstige Mikrostrukturierungsverfahren bereitzustellen, die mit geringem Aufwand die Herstellung einzelner individueller Funktionsmodelle ermöglichen, um die Eigenschaften eines Mikrosystems bereits in einer frühen Phase seiner Produktentstehung zu prüfen.

Für diese Anforderung bietet sich der Laserstrahlmaterialabtrag als ein geeignetes Bearbeitungsverfahren an. Die Vorteile der Materialbearbeitung mittels Laserstrahlung sind:

- Berührungslose und somit kräftefreie Bearbeitung auch von sehr harten und spröden Materialien

- Kleine Wärmeeinflusszone im Gegensatz zu anderen thermischen Strukturierungsverfahren wie bspw. Erodiertechniken

- Keine Einschränkung bzgl. der Formenvielfalt

- Designvariationen rasch mittels CAD Programmen (computer-aided design) und anschließendem Import in die Steuerung des Lasersystems umsetzbar

- Möglich hohe Materialvielfalt

- Keine Forderung nach einem Reinraum und Photolithographie inklusive wiederholender Prozessabfolge zur Strukturübertragung

Aus diesen Gründen wird für die Mikrostrukturierung von Freiformen und für Ausschnitte auf den Laserstrahlmaterialabtrag zurückgegriffen. Die Oberfläche eines laserabladierten Kanals 
weist aufgrund des thermischen Abtrages Poren und Schmelzaufwürfe auf. Als Resultat ergibt sich mitunter eine raue Kanalwand. Zusätzlich kommt es bei geringen Kanalbreiten auch zu Mehrfachreflektionen des Strahls an den Seitenflanken (Wolf, 2003). Als Ergebnis wird der Strahl stärker fokussiert und es entstehen nach unten spitz zulaufende Kanäle (siehe Abb. 13 (a)). Für die Erstellung der Pumpkammern, der Drosseln, der Fluidverteilung und der Fluidzuführung in einem Tropfenerzeuger stellt dieser Sachverhalt keinen erwähnenswerten Nachteil aufgrund der meist makroskopischen Dimensionen dar.

Allerdings gilt es, diesen Sachverhalt für Düsen in einem Tropfenerzeuger zu vermeiden, da zum einen der Kanalquerschnitt einen erheblichen Einfluss sowohl auf die Strömungsverhältnisse als auch auf die Tropfenparameter hat und zum anderen die Kanalrauheit die auftretenden Reibungsverluste während des Tropfenbildungsprozesses bestimmt. Daraus ergibt sich die Forderung nach einem Fertigungsverfahren, welches erlaubt, mikrofluidische Kanäle und insbesondere Mikrodüsen mit nahezu senkrechten und glatten Kanalwänden zu fertigen. Für diese Anforderung bietet sich das Trennschleifen mittels Wafersäge an. Wafersägen werden hauptsächlich zur Vereinzelung einzelner Dies ( $\hat{=}$ ungehäuste Halbleiterchips) aus der Waferbene heraus eingesetzt. Der Prozess wird dem Trennschleifen zugeordnet (DIN 8589-11, Stand September 2003). Je nach verwendetem Sägeblatt und Sägeparameter werden hierdurch glatte und nahezu senkrechte Kanäle erstellt (siehe Abb. 13 (b)). Fischer et al. (2008) geben für das Trennschleifen eine gemittelte Rauhtiefe $R_{z}$ von 1,6 bis $4 \mu \mathrm{m}$ bei normaler Prozessführung an. Bei genauer Prozessführung, bspw. durch die Wahl eines optimierten Sägeblattes bezogen auf das Substrat, ist eine gemittelte Rauhtiefe von bis zu 0,1 $\mu \mathrm{m}$ möglich. Es können nicht nur Substratmaterialien, sondern auch weitere Funktionswerkstoffe bearbeitet werden.

Zusammenfassend ist festzuhalten, dass die Mikrostrukturierung von Freiformen und von Ausschnitten in Siliziumsubstraten sowohl mittels Laserstrahlmaterialbearbeitung als auch mit dem Trennschleifen vorgenommen wird. Bezogen auf das Anwendungsbeispiel eines Tropfenerzeugers bedeutet dies, dass die fluidmechanischen Komponenten mit Ausnahme der Düsen mit dem Laserstrahl strukturiert werden. Hierdurch wird eine zeitgünstige Fertigung mit gleichzeitig hoher Präzision und hoher Formenvielfalt ermöglicht. Die Erstellung von mikrofluidischen Leitungskanälen, wie bspw. Düsen, erfolgt mittels Trennschleifen. Hierdurch werden nahezu senkrechte und glatte Kanäle ermöglicht. Vor dem Einsatz dieser Werkzeuge für die Fertigung von individuellen Funktionsmodellen müssen vorab jedoch umfassende Parameteruntersuchungen durchgeführt werden, wodurch eine hohe Formtreue bei einer gleichzeitig schnellen und effizienten Strukturierung gewährleistet wird. Die oben genannten Verfahren benötigen nicht das hohe prozesstechnische Umfeld aus der mikroelektronischen Fertigung. Es werden keine Reinräume verlangt und die Fertigungstechnik stellt sich nicht als eine Abfolge von wiederholenden Prozessen dar.

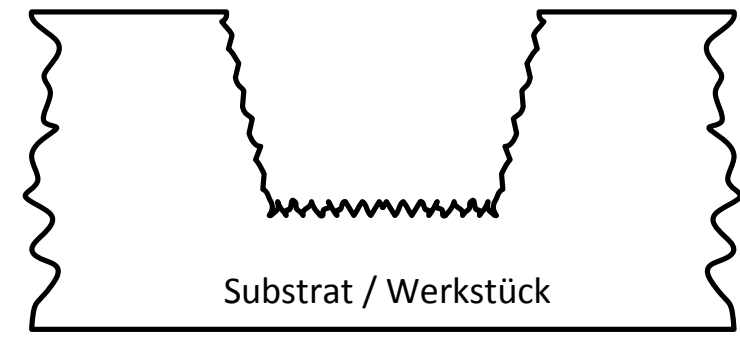

(a)

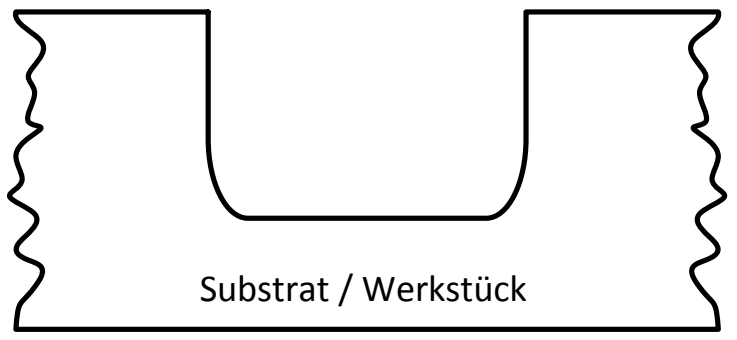

(b)

Abb. 13: Schematische Darstellung des Prozessergebnisses in (a) für die Laserstrahlmaterialbearbeitung mit dargestellter Rauheit an den Seitenflanken sowie am Boden und in (b) für das Trennschleifen mittels Wafersäge. 


\subsubsection{Grundlagen der Laserstrahlmaterialbearbeitung}

Die Laserstrahlmaterialbearbeitung ist ein thermisch sublimierendes Fertigungsverfahren, welches der Gruppe „Abtragen", in der Hauptgruppe „Trennen“, zugewiesen ist (DIN 8580, Stand September 2003; DIN 8590, Stand September 2003). Es werden meist gepulste Laser verwendet. Werden Laser mit Pulsdauern im Bereich von Nanosekunden eingesetzt, erfolgt der direkte Sublimationsabtrag. Hierbei wird die erforderliche Energie für den Materialabtrag mit einem fokussierten Laserstrahl geliefert, welcher den Werkstoff lokal so stark aufheizt, dass der Werkstoff an dieser Stelle aufschmilzt und bei Überschreiten der Verdampfungstemperatur verdampft. Abb. 14 zeigt die einzelnen Phasen des Laserstrahlsublimationsabtrages:

1. Absorption: Der fokussierte Laserstrahl trifft auf das Werkstück und liefert die notwendige Leistungsdichte ( $\triangleq$ Intensität) für den Materialabtrag

2. Wärmeleitung und Aufheizen: Durch Wärmeleitung erfolgt der Energietransport in das Material hinein

3. Aufschmelzen und Verdampfen: Ist die Schmelztemperatur des Werkstoffes erreicht, bildet sich ein Schmelzbad aus. Wird darüber hinaus auch die Verdampfungstemperatur überschritten, verdampft das Material

4. Schmelzaustrieb: Durch den entstehenden Dampfdruck wird das abladierte Material aus der Bearbeitungszone gedrückt. Am Rand der Laserstrahleintrittsseite bildet sich ein minimaler Schmelzaufwurf

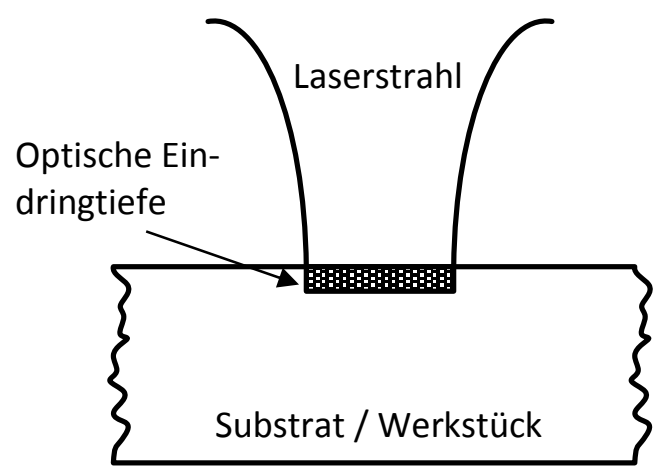

(a)

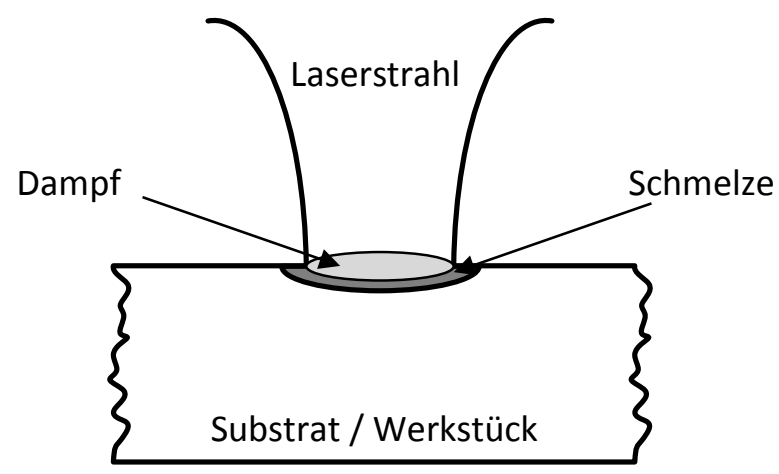

(c)

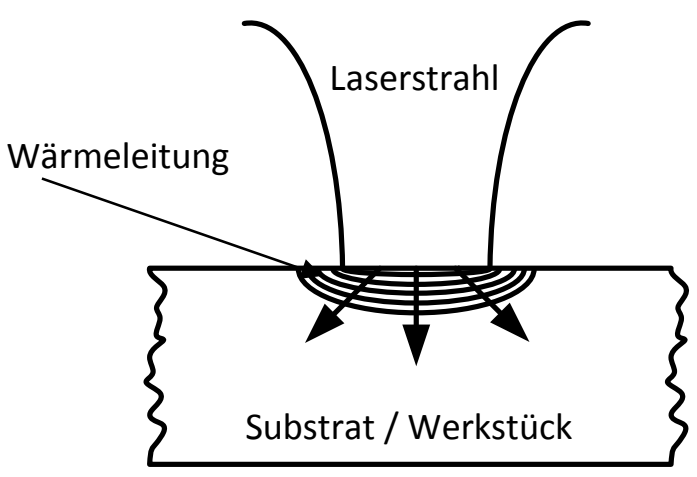

(b)

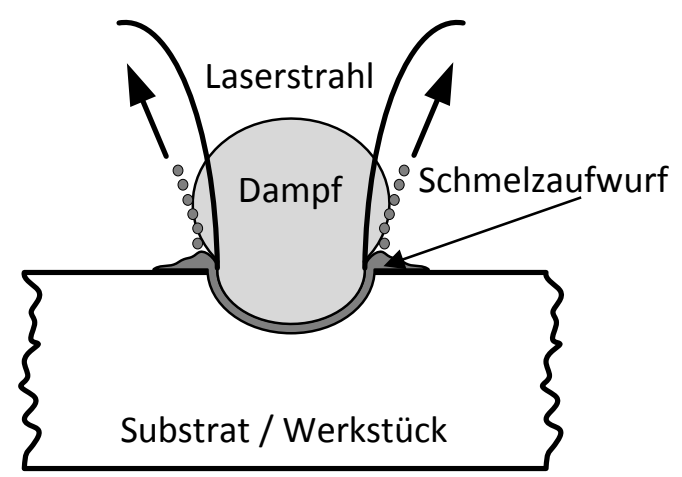

(d)

Abb. 14: Phasen des Sublimationsabtrages mittels Laserstrahlung. (a) Absorption der Laserstrahlung bis zur optischen Eindringtiefe in das Werkstïck, (b) Wärmeleitung, (c) Aufschmelzen mit Ausübung eines Schmelzbades und entstehendem Dampf und (d) Verdampfen mit Schmelzaustrieb. Angelehnt an Läßiger (1995) und Liess (2006). 
Durch den Einsatz von Ultrakurzpulslasern (Pulsdauern im Bereich von Femto- und Pikosekunden) werden gegenüber Kurzpulslasern deutlich höhere Pulsspitzenleistungen erzielt. Das Material verdampft schlagartig, bevor es die Umgebung aufheizt. Das Resultat ist, dass die Bandbreite der bearbeitbaren Werkstoffe erhöht wird und sich kein Schmelzaufwurf an der Strahleintrittsseite formiert. An dieser Stelle wird auch von einer kalten Ablation gesprochen.

Eine Laserstrahlquelle besteht im Wesentlichen aus einem laseraktiven Medium, einer optischen Pumpquelle und dem optischen Resonator. Dem laseraktiven Medium wird über die Pumpquelle Energie zugeführt. Hierdurch werden die enthaltenen Atome im laseraktiven Medium in energetisch höher gelegene Zustände gebracht. Dieser Zustand ist jedoch instabil, so dass das System wieder in den energetisch niedrigeren Zustand zurückfällt. Hierdurch werden Photonen freigesetzt, die die Energiedifferenz der beiden Zustände tragen. Dieser Prozess wird spontane Emission genannt. Um mehr Energie zu erzeugen, wird dieser Zustandswechsel stimuliert, anstatt spontan zu erfolgen. Hierbei erzwingt ein Photon die Emission eines weiteren Photons und es kommt zur Kettenreaktion. Ein Teil der freigesetzten Photonen trifft auf einen vollständigen reflektierenden Spiegel, der die Photonen wieder zurücklenkt und weitere Emissionen fördert. Dem gegenüber befindet sich ein teildurchlässiger Spiegel. Beide Spiegel bilden den sogenannten optischen Resonator. Ein Teil der Photonen tritt als Laserstrahl aus diesem Spiegel. Der Rest der Photonen wird wieder zurück in das laseraktive Medium reflektiert. Somit wird einem Photon bei mehrfachem Durchlaufen des laseraktiven Mediums die Möglichkeit geboten, die Emission weiterer Photonen zu stimulieren.

Die unterschiedlichen Lasertypen werden nach ihrem Medium unterschieden. Die zwei großen Gruppen sind zum einen Gaslaser (Excimerlaser, $\mathrm{CO}_{2}$ Laser ...) und zum anderen Festkörperlaser (Nd:YAG, Er:YAG ...). In der Mikrotechnik werden hauptsächlich Nd:YAG Festkörperlaser (Neodym-Yttrium-Aluminium-Garnet) eingesetzt (Brück, Rizvi und Schmidt, 2001; Liess, 2006; Bäuerle, 2009). Nd:YAG Laser emittieren bei einer Wellenlänge von 1064 nm im Infrarotbereich (IR). Durch die Möglichkeit, die Frequenz dieser Laser zu vervielfachen, werden Wellenlängen von 532, 355 oder $266 \mathrm{~nm}$ erzielt.

Die Wellenlänge bestimmt die Strukturauflösung und auch die Energieaufnahme im Werkstück. Daher gilt, je kürzer die Wellenlänge ist, desto höher ist die Strukturauflösung und desto höher ist die Fokussierbarkeit des Laserstrahls. Nd:YAG Laser werden sowohl im Dauerbetrieb als auch gütegeschaltet im Pulsbetrieb eingesetzt. Verhältnismäßig tragen die einzelnen Pulse in der kurzen Zeit nur wenig Material ab, allerdings wird dieser Effekt durch die hohen möglichen Pulsfrequenzen bis in den Kilohertzbereich kompensiert. Hierdurch wird eine gute Einkopplung der Laserstrahlung trotz bspw. einer sehr hohen Reflektivität des zu strukturierenden Substrates erzielt. Der Aufbau des optischen Resonators ist im Wesentlichen verantwortlich für die Ausbreitungs- und Fokussiereigenschaften des Laserstrahls. Hier entstehen Merkmale wie bspw. Moden und Divergenz.

Das Verhalten von Laserstrahlen wird im Allgemeinen mittels Gaußscher Strahlenoptik beschrieben, sofern die Laserstrahlung im Grundmode TEM $_{00}$ (transversalelektromagnetisch) angeregt und abgestrahlt wird. Allen Lasertypen ist gleich, dass der austretende Strahl durch einige wichtige Parameter beschrieben ist. Daher werden nachfolgend sowohl die Grundlagen der Gaußschen Strahlenoptik als auch Bearbeitungsparameter, welche nicht direkt von der Laserstrahlquelle abhängen, beschrieben. 


\subsubsection{Gaußsche Strahlenoptik}

Zur Beschreibung von Gaußschen Strahlen werden Zylinderkoordinaten verwendet. Dabei stellt die Ausbreitungsrichtung die Z-Achse dar; die Strahltaille bzw. der Fokus liegt im Koordinatenursprung an der Stelle $\mathrm{z}=0$.

Durch die Fokussierung des Laserstrahls werden im Fokus extrem hohe Intensitäten erzeugt. Dabei berechnet sich der Fokusdurchmesser aus dem doppelten Fokusradius $w_{0}$ nach (7.1).

$$
2 \cdot w_{0}=M^{2} \cdot \frac{4 \cdot f \cdot \lambda}{\pi \cdot D}
$$

Hierbei entspricht $\mathrm{f}$ der Brennweite der fokussierenden Optik, $\lambda$ der Wellenlänge, $\mathrm{D}$ dem Durchmesser des Strahles auf der Fokussieroptik und $\mathrm{M}^{2}$ der Beugungsmaßzahl. Die Beugungsmaßzahl beschreibt hierbei das Verhältnis zwischen dem realen und dem theoretischen Divergenzwinkel eines idealen Gaußschen Strahlprofils. Ursachen für diese Diskrepanz sind Amplituden- und Phasenstörungen, das Anschwingen höherer transversaler Moden oder auch die Ausbildung und Überlagerung von Teilstrahlen (Eichler und Eichler, 2006). Dabei gilt: Je kleiner die Beugungsmaßzahl ist, desto höher ist die Fokussiergüte des Laserstrahls.

Abb. 15 zeigt die axiale Ausbreitung eines Gaußschen Strahls in Z-Richtung. Der Strahlradius w(z) wird damit als Funktion der Entfernung z vom Fokuspunkt nach (7.2) bestimmt. $\mathrm{w}_{0}$ stellt den Minimalradius an der Strahltaille dar.

$$
w(z)=w_{0} \cdot \sqrt{1+\left(\frac{\lambda \cdot z}{\pi \cdot w_{0}^{2}}\right)^{2}}
$$

Eine weitere wichtige Größe in der Laserstrahlmaterialbearbeitung basierend auf der Gaußschen Strahlenoptik stellt die Rayleigh Länge $z_{R}$ dar. An dieser Stelle ist der Strahlradius $\mathrm{w}\left(\mathrm{z}=\mathrm{z}_{\mathrm{R}}\right)$ auf $\sqrt{2} \cdot \mathrm{w}_{0}$ aufgeweitet und definiert die Grenzen des Nahfeldes des Strahlprofils. Durch Einsetzen in (7.2) wird damit die Rayleigh Länge $z_{R}$ in (7.3) bestimmt.

$$
z_{R}=\frac{\pi \cdot w_{0}^{2}}{\lambda}
$$

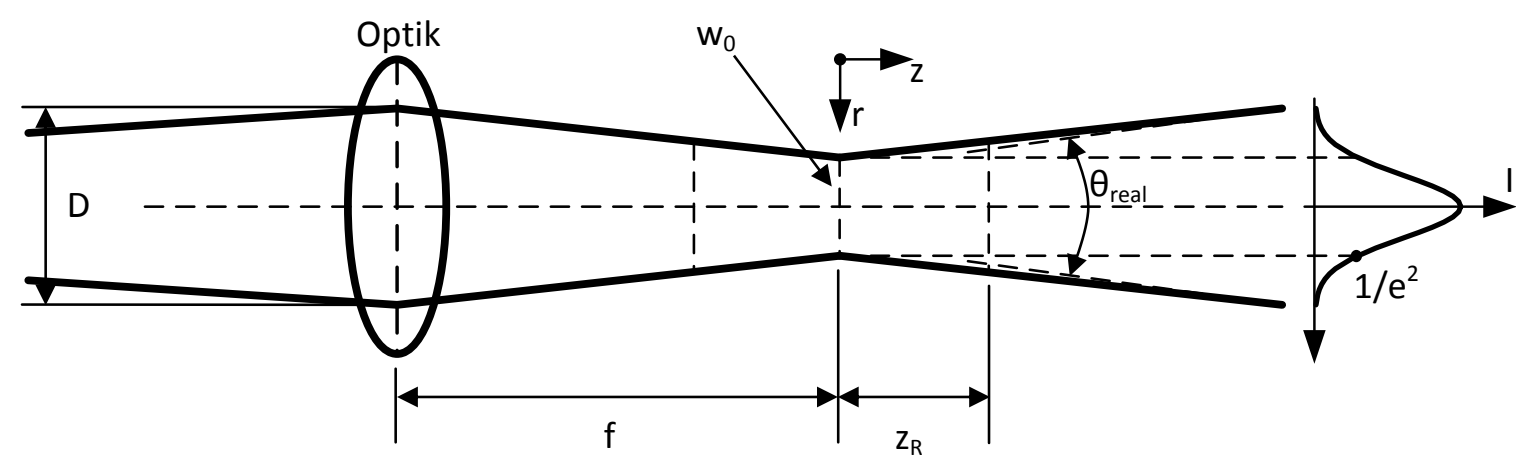

Abb. 15: Profil eines Gaußschen Laserstrahls. Hierbei entspricht D dem Durchmesser des Strahles auf der Fokussieroptik, $w_{0}$ dem Fokusradius, f der Brennweite der Fokussieroptik, $\mathrm{z}_{\mathrm{R}}$ der Rayleigh Länge und $\theta_{\text {real }}$ zeigt den realen Divergenzwinkel. Zusätzlich zu sehen ist die Intensitätsverteilung im Gaußschen Strahlprofil. Angelehnt an Liess (2006). 
Die örtliche Intensitätsverteilung I(r,z) besitzt eine Amplitude, die durch eine Gaußfunktion nach (7.4) beschrieben wird. Dabei ist der Fokusradius $\mathrm{w}_{0}$ als derjenige Strahlradius bestimmt, bei dem die Intensität auf $1 / \mathrm{e}^{2}(13,5 \%)$ abgefallen ist. $\mathrm{I}_{0}(\mathrm{z})$ stellt hierbei die maximale Intensität auf der Strahlachse bei $\mathrm{r}=0$ einer Wellenfront dar.

$$
I(r, z)=I_{0}(z) \cdot \exp \left(-\frac{2 \cdot r^{2}}{w^{2}(z)}\right)
$$

Mit größerem Abstand $\mathrm{z}$ von der Strahltaille nimmt die Intensität ab. $\mathrm{I}_{0}(\mathrm{z})$ wird nach (7.5) bestimmt. $\mathrm{I}_{0}(0)$ ist hierbei die maximale Intensität des gesamten Laserstrahls bei $\mathrm{r}=0$ und $\mathrm{z}=0$.

$$
I_{0}(z)=I_{0}(0) \cdot \frac{w_{0}^{2}}{w^{2}(z)}
$$

In (7.6) wird der Zusammenhang zwischen der Laserpulsleistung $\mathrm{P}_{\text {Puls }}$ und der maximalen Intensität $\mathrm{I}_{0}(\mathrm{z})$ einer Wellenfront für $\mathrm{r}=0$, welcher durch Integration von (7.4) aufgestellt wird (Eichler und Eichler, 2006), gezeigt.

$$
P_{P u l s}=\frac{\pi}{2} \cdot w^{2}(z) \cdot I_{0}(z)
$$

Durch Messung der mittleren Leistung $\mathrm{P}_{\text {Mittel }}$ wird die Pulsleistung $\mathrm{P}_{\text {Puls }}$ nach (7.7) berechnet. Hierbei wird noch die Pulsdauer $\tau_{\text {Puls }}$ und die Pulsfrequenz $f_{\text {Puls }}$ hinzugezogen. Abb. 16 verdeutlicht den Zusammenhang zwischen mittlerer Laserleistung $\mathrm{P}_{\text {Mittel }}$ und Pulsleistung $\mathrm{P}_{\text {Puls }}$.

$$
P_{\text {Puls }}=\frac{P_{\text {Mittel }}}{\tau_{\text {Puls }} \cdot f_{\text {Puls }}}
$$

Durch Gleichsetzen von (7.6) mit (7.7) wird die maximale Intensität $\mathrm{I}_{0}(0)$ nach (7.8) ermittelt.

$$
I_{0}(0)=2 \cdot \frac{P_{\text {Mittel }}}{\tau_{\text {Puls }} \cdot f_{\text {Puls }} \cdot \pi \cdot w_{0}^{2}}
$$

Die maximale Intensität $\mathrm{I}_{0}(0)$ hängt somit neben der mittleren Leistung auch von der Pulsdauer, der -frequenz und von dem Fokusradius ab. Hiermit ist auch das stetige Bestreben der photonischen Industrie nach der Verringerung der Pulsdauer begründet.

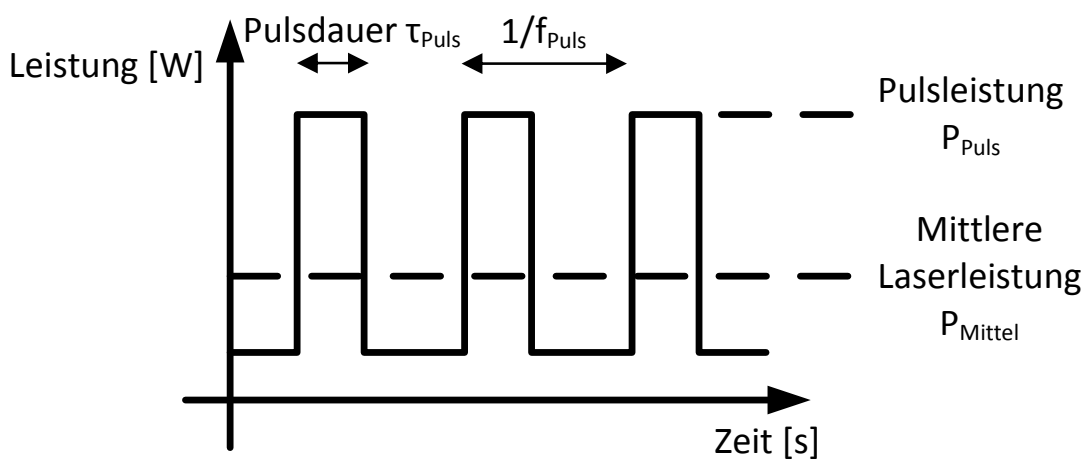

Abb. 16: Schematische Darstellung des zeitlichen Verlaufs der Pulsleistung $\mathrm{P}_{\text {Puls }}$ und der mittleren Laserleistung $\mathrm{P}_{\text {Mittel. }}$ Die Pulse werden durch Rechteckimpulse angenähert. 


\subsubsection{Laserstrahlquellen unabhängige Parameter für die Materialbearbeitung}

Eine der Motivationen für diese Arbeit ist es, den Einfluss einzelner Bearbeitungsparameter des Laserstrahlmaterialabtrages aufzuzeigen, welche unabhängig von der Laserstrahlquelle sind. Meist liegt der Fokus der Untersuchungen über den Einfluss einzelner Parameter auf solche Parameter, welche direkt der Laserstrahlquelle zugeordnet werden. Dies sind bspw. die Pulsdauer, die Pulsfrequenz oder auch die Pulsleistung (Meijer, 2004; Amer et al., 2005; Chien und Gupta, 2005; Ancona et al., 2009). Für viele bereits etablierte und fest eingestellte Lasersysteme besteht keine Möglichkeit, diese Größen nach Anschaffung zu variieren bzw. ohne größeren Aufwand, bspw. durch Neujustage des Lasersystems etc., zu ändern.

Vorliegend wird von einem Lasersystem ausgegangen, welches im Wesentlichen eine Laserstrahlquelle, eine Strahlführungseinheit und eine verstellbaren Höhenachse für die Fokuspositionierung enthält. Dabei gilt, dass die Strahlführungseinheit entweder aus einem Galvanometerscankopf, welcher den Laserspot auf dem Werkstück steuert, oder aus einem Laserstrahlaustragskopf, welcher zusätzlich auf einem Kreuztisch für die Laserspotbewegung montiert ist, besteht.

Im Detail wird nachfolgend der Einfluss der Fokusposition, der Vorschubgeschwindigkeit und auch des Linienabstandes beschrieben. Alle drei Parameter sind unabhängig von der Laserstrahlquelle und dem damit verbundenen physikalischen Ablationsprozess. Die Parameter finden sowohl bei Schneidprozessen als auch bei einem ebenenweisen Materialabtrag mittels Laserstrahlung Anwendung. Für den vorliegenden Membrandruckkopf bedeutet dies, dass der Schneidprozess Anwendung bei der Erstellung der Fluidzuführung und der ebenenweise Materialabtrag bei der Erstellung der Drosseln, Pumpkammern und Fluidverteilung Anwendung finden werden.

\subsubsection{Fokusposition}

Einen grundlegenden Parameter für die Laserstrahlmaterialbearbeitung stellt die Position des Fokus in Relation zu dem Werkstück dar. Voraussetzung, um Material abzutragen ist, dass die eingebrachte Intensität des Laserstrahles in das Werkstück größer ist als die materialspezifische Schwellintensität. In (7.5) wird gezeigt, dass die Intensität $\mathrm{I}_{0}(\mathrm{z})$ einer Wellenfront des Laserstrahles sowohl von der maximalen Intensität des gesamten Laserstrahles $\mathrm{I}_{0}(0)$ als auch von dem Strahlradius w(z) bestimmt wird. Laserstrahlen nach Gaußscher Strahlenoptik werden in ein Nah- und in ein Fernfeld unterteilt. Dabei stellt die Rayleigh Länge $\mathrm{z}_{R}$ die notwendige Grenze dar. In dem Bereich $0<\mathrm{z}<\mathrm{z}_{\mathrm{R}}$ befindet sich das Nahfeld; $\mathrm{z}>\mathrm{z}_{\mathrm{R}}$ definiert den Bereich des Fernfeldes. Für den Strahlradius an der Position der Rayleigh Länge gilt, dass sich dieser um den Faktor $\sqrt{2}$. gegenüber dem Strahlradius im Fokus vergrößert. Das Resultat hieraus ist, dass sich die Intensität des Strahles an der Stelle $\mathrm{z}=\mathrm{z}_{\mathrm{R}}$ gegenüber der maximalen Intensität des gesamten Strahls $\mathrm{I}_{0}(0)$ halbiert. (7.9) zeigt diesen Zusammenhang auf.

$$
\begin{aligned}
& I_{0}\left(z=z_{R}\right)=I_{0}(0) \cdot \frac{w_{0}^{2}}{w^{2}\left(z=z_{R}\right)} \\
& \operatorname{mit} w^{2}\left(z=z_{R}\right)=2 \cdot w_{0}^{2} \\
& I_{0}\left(z=z_{R}\right)=\frac{1}{2} \cdot I_{0}(0)
\end{aligned}
$$


Als Zwischenergebnis ist damit festzuhalten, dass die Position des Fokus in Relation zu dem Werkstück großen Einfluss auf die eingebrachte Intensität und somit auch auf die Effizienz des Materialabtrages besitzt. Idealerweise wird bei modernen Systemen die Möglichkeit geboten, den Fokus nach einer definierten Zeit oder nach einer fest definierten Anzahl an Überfahrungen des Laserspots über das Werkstück automatisch nachzujustieren. Hierbei wird entweder die motorbetriebene Höhenachse über die Strahlführungseinheit gesteuert oder die Strahlführungseinheit bietet selbst Möglichkeiten, den Fokus nachzujustieren. Dieser Vorgang wird auch als „focus shifting“ bezeichnet. Da allerdings nicht jedes Lasersystem diese Möglichkeit bietet, wird in den vorliegenden Untersuchungen während des gesamten Laserstrahlmaterialabtrages eine konstante Fokusposition in Relation zu dem Werkstück gewählt und deren Einfluss auf den Materialabtrag untersucht.

Die Intensitätsberechnung nach (7.5) wird durch den Strahlradius w(z) bestimmt. Wie bereits beschrieben, bedeutet eine Zunahme des Strahlradius eine Abnahme der Intensität. Damit verbunden, bestimmt die Fokuslage auch den Strahldurchmesser bzw. im Ergebnis den resultierenden materialspezifischen Bearbeitungsdurchmesser für den Materialabtrag. Abb. 17 verdeutlicht diesen Sachverhalt für verschiedene Fokuspositionen in Relation zu dem Werkstück. Um eine präzise Fertigung des Druckchips, vor allem bei dem ebenenweisen Materialabtrag der fluidmechanischen Komponenten zu gewähren, gilt es, neben einer effizienten Bearbeitung durch die optimale Position des Fokus im Werkstück auch den damit einhergehenden Bearbeitungsdurchmesser mit zu berücksichtigen.

Es ist ersichtlich, dass bspw. für einen ebenenweisen Laserstrahlmaterialabtrag nur ein Fokus nahe des zu abladierenden Gebietes auf der Werkstückoberfläche zu einem geringen Bearbeitungsdurchmesser mit gleichzeitig hohem Materialabtrag führen wird. Auf der anderen Seite ist für das Laserstrahlschneiden Voraussetzung, durch eine günstige Wahl der Fokusposition die eingebrachte Intensität in das Werkstück optimal zu verteilen, um das Werkstück durchzutrennen. Dies gilt es, für die Erstellung der Fluidzuführung zu berücksichtigen.

Dementsprechend besitzt die Fokusposition sowohl Einfluss auf den Strahl- / Bearbeitungsdurchmesser als auch damit verbunden auf die Intensität des Laserstrahles besitzt. Generell gilt, je kleiner dieser Durchmesser ist, desto höher ist die Intensität und desto schmälere bzw. feinere Formen werden erstellt.

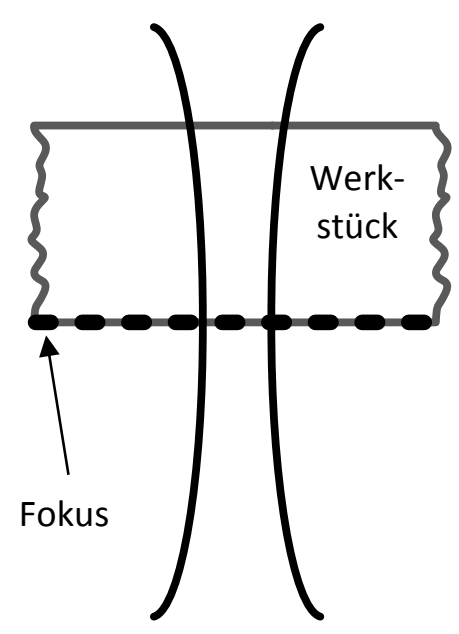

(a)

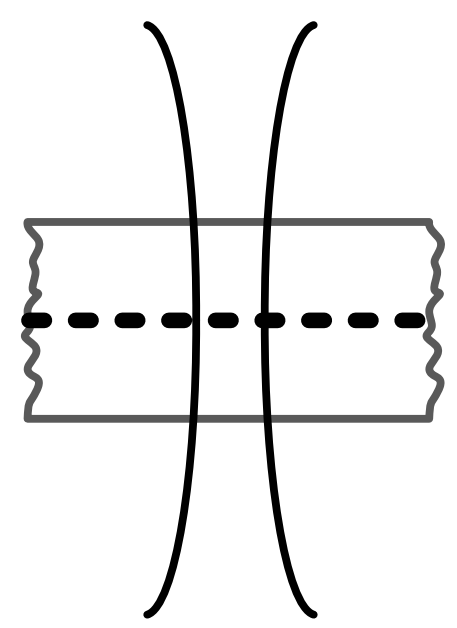

(b)

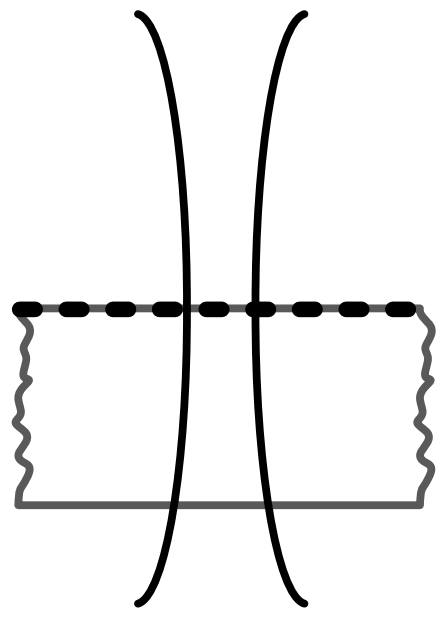

(c)

Abb. 17: Schematische Darstellung verschiedener Fokuspositionen. Fokus in (a) auf der Unterseite des Werkstïckes, (b) in der Mitte des Werkstückes und in (c) auf der Oberseite des Werkstïckes. Angelehnt an Kagerer, Irlinger und Lueth (2011). 


\subsubsection{Vorschubgeschwindigkeit}

Einen weiteren Laserstrahlquellen unabhängigen Parameter für die Laserstrahlmaterialbearbeitung stellt die Vorschubgeschwindigkeit $v_{S}$ des Laserspots dar. Zusammen mit dem materialspezifischen Bearbeitungsdurchmesser $\mathrm{d}_{\mathrm{W}}$, der Pulsperiodendauer $\mathrm{t}_{\text {Puls }}(\hat{=}$ reziproke Pulsfrequenz $\mathrm{f}_{\text {Puls }}$ ) und der Pulsdauer $\tau_{\text {Puls }}$ wird der Pulsüberlapp PÜ in (7.10) nach DIN 32540 (Stand August 2012) definiert.

$$
P \ddot{U}=1-\frac{v_{S} \cdot t_{P u l s}}{d_{W}+v_{S} \cdot \tau_{P u l s}}
$$

Dabei gibt der Pulsüberlapp an, in welchem prozentualen Maß sich die aufeinanderfolgenden Laserpulse überdecken (siehe Abb. 18). Die enthaltene Pulsverzerrung $\mathrm{v}_{\mathrm{S}} \cdot \tau_{\text {Puls }}$ kann aufgrund ihres geringen Einflusses vernachlässigt werden. Selbst bei Pulslängen im Bereich von $200 \mathrm{~ns}$ beträgt die Pulsverzerrung lediglich $20 \mathrm{~nm}$ bei einem Vorschub von $100 \mathrm{~mm} / \mathrm{s}$. Hierdurch kann der Pulsüberlapp PÜ wie folgt zu (7.11) vereinfacht werden.

$$
P \ddot{U}=1-\left.\frac{v_{S} \cdot t_{P u l s}}{d_{W}+v_{S} \cdot \tau_{P u l s}}\right|_{v_{S} \cdot \tau_{P u l s} \cong 0}=1-\frac{v_{S} \cdot t_{P u l s}}{d_{W}}=1-\frac{v_{S}}{d_{W} \cdot f_{P u l s}}
$$

Hohe PÜs bzw. eine damit verbundene geringe Vorschubgeschwindigkeit führen dazu, dass mehr Energie an fast gleicher Stelle im Werkstück eingebracht wird. Das Ergebnis ist, dass dadurch mehr Material pro Überfahrung abgetragen wird. Allerdings gilt es, auf die Prozesszeit zu achten. Der Grundgedanke vorliegender Arbeit bei der Strukturierung von Siliziumsubstraten ist es, nicht möglichst viel Material in wenig Überfahrungen abzutragen, sondern in möglichst kurzer Zeit. Darüber hinaus gilt es, den Einfluss des Vorschubes auf die Rauheit der abladierten Bereiche und auch auf den Bearbeitungsdurchmesser zu untersuchen. Ein hoher PÜ bedeutet, dass das verdampfte Material weniger Platz hat, aus der Bearbeitungsfuge zu entweichen, ohne dabei von einem darauffolgenden Puls getroffen zu werden.

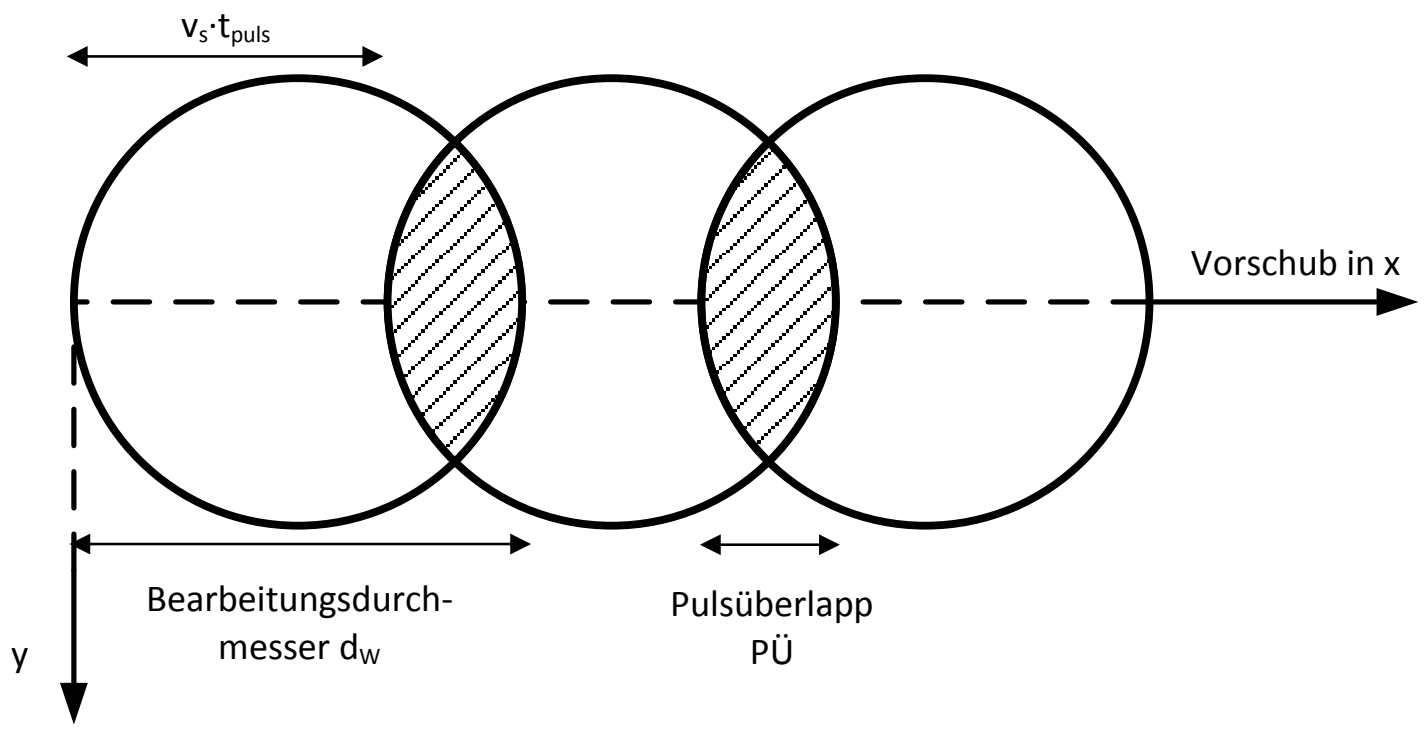

Abb. 18: Schematische Darstellung des Pulsüberlapps PÜ. Angelehnt an Kagerer, Irlinger und Lueth (2011) sowie DIN 32540 (Stand August 2012). 


\subsubsection{Linienabstand}

Sowohl für das Laserstrahlschneiden als auch für einen ebenenweisen Laserstrahlmaterialabtrag setzen sich die Schnitt- / Abtragsmuster aus mehreren parallel verlaufenden und auch überlappenden Laserlinien zusammen. Auf diesen Linien fährt im Ergebnis der Laserspot die Formen ab und trägt Material ab.

Der Abstand der Linien $\Delta \mathrm{y}$ und der materialspezifische Bearbeitungsdurchmesser $\mathrm{d}_{\mathrm{W}}$ bestimmen den resultierenden Spurüberlapp SÜ, welcher in (7.12) nach DIN 32540 (Stand August 2012) formal beschrieben ist. Abb. 19 zeigt den SÜ auf. Je größer der Abstand der Linien ist, desto kleiner ist der SÜ.

$$
S \ddot{U}=1-\frac{\Delta y}{d_{W}}
$$

Bei der Wahl des Laserstrahlsublimationsabtrages schwächt der abladierte entgegenströmende Materialdampf den eintreffenden Laserstrahl in der schmalen Bearbeitungsfuge ab. Diesem Umstand kann entgegengewirkt werden, indem die Bearbeitungsfuge unter dem Einsatz mehrerer Schnitt- / Schraffurlinien verbreitert wird. Allerdings gilt auch hier wiederum, dass der resultierende Spurüberlapp sowohl die Abtragsrate als auch die Rauheit des bearbeiteten Gebietes beeinflusst. Wie auch bei dem Pulsüberlapp ist deshalb auf die Prozesszeit zu achten, da selbst durch den Einsatz mehrerer Linien die Bearbeitungsfuge vergrößert wird; gleichwohl ist keine zeiteffiziente Laserstrahlmaterialbearbeitung hierdurch gegeben.

An dieser Stelle wird das Ergebnis gewonnen, dass vorliegend nur die Bearbeitungsstrategie und nicht Parameter der Laserstrahlquelle den Ablationsprozess beeinflussen werden. Die Fokusposition, der Vorschub und auch der Linienabstand können nahezu bei jeder Laserstrahlmaterialabtragsanlage variiert werden. Die aufgezeigten Zusammenhänge werden somit als Richtlinien für die Auslegung der Bearbeitungsparameter hinsichtlich einer hohen zeitlichen Materialabtragsrate, einer geringen Rauheit und einer hohen Formtreue eingesetzt.

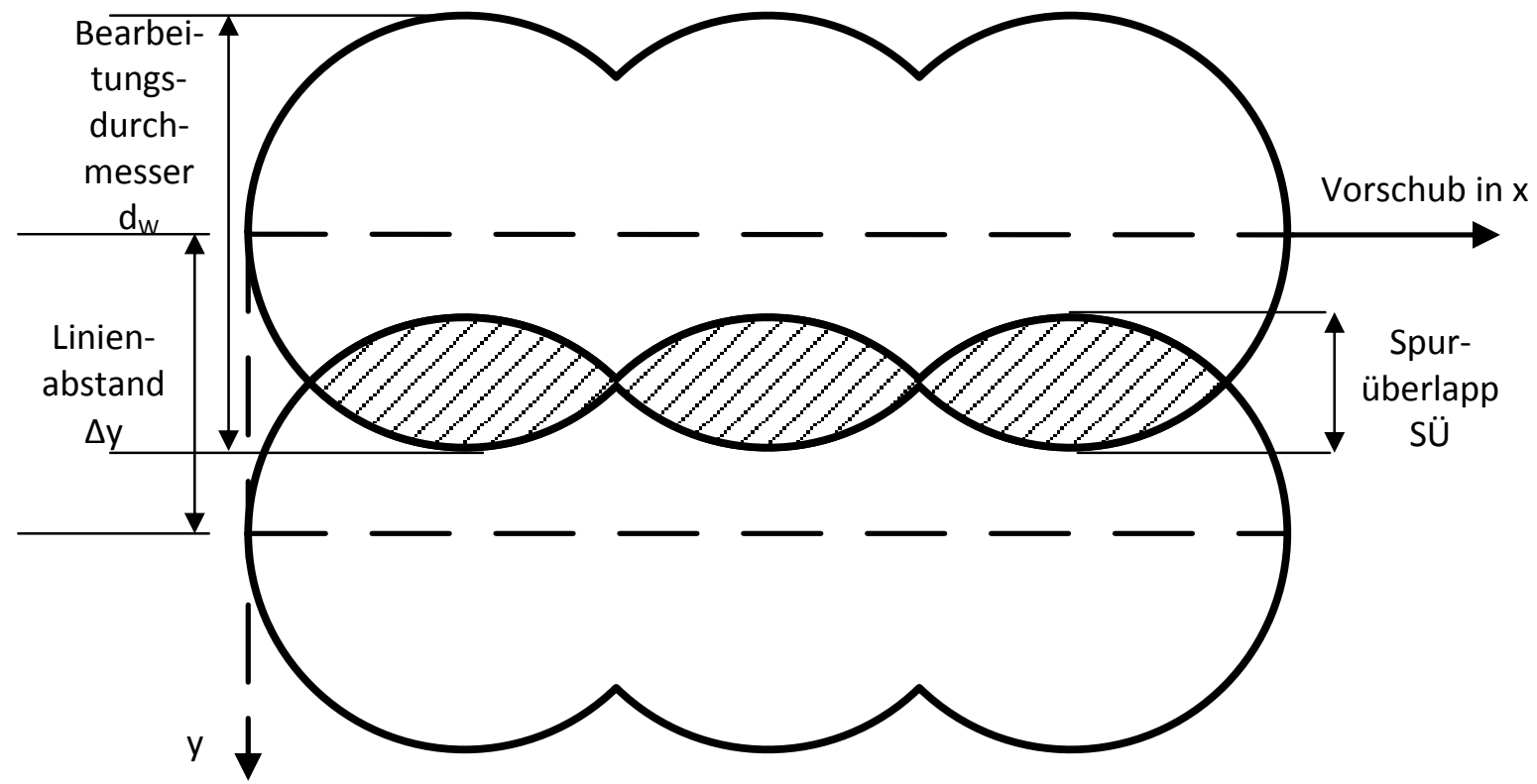

Abb. 19: Schematische Darstellung des Spurüberlapps SÜ. Angelehnt an Kagerer, Irlinger und Lueth (2011) sowie DIN 32540 (Stand August 2012). 


\subsubsection{Fertigung senkrechter Kanäle mithilfe des Trennschleifens}

Wie bereits beschrieben, gilt es, Kanäle respektive Düsen in Siliziumsubstraten mithilfe des Trennschleifverfahrens zu fertigen. Hierbei wird eine Wafersäge verwendet. Die resultierenden Vorteile hierbei sind:

- Fertigung von Mikrokanälen mit steilen Flanken

- Seitenwände und Böden der Mikrokanäle mit geringer Rauhtiefe $\mathrm{R}_{\mathrm{z}}$ von bis zu $0,1 \mu \mathrm{m}$ (Fischer et al., 2008)

Dies bietet zum einen definierte Düsendimensionen im Gegensatz zu einer konisch zulaufenden Form bei einer Fertigung mittels Laserstrahlbearbeitung und zum anderen wird auftretende Reibung im Mikrokanal für die Tropfengenerierung durch die glatten Seitenwände minimiert.

Das Schnittergebnis für das Trennschleifen mittels Wafersäge ist hauptsächlich abhängig von den Materialeigenschaften des verwendeten Sägeblattes und den Schnittparametern (Drehzahl und Vorschub). Möglicherweise auftretende unerwünschte Effekte sind sowohl Chipping als auch ein Wellenschnitt (siehe Abb. 20). Chipping bezeichnet hierbei Materialabplatzungen an den Sägekanten, während ein Wellenschnitt bedeutet, dass die Schnittkerbe nicht senkrecht, sondern wellenförmig verläuft. Daher ist es wichtig, zu Beginn der Prozessbeschreibung den Einfluss der Materialeigenschaften von Sägeblättern und auch von den Schnittparametern zu ermitteln.

Sägeblätter für das Trennschleifen bestehen meist aus einem Stahlkern an dessen äußerem Rand sich in einer Matrix gebunden Schleifkörper befinden. Als Schleifkörper haben sich Diamant oder auch Bornitrid als besonders geeignet gezeigt. Dabei gilt, je kleiner das Korn ist, desto feiner ist der Schnitt, allerdings reduzieren sich damit die Blattstandzeit und der mögliche Vorschub. Als Matrix bewähren sich besonders Metallbindungen (Kupfer oder Nickel) und auch Kunststoffbindungen. Werden harte und spröde Materialien bearbeitet, werden Sägeblätter mit einer Kunststoffbindung eingesetzt. Der Vorteil hierbei ist, dass stumpfe und abgenutzte Schleifkörner freigegeben und neue unbenutzte Körner exponiert werden. Man spricht hierbei auch von einem Selbstschärfeeffekt. Für Materialien, die generell weicher sind, steht vor allem die Blattstandzeit im Vordergrund. Hierbei ist insbesondere die metallische Nickelbindung für die Bearbeitung von Silizium bevorzugt (minitron elektronik, 2014). Werden zudem noch feine Schnitte verlangt, wie bspw. bei der Düsenfabrikation, werden nickelgebundene Blätter mit kleinen Schleifkörnern $(<10 \mu \mathrm{m})$ eingesetzt.

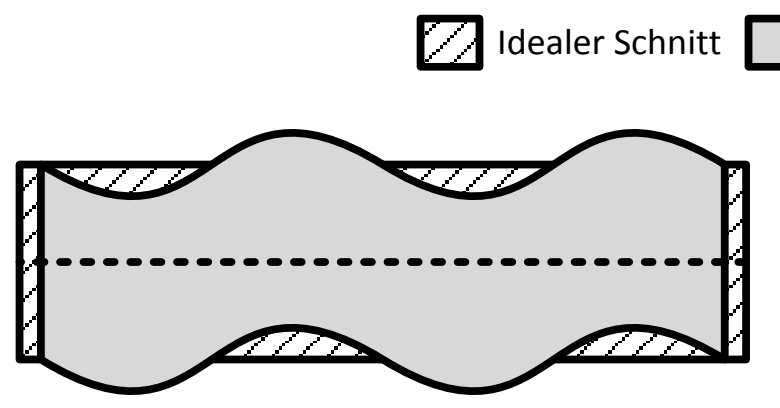

(a)
Auftretender Fehler

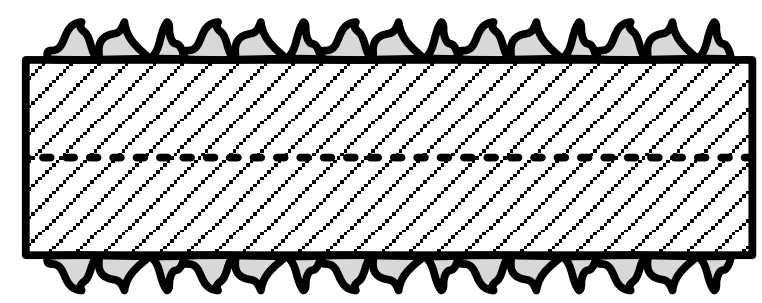

(b)

Abb. 20: Schematische Darstellung möglicher Fehler bei dem Trennschleifprozess. (a) Welligkeit des Schnittes und (b) Chipping (Materialabplatzungen) an den Sägekanten. 
Neben der korrekten Wahl des Sägeblattes ist jedoch auch der Einfluss der Schnittparameter (Drehzahl und Vorschub) auf das Schnittergebnis zu beschreiben. Charakteristisch ist, dass die Welligkeit eines Schnittes mit dem Vorschub steigt. Der Grund hierfür ist, dass das Sägeblatt auf Grund seines Blattüberstandes dem Werkstück seitlich der Schnittkerbe ausweicht, da auf die Schneide hohe Scherkräfte wirken (minitron elektronik, 2014). Ebenfalls steigt mit dem Vorschub auch das Chipping seitlich der Sägekanten / -spuren. Ein hoher Vorschub leitet in das Substrat hohe Kräfte ein, so dass sich Aussprünge weiter ausbreiten. Eine Erhöhung der Spindeldrehzahl verringert das auftretende Chipping. Wird die Drehzahl bei gleichem Vorschub erhöht, bleibt die gesamte Abtragsrate konstant, jedoch verringert sich der jeweilige Materialabtrag pro Umlauf des Blattes. Dies bedeutet, dass die zum Abtragen benötigte Energie sich auf mehrere Umläufe verteilt, so dass im Ergebnis die auftretende Belastung auf das Substrat reduziert wird.

Wafersägen werden hauptsächlich verwendet, um einzelne Dies aus einem Wafer herauszutrennen. Hierbei wird das Werkstück komplett mit dem Sägeblatt durchtrennt. Für die Bearbeitung werden die Substrate auf eine Sägefolie geklebt, auf einem Vakuumteller fixiert und im Anschluss durchtrennt. Die Tiefe des Schnittes ergibt sich hierbei aus einem Abstandsmaß, wie weit entfernt der Schnitt von der Vakuumtelleroberfläche endet. In der Regel wird das Maß so gewählt, dass die Sägefolie zur Hälfte ihrer Materialdicke durchtrennt wird, so dass die durchgetrennten Dies noch an der Sägefolie für eine leichtere Handhabung haften. Da vorliegend jedoch die Tiefe der Kanäle relativ zu der Oberfläche des Siliziumsubstrates zu fertigen ist, ist diese Herangehensweise zu ungenau. Des Weiteren unterliegen in der Mikrotechnik die verwendeten Materialien gewissen Dickenschwankungen, welche verteilt über das Substrat unterschiedlich hoch ausfallen.

Daher ergibt sich die Forderung, dass für jeden zu fertigenden Kanal auf dem Substrat die relative Höhenposition der Oberfläche bekannt sein muss. Hierzu wird ein Verfahren eingeführt, welches die relative Position der Oberfläche bestimmt. An einer Stelle nahe der Sollposition des Mikrokanals wird das Sägeblatt in Mikrometerschritten $(<5 \mu \mathrm{m})$ sukzessive abgesenkt, bis eine Kerbe auf der Oberfläche sichtbar ist. Diese Höhenposition wird als Nulllage der Substratoberfläche definiert. Im Anschluss wird das Sägeblatt an die Startposition des zu fertigenden Mikrokanals versetzt, die gewünschte Tiefe des Kanals in der Wafersäge eingestellt und der Prozess gestartet. Hiermit wird sichergestellt, dass definiert tiefe Mikrokanäle respektive Düsen erstellt werden.

Neben dem Erstellen der Mikrokanäle in definierter Tiefe gilt es auch, Kanäle mit unterschiedlichen Breiten zu fertigen. Die Breite der Düse bestimmt maßgeblich das ausgestoßene Tropfenvolumen bei einem Tropfenerzeuger.

Die Breite des verwendeten Schnittes ist abhängig von der Sägeblattbreite. Auch wenn es viele unterschiedlich breite Sägeblätter zu erwerben gibt, so ist man dennoch auf diese Breiten limitiert. Möchte man stattdessen Kanäle fertigen, welche unabhängig von dem Sägeblatt sind, so gilt es, versetzte Schnitte in einem bestimmten Überlapp zu nutzen. Allerdings ist dabei der Versatz zu beachten. Ein zu großer Versatz der Schnitte voneinander resultiert in einem unebenen Kanalboden mit sichtbaren Rillen. Diese Unebenheit würden den fluidmechanischen Tropfenbildungsprozess nicht stabil gestalten, da das Fluid während seiner Beschleunigung in der Düse diesem Widerstand ausgesetzt ist. Daher ist ein kleiner Versatz zu wählen, um einen ebenen Boden zu garantieren. In vorliegendem Projekt wird daher ein Versatz von höchstens einem Drittel der Sägeblattbreite gewählt. Um einen Kanal mit einem Sä- 
geblatt der Breite $\mathrm{d}$ und einem Versatz $\Delta \mathrm{v}$ zu fertigen, ergibt sich bei einer Anzahl $\mathrm{n}$ versetzter Schnitte eine Kanalbreite b nach (7.13).

$$
b=d+\Delta v \cdot(n-1)
$$

Durch Auflösen von (7.13) nach $\Delta \mathrm{v}$ ergibt sich im Ergebnis der einzustellende Versatz (siehe (7.14)). Die Anzahl der auszuführenden Schnitte $n$ stellt immer eine ungerade Zahl dar. Der Grund hierfür ist, dass den Ausgang hierbei immer ein mittig gelegener Ausgangsschnitt bildet, der als Referenz für die nachfolgenden Schnitte dient. Die weiteren Schnitte werden dann alternierend sowohl rechts als auch links davon gefertigt.

$$
\Delta v=\frac{b-d}{(n-1)}
$$

Beispielsweise werden mit vorgegebener Auslegungsvorschrift für einen geforderten $70 \mu \mathrm{m}$ breiten Mikrokanal und einem Sägeblatt mit einer nominalen Breite von $50 \mu \mathrm{m}$ drei Schnitte benötigt. Hierbei beträgt der Versatz der Schnitte $\Delta \mathrm{v} 10 \mu \mathrm{m}$ nach (7.14). Abb. 21 zeigt hierzu das Vorgehen schematisch auf.

Zusammenfassend ergeben sich die Schritte zu der Fertigung von Mikrokanälen mit definierter Breite und Tiefe wie folgt:

1. Bestimmung der Nulllage der Substratoberfläche mittels sukzessiven Herabsenkens des Sägeblattes, bis eine leichte Kerbe sichtbar ist

2. Anfahren der zentralen Kanalposition und Ausführung eines Ausgangsschnittes

3. Sofern Kanäle breiter als die Sägeblattbreite gefordert sind, alternierende überlappende Schnitte nach (7.13) und (7.14) durchführen

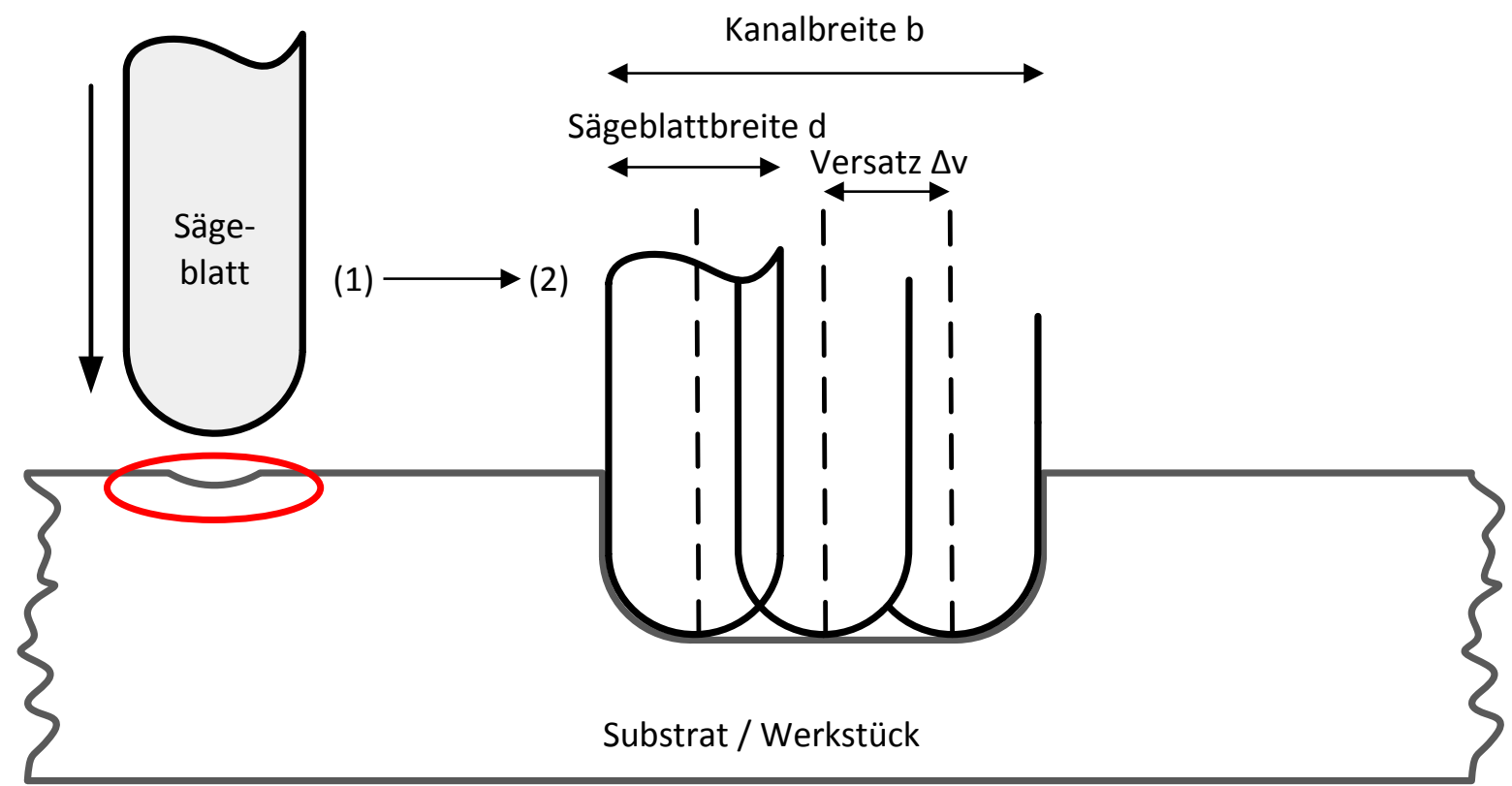

Abb. 21: Schematische Darstellung des Trennschleifprozesses. Der erste Schritt ist die Bestimmung der Nulllage der Substratoberfläche. Im Anschluss wird an die gewünschte Kanalposition gefahren und mittels überlappender Schnitte die geforderte Kanalbreite erzeugt. 


\subsection{Mikromontage von Bauteilen}

Wie bereits beschrieben, sind nicht nur Mikrostrukturierungs-, sondern auch Mikromontageoperationen in der Fertigung von Mikrosystemen notwendig. Bei kommerziell erwerbbaren Mikromontageanlagen stehen hierbei das Handhaben und das Fügen immer kleinerer Bauteile im Vordergrund. Im Gegensatz dazu steht in vorliegender Arbeit das Handhaben und Fügen von planaren Substraten mit Bauteildimensionen im Millimeterbereich, wie bspw. piezoelektrischen Keramiksubstraten, im Fokus der Anwendung. Es werden zusammenfassend folgende Anforderungen an den zu entwickelnden Mikromontageplatz gestellt: Relativpositionierung von zwei Bauteilen, ganzflächiger Auftrag von Klebstoff, kostengünstige Entwicklung mit kleiner Maschinenstellfläche, integrierte Heizmöglichkeit und intuitive Bedienung mit geringen Rüstzeiten.

Ziel ist es daher, in einem Werkzeug alle benötigten Funktionen für die Fügung von zwei Bauteilen an einem Ort zur Verfügung zu stellen, um Um- bzw. Neujustageprozesse zu vermeiden, Prozessfehler zu verhindern und die Maschinenstellfläche zu reduzieren.

\subsubsection{Aufbau des Mikromontageplatzes}

Der Aufbau des Mikromontageplatzes wird sich durch eine hohe Modularität, einer intuitiven Bedienbarkeit, einer geringen Maschinenstellfläche und einer kostengünstigen Entwicklung auszeichnen. Die wesentlichen Komponenten sind hierbei (siehe auch Abb. 22):

- Rahmen inklusive Seitenwände zu der Befestigung der notwendigen Werkzeuge und Bodenplatte zur Fixierung des Mikromontageplatzes auf einem Arbeitstisch

- Montageplattform für die Ausrichtung und Relativpositionierung von Bauteilen inklusive Heizmöglichkeit

- Greif- / Absetzwerkzeug für die Montage eines Bauteils auf dem Bauteil, welches sich auf der Montageplattform befindet

- Verfahrbare Höhenachse mit der die Montageplattform an das Greif- / Absetzwerkzeug grob angenähert werden kann

- Beobachtungseinheit zur Visualisierung von Relativpositionierungen und Bauteilausrichtungen

- Auftragswerkzeug für eine ganzflächige, dünne und homogene Klebstoffschicht auf Bauteilen, welche sich auf der Montageplattform befinden

- Peripherie, wie bspw. Temperaturregler, Stromversorgung oder Computer

Sollen zwei Bauteile exakt zueinander ausgerichtet und auch über eine Klebstoffschicht gefügt werden, wird das abzusetzende Bauteil über das Greifwerkzeug aufgenommen. Das Zielbauteil, welches sich auf der Montageplattform befindet, wird über die Beobachtungseinheit zu dem abzusetzenden Bauteil auf dem Greifwerkzeug ausgerichtet. Hierfür bietet die Montageplattform sowohl zwei translatorische Freiheitsgrade (X- und Y-Richtung) als auch einen rotatorischen Freiheitsgrad ( $\theta$-Richtung). Die Montageplattform ist an einer manuell verfahrbaren Höhenachse montiert. Eine grobe vertikale Annäherung beider Bauteile erfolgt mit die- 
ser Achse, so dass nur ein geringer Spalt zwischen beiden Bauteilen besteht. Dieser Spalt wird über das Greifwerkzeug, welches in seiner vertikalen Lage zum Absetzen des Bauteils verändert werden kann, ausgeglichen. Wird zwischen beiden Bauteilen eine homogene und definierte Klebschicht gefordert, so kann diese mit einem Auftragswerkzeug aufgebracht werden. Ein integriertes Heizelement in der Montageplattform erlaubt zusätzlich das Hinzufügen thermischer Energie, um bspw. den Klebeprozess durch verbesserte Quervernetzung der einzelnen Bestandteile des Klebstoffes während der Aushärtephase zu beschleunigen.

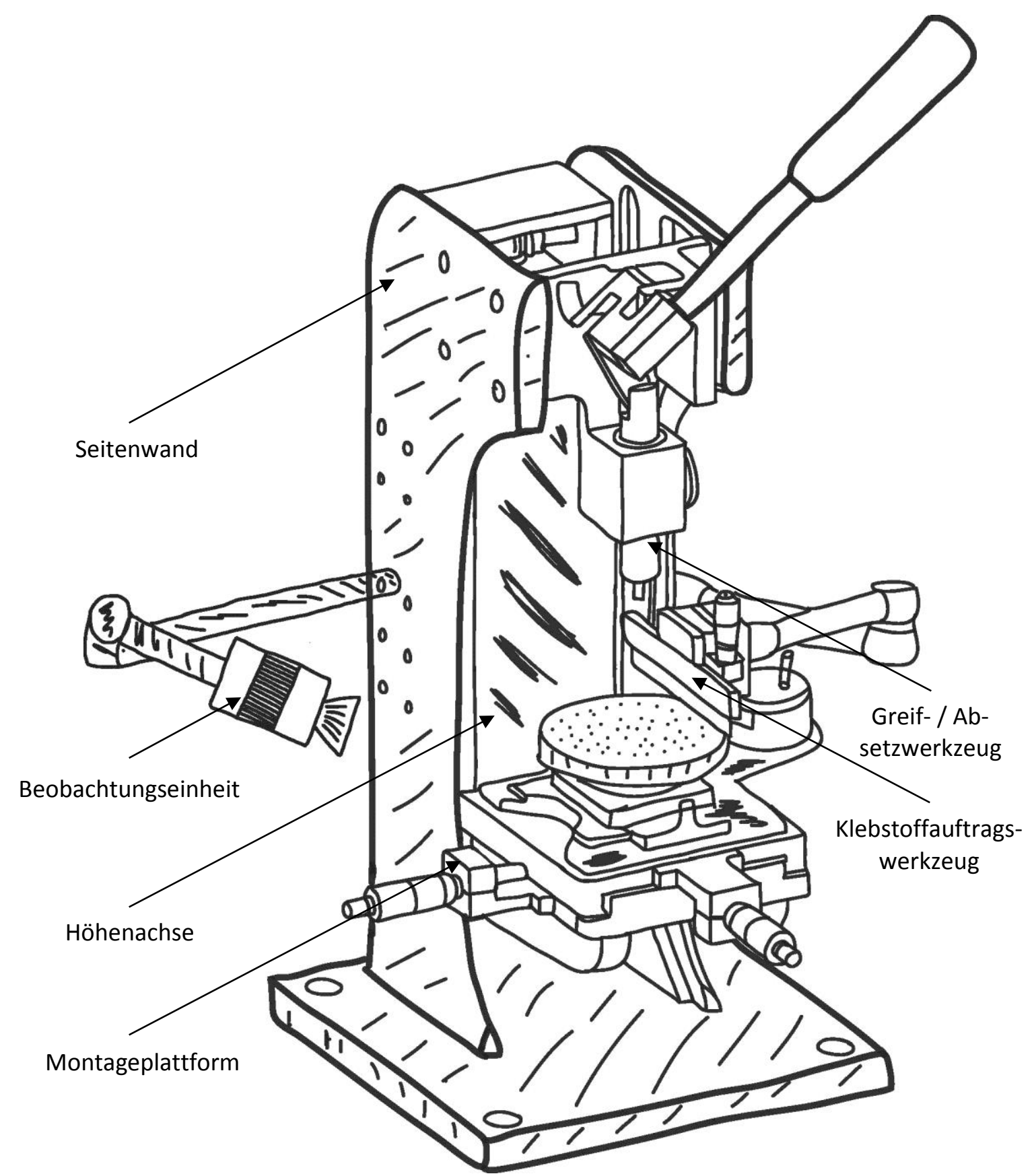

Abb. 22: Schematische Darstellung des Mikromontageplatzes mit seinen wesentlichen Baugruppen, welcher sowohl für Relativpositionierungen als auch für den Klebstoffauftrag verwendet werden kann. Der Mikromontageplatz zeichnet sich durch eine hohe Modularität aus, so dass über Führungen und Gewindebohrungen an den Seitenwänden zusätzliche Werkzeuge angebracht werden können. 
Dabei stellt der Rahmen das größte und massivste Konstruktionselement vorliegenden Mikromontageplatzes dar. Dieser besteht aus zwei Seitenwänden, einer Bodenplatte und einer Rückwand.

Die Bodenplatte stellt die Basis für den Gesamtaufbau dar. An dieser werden die Seitenwände angebracht. Diese gilt es, massiv auszuführen, so dass der Schwerpunkt des Gesamtsystems herabgesetzt wird und der Mikromontageplatz Stabilität erlangt. Zusätzlich werden in der Bodenplatte Durchgangslöcher eingearbeitet, über die der Mikromontageplatz an einem Arbeitstisch befestigt wird und hierdurch zusätzlich Stabilität gewinnt.

An der Bodenplatte werden die beiden Seitenwände über Schraubverbindungen montiert. Der Grundgedanke bei der Entwicklung der Seitenwände ist es nicht nur, hierdurch die Stabilität des Mikromontageplatzes, sondern auch seine hohe Modularität zu gewährleisten. Im Ergebnis bedeutet dies, dass die Seitenwände mit diversen Gewindebohrungen und Führungsschienen versehen werden, an denen Werkzeuge befestigt werden können. In vorliegender Grundkonfiguration des Montagesystems (siehe Abb. 22) werden sowohl die Beobachtungseinheit als auch das Klebstoffauftragswerkzeug über bewegliche Haltearme an den Seitenwänden angebracht. Die über die Seitenwände verteilten Gewindebohrungen und Führungsschienen erlauben hierbei die Integration einer Vielzahl verschiedenster Werkzeuge an beliebigen Positionen, wie bspw. Lötkolben, welche direkt in den Montageprozess von Mikrosystemen eingebunden werden. Sofern die Werkzeuge nicht benötigt werden, können entweder die Haltearme von den Seitenwänden abgenommen oder auch seitlich eingeklappt werden. Abb. 22 zeigt, dass die Form der Seitenwände näherungsweise einem C-Bogen entspricht. Der Grund hierfür ist die leichtere Zugänglichkeit mit Werkzeugen von der Seite aus, wodurch die Bedienung des Mikromontageplatzes zusätzlich erleichtert wird.

Ein weiteres zentrales Element des Mikromontageplatzes ist eine verfahrbare Höhenachse, welche an der Rückwand befestigt wird. Hier ist die Montageplattform mit integrierter Heizmöglichkeit angebracht. Durch Verfahren dieser Achse wird die Plattform mit aufgelegtem Bauteil an das Greif- / Absetzwerkzeug angenähert. Der noch verbleibende Abstand beider Bauteile wird mit dem Greif- / Absetzwerkzeug ausgeglichen. Es gilt für die Höhenachse einen ausreichenden Verfahrweg zu gewähren, so dass neben der Annäherung an das Greif- / Absetzwerkzeug auch auf der anderen Seite ein ausreichend hoher Abstand hiervon gewährleistet werden kann, um Bauteile auf der Montageplattform leichter aufzulegen und auch zu entnehmen. Zum Heben und Senken der Achse wird eine Handkurbel eingesetzt. Das Gesamtsystem zeichnet sich durch einen äußerst platzsparenden Aufbau aus.

Die Beobachtungseinheiten für die Montageoperationen werden in der Grundkonfiguration des Mikromontageplatzes Videomikroskope darstellen, welche kostengünstig zu erwerben sind. Da die vorliegende Entwicklung nicht das Ziel verfolgt, Bauteile im Mikrometerbereich handzuhaben, ist die Abbildungsvergrößerung von Videomikroskopen ausreichend und es erfolgt eine an die Randbedingungen angepasste und kostengünstige Entwicklung.

Nachfolgend wird der eigene Ansatz bei der konstruktiven Umsetzung für die Realisierung der beiden geforderten Mikromontageoperationen, Absetzen und Ausrichten mit Relativpositionierung zweier Bauteile sowie Klebstoffauftrag, beschrieben. Hierbei wird ersichtlich, dass vorliegendes System intuitiv bedienbar und kostengünstig ist. Es werden alle benötigten Werkzeuge für die Fügung von zwei Bauteilen an einem Ort zur Verfügung gestellt. Hierdurch werden Um- bzw. Neujustageprozesse verhindert, Prozessfehler reduziert und die Maschinenstellfläche verringert. 


\subsubsection{Ausrichten und Absetzen von zwei Bauteilen}

Mit vorliegendem Mikromontageplatz wird die Fügung von zwei Bauteilen über eine Klebstoffschicht ermöglicht. Hierzu befindet sich das Zielbauteil auf der Montageplattform; das abzusetzende Bauteil wird über das Greif- / Absetzwerkzeug hierauf montiert. Damit beide Bauteile zueinander exakt ausgerichtet werden können, bedarf es drei Freiheitsgrade im System. Zum einen ist dies die X- und Y-Richtung, mit der das Zielbauteil auf der Montageplattform relativ zu dem abzusetzenden Bauteil ausgerichtet wird. Zum anderen ist eine rotatorische Ausrichtung erforderlich ( $\theta$-Richtung), um die Parallelität beider Bauteile zu gewähren.

Abb. 23 zeigt hierzu schematisch die Montageplattform, mit der das Zielbauteil zu dem abzusetzenden Bauteil ausgerichtet wird. Dabei erfolgt die Ausrichtung sowohl mit einem Kreuztisch für die X- und Y-Richtung als auch mit einem Drehtisch für die $\theta$-Richtung. Die Plattform wird über Gabelzinken direkt an die verfahrbare Höhenachse befestigt. Der Einsatz eines Kreuztisches bietet eine hohe Wiederholgenauigkeit der Positionierung und auf der anderen Seite eine stufenlose Bewegung in beide Richtungen. Auf dem Kreuztisch wird wiederum der Drehtisch befestigt. Die Drehachse muss senkrecht zu der XY-Ebene ausgerichtet werden. Für die Aktuierung des Kreuztisches werden zwei Mikrometerschrauben eingesetzt. Die Aktuierung des Drehtisches erfolgt über einen Zahnriemen in Verbindung mit einer Handkurbel.

Das Zielbauteil wird auf dem Drehteller, welcher auf dem Drehtisch montiert ist, mittels Vakuum fixiert. Der Durchmesser des Drehtellers beträgt in der Grundkonfiguration $100 \mathrm{~mm}$, so dass auch die Möglichkeit zur Fixierung von standardisierten 4 Zoll Wafern besteht. Für die Vakuumansaugung des Bauteils werden konzentrisch und gleichmäßig angeordnete Sauglöcher in den Drehteller eingelassen.

Auf der Unterseite des Drehtellers wird eine Heizspirale angebracht, so dass der Montagevorgang mit Temperatur unterstützt werden kann. Damit die Wärme direkt an das Zielbauteil

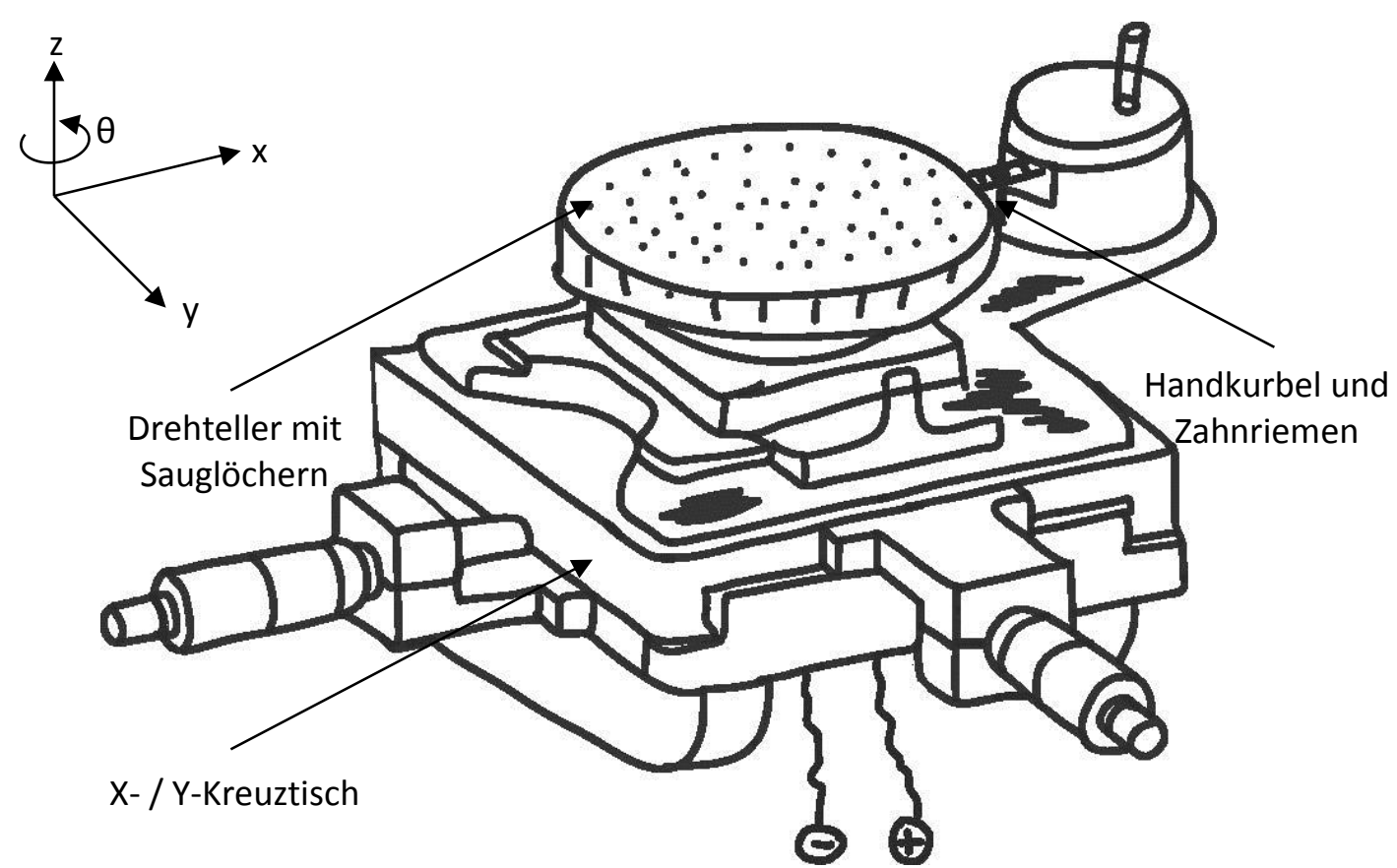

Abb. 23: Schematische Darstellung der Montageplattform. Über einen Zahnriemen und eine Handkurbel erfolgt die Drehung des Tisches, welcher sich auf einem X- / Y-Kreuztisch befindet. Mit Hilfe von Mikrometerschrauben wird der Kreuztisch bewegt. Zusätzlich ist die elektrische Anschlussleitung für die integrierte Heizspirale abgebildet. 
gelangt, ist für den Drehteller ein Material mit hohen Wärmeleitkoeffizienten zu wählen und die Heizspirale nahe der Oberfläche des Tisches zu positionieren. Gleichzeitig gilt es jedoch, auch den Wärmeverlust auf der Unterseite des Drehtellers zu minimieren und ihn somit auf die Oberfläche zu konzentrieren. Hierzu gilt es, eine entsprechende Wärmeisolierung unterhalb des Drehtellers anzubringen. Darüber hinaus erfolgt nur eine verringerte Erwärmung der umliegenden Bestandteile der Plattform, so dass ein minimiertes Verletzungsrisiko für den Operator besteht.

Für das Absetzen des Bauteils ist ein Mechanismus zu wählen, welcher zum einen ein genaues Positionieren über eine stufenlose Absetzbewegung erlaubt und zum anderen eine komfortable und intuitive Bedienung mit Feingefühl ermöglicht. Hierfür eignen sich zentrische Schubkurbelgetriebe, welche eine Drehbewegung in eine Schubbewegung umsetzen (siehe Abb. 24 (a)). Mit Hilfe eines Hebels wird ein oberer Kniehebel nach unten gedrückt. Der Kniehebel selbst ist über einen Pleuel mit einem Kolben verbunden, an dem das Greifwerkzeug angebracht ist. Schubkurbelgetriebe werden nach Hölz, Mollenhauer und Tschöke (2011) über den Kurbelradius $r_{\text {Kurb }}$, der Pleuellänge 1, dem Kolbenweg $\mathbf{x}_{\text {Kolb }}$, dem Kurbelwinkel $\theta_{\text {Kurb }}$ und dem Stangenwinkel $\beta$ beschrieben (siehe Abb. 24 (b)). Ein entscheidender Vorteil von Schubkurbelgetrieben ist ihre Eigenschaft, dass bei Verringerung des Kurbelwinkels $\theta_{\text {Kurb }}$ gleichzeitig auch der Kolbenweg x reduziert wird. Für den Absetzvorgang bedeutet dies, dass zu Beginn die Absetzgeschwindigkeit bei großen Kurbelwinkeln vergleichbar groß ist und zunehmend geringer wird, obwohl die Bewegung am Hebel mit der Hand konstant gehalten wird. Hierdurch wird ein kontrolliertes Absetzen von Bauteilen über das Greif- / Absetzwerkzeug in vertikaler Richtung auf Bauteile, welche sich auf der Montageplattform befinden, mit Feingefühl möglich.
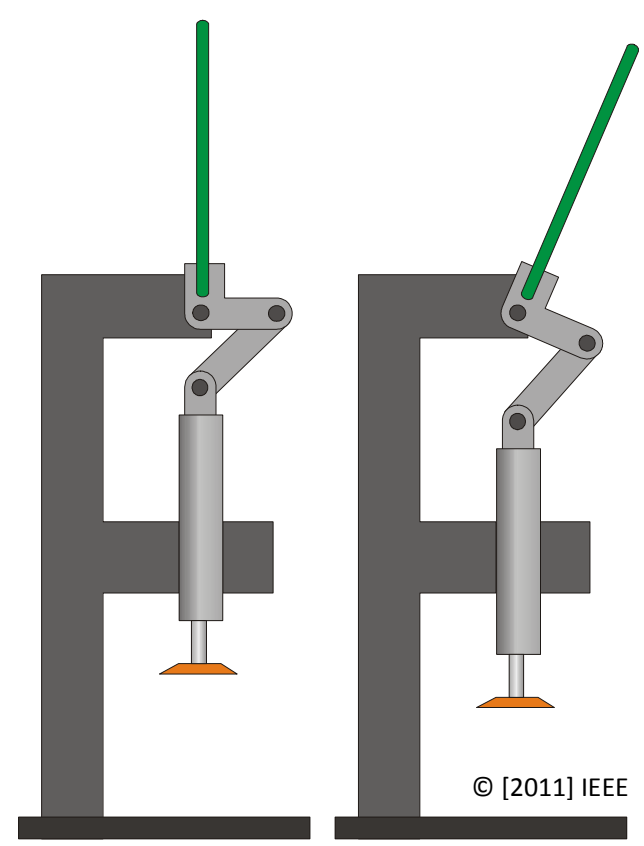

(a)

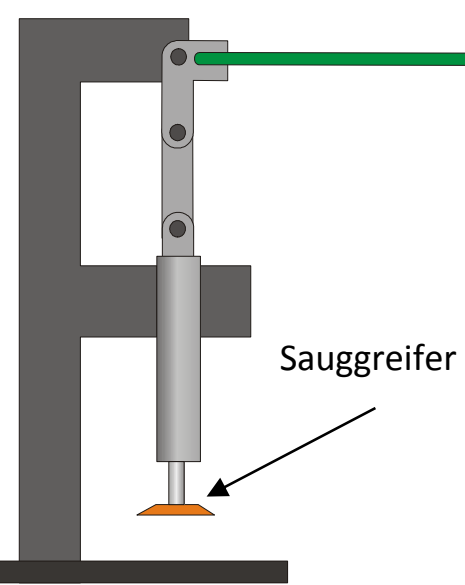

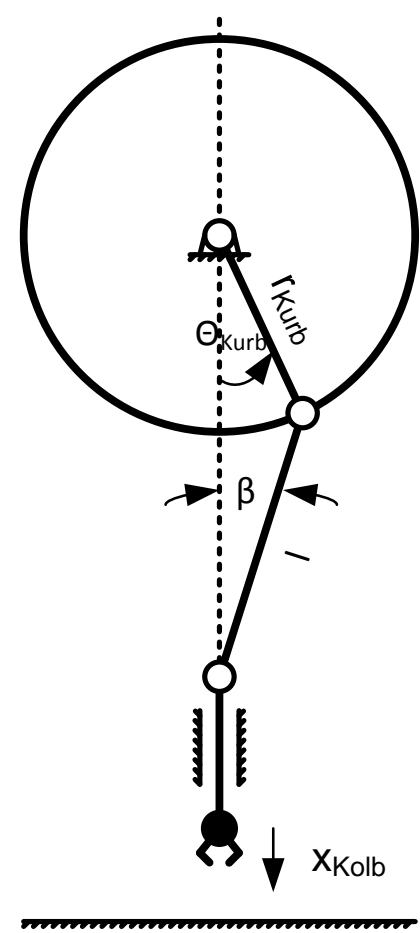

(b)

Abb. 24: Kinematische Umsetzung des Absetzvorganges eines Bauteils. (a) Mit einer Drehbewegung wird der Kolben inklusive Greif- / Absetzwerkzeug nach unten in Richtung der Montageplattform bewegt; entnommen aus Kagerer et al. (2011). (b) Resultierendes Getriebeschema der eingesetzten kinematischen Anordnung mit Kurbelradius $r_{\text {Kurb }}$, Pleuellänge 1, Kolbenweg $\mathrm{x}_{\text {Kolb }}$, Kurbelwinkel $\theta_{\text {Kurb }}$ und Stangenwinkel $\beta$. 
Der zurückgelegte Kolbenweg $\mathrm{x}_{\text {Kolb }}$ wird nach Hagedorn, Thonfeld und Rankers (2009) sowie Hölz, Mollenhauer und Tschöke (2011) in (7.15) angegeben.

$$
x_{\text {Kolb }}=r_{\text {Kurb }} \cdot\left(1-\cos \left(\theta_{\text {Kurb }}\right)\right)+l \cdot(1-\cos (\beta))
$$

Der Stangenwinkel $\beta$ ist hierbei nicht von Bedeutung, da die Abhängigkeit des Kolbenweges $\mathrm{x}_{\text {Kolb }} \mathrm{zu}$ dem Kurbelwinkel $\theta_{\text {Kurb }}$ im Vordergrund des Interesses steht. Mit Hilfe des Sinussatzes wird der Stangenwinkel $\beta$ mit dem Kurbelwinkel $\theta_{\text {Kurb }}$ in (7.16) beschrieben. Zugleich wird der Faktor $\lambda_{\text {Schub }}$ hinzugefügt, welcher das Schubstangenverhältnis beschreibt. Hagedorn, Thonfeld und Rankers (2009) beschreiben, je größer das Schubstangenverhältnis, respektive je kürzer der Pleuel ist, desto ungleichförmiger wird die Gleitsteinbewegung am Kolben.

$$
\begin{aligned}
& \sin (\beta)=\frac{r_{\text {Kurb }}}{l} \cdot \sin \left(\theta_{\text {Kurb }}\right)=\lambda_{\text {Schub }} \cdot \sin \left(\theta_{\text {Kurb }}\right) \\
& \cos (\beta)=\sqrt{1-\sin ^{2}(\beta)}=\sqrt{1-\lambda_{\text {Schub }}^{2} \cdot \sin ^{2}\left(\theta_{\text {Kurb }}\right)}
\end{aligned}
$$

Durch Einsetzen von (7.16) in (7.15) erfolgt in (7.17) die Beschreibung des Kolbenweges $\mathrm{x}_{\text {Kolb }}$ in Abhängigkeit des Kurbelwinkels $\theta_{\text {Kurb }}$, des Kurbelradius $\mathrm{r}_{\text {Kurb }}$ und der Pleuellänge 1 .

$$
x_{\text {Kolb }}=r_{\text {Kurb }} \cdot\left(1-\cos \left(\theta_{\text {Kurb }}\right)\right)+l \cdot\left(1-\sqrt{1-\lambda_{\text {Schub }}^{2} \cdot \sin ^{2}\left(\theta_{\text {Kurb }}\right)}\right)
$$

Abb. 25 verdeutlicht hierbei den Zusammenhang zwischen Kolbenweg $\mathrm{x}_{\text {Kolb }}$ und Kurbelwinkel $\theta_{\text {Kurb. }}$ Es ist erkennbar, dass trotz einer gleichbleibenden Handbewegung am Hebel die Kurve hin zu kleinen Kurbelwinkeln abflacht. Als Ergebnis wird hieraus gezogen, dass das manuelle Absetzen in einem Kurbelwinkelbereich kleiner $20^{\circ}$ erfolgen wird. Ein Überschreiten eines Kurbelwinkels von $0^{\circ}$ würde aufgrund der kinematischen Gegebenheiten zu einer erneuten Aufwärtsbewegung des Kolbens führen (siehe Abb. 24 (b)). Um einer versehentlichen Weiterdrehung des Hebels entgegenzuwirken, muss eine konstruktive Maßnahme vorgesehen werden. Der Hubkolben wird mit Hilfe eines Gleitlagers am Mikromontageplatz geführt.

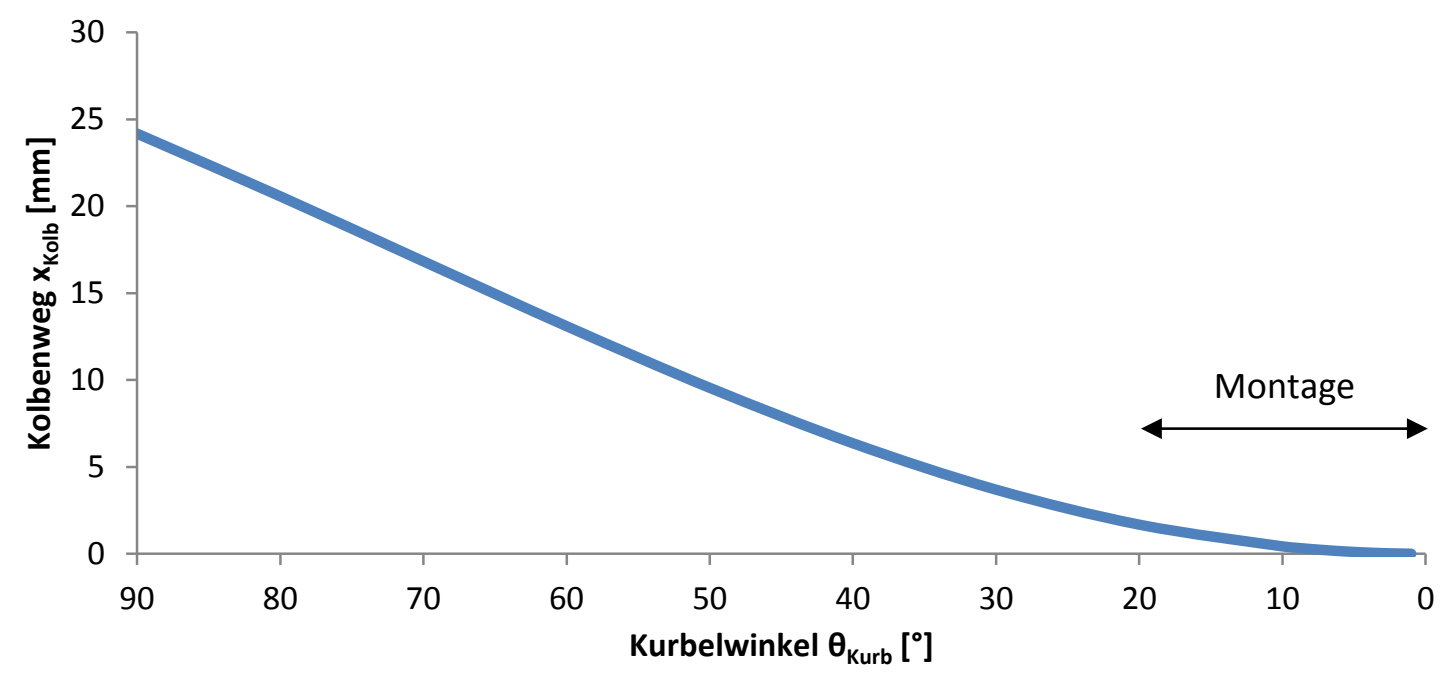

Abb. 25: Darstellung des Zusammenhanges zwischen Kolbenweg $\mathrm{x}_{\text {Kolb }}$ und Kurbelwinkel $\theta_{\text {Kurb }} \mathrm{Zu}$ Beginn des Absetzvorganges herrscht ein lineares Verhalten. Hin zu kleineren Kurbelwinkeln flacht die Kurve ab. In diesem Bereich erfolgt die Mikromontage. In vorliegender Bewegungskurve beträgt das Schubstangenverhältnis $\lambda_{\text {Schub }}=0,4\left(\mathrm{r}_{\text {Kurb }}=20 \mathrm{~mm}\right.$ und $\left.\mathrm{l}=50 \mathrm{~mm}\right)$. 
Nach der Beschreibung der gewählten Kinematik für den Absetzvorgang ist das Greifwerkzeug zu bestimmen. Eine zentrale Anforderung an den Mikromontageplatz ist seine kostengünstige Entwicklung bzw. Herstellung. Um diese Aufgabe auch bzgl. des Greifwerkzeuges zu erfüllen, werden standardisierte Vakuumsauggreifer in vorliegendem Mikromontageplatz eingesetzt. Neben einer kostengünstigen Anschaffung zeichnen sich diese auch durch eine mögliche sanfte Handhabung empfindlicher Bauteile, einer einfachen Systemintegration und einem geringen Gewicht aus. Somit erfolgt auch eine an die Randbedingungen angepasste Auswahl des Greifwerkzeuges.

Vakuumsauggreifer werden hinsichtlich ihrer Form und ihres Werkstoffes unterschieden. Standardisierte runde Sauger eignen sich für flache und leicht gewellte Werkstückoberflächen; ovale Sauger für längliche Werkstücke und Faltenbalgsauger für schiefe Flächen. Im Ergebnis bedeutet dies, dass für planare Substrate standardisierte runde Sauggreifer ausreichend sind. Als Werkstoff wird sowohl Nitrilkautschuk als auch Fluorkautschuk zum Einsatz kommen. Beide Werkstoffe sind gegenüber üblichen Reinigungsmitteln, wie Isopropanol, Wasser und auch Laugen, sehr beständig. Somit kann auch während der Montage eine Reinigung erfolgen. Fluorkautschuk zeichnet sich zusätzlich noch durch eine hohe Temperaturbeständigkeit von bis zu $200{ }^{\circ} \mathrm{C}$ aus (Festo, 2014). Für die Auslegung des Sauggreifers muss neben der Werkstoffauswahl und der Form auch die Abreißkraft $\mathrm{F}_{\mathrm{A}}$, welche sich aus der Haltekraft $\mathrm{F}_{\mathrm{H}}$ und der Anzahl $\mathrm{n}$ an Sauggreifern für die Handhabung eines Bauteils zusammensetzt, mitberücksichtigt werden. (7.18) zeigt hierzu die Berechnung der Abreißkraft $F_{A}$ auf.

$$
F_{A}=\frac{F_{H}}{n}
$$

Die Berechnung der Haltekraft $\mathrm{F}_{\mathrm{H}}$ erfolgt in (7.19) für eine horizontale Greiferlage und eine vertikale Bewegungsrichtung. Hierzu muss neben der Masse des Bauteils m, der Erdbeschleunigung $\mathrm{g}$ auch die Beschleunigung in Bewegungsrichtung a mitberücksichtigt werden. Da eine reine vertikale Bewegung mit der Handkurbel durchgeführt wird, ist diese vernachlässigbar. Ergänzt wird (7.19) mit einem Sicherheitsfaktor S.

$$
F_{H}=m \cdot(g+a) \cdot S
$$

Wird vorliegend bspw. ein standardisierter 4 Zoll Wafer mit einer Dicke von $500 \mu \mathrm{m}$ aufgenommen, ergibt sich eine Haltekraft $\mathrm{F}_{\mathrm{H}}$ von $\sim 0,2 \mathrm{~N}$ bei Silizium $\left(\rho=2,33 \mathrm{~g} / \mathrm{cm}^{3}\right)$ und von $\sim 0,6 \mathrm{~N}$ bei einer Piezokeramik $\left(\rho=8 \mathrm{~g} / \mathrm{cm}^{3}\right)$ bei einem Sicherheitsfaktor $\mathrm{S}$ von 2 . Die vorgestellte Grundkonfiguration des Mikromontageplatzes beinhaltet einen Sauggreifer, so dass die Abreißkraft der Haltekraft entspricht. Anhand dieser Daten kann sowohl der entsprechende Sauggreifer als auch die Vakuumquelle ausgewählt werden. Die resultierende Ansaugkraft $F_{S}$ berechnet sich nach (7.20) aus der Druckdifferenz $\Delta \mathrm{p}$ von Umgebungsdruck und dem erzeugten Unterdruck sowie aus der Fläche A des Druckunterschiedes.

$$
F_{S}=\Delta p \cdot A
$$

Um ein sicheres Handhaben von Bauteilen sicherzustellen, hat die resultierende Ansaugkraft $\mathrm{F}_{\mathrm{S}}$ größer zu sein als die Abreißkraft $\mathrm{F}_{\mathrm{A}}$ bzw. vorliegend als die Haltekraft $\mathrm{F}_{\mathrm{H}}$. Da in der Mikrosystemtechnik und insbesondere im Umgang mit planaren Substraten nur geringe Massen zu handhaben sind, sind auch einfache und kostengünstig erwerbbare Vakuumquellen, wie bspw. Vakuumpumpen oder auch Venturidüsen, für diese Randbedingung ausreichend. 


\subsubsection{Definierter Klebstoffauftrag auf planare Bauteile}

Eine weitere Aufgabe bei der Entwicklung des Mikromontageplatzes ist es, diesen mit einem Werkzeug für den homogenen und ganzflächigen Klebstoffauftrag auf Substraten zu erweitern. Des Weiteren wird eine kurze Rüstzeit für die Inbetriebnahme des Auftragswerkzeuges gefordert, um in einer kurzen Zeit Funktionsmodelle mit unterschiedlichen Klebschichtdicken für Experimente zur Verfügung zu stellen.

Eine mögliche Anwendung hierbei ist bspw. die Verklebung piezoelektrischer Wandler mit Glasmembranen, um einen bimorphen piezoelektrischen Aktor zu formen und hinsichtlich seiner Biegung zu optimieren. Aus Kagerer et al. (2013b) geht hervor, dass mit steigender Klebschichtdicke die Biegesteifigkeit eines bimorphen Aktors steigt. Hiermit verbunden ist gleichzeitig die Abnahme seiner Biegung, da die Biegesteifigkeit nach Pfeifer (1982) in indirektem proportionalen Zusammenhang zu der Biegung steht. Dies bedeutet im Ergebnis, je geringer die Klebschichtdicke eines Klebstoffes ist, desto geringer ist auch die Biegesteifigkeit und desto höher fällt die Biegung des piezoelektrischen Aktors bei identischem Energieeintrag aus. Es wird ersichtlich, dass die Klebstoffschicht so gering wie möglich gehalten werden muss, um einen energieeffizienten Betrieb des Aktors zu gewährleisten.

Um Klebstoffe auf Substrate aufzutragen, sind in Kapitel 2 verschiedene in Mikromontageanlagen integrierte Verfahren vorgestellt worden. Die Gemeinsamkeit dieser Verfahren ist, dass kein homogener und ganzflächiger Klebstoffauftrag möglich ist. Des Weiteren sind unterschiedliche Schichtdicken nicht in kurzer Zeit und meist nicht ohne weiteren Materialaufwand erzielbar. Um diesen Defiziten für eine Kleinserienfertigung in einer Forschungsumgebung, in der unterschiedliche Klebstoffe und Schichtdicken Verwendung finden, entgegenzuwirken, wird folgender eigener Ansatz entwickelt:

Es wird ein Klebstoffauftragswerkzeug entworfen, welches über einen Haltearm direkt an einer der Seitenwände des Mikromontageplatzes angebracht werden kann. Hierbei ergibt sich der bereits genannte Vorteil, dass das Werkzeug seitlich eingeklappt werden kann, sofern dieses nicht benötigt wird. Diese Funktion bietet für weitere Montageoperationen mehr Arbeitsplatz. Das Auftragswerkzeug wird im Wesentlichen aus folgenden Bauteilen bestehen:

- Rakelklinge für die Verteilung des Klebstoffes auf dem Substrat

- Rakelaufnahme für die Fixierung der Rakelklinge

- Linearachse mit Mikrometerstellschraube für die Einstellung der Klebschichtdicke

Das Arbeitsprinzip wird dabei in Teilen der Flexodrucktechnik mit Tauchwalzendruckwerk ähneln. Hierbei wird die Druckfarbe über eine rotierende Walze der Druckform übertragen. Überschüssige Farbe an der Walze wird mit Hilfe einer ortsfesten Rakel in einem festen Abstand zu der Walze abgestreift. Hiermit verbleiben definierte, dünne und ganzflächige Farbschichten auf der Walze.

Der Unterschied zu der Flexodrucktechnik in vorliegender Arbeit ist, dass statt einer rotatorischen Bewegung der Walze eine translatorische Bewegung der Montageplattform unter der Rakelklinge ausgeführt wird. Die Rakel wird in einer Rakelaufnahme mit einer Klemme befestigt. Der Austausch der Rakelklinge wird durch Lösen einer zentralen Schraube ermöglicht. Die Ausrichtung der Klemme erfolgt zusätzlich durch zwei Stifte mit spielfreien Über- 
gangspassungen. Die Spielfreiheit ist an dieser Stelle von entscheidender Bedeutung, da sonst die Gefahr besteht, die Klinge bei ihrem Einbau zu verdrehen und den Rakelprozess im Ergebnis negativ zu beeinflussen. Die Rakelaufnahme wird wiederum an einer Linearachse mit Mikrometerstellschrauben montiert, über die der Abstand von Rakelklinge zu Substrat eingestellt wird. Der Abstand wird der geforderten Schichtdicke entsprechen. Abb. 26 zeigt das integrierte Auftragswerkzeug für Klebstoff für den Mikromontageplatz.

Gilt es, eine Klebstoffschicht zu erstellen, so wird diese als erstes auf einem Substrat aufgebracht und grob verteilt. Wichtig an dieser Stelle ist, dass sich ausreichend Klebstoff über der gesamten Auftragsfläche und vor allem in ausreichender Dicke auf dem Substrat befindet. Im Anschluss wird durch Abstreichen mit der Rakel der Klebstoff gleichmäßig verteilt. Hierfür werden die Mikrometerstellschrauben der Montageplattform in den Prozessablauf hinzugezogen. Das Substrat wird mittels Vakuum auf der Plattform fixiert. Das Auftragswerkzeug wird mit Hilfe des Haltearms ortsfest oberhalb des Substrates positioniert. Die gewünschte Schichtdicke wird als Abstandsmaß zwischen der Oberfläche des Substrates und der Unterseite der Rakelklinge mit Hilfe der Linearachse und ihrer Mikrometerstellschraube eingestellt. Die Voraussetzung hierzu ist, dass zu Beginn zwischen Rakelunterkante und Substrat kein Abstand besteht und die Rakel parallel zu der Substratoberfläche ausgerichtet ist. Durch das Öffnen der Rakelaufnahme mit der zentralen Schraube gleitet die Rakel auf das Substrat herab und richtet sich schließlich parallel zu der Substratoberfläche aus. Ein eventueller Winkelversatz wird hierdurch ausgeglichen. Im Anschluss wird die Montageplattform inklusive fixiertem Substrat unter der ortsfesten Rakelklinge durch das Verfahren der X-Achse (siehe Abb. 26) mit der Mikrometerstellschraube bewegt. Der Klebstoff verteilt sich homogen, ganzflächig und in definierter Dicke auf dem Substrat.

Die resultierende Auftragsfläche des Klebstoffes hängt dabei sowohl von dem Verfahrweg der X-Achse bzw. Mikrometerstellschraube als auch von der Breite der Rakel ab. Daher stellt vorliegender Ansatz für den Klebstoffauftrag ein rein mechanisches Arbeitsprinzip dar.

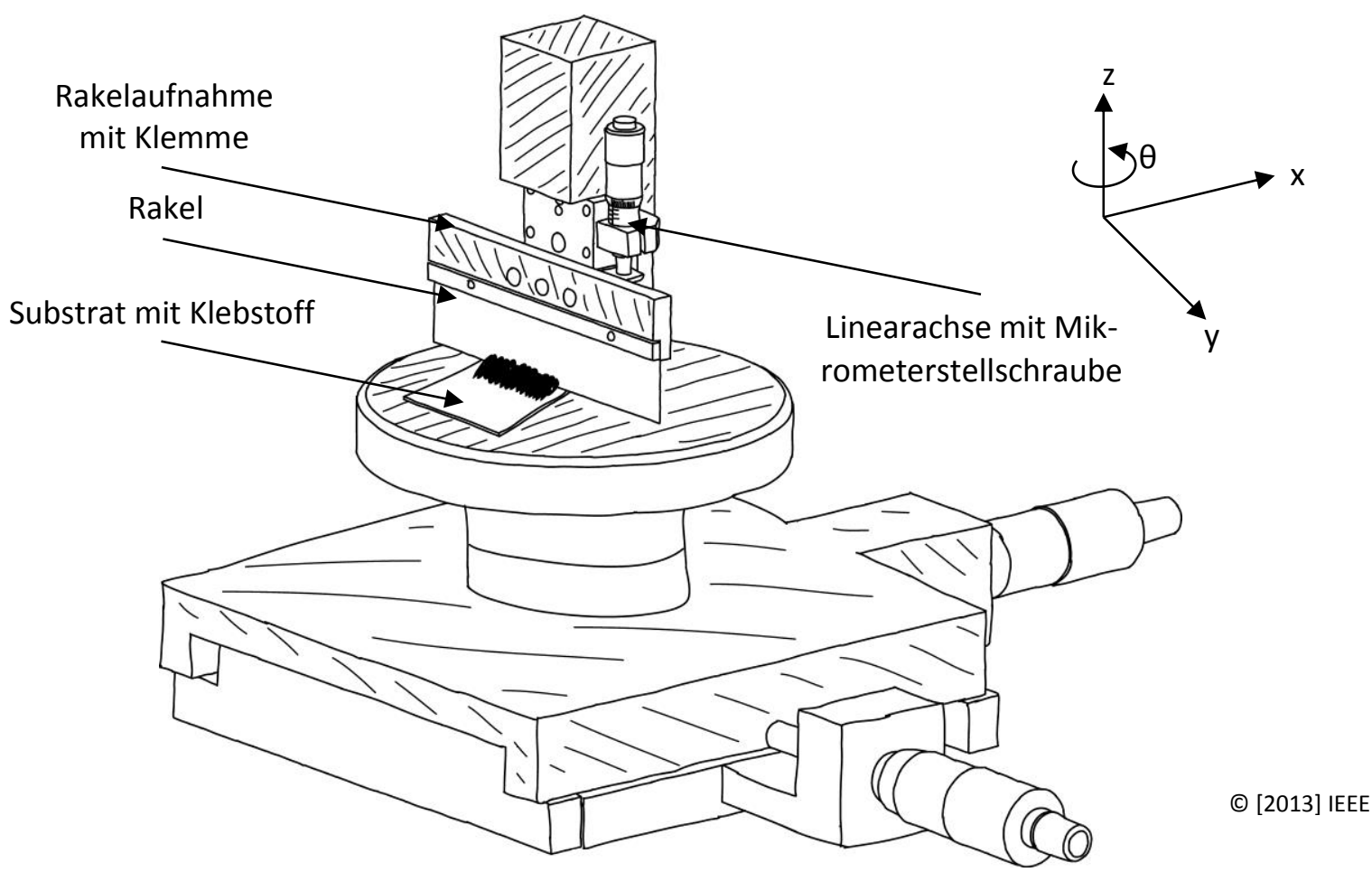

Abb. 26: Schematische Darstellung des Klebstoffauftragswerkzeuges mit Rakelklinge, Rakelaufnahme, Linearachse und Mikrometerstellschraube. Entnommen aus Kagerer et al. (2013b). 
Einen entscheidenden Einfluss auf die Güte des Auftragsergebnisses besitzt die Rakel. Bei der Auswahl ist auf deren Kante und ihre Materialeigenschaften zu achten. Ziel ist es, eine Haftung von Klebstoff an der Rakelkante zu vermeiden, um den Klebschichtauftrag hinsichtlich der Homogenität und der Dicke der aufgebrachten Schicht nicht negativ zu beeinflussen. Für die Haftung von Klebstoff an der Rakelkante ist mitunter ihre Geometrie entscheidend. Je schärfer die Rakelkante bzw. je geringer ihre Querschnittsfläche ist, desto weniger Klebstoff kann an dieser Stelle haften. Hierdurch wird das Abgleiten der Rakel erleichtert und die Scherbeanspruchung des Klebstoffes reduziert sich. Eine weitere Anforderung an die Rakelkante ist, dass diese glatt und nicht wellig ist. Hierdurch wird eine homogene und gleichmäßige Klebstoffschicht aufgetragen. Einen weiteren Einflussfaktor auf das Auftragsergebnis stellt die Biegesteifigkeit der Rakel dar. Sowohl die Adhäsion als auch die Rheologie des Klebstoffes resultieren in einer Kraft, welche mindestens aufzuwenden ist, um den Klebstoff zu verformen bzw. vor der Rakel hinwegzuschieben. Hierdurch wird gleichzeitig eine Kraft auf die Rakel ausgeübt, welche zu einer Biegung führt. Ist die Biegesteifigkeit der Rakel zu gering, wird diese während des Prozesses nach hinten gebogen, was wiederum zu einer Vergrößerung des Abstandes zwischen dem Substrat und der Rakelunterkante führt. Dies wird zu einer dickeren Klebschicht führen. Des Weiteren können dynamische Effekte, wie bspw. eine Schwingung der Rakel, auftreten, welche wiederum die Homogenität der Schicht negativ beeinflussen werden. Zusätzlich ist bei der Auswahl der Rakel darauf zu achten, dass diese gegenüber Reinigungsmitteln resistent ist, so dass überschüssiger Kleber hiervon entfernt werden kann. Klebstoff an Stellen auf dem Substrat, an denen dieser nicht benötigt wird, wird manuell entfernt.

\subsubsection{Lösungsprozess für die Fügung zweier Bauteile}

Der Lösungsprozess für die Fügung von zwei Bauteilen mit dem vorliegendem Mikromontageplatz ist in Abb. 27 schematisch dargestellt und wird sich im Wesentlichen aus den drei Hauptvorgängen Prozessvorbereitung, Klebschichtauftrag und Absetzen eines Bauteils auf die Klebschicht zusammensetzen.

Bevor die Bauteile dem Mikromontageplatz zugeführt werden, erfolgt deren Reinigung, um eine saubere und partikelfreie Oberfläche sicherzustellen. Hierfür können bspw. Reinigungsbäder eingesetzt werden. Im Anschluss wird das Zielbauteil mittig auf dem Vakuumteller fixiert. Sofern Haftvermittler für die Fügung eingesetzt werden sollen, können diese aufgetragen und durch die integrierte Heizmöglichkeit in der Montageplattform thermisch aktiviert werden (siehe Abb. 27 (a)).

Anschließend wird das Klebstoffauftragswerkzeug aus seiner seitlichen Ruhelage mit Hilfe des Haltearmes oberhalb des Substrates positioniert. Der Abstand von Rakelunterseite zu Substratoberfläche beträgt an dieser Stelle einige Millimeter. Durch das Lösen der zentralen Schraube gleitet die Rakel auf die Oberfläche des Substrates. Ein etwaiger Winkelversatz wird ausgeglichen und die Parallelität beider Oberflächen zueinander wird sichergestellt. Diese Position stellt die Nulllage dar. Die Schraube wird fest gezogen und mit Hilfe der Mikrometerstellschraube an dem Auftragswerkzeug wird die geforderte Klebschichtdicke als Abstandsmaß eingestellt. Mit Hilfe der X-Achse an der Montageplattform wird das Substrat unter der Rakel hindurch bewegt, so dass die Startposition des Rakelprozesses festgelegt wird. Es wird manuell Klebstoff in ausreichender Menge und Dicke über der geforderten Auftragsfläche verteilt. Im Anschluss erfolgt der Rakelvorgang. Die Montageplattform wird unterhalb der Rakel hindurch bewegt. Die Rakel streicht dabei den Klebstoff auf die geforderte Dicke ab. Sobald die Endposition des Rakelprozesses erreicht worden ist, wird das Auftragswerk- 
zeug seitlich weggeklappt und die Rakel gereinigt, sodass kein Klebstoff an ihr haften bleibt. Sofern kein Absetzen eines Bauteils gefordert ist, wenn bspw. Passivierungs- oder Maskierungsschichten auf Substraten zu erstellen sind, ist der Prozess an dieser Stelle beendet (siehe Abb. 27 (b)-(g)).

Ist jedoch ein Absetzen eines Bauteiles hierauf erforderlich, wird zunächst die Montageplattform abgesenkt, um ausreichend Platz für die Aufnahme eines Bauteils mit dem Vakuumsauggreifer zu schaffen. Nach der Aufnahme wird die Montageplattform in die Höhenposition gefahren, in der das Feinabsetzen mit dem Greifwerkzeug möglich ist. Die Beobachtungseinheit wird aus ihrer Ruhelage seitlich herangeklappt und die Ausrichtung zwischen dem Zielbauteil und dem abzusetzenden Bauteil erfolgt. Dabei beträgt der Abstand beider Bauteile wenige Millimeter. Es werden hierfür die drei Freiheitsgrade (X, Y und $\theta$ ) der Montageplattform genutzt. Sobald die Ausrichtung bzw. die Positionierung abgeschlossen ist, wird das abzusetzende Bauteil auf dem Zielbauteil / Substrat abgesetzt. Um die Aushärtung des Klebstoffes an dieser Stelle zu beschleunigen, kann die integrierte Heizmöglichkeit der Montageplattform genutzt werden. Bis zu der Aushärtung der Fügestelle verbleiben beide Bauteile in Kontakt, um ein etwaiges Verrutschen bei der Lösung des Sauggreifers zu verhindern. Nach der vollständigen Aushärtung wird die Vakuumversorgung beendet und sofern notwendig die Werkzeuge gereinigt (siehe Abb. 27 (h) \& (i)).

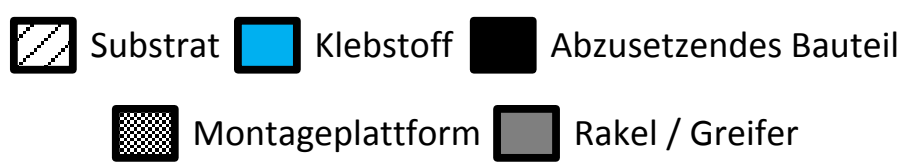

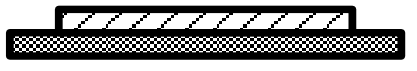

(a)

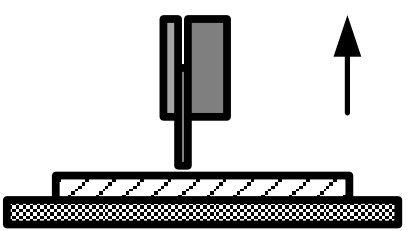

(d)

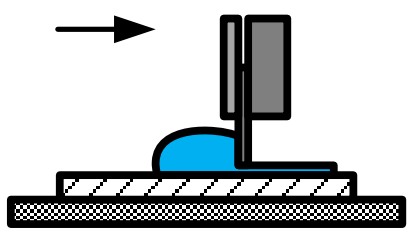

(g)

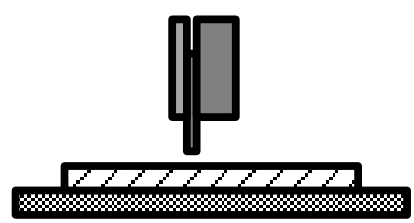

(b)

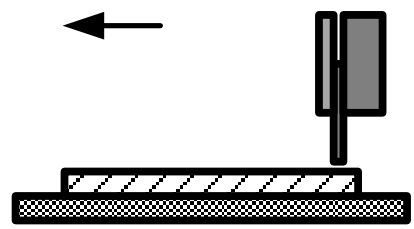

(e)

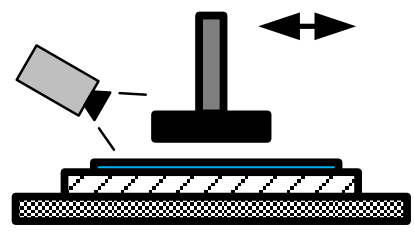

(h)

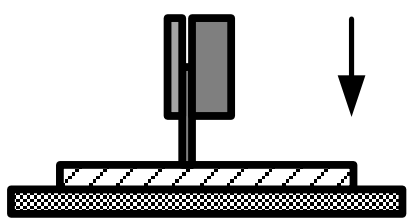

(c)

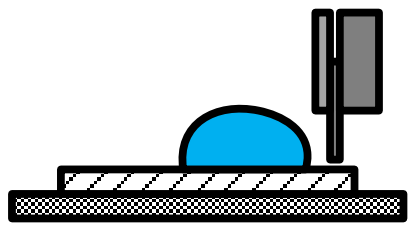

(f)

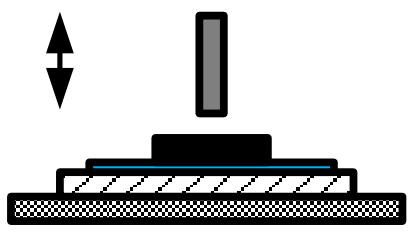

(i)

Abb. 27: Schematische Darstellung des Lösungsprozesses zur Fügung von zwei Bauteilen. (a) Ausgangszustand mit Substrat auf Montageplattform, (b) Positionierung der Rakeleinheit oberhalb des Substrates, (c) Rakel in Kontakt mit dem Substrat bringen, (d) gewünschte Klebschichtdicke einstellen, (e) Positionierplattform in Startposition bringen, (f) Klebstoff auftragen, (g) Klebstoff ganzflächig verteilen, (h) Ausrichtung beider Bauteile zueinander und (i) Absetzvorgang. 


\subsection{Zusammenfassende Betrachtung des eigenen Ansatzes}

In der Fertigung von Funktionsmodellen in geringen Stückzahlen für die Mikrosystemtechnik werden sowohl Mikrostrukturierungs- als auch Mikromontageoperationen benötigt. Dabei steht in vorliegendem Ansatz zusätzlich noch deren individuelle, kostengünstige und kurze Fertigung im Vordergrund.

Die Mikrostrukturierungsoperationen werden sowohl mit der Laserstrahlmaterialbearbeitung als auch mit einem Trennschleifprozess vorgenommen. Mit der Laserstrahlmaterialbearbeitung werden mit Ausnahme von senkrechten Kanälen alle Strukturen in dem Siliziumsubstrat gefertigt. Sofern senkrechte Kanäle mit geringer Rauheit an den Seitenwänden gefordert sind, wird der Trennschleifprozess hinzugezogen. Die Vorteile der Laserstrahlmaterialbearbeitung sind, dass es nahezu keine Einschränkung in der Formenvielfalt gibt und dass unterschiedliche Designvariationen in CAD Programmen erstellt und direkt in die Lasersystemsteuerung importiert werden können. Es wird der Einfluss von Bearbeitungsparametern auf das Abtragsergebnis aufgezeigt, welche nicht von der eigentlichen Laserstrahlquelle abhängen. Dies sind die Fokusposition, der Vorschub und der Linienabstand. Das Ergebnis aus den Untersuchungen ist, dass die aufgezeigten Zusammenhänge auf jedes Lasersystem übertragen werden können. Die Parameter werden hinsichtlich einer hohen Materialabtragsrate, einer hohen Formtreue und einer geringen Rauheit optimiert. Um der Forderung der Erstellung von Kanälen mit senkrechten Seitenwänden und gleichzeitig auch mit geringer Rauheit nachzukommen, wird das Trennschleifen mit einer Wafersäge vorgenommen. Im Gegensatz zu dem standardisierten Heraustrennen von Dies aus Substraten, werden Nuten mit definierter Tiefe und Breite erstellt. Hierfür wird ein zweistufiger Prozess genutzt. Dabei werden mitunter auch überlappende Sägeschnitte eingesetzt, mit denen es möglich ist, die Breite der Kanäle unabhängig von der Sägeblattbreite zu realisieren. Die Bestimmung der Position der Substratoberfläche erlaubt das anschließende Verfahren der Sägespindel in die gewünschte Tiefe der Kanäle. Es können mit dem Trennschleifen nur gerade Schnitte ausgeführt werden; jedoch ist dieser Sachverhalt für die Kanalherstellung bei Mikrotropfenerzeugern nicht von Bedeutung und der Vorteil einer senkrechten Seitenwand mit geringer Rauheit dominiert.

Für die notwendigen Mikromontageoperationen wird ein an die Anforderungen angepasster Mikromontageplatz entwickelt, welcher das Hauptziel verfolgt, planare Substrate über eine dünne, homogene und ganzflächige Klebstoffschicht zu fügen. Der Mikromontageplatz wird direkt in den Fertigungsprozess von Mikrosystemen eingebunden und wird sich durch einen vertikalen, kompakten und vor allem modularen Aufbau auszeichnen. An seinen Seitenwänden befinden sich Gewindebohrungen und Führungsschienen, an denen weitere Werkzeuge über standardisierte Haltearme angebracht werden können. Das abzusetzende Bauteil befindet sich auf einem Greif- / Absetzwerkzeug, welches durch ein Schubkurbelgetriebe bei kleinen Kurbelwinkeln ein Absetzen mit Feingefühl ermöglicht. Die Ausrichtung des Zielbauteils erfolgt mit einer Montageplattform in X-, Y- und $\theta$-Richtung. Die Fixierung beider Bauteile erfolgt mit Vakuum. Wird zusätzlich eine dünne, homogene und ganzflächige Klebstoffschicht gefordert, so wird das Klebstoffauftragswerkzeug mit Hilfe des Haltearms hinzugezogen. Das Arbeitsprinzip entspricht dabei dem Abziehen von Klebstoff auf dem Zielbauteil mit einer Rakelklinge. Mit einer Mikrometerstellschraube an einer Linearachse wird hierbei die Klebschichtdicke eingestellt. Die Aushärtung des Klebstoffes kann mit einer integrierten Heizmöglichkeit in der Montageplattform beschleunigt werden. Der eigene Ansatz des Mikromontageplatzes zeigt, dass in einem Werkzeug alle benötigten Funktionen für die Fügung von zwei Bauteilen an einem Ort zur Verfügung gestellt werden. 


\section{Unterscheidungsmerkmale zum Stand der Technik}

In dem vorgestellten Ansatz für die Fertigung von Funktionsmodellen werden sowohl die Werkzeuge für die notwendige Mikrostrukturierung als auch für die Mikromontage beschrieben. Es ergeben sich gegenüber dem Stand der Technik folgende Unterscheidungsmerkmale:

Kurze Herstellungszeit von geringen Stückzahlen: Es werden Funktionsmodelle in geringen Stückzahlen gefertigt, ohne dabei den mikroelektronischen Produktionszyklus zu durchlaufen. Die mikroelektronische Fertigung kann je nach Komplexität der Strukturen eine lange Prozesszeit mit sich bringen (Fujita, 2007; Benilov et al., 2008; Pimpin und Srituravanich, 2011), welche sich nur bei großen Stückzahlen durch die Batchprozessierung relativiert. Vorliegend findet ein direkter Ablationsprozess sowohl mit einem Lasersystem als auch mit einer Wafersäge statt. Es wird für die Mikrostrukturierung nicht auf die Prozessfolge aus Schichtabscheidung, -strukturierung und -ätzung sowie der notwendigen Beschaffung von Maskensätzen zurückgegriffen. Der Mikromontageplatz besitzt eine geringe Rüstzeit. Das Ergebnis ist, dass geringe Stückzahlen eines Mikrosystems in kurzer Zeit hergestellt werden können.

Niedrige Fertigungskosten bei geringen Stückzahlen: Die mikroelektronische Fertigungstechnik zeichnet sich durch die Batchprozessierbarkeit aus. Es ergeben sich bei hohen Stückzahlen eines identischen Mikrosystems niedrige Fertigungskosten trotz hoher Unterhalts- und Anschaffungskosten der Fertigungsumgebung. Lawes (2007) und Schwesinger (2015) nennen hierzu Anschaffungskosten von bspw. Trockenätzanlagen in Höhe von mindestens $500 \mathrm{~T} €$, Mask Alignern von mindestens $200 \mathrm{~T} €$ sowie von Spin Coatern von mindestens $50 \mathrm{~T} €$. Hinzu kommen noch Anschaffungskosten für den Reinraum in Höhe von ca. $3 \mathrm{~T} € / \mathrm{m}^{2}$ (Tadigadapa und Najafi, 2003). Bei kleinen Stückzahlen sind diese Verfahren unrentabel, da die Kosten nicht durch die hohen Stückzahlen kompensiert werden (Abgrall und Gué, 2007; Fujita, 2007; Ashida et al., 2010). In dieser Arbeit werden für die Mikrostrukturierung ein Lasersystem und eine Wafersäge benötigt. Es sind weder ein Reinraum noch weitere Gerätschaften (Ätzanlagen, Mask Aligner, Spin Coater, Hotplates etc.) notwendig. Das Mikromontagesystem zeichnet sich durch einen kostengünstigen Aufbau aus. Es werden keine motorbetriebenen Achsen eingesetzt und als Beobachtungseinheiten werden Videomikroskope hinzugezogen.

Individuelle und angepasste Fertigung: Mit den eingesetzten Werkzeugen werden Substrate individuell strukturiert. Durch den Einsatz der Lasermikrobearbeitung ist es möglich, beliebige Freiformen in Substraten zu erstellen. Im Gegensatz zu konventionellen Klebstoffauftragsverfahren in Mikromontageanlagen erfolgt ein ganzflächiger Auftrag. Das entwickelte Fertigungsverfahren kennzeichnet sich durch eine an die Randbedingungen von Funktionsmodellen angepasste Fertigungsgenauigkeit im Mikrometerbereich aus.

Geringer Schulungsaufwand: Die Mikrostrukturierung von Substraten wird sowohl mit unterschiedlichen Geräten (Ätzanlagen, Mask Aligner, Spin Coater, Hotplates etc.) als auch mit vielen Chemikalien / Ätzmedien vorgenommen. Vorliegend erfolgt die Mikrostrukturierung lediglich mit einem Lasersystem und einer Wafersäge. Ergänzend werden Reinigungslösungen bei Bedarf hinzugezogen. Hieraus ergibt sich ein geringerer Schulungsaufwand der Operatoren, da der Umgang mit Gefahrstoffen, wie bspw. Ätzmedien, vermieden wird und lediglich zwei Geräte zu bedienen sind. Darüber hinaus zeichnet sich das Mikromontagesystem aufgrund seiner reinen mechanischen Bedienung als intuitiv bedienbar aus. 


\section{Realisierung}

\subsection{Werkzeuge für die Strukturierung von Substraten}

\subsubsection{Laserstrahlmaterialbearbeitung mit Nanosekunden Laserstrahlquellen}

Die Untersuchungen zu dem Laserstrahlmaterialabtrag werden an zwei Lasersystemen durchgeführt. Beide Systeme bestehen aus einem Nd:YAG Festkörperlaser, einem Galvanometerscanner mit Planfeldoptik (F- $\Theta-O p t i k)$ und aus motorbetriebenen Achsen sowohl für die horizontale als auch für die vertikale Ausrichtung des Bauteils. Die Untersuchungen zu dem Laserstrahlschneiden werden an dem frequenzverdreifachten UV Lasersystem Gator $(\lambda=355 \mathrm{~nm})$ der Firma Lambda Physik und die Untersuchungen zu dem ebenenweisen Laserstrahlmaterialabtrag an dem IR Lasersystem LS $2000 / 8000(\lambda=1064 \mathrm{~nm})$ der Firma Laser Systems vorgenommen (siehe Abb. 28). Der Grund für die Wahl zweier Lasersysteme ergibt sich daraus, dass während dieser Arbeit an dem Lasersystem Gator eine länger anhaltende Reparatur durchgeführt worden ist, so dass die Untersuchungen für den ebenenweisen Laserstrahlmaterialabtrag an dem IR Lasersystem fortgeführt worden sind. Abb. 29 zeigt bildhaft die in (7.1) geschilderte formelmäßige Sachlage auf, dass geringere Wellenlängen zu einer höheren Strukturauflösung führen, da sich hierdurch der Fokusdurchmesser reduziert.

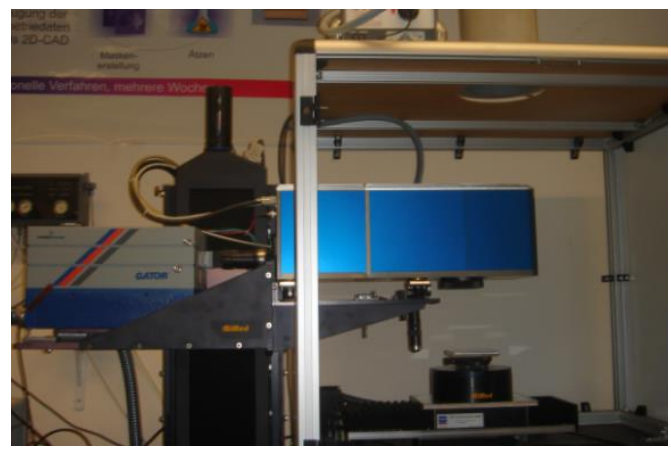

(a)

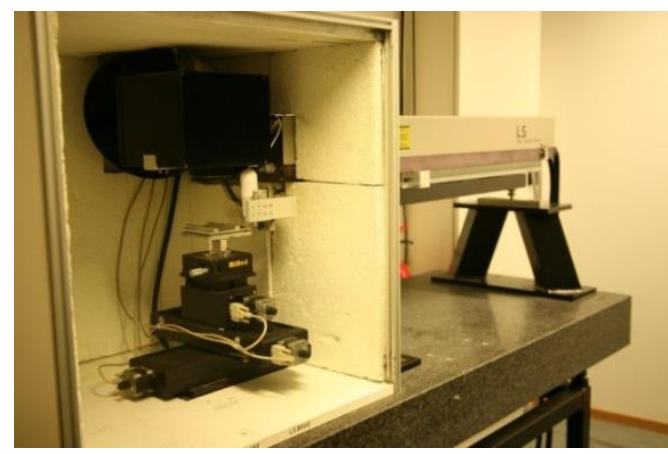

(b)

Abb. 28: Verwendete Lasersysteme für die Untersuchungen zu dem Laserstrahlmaterialabtrag. (a) UV Lasersystem Gator von Lambda Physik und (b) IR Lasersystem LS 2000 / 8000 von Laser Systems.

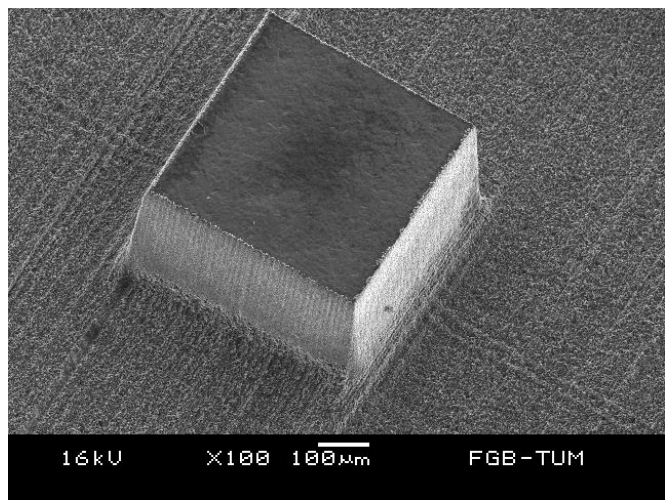

(a)

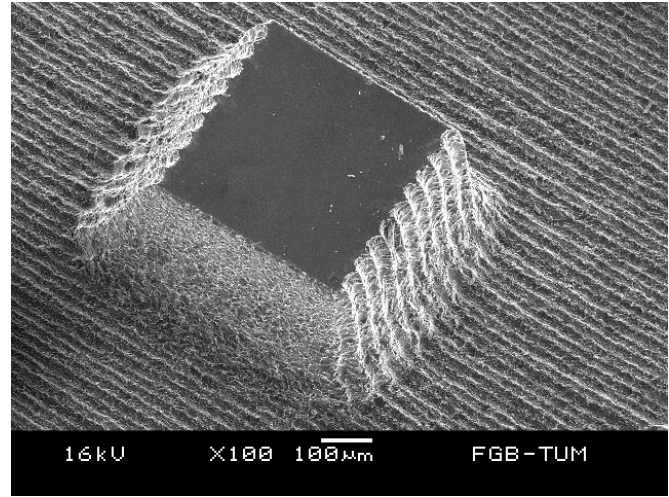

(b)

Abb. 29: Rasterelektronenmikroskopische Aufnahmen für den Einfluss der Wellenlänge auf die Strukturauflösung. Identische Inseln sind in Silizium bei gleicher Bearbeitung abladiert, allerdings in (a) mit einem UV und in (b) mit einem IR Lasersystem. Entnommen aus Wolf (2003). 
Beide Systeme besitzen einen Güteschalter (Q-Switch), durch den sehr hohe Pulsspitzenleistungen bei vergleichsweise geringen mittleren Leistungen erreicht werden. Der Betrieb der Laser erfolgt gepulst im Nanosekundenbereich. Die Systeme werden über herstellereigene Programmiersprachen gesteuert, welche es erlauben, einfache Geometrien zu erzeugen und auch Laserparameter zu verändern. Komplexe Freiformen werden hingegen in CAD Programmen erstellt und als HPGL Datei (Hewlett-Packard Graphics Language) in die Steuerungssoftware des Lasersystems / Galvanometerscanners importiert. Die Bahnsteuerung des Laserspots erfolgt mit den Galvanometerscannern, welche jeweils ein Bearbeitungsfeld von 60 x $60 \mathrm{~mm}^{2}$ mit den verwendeten F- $\Theta-O p t i k e n$ abdecken. Die maximale Scangeschwindigkeit bei dem UV System beträgt $300 \mathrm{~mm} / \mathrm{s}$ und bei dem IR System $10000 \mathrm{~mm} / \mathrm{s}$. Tabelle 1 gibt die wichtigsten nominalen technischen Daten der beiden Systeme wieder.

In jedem Fertigungssystem ist ein Kreuztisch für die horizontale Ausrichtung des Werkstückes vorhanden. Das UV System Gator verfügt hierbei über den Kreuztisch LTB 170 der

Tabelle 1: Nominale technische Daten der verwendeten Laserbearbeitungssysteme.

\begin{tabular}{|c|c|c|}
\hline & UV System Gator & IR System LS 2000 / 8000 \\
\hline \multicolumn{3}{|l|}{ Laserstrahlquelle } \\
\hline $\begin{array}{l}\text { Modell, } \\
\text { Hersteller }\end{array}$ & $\begin{array}{l}\text { Gator 355-2, } \\
\text { Lambda Physik }\end{array}$ & $\begin{array}{l}\text { LS } 2000 / 8000, \\
\text { Laser Systems }\end{array}$ \\
\hline Lasermedium & Nd:YAG, gütegeschaltet & Nd:YAG, gütegeschaltet \\
\hline Anregung & Laserdiode & Krypton Bogenlampe \\
\hline Wellenlänge [nm] & 355 & 1064 \\
\hline Pulsfrequenz [kHz] & $8-12$ & $0,1-50$ \\
\hline Pulsdauer [ns] & $15 \pm 3$ & $100-150$ (Wolf, 2003) \\
\hline Maximale Leistung [W] & 3 & 30 (Scheuenpflug, 2011) \\
\hline Beugungsmaßzahl $\mathrm{M}^{2}$ & $\approx 1,2$ & $>2$ (Wolf, 2003) \\
\hline \multicolumn{3}{|l|}{ Scankopf } \\
\hline $\begin{array}{l}\text { Modell, } \\
\text { Hersteller }\end{array}$ & $\begin{array}{l}\text { Elephant, } \\
\text { Arges }\end{array}$ & $\begin{array}{l}\text { SK1010, } \\
\text { Scanlab }\end{array}$ \\
\hline Bearbeitungsfeld $\left[\mathrm{mm}^{2}\right]$ & $60 \times 60$ & $60 \times 60$ \\
\hline Brennweite (F- $\theta$-Optik) $[\mathrm{mm}]$ & 100 & 100 \\
\hline Maximale Scangeschwindigkeit $[\mathrm{mm} / \mathrm{s}]$ & 300 & 10000 \\
\hline Bearbeitungsdurchmesser Silizium [ $\mu \mathrm{m}]$ & 15 (Liess, 2006) & > 50 (Wolf, 2003) \\
\hline \multicolumn{3}{|l|}{ Z-Achse } \\
\hline $\begin{array}{l}\text { Modell, } \\
\text { Hersteller }\end{array}$ & $\begin{array}{l}\text { PMT 200-320, } \\
\text { Steinmeyer Mechatronik }\end{array}$ & $\begin{array}{l}\text { HVM } 100, \\
\text { OWIS }\end{array}$ \\
\hline Maximaler Verfahrweg [mm] & 320 & 30 \\
\hline $\begin{array}{l}\text { Absolute Genauigkeit } \\
{[\mu \mathrm{m} / 100 \mathrm{~mm} \text { Verfahrweg] }}\end{array}$ & 3 & 20 \\
\hline
\end{tabular}


Firma SKF mit einem Verfahrbereich von 310 x $220 \mathrm{~mm}^{2}$ bei einer Genauigkeitsklasse von $\pm 5 \mu \mathrm{m}$. Im IR System LS $2000 / 8000$ ist der Kreuztisch LIMES 120 der Firma OWIS verbaut, der einen Verfahrbereich von 150 × $150 \mathrm{~mm}^{2}$ bei einer absoluten Genauigkeit von $16 \mu \mathrm{m}$ auf $100 \mathrm{~mm}$ Verfahrstrecke erlaubt. Zusätzlich ist in der UV Laserbearbeitungsanlage ein angetriebener Drehtisch integriert.

Die Einstellung des Fokus erfolgt durch einen gezielten Fokusfindungsvorgang. Beide Systeme enthalten neben den Kreuztischen auch jeweils eine motorbetriebene Höhenachse, mit welcher der Abstand zwischen Werkstück und F- $\Theta-O p t i k$ des Galvanometerscanners eingestellt wird. Zusätzlich beinhaltet der Scanner des UV Systems auch eine integrierte optische Höhenachse. Durch das Setzen einzelner Laserpunkte mit verschiedenen Werkstückabständen wird der Fokus auf der Werkstückoberfläche bestimmt. Abb. 30 zeigt hierzu ein Muster für den Fokusfindungsvorgang. Die Höhenachse wird hierbei zwischen jedem Laserpunkt um $50 \mu \mathrm{m}$ verfahren. Befindet sich der Fokus unter- oder auch oberhalb des Werkstückes, ergeben sich große Punktdurchmesser und es findet kaum Materialabtrag auf der Oberfläche statt. Im Ergebnis bedeutet dies, dass die korrekte Fokusposition als diejenige definiert wird, an der der Punktdurchmesser auf der Werkstückoberfläche am geringsten ist. Für eine noch genauere Fokuslagenbestimmung kann bei Bedarf das Intervall der Abstände verringert werden.

\subsubsection{Fertigung senkrechter Kanäle mit einer Wafersäge}

Für die Herstellung von senkrechten Kanälen in Substraten wird das Trennschleifverfahren verwendet. Der Vorteil des Trennschleifens mit einer Wafersäge bei der Erstellung von Kanälen in Substraten ist, dass Kanäle mit senkrechten Seitenwänden und gleichzeitig mit geringer Rauheit erstellt werden. Vor allem in mikrofluidischen Anwendungen, wie bspw. bei Düsen in einem Mikrotropfenerzeuger, ist ein definierter Kanalquerschnitt mit geringer Rauheit essentiell für die reproduzierbare Tropfenbildung bei geringer Reibung. Abb. 31 zeigt hierzu die Kanalerstellung sowohl mittels Laserstrahlung als auch mittels Trennschleifens mit einer Wafersäge auf. Deutlich ist der Unterschied bzgl. der Rauheit an den Seitenflanken und der Form der Seitenwände erkennbar.

Wie bereits in Kapitel 7 beschrieben, werden für das Trennschleifen mit einer Wafersäge für Silizium Sägeblätter mit einer metallischen Nickelbindung und geringem Schleifkorn benötigt. Hierdurch werden eine vergleichsweise hohe Blattstandzeit und feine Schnitte mit gerin-

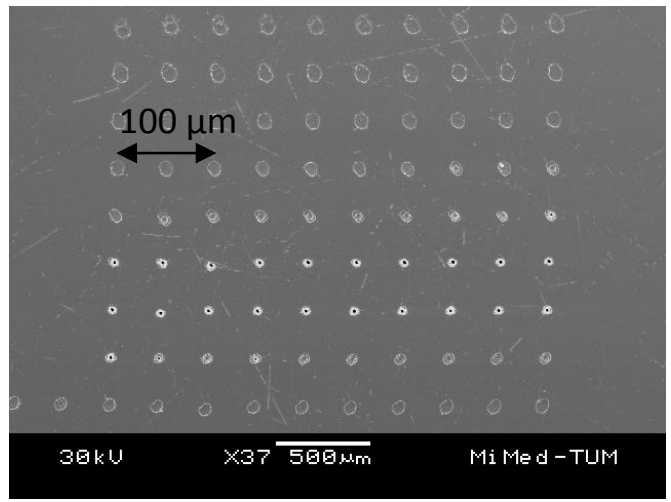

(a)

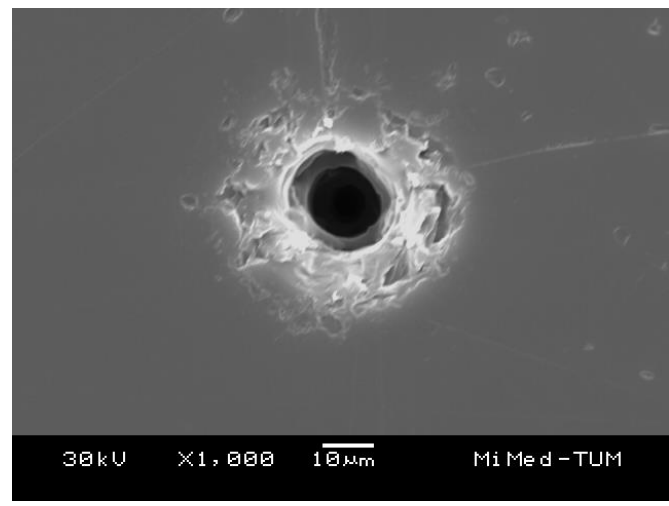

(b)

Abb. 30: Rasterelektronenmikroskopische Aufnahmen für die Bestimmung des Fokus. (a) Laserpunktemuster im Abstand von $50 \mu \mathrm{m}$ über einem Arbeitsbereich von 4,45 mm und (b) Laserpunkt im Fokus (entnommen aus Albrecht (2012)). Die Laserpunkte sind mit dem IR Laser LS 2000 / 8000 bei einer mittleren Leistung von $2 \mathrm{~W}$ erstellt worden. 
gem Chipping erzielt. Aus diesem Grund wird das Sägeblatt FTB R46 45 x 100 von der Firma Mitsubishi Corporation gewählt. Dieses besitzt eine Körnung zwischen 4 und $6 \mu \mathrm{m}$, eine Blattdicke von $45 \mu \mathrm{m}$ und einen minimalen Blattüberstand von 1,1 mm.

Neben der korrekten Wahl des Sägeblattes gilt es auch, den Einfluss der Schnittparameter (Drehzahl und Vorschub) auf das Schnittergebnis zu beachten. Kagerer et al. (2012a) beschreiben hierzu den Einfluss von Drehzahl und Vorschub auf das Chipping und auf die Welligkeit des Schnittes. Hierbei wird gezeigt, dass die Welligkeit eines Schnittes mit zunehmendem Vorschub steigt. Der Grund hierfür ist, dass das Sägeblatt durch seinen Blattüberstand dem Werkstück seitlich der Schnittkerbe ausweicht, da auf die Schneide hohe Scherkräfte wirken. So wird die Welligkeit von $30 \mu \mathrm{m}$ bei einem Vorschub von $50 \mathrm{~mm} / \mathrm{s}$ auf unter $2 \mu \mathrm{m}$ bei Vorschüben $<1,5 \mathrm{~mm} / \mathrm{s}$ reduziert. Eine Verringerung des Vorschubes führt ebenfalls zu einem geringeren Chipping. Ein hoher Vorschub leitet in das Substrat hohe Kräfte ein, so dass sich Aussprünge weiter ausbreiten. Dabei wird das Chipping von $30 \mu \mathrm{m}$ bei einem Vorschub von $50 \mathrm{~mm} / \mathrm{s}$ auf unter $6 \mu \mathrm{m}$ bei Vorschüben $<1,5 \mathrm{~mm} / \mathrm{s}$ minimiert (siehe hierzu Abb. 32). Des Weiteren wird aufgezeigt, dass mit einer Erhöhung der Spindeldrehzahl das Chipping ebenfalls verringert wird. Wird die Drehzahl bei gleichem Vorschub erhöht, bleibt die gesamte Abtragsrate konstant, jedoch verringert sich der jeweilige Materialabtrag pro Umlauf des Blattes. Dies bedeutet, dass die zum Abtragen benötigte Energie sich auf mehrere Umläufe verteilt, so dass im Ergebnis die auf das Substrat einwirkende Belastung reduziert wird. Hierdurch wird das auftretende Chipping mit steigender Drehzahl reduziert. An dieser Stelle gilt

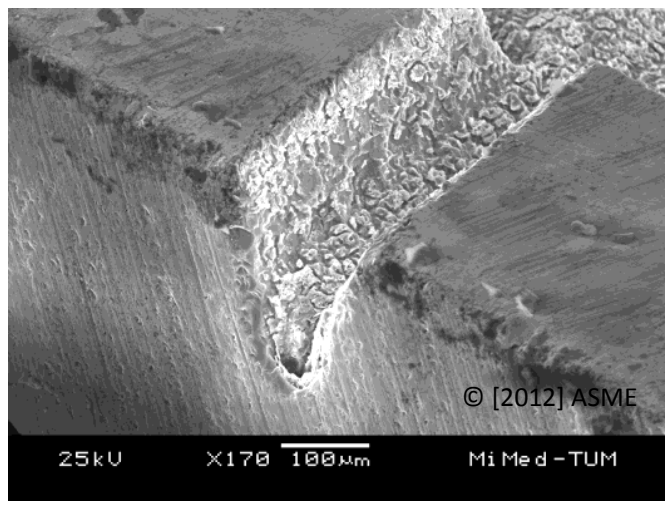

(a)

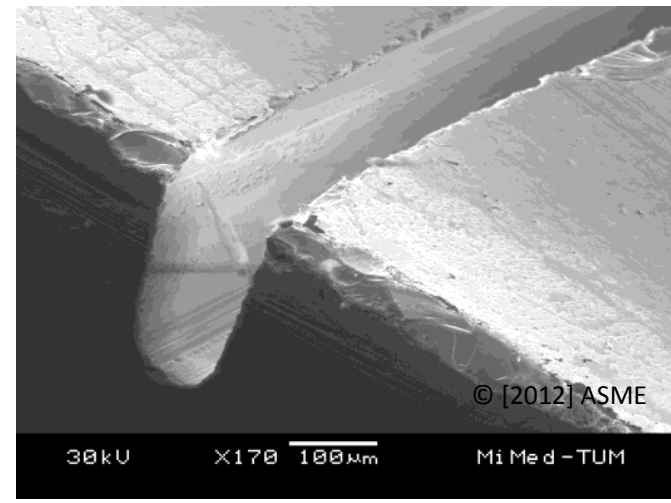

(b)

Abb. 31: Rasterelektronenmikroskopische Aufnahmen von erstellten Kanälen in Silizium in (a) mit dem IR Lasersystem LS 2000 / 8000 und in (b) mit einer Wafersäge. Entnommen aus Kagerer et al. (2012a).

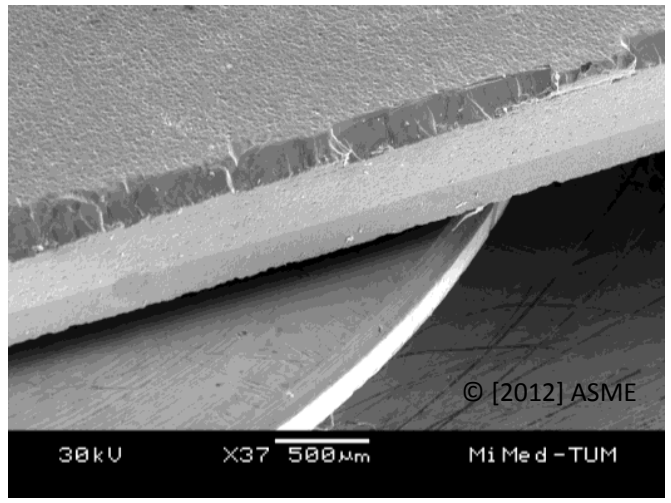

(a)

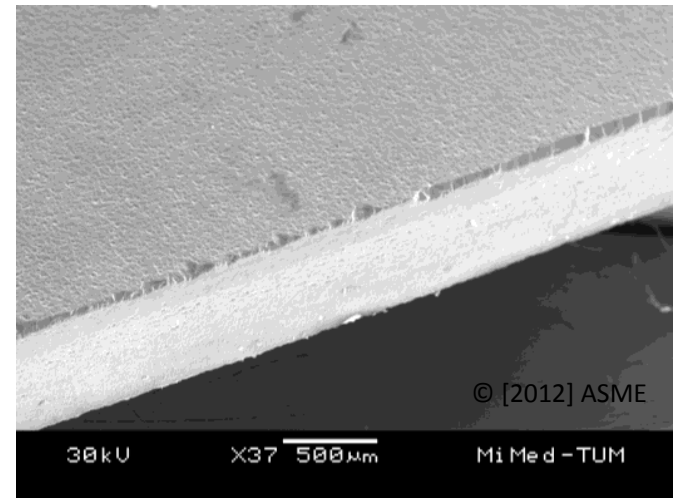

(b)

Abb. 32: Rasterelektronenmikroskopische Aufnahmen von auftretendem Chipping in Silizium in (a) mit einem Vorschub von $50 \mathrm{~mm} / \mathrm{s}$ und in (b) mit $0,75 \mathrm{~mm} / \mathrm{s}$. Entnommen aus Kagerer et al. (2012a). 
es zu erwähnen, dass diese Untersuchungen mit einem Universalsägeblatt mit einer Körnung von $15 \mu \mathrm{m}$ und einer Blattdicke von $100 \mu \mathrm{m}$ durchgeführt worden sind. Bei Verwendung eines reinen Siliziumsägeblattes werden sowohl das auftretende Chipping als auch die Welligkeit des Schnittes nochmals reduziert.

Für das Trennschleifen steht die Wafersäge DAD321 von der Firma DISCO HI-TEC EUROPE zur Verfügung (siehe Abb. 33 (a) \& (b)). Die Säge ist selbstauswuchtend und besitzt drei translatorische (X, Y und Z) Achsen und eine rotatorische $(\theta)$ Achse. Werkstücke bis zu einem Durchmesser von 6 Zoll werden bearbeitet. Das zu bearbeitende Werkstück wird für das Trennschleifen auf einer Sägefolie mit Klebschicht befestigt. Werden sehr hohe Klebkräfte, vor allem bei dünnen und spröden Substraten, benötigt, bieten UV empfindliche Sägefolien gegenüber normalen Blue Tapes deutliche Vorteile. Ihre hohe Haftkraft im unbelichteten $\mathrm{Zu}$ stand erlaubt eine sichere Fixierung auch von kleinsten Dies. Hierbei werden Ausbrüche, die beim Trennschleifen durch eine mögliche Bewegung der Werkstücke entstehen, reduziert. Auf der anderen Seite besteht die Möglichkeit, die Klebkraft mit einer UV Belichtung deutlich zu reduzieren, so dass die Dies nach dem Trennschleifprozess bruchfrei abgezogen werden. Die Kombination aus hoher Klebkraft und bruchfreier Demontage der Dies begründet den Einsatz einer UV empfindlichen Sägefolie. In vorliegendem Projekt wird die Sägefolie 1027R der Firma Ultron Systems verwendet. Diese weist eine Gesamtdicke von $175 \pm 20 \mu \mathrm{m}$ auf (siehe Abb. 33 (c)). Die Adhäsion von Silizium beträgt dabei vor der UV Belichtung $2080 \mathrm{~g} / 25 \mathrm{~mm}$ und nach der UV Belichtung lediglich $28 \mathrm{~g} / 25 \mathrm{~mm}$. Somit wird ein bruchund beschädigungsfreies Abziehen der Bauteile von der Sägefolie gewährleistet.

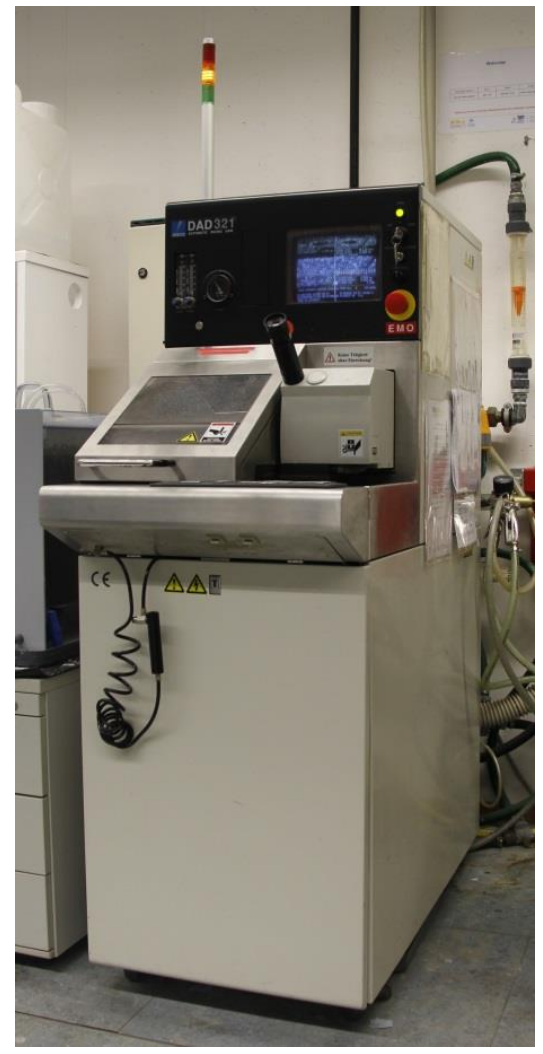

(a)

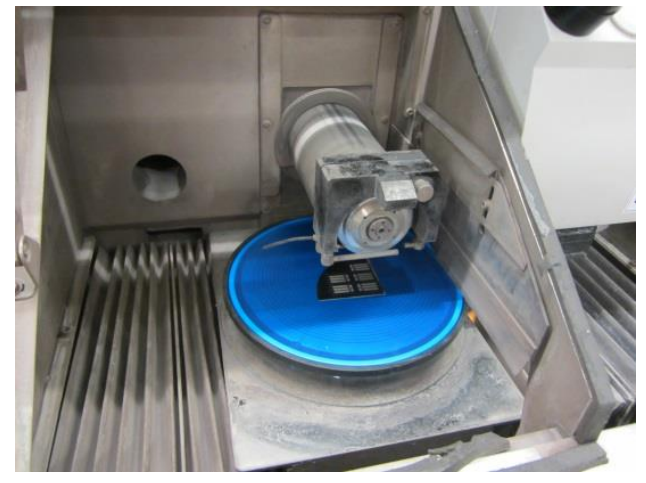

(b)

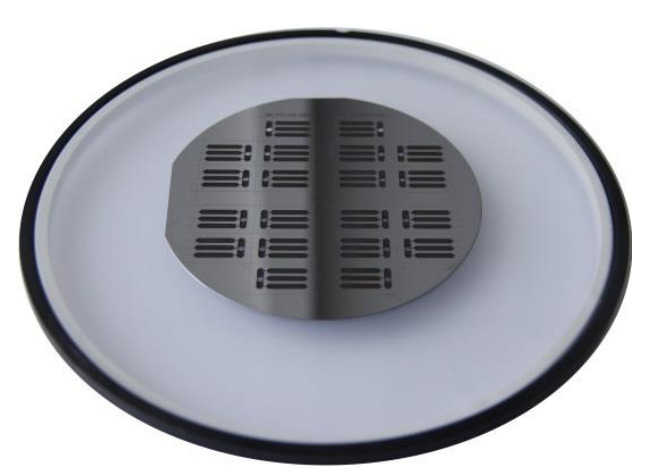

(c)

Abb. 33: Werkzeug für die Erstellung senkrechter Kanäle in Substraten. (a) Wafersäge DAD321 von DISCO HI-TEC EUROPE, (b) Bearbeitungsraum der Wafersäge mit Substrat auf standardisiertem Blue Tape und (c) aufgeklebter 4 Zoll Siliziumwafer auf UV Licht löslicher Sägefolie um den Spannring. 
Die Programmierung der Schnittprogramme erfolgt in einer proprietären CAM Sprache (computer-aided manufacturing). Die Wafersäge stellt zwei Bearbeitungsmodi zur Verfügung: Den DAC und den DAD Modus. Der DAD Modus stellt den Modus für das standardisierte Heraustrennen von Dies dar. Hierbei werden neben den Prozessparametern (Drehzahl, Vorschub und Absenkung der Spindel), auch die Werkstückgröße / -form und die Sägestraßen in X- und Y-Richtung definiert. Ausgangspunkt der Schnitte für die Wafersäge stellt in diesem Modus ein zentriertes Werkstück, bezogen auf die Länge der Schnitte, auf dem Vakuumteller dar. Dieser Modus eignet sich daher nicht für die vorgegebene Anwendung, um an einer beliebigen Stelle auf dem Werkstück unterschiedlich tiefe, breite und lange Kanäle zu erstellen. Daher wird für die Erstellung von Kanälen mit definierter Tiefe und Breite der DAC Modus der Wafersäge verwendet. Mit diesem Modus sind Schnitte möglich, die an einer definierten Position abgesetzt und nach einer vorgegebenen Wegstrecke erneut ausgeführt werden. Die Programmierung der Schnitte im DAC Modus ist gegenüber dem DAD Modus komplexer, da jeder einzelne Schnitt mit den entsprechenden Parametern zu versehen ist. Lediglich die Drehzahl der Spindel wird zentral für alle Schnitte vorgegeben. Allerdings ergibt sich aus dieser Komplexität auch die Freiheit, die Breiten, Tiefen und Längen von Kanälen individuell anzupassen. So besteht die Möglichkeit, den Versatz zwischen jedem Schnitt, den Vorschub, die Länge, die Tiefe und auch die Absenkgeschwindigkeit der Spindel in das Substrat für jeden auszuführenden Schnitt einzustellen. Durch diese hohe Gestaltungsfreiheit werden Schnitte mit definierter Tiefe, Breite und Länge an jeder gewünschten Stelle auf dem Werkstück ermöglicht. Nach der Ausrichtung des Werkstückes erfolgt das Setzen der Startposition des Schnittes. Hierfür steht jeweils ein Fadenkreuz sowohl für ein Videomikroskop als auch für ein optisches Mikroskop zur Verfügung. Der Materialabtrieb aus dem Schnittgraben wird durch eine ausgerichtete Prozesswasserspülung unterstützt.

Tabelle 2 fasst die technischen Daten der eingesetzten Wafersäge zusammen. Wie aus Kapitel 7 hervorgeht, ist für das Schnittergebnis neben dem Sägeblatt vor allem auch der Vorschub und die Drehzahl der Spindel verantwortlich. Für beide Parameter besteht die Möglichkeit, diese mit vorliegendem Werkzeug über eine große Bandbreite zu variieren.

Tabelle 2: Nominale technische Daten der verwendeten Wafersäge DAD321.

\begin{tabular}{|l|l|}
\hline Spezifikation & Wert \\
\hline $\begin{array}{l}\text { Modell, } \\
\text { Hersteller }\end{array}$ & $\begin{array}{l}\text { DAD321, } \\
\text { DISCO HI-TEC EUROPE }\end{array}$ \\
\hline Maximaler Werkstückdurchmesser [Zoll] & 6 \\
\hline Maximale Schnittweite X-Achse $[\mathrm{mm}]$ & 192 \\
\hline Vorschubgeschwindigkeit X-Achse $[\mathrm{mm} / \mathrm{s}]$ & $0-300$ \\
\hline Maximale Schnittweite Y-Achse $[\mathrm{mm}]$ & 162 \\
\hline Absolute Genauigkeit Y-Achse $[\mu \mathrm{m} / 160 \mathrm{~mm}$ Verfahrweg] & 5 \\
\hline Maximale Verfahrgeschwindigkeit Y-Achse $[\mathrm{mm} / \mathrm{s}]$ & 60 \\
\hline Hub Z-Achse [mm] & 32 \\
\hline Wiederholgenauigkeit Z-Achse $[\mu \mathrm{m}]$ & 1 \\
\hline Rotationsgeschwindigkeit der Spindel $[\mathrm{U} / \mathrm{min}]$ & $3000-40000$ \\
\hline
\end{tabular}




\subsection{Mikromontageplatz zur Fügung zweier Bauteile}

Der Mikromontageplatz besteht im Wesentlichen aus sieben Komponenten: Dies sind der Rahmen mit den Seitenwänden, die Montageplattform, das Greif- / Absetzwerkzeug, die Höhenachse für die Montageplattform, die Beobachtungseinheit, das Auftragswerkzeug für Klebstoff und die weitere Peripherie. Der Mikromontageplatz nimmt dabei eine Grundfläche von $24 \times 24 \mathrm{~cm}^{2}$ bei einer Gesamthöhe von $50 \mathrm{~cm}$ ein. Nachfolgend werden seine einzelnen Funktionsgruppen beschrieben.

\subsubsection{Gestell des Mikromontageplatzes}

Wie bereits in Kapitel 7 beschrieben, ist das Gestell eines der zentralen Bauteile des Mikromontageplatzes, da es alle weiteren Baugruppen miteinander verbindet. Abb. 34 zeigt dieses auf. Es besteht aus einer Bodenplatte aus rostfreiem Stahl, zwei Seitenwänden und einer Rückwand, die jeweils aus Aluminium gefertigt werden. Die daran angebrachte Höhenachse dient der vertikalen Positionierung der Montageplattform.

Die Bodenplatte ist massiv ausgeführt, so dass durch den im Ergebnis niedrig liegenden Schwerpunkt des Mikromontageplatzes dieser hierdurch mehr Standfestigkeit erlangt. Zusätzlich befinden sich an den Ecken der Bodenplatte Durchgangslöcher, so dass der Mikromontageplatz an einem Arbeitstisch befestigt werden kann und ein versehentliches Kippen mit der Gefahr einer Beschädigung von Werkstücken ausgeschlossen wird.

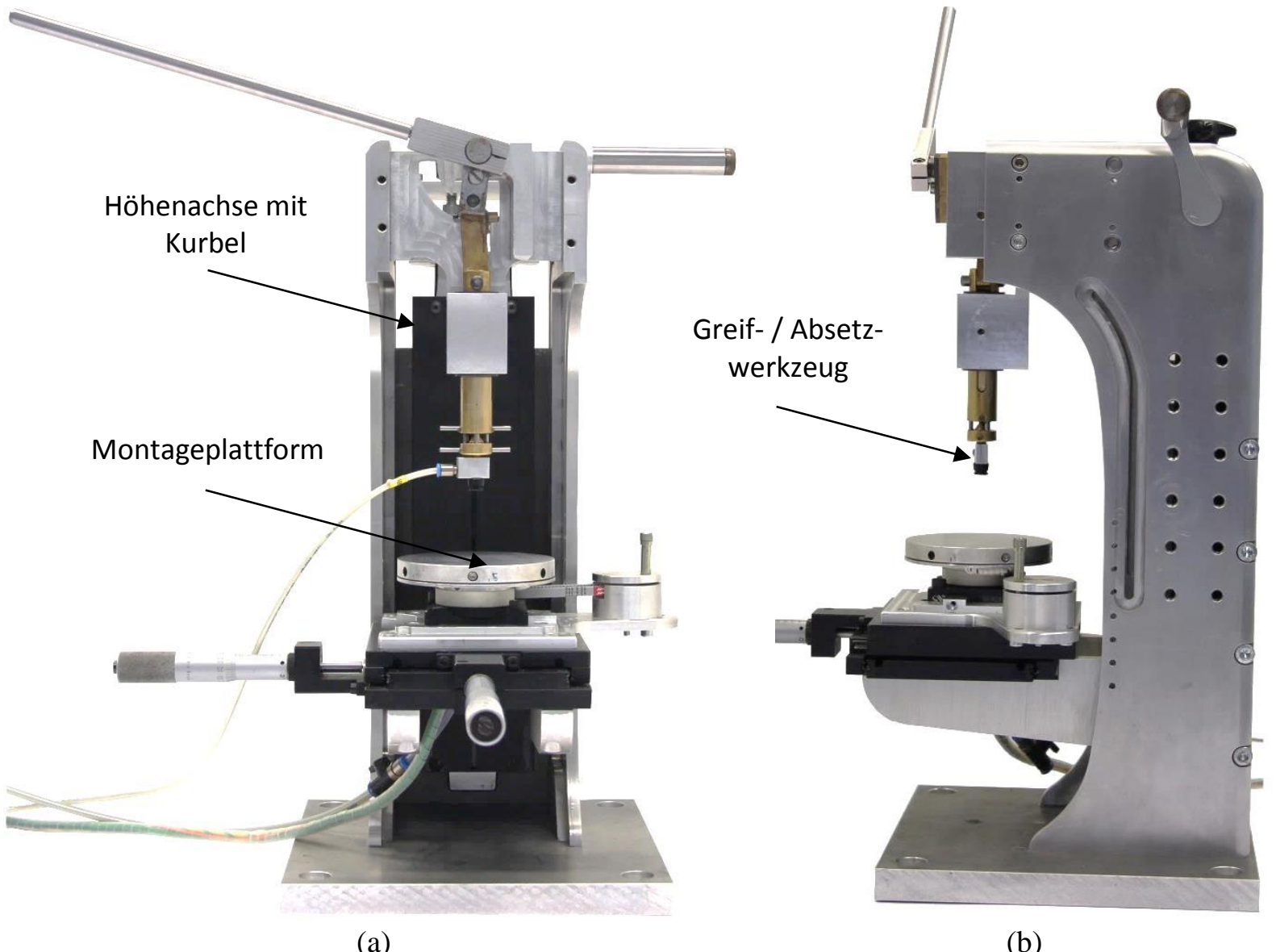

Abb. 34: Mikromontageplatz mit Fokus auf sein Gestell (a) Vorder- und (b) Seitenansicht. Seitliche Gewindebohrungen und Führungsschienen erlauben das Hinzufügen von Werkzeugen. 
Die Seitenwände des Mikromontageplatzes bestehen aus gefrästen Aluminiumplatten mit einer Dicke von $15 \mathrm{~mm}$. Ihre Form ähnelt einem C-Bogen. Der Vorteil hierbei ist, dass die über Haltearme hinzugefügten Werkzeuge seitlich weggeklappt werden können, sofern diese nicht benötigt werden. Hierdurch wird mehr Arbeitsraum an der Mikromontageplattform geboten. Die Haltearme können über Gewindebohrungen an den Seitenwänden befestigt werden. Hierzu bietet jede Seitenwand 12 Gewindebohrungen. Zusätzlich ist noch eine Führungsschiene vorhanden. An diesen Befestigungsmöglichkeiten können unterschiedlichste Werkzeuge, wie bspw. Beobachtungseinheiten, Klebstoffauftragswerkzeuge etc., angebracht werden, und eine hohe Modularität gewährleistet ist.

Ein weiterer Bestandteil des Gestelles des Mikromontageplatzes ist eine Höhenachse mit Linear-Kugelspindel, an der die Montageplattform angebracht ist. Die vorliegend eingesetzte Spindel hat einen Verfahrweg von $320 \mathrm{~mm}$ und wird von der Firma Bosch Rexroth bezogen. Zum Heben und Senken der Montageplattform über die Höhenachse ist eine Handkurbel mit mitlaufender Hülse über ein Kegelzahnrad an der Spindel angebracht. Der Verfahrweg ist ausreichend hoch, so dass neben der Annäherung an das Greif- / Absetzwerkzeug auch in der anderen Bewegungsrichtung ein ausreichend hoher Abstand gewährleistet werden kann, um Bauteile auf der Montageplattform leichter aufzulegen und entnehmen zu können. Eine Feststellbremse an der Höhenachse erlaubt eine feste Arretierung der aktuellen Höhenposition. An der Höhenachse sind zwei Gabelzinken angebracht, über die die Mikromontageplattform befestigt wird.

\subsubsection{Montageplattform am Mikromontageplatz}

Die Ausrichtung zweier Bauteile vor dem Fügevorgang erfolgt mit der Montageplattform, welche direkt an der verfahrbaren Höhenachse montiert ist. Dabei werden an die Montageplattform drei essentielle Anforderungen gestellt:

1. Ausrichtung des Zielbauteiles (Substrat) zu dem zu fügenden / abzusetzenden Bauteil

2. Fixierung des Zielbauteils

3. Bereitstellung einer Heizmöglichkeit, um bspw. Aushärtevorgänge zu beschleunigen

Um die Komponenten für die Positionierungsaufgaben an dem Mikromontageplatz zu integrieren, ist eine Lösung ausgearbeitet, welche auf engstem Raum eine große Flexibilität bietet.

Über Gabelzinken ist ein verfahrbarer Kreuztisch für die X- und Y-Ausrichtung an der Höhenachse montiert. Die Bewegung des Kreuztisches erfolgt manuell mit Mikrometerstellschrauben. Der Verfahrweg beider Achsen beträgt $50 \mathrm{~mm}$. Die eingesetzten Mikrometerstellschrauben unterliegen der DIN 863-1 (Stand April 1999), welche eine maximale Abweichung von $4 \mu \mathrm{m}$ auf $50 \mathrm{~mm}$ Weglänge vorschreibt. Um auch eine rotatorische Ausrichtung der Bauteile zu ermöglichen, wird über eine aus Aluminium gefertigte Adapterplatte ein Drehteller der Firma Leiz auf dem Kreuztisch angebracht. Dieser bietet eine stufenlos verstellbare $360^{\circ}$ Rotationsmöglichkeit und ist auf Kugeln gelagert, welche einen ruhigen und spielfreien Lauf des Drehtellers ermöglichen. Angetrieben wird der Drehteller ebenfalls manuell über einen Zahnriemen, welcher seitlich der Montageplattform mit einer Handkurbel verbunden ist. Ein direkter Kontakt des Bedieners mit dem Drehteller wird vermieden. Auf dem Drehteller wird letztendlich die Auflagefläche für den Montageprozess angebracht. Diese ist zweiteilig aufge- 
baut und besteht aus einem Halter und einem Vakuumteller mit Sauglöchern für die Fixierung des Bauteils. Abb. 35 zeigt die an der Höhenachse angebrachte Montageplattform.

Wie bereits in Kapitel 7 beschrieben, werden die Bauteile sowohl auf der Montageplattform als auch auf dem Greif- / Absetzwerkzeug mittels Vakuumtechnik fixiert. Neben einer an die Fertigung von Funktionsmodellen angepassten Entwicklung des Mikromontageplatzes stellen auch dessen Kosten eine zentrale Fragestellung dar. Da Bauteile in der Mikrosystemtechnik, wie bspw. Silizium-, Keramik- oder auch Glassubstrate, zusätzlich meist nur Gewichte im Grammbereich besitzen und somit keine großen Saugkräfte zur stabilen Fixierung erforderlich sind, kann auf einfache und kostengünstige Vakuumquellen zurückgegriffen werden. Für die Fixierung des Bauteils auf der Montageplattform wird eine bereits vorhandene Vakuumpumpe der Firma Kremer \& Kreiler verwendet. Diese erreicht einen Unterdruck von ca. $-80 \mathrm{kPa}$. Über einen Kunststoffschlauch der Firma Festo erfolgt der Anschluss der Vakuumpumpe an dem Halter des Vakuumtellers über eine Drehkupplung. Die Drehkupplung liegt in der Rotationsachse (Mittelpunkt) des Drehtellers (siehe auch Abb. 36). Somit ist eine $360^{\circ}$ Rotationsmöglichkeit des Vakuumtellers gewährleistet. Der $100 \mathrm{~mm}$ durchmessende Vakuumteller wird mit sechs M3 Schrauben an seinem Halter befestigt. Er besitzt 86 in konzentrischen Ringen angeordnete Sauglöcher mit je einem Durchmesser von 0,5 mm. Sowohl Vakuumteller als auch dessen Halter sind aus Aluminium gefertigt. Ein Austausch des Vakuumtellers gestaltet sich durch Lösen der sechs Schrauben vergleichsweise einfach.

Neben der Möglichkeit zur Ausrichtung zweier Bauteile zueinander und der sicheren Fixierung des Zielbauteils wird als eine weitere Anforderung von der Montageplattform verlangt, diese auch beheizt betreiben zu können. Hierdurch kann dem Fügeprozess thermische Energie zugeführt werden, um die Aushärtung von Klebstoff zu beschleunigen. Hierzu wird in gefrästen Nuten an der Unterseite des Halters eine Heizspirale der Firma Türk+Hillinger mit $125 \mathrm{~W}$ Leistung angebracht (siehe auch Abb. 36). Die Heizung wird mit einem ZweipunktRegelkreis gesteuert. Die entsprechende Elektronik ist in einem externen Laborgehäuse integriert. Um eine homogene Temperaturverteilung auf der Oberfläche des Vakuumtellers zu erzielen, sind sowohl die Anordnung der Heizspirale als auch das Material des Vakuumtellers wichtig. Die Anordnung der Heizspirale erfolgt direkt an dem Halter des Vakuumtellers und

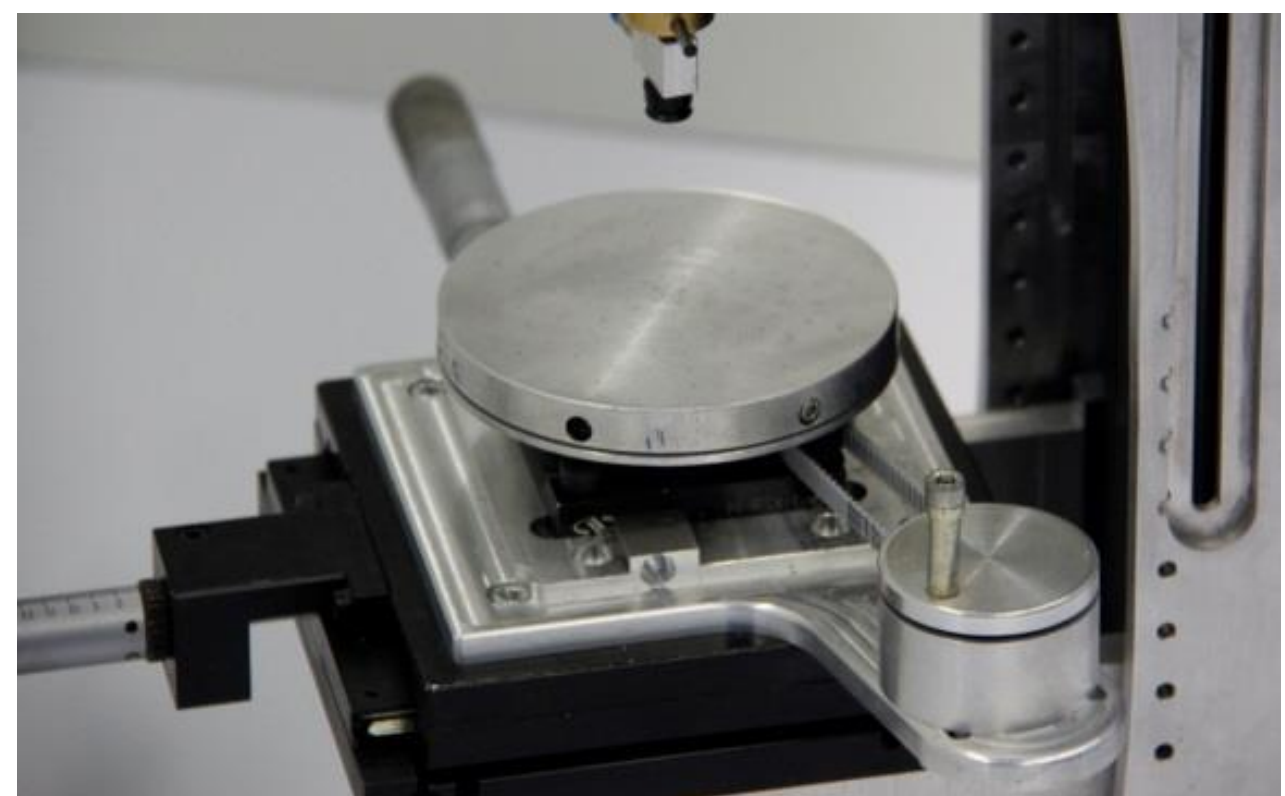

Abb. 35: Montageplattform angebracht an der Höhenachse mit Kreuztisch und Drehteller für die Ausrichtung jeweils in $\mathrm{X}$ - und Y-Richtung bzw. auch in $\theta$-Richtung. 
damit nahe seiner Oberfläche. Als Material wird Aluminium eingesetzt, welches mit $236 \mathrm{~W} / \mathrm{m} \cdot \mathrm{K}$ einen hohen Wärmeleitkoeffizienten besitzt, so dass die Wärme direkt der Oberfläche zugeführt werden kann. Gleichzeitig gilt es jedoch auch, die Wärmeverluste auf der Unterseite des Halters zu minimieren und den Wärmestrom auf die Oberfläche zu konzentrieren. Aus diesem Grund wird an der Unterseite des Halters eine Wärmeentkopplungsscheibe aus einer Glaskeramik (Vitronit ${ }^{\circledR}$ ), welche einen geringen Wärmeleitkoeffizienten von ca. $1,7 \mathrm{~W} / \mathrm{m} \cdot \mathrm{K}$ besitzt, angebracht. Abb. 37 (a) und (b) zeigen hierzu Aufnahmen mit einer Wärmebildkamera (ThermaCAM SC 500 von FLIR SYSTEMS). Deutlich erkennbar ist sowohl eine homogene Temperaturverteilung auf der Oberfläche des Vakuumtellers (siehe Abb. 37 (a)) als auch eine erfolgreiche thermische Isolation durch die Wärmeentkopplungsscheibe (siehe Abb. 37 (b)). Ein weiterer nicht unbeachtlicher Nebeneffekt ist, dass hierdurch nur eine verringerte Erwärmung der umliegenden Bestandteile der Plattform erfolgt, so dass das Verletzungsrisiko für den Operator minimiert wird.

Die in den Halter des Vakuumtellers integrierte Heizspirale mit $125 \mathrm{~W}$ Leistung ist dabei in der Lage, innerhalb von sechs Minuten den Vakuumteller auf eine maximale Temperatur von $115^{\circ} \mathrm{C}$ zu erwärmen. Hierdurch kann dem Prozess ausreichend Wärme zugeführt werden.

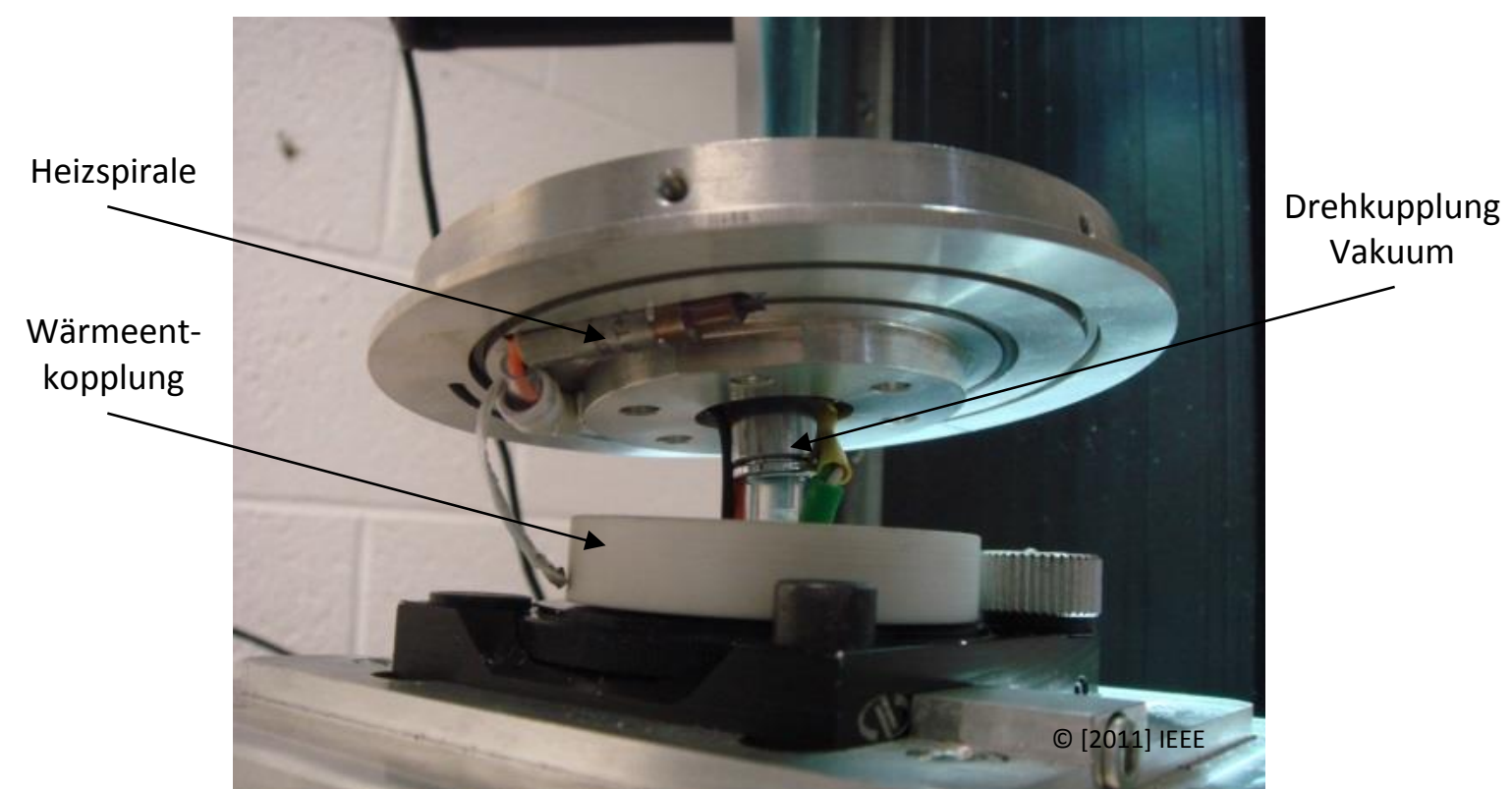

Abb. 36: Unterseite des Halters für den Vakuumteller im Detail auf dem Drehteller. Entnommen aus Kagerer et al. (2011).

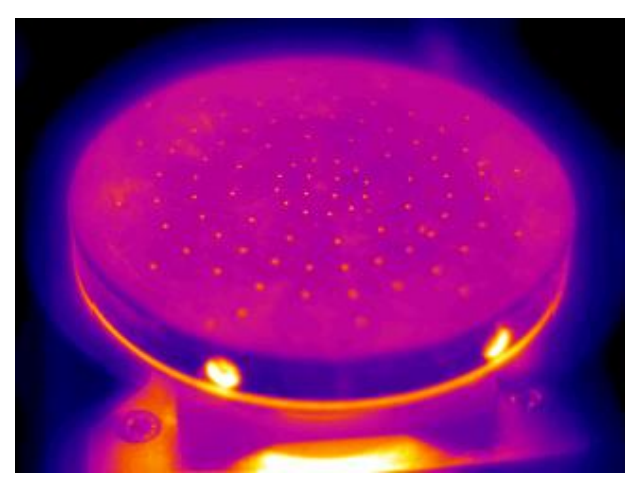

(a)

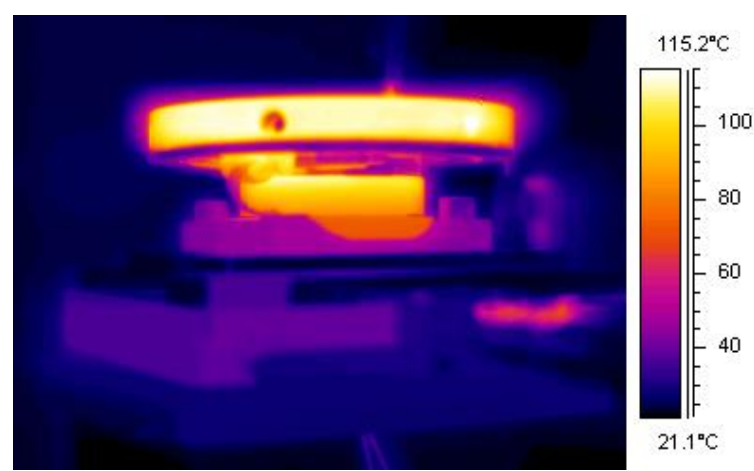

(b)

Abb. 37: Aufnahmen mit einer Wärmebildkamera der beheizten Montageplattform. (a) Homogene Temperaturverteilung auf der Oberfläche des Vakuumtellers und (b) erfolgreiche thermische Isolation mit Wärmeentkopplungsscheibe. Entnommen aus Eiler (2011). 


\subsubsection{Greif- / Absetzwerkzeug am Mikromontageplatz}

Ein weiteres essentielles Werkzeug an dem Mikromontageplatz stellt die Greif- / Absetzeinheit dar. Wie in Kapitel 7 beschrieben, wird für die kinematische Umsetzung ein zentrisches Schubkurbelgetriebe gewählt. Hierdurch wird eine Drehbewegung in eine Schubbewegung umgesetzt. Abb. 38 zeigt das realisierte Schubkurbelgetriebe am Mikromontageplatz. Um ein Bauteil auf dem Zielbauteil / Substrat auf der Montageplattform abzusetzen, wird ein Kniehebel, welcher mit dem Hebelgehäuse über eine Welle verbunden ist, heruntergedrückt. Aufgrund der kinematischen Koppelung, wird der Pleuel hierdurch abwärtsbewegt. Dieser ist wiederum mit einem Hubkolben verbunden, welcher eine Bewegung in vertikaler Richtung ausführt. Die Bestandteile des Schubkurbelgetriebes sind aus Messing gefertigt. Die Führung des Hubkolbens ist über ein schmiermittelfreies Gleitlager aus Glasfaser verstärktem Kunststoff der Firma igus gewährleistet. Am unteren Ende des Hubkolbens wird der Sauggreifer in axialer Ausrichtung angebracht.

Ein entscheidender Vorteil von Schubkurbelgetrieben ist ihre Eigenschaft, bei Verringerung des Kurbelwinkels gleichzeitig auch den Kolbenweg zu reduzieren. Für den Absetzvorgang bedeutet dies, dass zu Beginn bei großen Kurbelwinkeln die Absetzgeschwindigkeit vergleichsweise groß ist, dann jedoch zunehmend geringer wird, obwohl die Bewegung am Hebel mit der Hand in konstanter Geschwindigkeit ausgeführt wird. Hierdurch wird ein kontrolliertes und feinfühliges Absetzen von Bauteilen über das Greif- / Absetzwerkzeug in vertikaler Richtung auf das Zielbauteil / Substrat möglich. Allerdings gilt es, auf ein Überschreiten eines Kurbelwinkels von $0^{\circ}$ (Pleuel gestreckt) zu achten, da ansonsten eine erneute Aufwärtsbewegung des Kolbens durchgeführt wird. Hierzu dient eine seitlich angebrachte Schraube für die Blockade einer weiteren Drehbewegung. Ebenfalls ist in das Führungsgehäuse eine Arretierschraube angebracht, um das Greif- / Absetzwerkzeug in einer vorgegebenen vertikalen Position zu fixieren. Der ausführbare Hub des Kolbens wird nach (7.17) bei einem Kurbelradius von $29 \mathrm{~mm}$ und einer Pleuellänge von $49 \mathrm{~mm}$ zu maximal $38 \mathrm{~mm}$ berechnet. Dieser ist ausreichend, da zunächst eine Annäherung der Bauteile über die Montageplattform erfolgt.

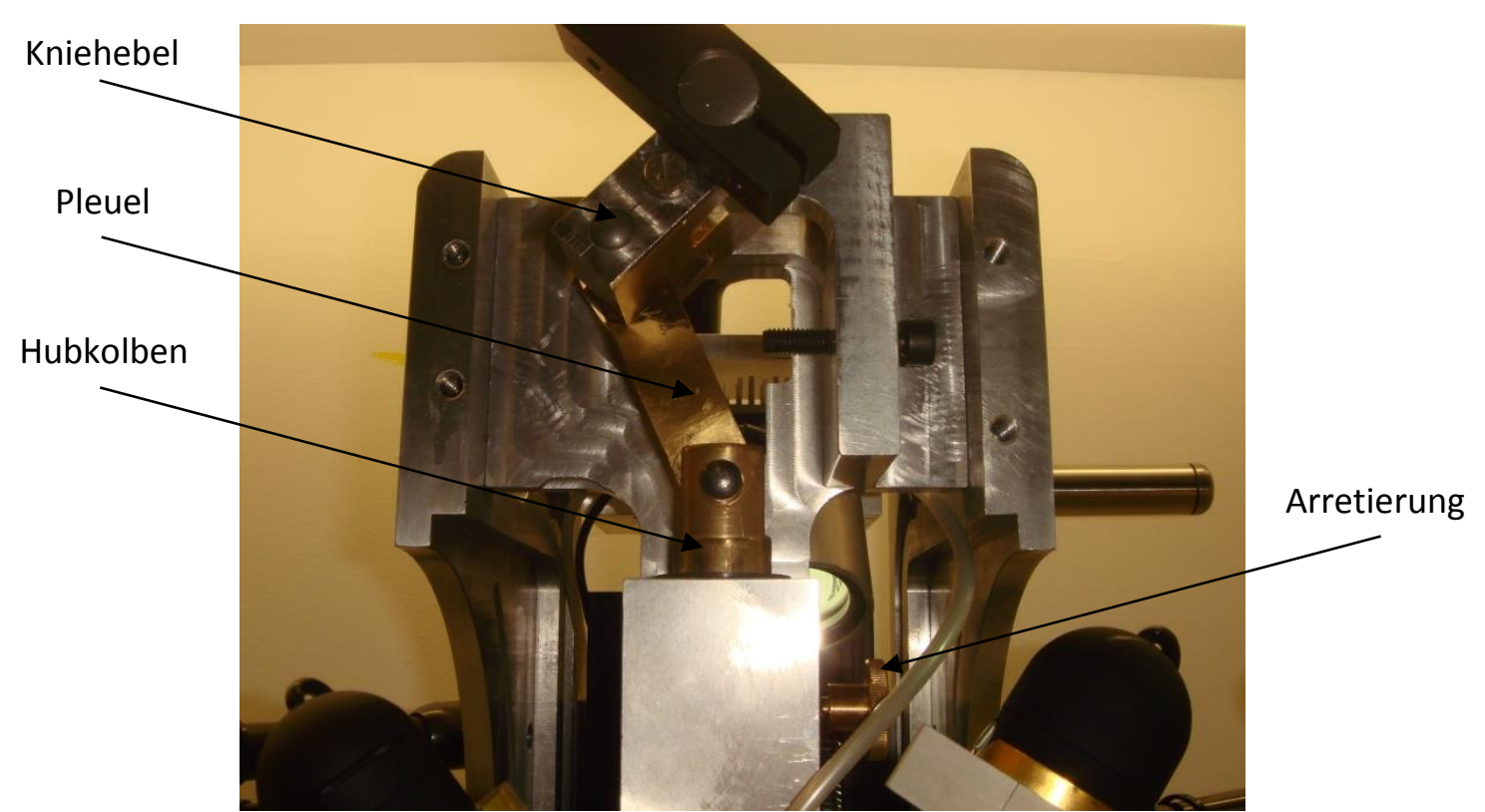

Abb. 38: Umgesetzte Kinematik des Greif- / Absetzwerkzeuges in Form eines zentrischen Schubkurbelgetriebes. 
Neben der kinematischen Umsetzung durch das Schubkurbelgetriebe ist der Sauggreifer ebenso zentraler Bestandteil. Wie in Kapitel 7 beschrieben, wird auch hier die Vakuumtechnik als Funktionsprinzip eingesetzt. Dabei zeichnen sich Vakuumsauggreifer durch eine kostengünstige Anschaffung, einem geringen Wartungsaufwand im Vergleich zu Zweibackengreifern, einer einfachen Systemintegration und einer sanften Handhabung fragiler Bauteile aus.

An dem unteren Ende des Hubkolbens werden Adapter für die Aufnahme der jeweiligen Sauggreifer benötigt. Die gefertigten Adapter besitzen eine M6 Gewindebohrung für die Befestigung an dem Kolben und eine M3 Gewindebohrung für den Anschluss der pneumatischen Versorgung über eine Schnellkupplung der Firma Festo. Die Adapter sind aus Aluminium gefertigt. Die Unterschiede liegen in der Gewindebohrung für die Aufnahme der Sauggreifer, welche meist der Größe M4 entsprechen. Dank dieser Adapterlösung ist es möglich, eine Vielzahl weiterer Sauggreifer zu verwenden. Abb. 39 zeigt hierzu beispielsweise einen Sauggreifer der Firma Festo, welcher einen Durchmesser von $10 \mathrm{~mm}$ besitzt, eine runde bzw. flache Ausführung aufweist und aus Nitrilkautschuk gefertigt ist (Modell ESS 10 SN). Dieser ist ein Standard Sauggreifer und ist für vorliegende Anwendungen, wie bspw. die Aufnahme von Substraten, vollkommen ausreichend. Der Werkstoff Nitrilkautschuk kann bis zu einer Arbeitstemperatur von $70{ }^{\circ} \mathrm{C}$ eingesetzt werden. Sind höhere Arbeitstemperaturen oder auch antistatische Eigenschaften des Saugers gefordert, kann auf Fluorkautschuk bzw. Butadien-Kautschuk zurückgegriffen werden.

Als Vakuumquelle für das Greif- / Absetzwerkzeug wird eine bereits vorhandene Vakuumsaugdüse von der Firma Festo (Modell VAD-1/4) gewählt, welche nach dem Venturi Prinzip arbeitet. Diese wird an die vorhandene Druckluftinfrastruktur der Laborumgebung angeschlossen. Mit Hilfe eines Kunststoffschlauches wird die Düse mit der Schnellkupplung des Adapters verbunden. Ist keine Druckluft in unmittelbarer Nähe vorhanden, so können auch andere Vakuumquellen eingesetzt werden. Der mit diesem Aufbau maximal erzielbare Unterdruck liegt bei ca. $-80 \mathrm{kPa}$.

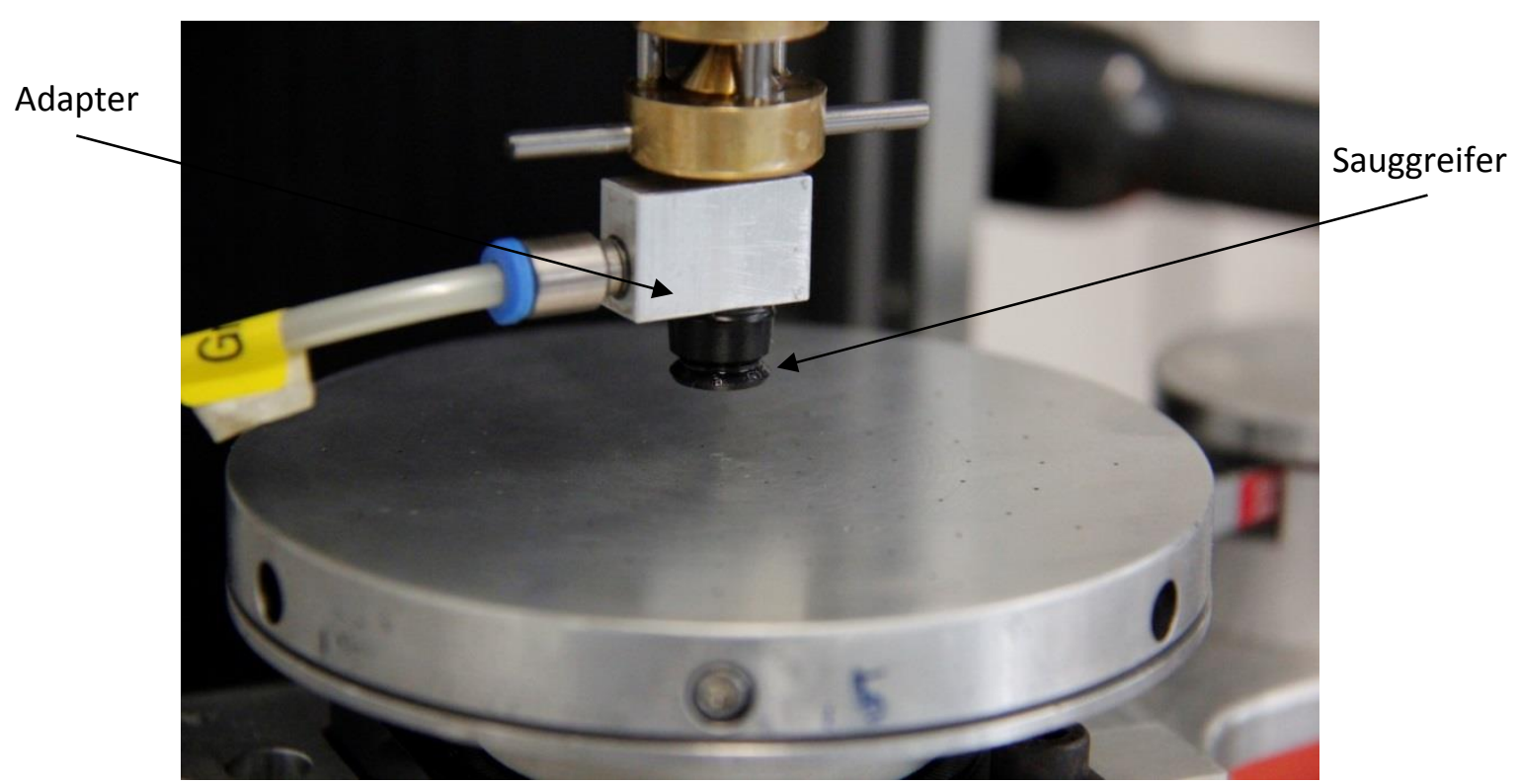

Abb. 39: Vakuumgreifer an den Hubkolben des Greif- / Absetzwerkzeuges angebracht. Mit Hilfe der Adapterlösung können unterschiedliche Sauggreifer verwendet werden. 


\subsubsection{Klebstoffauftragswerkzeug am Mikromontageplatz}

Eines der zentralen Werkzeuge am Mikromontageplatz stellt die Klebstoffauftragseinheit dar. Vor allem bei der Fügung von piezoelektrischen Wandlern ist das Kleben eines der am häufigsten eingesetzten Fügeverfahren.

Vorliegend wird ein Auftragswerkzeug realisiert, welches als Erweiterung an den vorhandenen Mikromontageplatz über einen Haltearm angebracht wird und nach dem Rakelprinzip arbeitet. Die sogenannte Rakeleinheit besteht aus drei Hauptbaugruppen (siehe Abb. 40). Die Baugruppe Rakelhalterung enthält eine Rakelaufnahme, eine Rakelklemme und eine lineare Höhenachse. Weitere Baugruppen sind der Haltearm und die Rakelklinge selbst. Alle Bauteile, welche nicht zugekauft werden, sind aus Aluminium gefertigt.

Zwischen der Rakelklemme und der Rakelaufnahme wird die Rakelklinge fixiert. In beiden Bauteilen sind Bohrungen mit spielfreien Übergangspassungen enthalten. Über zwei Passstifte werden die Rakelaufnahme und Rakelklemme relativ zueinander positioniert. Ihr endgültiger Zusammenhalt wird durch eine zentrale Schraube sichergestellt. Der Vorteil dieser Konstruktion ist, dass mit dem Öffnen einer Schraube die Rakelklinge leicht entnommen und ausgetauscht werden kann. Durch das Schließen der Schraube wird die Rakelklinge fixiert. Die zwei spielfreien Passstifte garantieren, dass sich die Rakelklemme während des SchlieBens nicht anwinkelt. Würde dieser Umstand eintreten, würde die Klemme mit nur einer Kante auf der Rakelklinge aufliegen. Das Ergebnis ist, dass die Rakelklinge nicht sicher reibschlüssig in ihrer Aufnahme befestigt.

Die Dicke der aufzutragenden Klebschicht wird über den Abstand von Rakelklinge und Substrat vorgegeben. Hierzu ist die Rakelaufnahme mit einer linearen Höhenachse, welche über eine Mikrometerschraube verfahren wird, verbunden. Über einen Adapter aus Aluminium wird die Höhenachse an einem Haltearm angebracht. Die Höhenachse wird von der Firma OptoSigma (Modell SIG-122-0075) bezogen und weist einen maximalen Verfahrweg von 6,5 $\mathrm{mm}$ bei einer minimalen Schrittweite von $3 \mu \mathrm{m}$ auf. Seitlich der Achse befindet sich ein Klemmrändel, mit dem eine Fixierung der eingestellten Position ermöglicht wird, so dass sichergestellt ist, dass sich die eingestellte Klebschichtdicke nicht verändert.

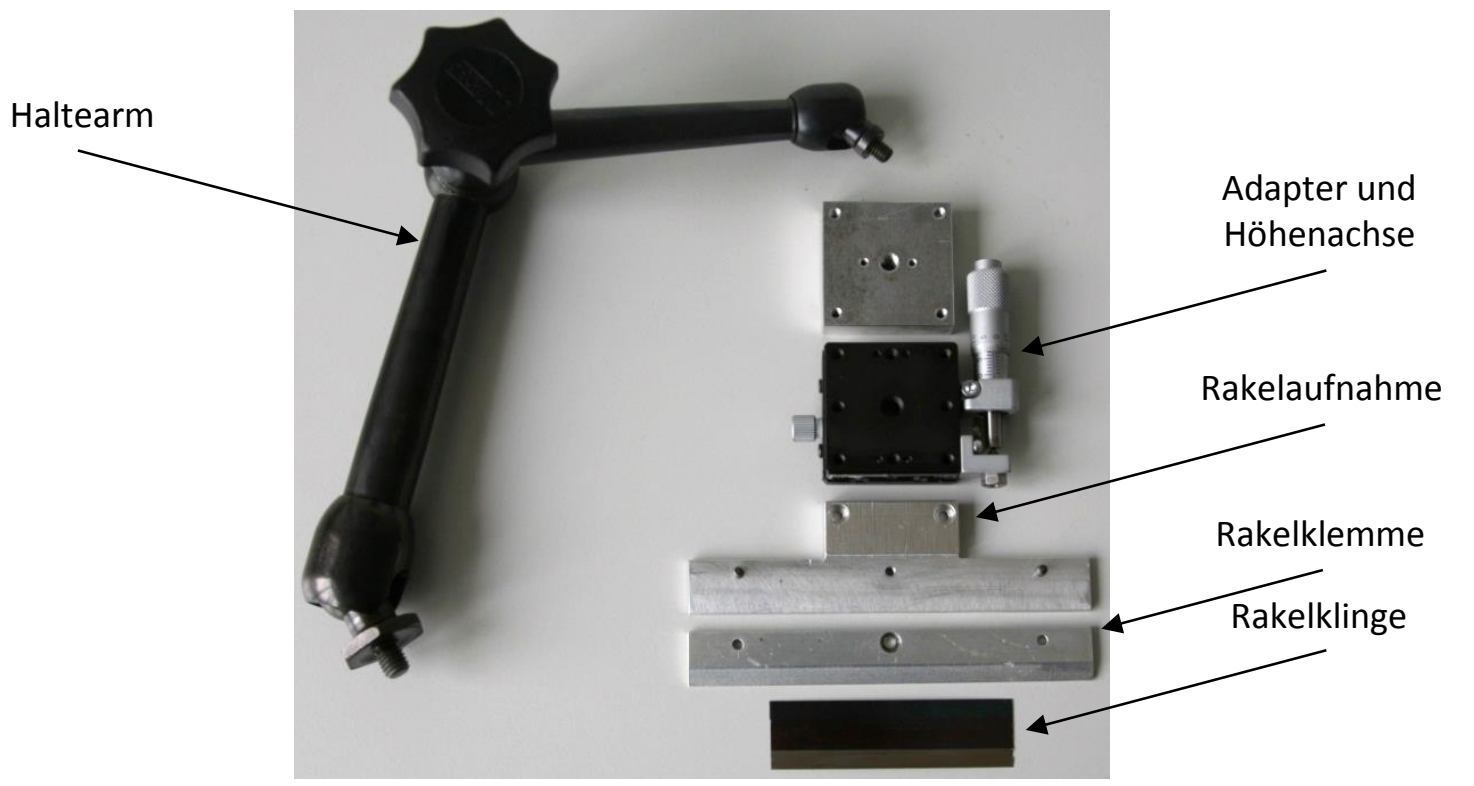

Abb. 40: Bestandteile des Klebstoffauftragswerkzeuges am Mikromontageplatz mit den Baugruppen Haltearm, Rakelhalterung (Höhenachse, Klemme und Aufnahme) und Rakelklinge. 
Als Rakel wird das Modell Flexolife der Firma Daetwyler SwissTec verwendet. Die Rakel ist $20 \mathrm{~mm}$ hoch, besitzt eine Materialstärke von 0,2 $\mathrm{mm}$ und kann auf eine beliebige Länge zugeschnitten werden. Die Rakelklinge zeichnet sich auch durch ihre Verwendbarkeit mit unterschiedlichsten Medien, wie bspw. Lösemittel, UV- und Wasserfarben, aus. Da nach erfolgreichem Klebstoffauftrag die Rakel einem Reinigungsvorgang unterzogen wird, ist die Eigenschaft der Lösemittelresistenz ideal für vorliegenden Einsatzzweck. Die Rakelkante hat einen runden Anschliff. Hiermit wird die Gefahr einer Beschädigung der Substratoberfläche vermieden und es findet keine Einschleifphase der Kante statt. Zusätzlich zeichnet sich die Rakel durch einen geringen Materialverschleiß aus. Für einen sicheren Halt der Rakel in ihrer Aufnahme ist darauf zu achten, dass sie mindestens $5 \mathrm{~mm}$ in die Aufnahme eintaucht.

Die Höhenachse wird über den Adapter an einem Haltearm der Firma Noga (Modell MG60003) montiert. Die Gesamtlänge des Armes beträgt $246 \mathrm{~mm}$. Dieser wird als Erweiterung an der Seitenwand des Mikromontageplatzes angebracht.

Abb. 41 zeigt die Rakeleinheit oberhalb der Montageplattform. Die Skala an der Mikrometerstellschraube besitzt eine Skalenteilung von $5 \mu \mathrm{m}$. Allerdings ist es hiermit nur möglich, die Höhe ohne weitere Hilfsmittel auf ca. $\pm 10 \mu \mathrm{m}$ genau einzustellen. Diese Genauigkeit reicht jedoch für vorliegenden Einsatzzweck nicht aus. Daher wird ein Messtaster der Firma Heidenhain (Modell MT12) mit einer Genauigkeitsklasse von $\pm 0,5 \mu \mathrm{m}$ in den Justierprozess integriert. Mit einem weiteren Haltearm wird dieser an dem Mikromontageplatz befestigt und oberhalb der Rakelklemme in Position gebracht. Der Verfahrweg der Höhenachse wird auf einer externen Digitalanzeige angezeigt, so dass sich die Einstellgenauigkeit der Klebschichtdicke erhöht.

An dieser Stelle wird die hohe Modularität des realisierten Mikromontageplatzes erneut sichtbar. An den Gewindebohrungen der Seitenwände können jederzeit weitere Werkzeuge über Haltearme angebracht werden. Zusammenfassend bedeutet dies für die aktuelle Konfiguration des Mikromontageplatzes, dass neben dem Klebstoffauftragswerkzeug und den Beobachtungseinheiten auch ein Messtaster für die Erhöhung von Prozessgenauigkeiten über einen Haltearm angebracht wird.

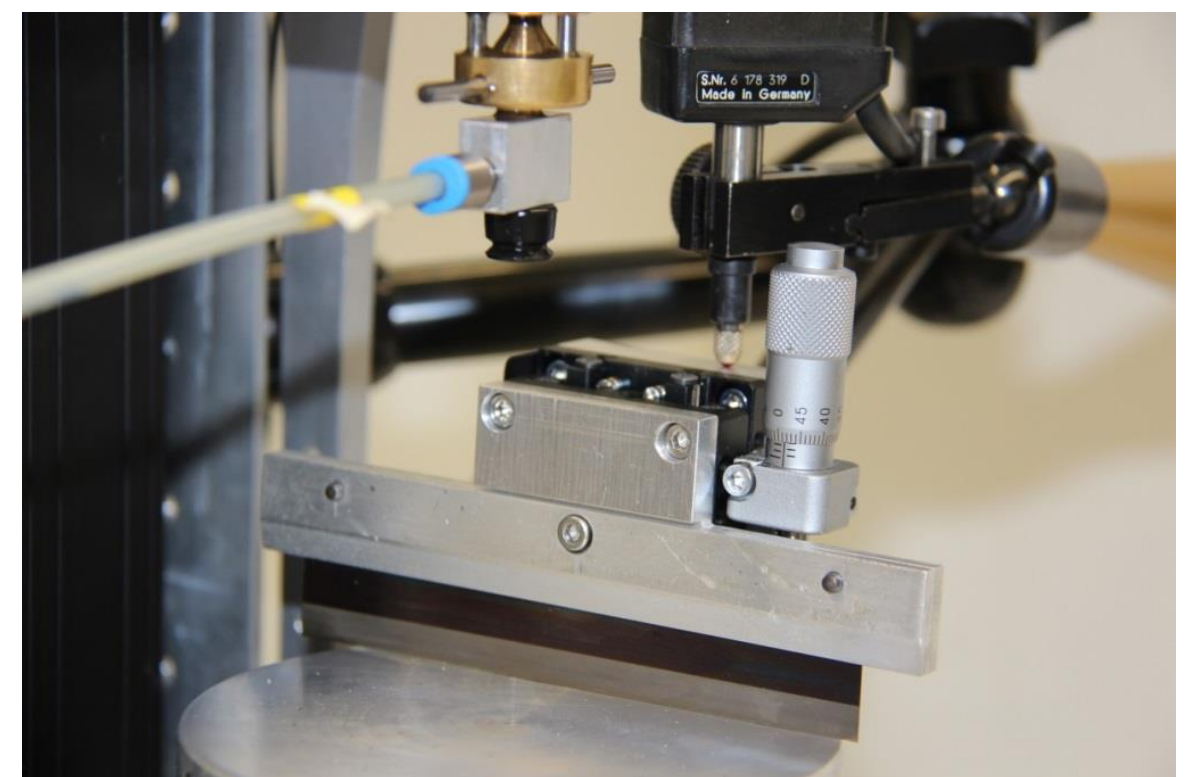

Abb. 41: Rakeleinheit auf der Montageplattform. Zur Erhöhung der Einstellgenauigkeit wird ein Messtaster in den Prozess integriert. 


\subsubsection{Gesamtaufbau des Mikromontageplatzes}

Aus der Entwicklung, der Realisierung und der Fertigung der einzelnen Werkzeuge des Mikromontageplatzes ergibt sich der Gesamtaufbau wie in Abb. 42 dargestellt. Die enthaltenen Komponenten sind hierbei:

- Rahmen inklusive Seitenwände und verfahrbarer Höhenachse

- Montageplattform mit Heizwicklung und externem Temperaturregler

- Greif- / Absetzwerkzeug mit Sauggreifer und Schubkurbelgetriebe

- Klebstoffauftragswerkzeug montiert über Haltearm

- Zwei Videomikroskope montiert über Haltearme inklusive PC

- Messtaster und Digitalanzeige zur Erhöhung der Einstellgenauigkeiten

Die Videomikroskope werden von der Firma dnt (Modell DigiMicro2.0 Scale) bezogen. Diese bieten eine stufenlos bis zu 200fach einstellbare Vergrößerung und einen Bildsensor mit zwei Megapixel.

Die Aufgabe, in einem Werkzeug alle benötigten Funktionen für die Fügung von zwei Bauteilen an einem Ort bzw. in einem Werkzeug zur Verfügung zu stellen, ist damit erfolgreich umgesetzt. Es werden dadurch Um- bzw. Neujustageprozesse vermieden, so dass das Risiko von Prozessfehlern minimiert wird. Der Aufbau erweist sich als intuitiv bedienbar und kostengünstig. Es sind keine motorbetriebenen Achsen für Relativpositionierungsaufgaben notwendig und der Klebstoffauftrag auf Substraten erfolgt mit Hilfe des Rakelprinzips ganzflächig. Das umgesetzte Schubkurbelgetriebe erlaubt ein feinfühliges Absetzen von Bauteilen mit dem Greif- / Absetzwerkzeug; auch zeichnet sich der Mikromontageplatz durch eine hohe Modularität aus.

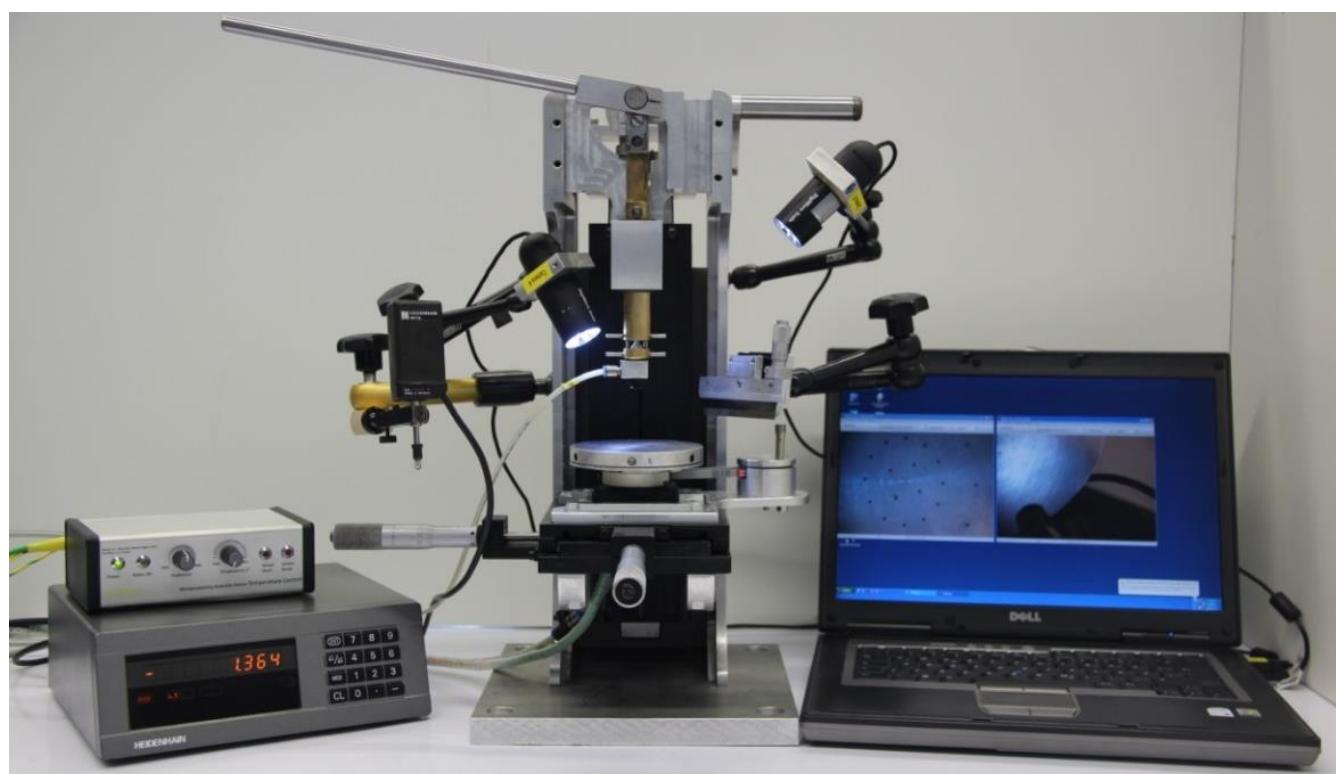

Abb. 42: Gesamtaufbau des realisierten Mikromontageplatzes (Vakuumquellen nicht abgebildet). Dieser zeichnet sich sowohl durch eine intuitive Bedienbarkeit und eine hohe Modularität als auch durch eine platzsparende und kostengünstige Bauweise aus. 


\subsubsection{Anwendung des Mikromontageplatzes}

\subsubsection{Absetzen von Katalysatoren in einem Mikrotriebwerk}

Eine zentrale Aufgabe, welche mit dem vorliegenden Mikromontageplatz gelöst wird, ist das Absetzen von Bauteilen an definierten Positionen. Das Hauptaugenmerk bei seiner Entwicklung liegt auf dem Absetzen von planaren Substraten. Diese Aufgabe gestaltet sich auf Grund der makroskopischen Dimensionen vergleichsweise einfach. Um dennoch seine Funktionsfähigkeit bzw. Tauglichkeit auch für die Montage mikrostrukturierter Bauteile aufzuzeigen, wird ein Katalysator in die Brennkammer eines Mikrotriebwerkes montiert (siehe Abb. 43).

Die Katalysatoren bestehen aus einer Metalllegierung, haben eine Fläche von 4 x 1,5 mm² und sind $300 \mu \mathrm{m}$ dick. Ihre Oberfläche ist mit Kanälen, welche $200 \mu \mathrm{m}$ breit sind, strukturiert. Die Montage des Katalysators erfolgt in einer abladierten Brennkammer auf einem Siliziumchip, welche nahezu die gleichen Dimensionen einnimmt, wie der Katalysator selbst. Anhand der Form des Katalysators wird ersichtlich, dass nur ein Sauggreifer als Werkzeug für die Montage verwendet werden kann. Zweibackengreifer besitzen für diese Aufgabe keine Möglichkeit, den Katalysator, ohne dabei die beschichteten Kanalstege zu berühren, aufzunehmen. Dies könnte zu einer Beschädigung der Beschichtung an diesen Stellen führen. Mit Hilfe der Adapterlösung an dem Greif- / Absetzwerkzeug ist es indes möglich, einen Sauggreifer mit 1,6 mm Durchmesser zu verwenden. Auch wenn wie vorliegend der Sauggreifer breiter als das Bauteil selbst ist, liegt dennoch eine ausreichend hohe Ansaugkraft vor.

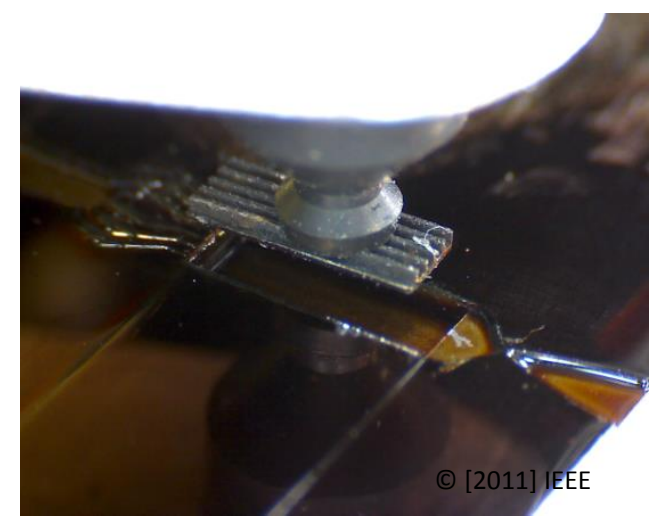

(a)

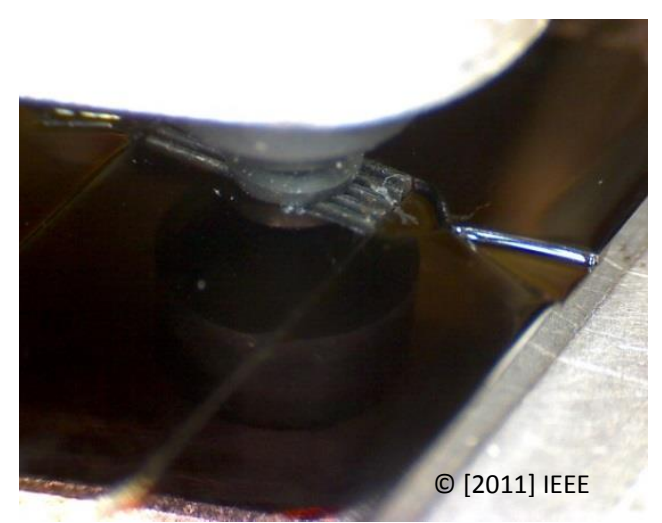

(c)

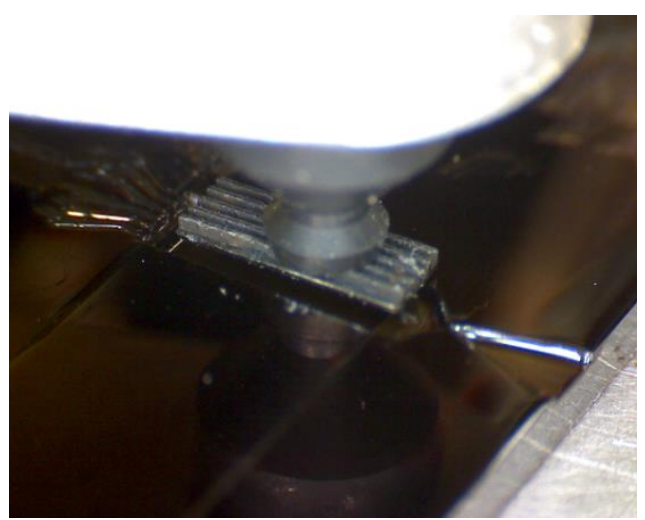

(b)

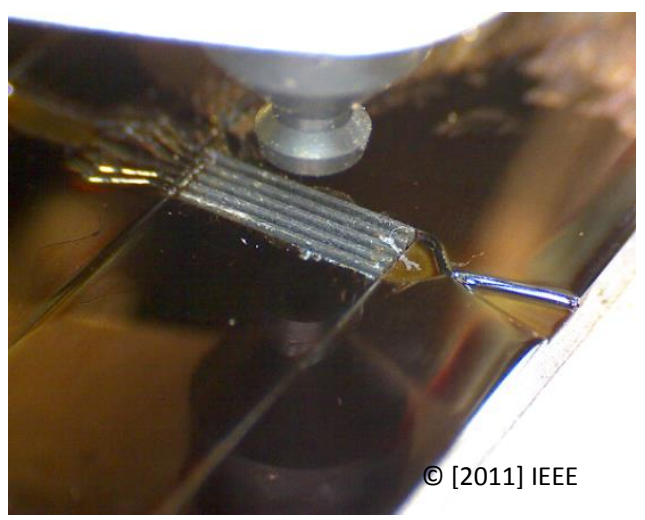

(d)

Abb. 43: Montageschritte für einen Katalysator mit Mikrostrukturen in eine Brennkammer eines Mikrotriebwerkes. (a) Aufnahme, (b) Positionieren, (c) Absetzen und (d) Lösen des Vakuums. (a), (c) und (d) entnommen aus Kagerer et al. (2011). 
Die Montageschritte sind dabei im Einzelnen:

1. Aufnahme des abzusetzenden Bauteils (Katalysator) mit dem Sauggreifer

2. Fixierung des Zielbauteils (Mikrotriebwerk) auf der Montageplattform und manuelle grobe Ausrichtung zu dem abzusetzenden Bauteil

3. Annäherung des abzusetzenden Bauteils bis ein geringer vertikaler Abstand zu dem Zielbauteil besteht

4. Positionierung beider Videomikroskope

5. Ausrichtung des Zielbauteils mit der Mikromontageplattform

6. Optional: Sofern eine Relativpositionierung vorgenommen wird, wird die Montageplattform über die Mikrometerstellschrauben in X- und Y-Richtung verfahren. Auch hier wird zur Erhöhung der Positioniergenauigkeit der Messtaster verwendet

7. Absetzen des Bauteils auf dem Zielbauteil und Vakuum lösen

Unter Beachtung dieser Schrittfolge werden mit dem Mikromontageplatz alle Montageaufgaben durchgeführt. Durch das Hinzufügen des Messtasters wird zudem die Positioniergenauigkeit erhöht. Es wird an dieser Stelle zusammenfassend festgehalten, dass die Montage eines Katalysators mit Mikrostrukturen in einer Brennkammer erfolgreich durchgeführt worden ist.

\subsubsection{Auftrag von Klebstoff}

Eine weitere zentrale Aufgabe, welche durch den Mikromontageplatz gelöst wird, ist der ganzflächige Auftrag von Klebstoff auf Substraten. Abb. 44 zeigt hierzu die Prozessfolge für den Klebstoffauftrag auf einer $100 \mu \mathrm{m}$ dicken Borosilikatglasmembran auf Silizium. Die Auftragsfläche entspricht dabei einem geviertelten 4 Zoll Wafer (ca. $20 \mathrm{~cm}^{2}$ ).

Als Vorbereitung für den Klebstoffauftrag ist vorab der Vakuumteller orthogonal zu der Rakelrichtung auszurichten. Hierzu wird der Messtaster auf dem Vakuumteller aufgesetzt. Anschließend wird der Vakuumteller unter dem Messtaster verfahren und etwaige Höhendifferenzen an der externen Anzeige abgelesen. Da der Vakuumteller seitlich über Schrauben an seinem Halter befestigt ist, kann eine orthogonale Ausrichtung eingestellt werden. Des Weiteren sind die Vakuumsauglöcher um das Bauteil mit einer Folie zu schützen, so dass diese nicht durch Klebstoff verstopft werden.

Nach der Prozessvorbereitung ergeben sich folgende Schritte für den Klebstoffauftrag:

1. Fixierung des Substrates auf der Montageplattform und Positionierung der Rakeleinheit oberhalb des Substrates mit angesetztem Messtaster

2. Öffnen der Klemmschraube, so dass die Rakelklinge in Kontakt mit dem Substrat ist

3. Einstellen der Schichtdicke und Fixierung der Position mit dem Klemmrändel

4. Startposition des Rakelprozesses anfahren

5. Klebstoff grob mit der Hand vorverteilen 
6. Ununterbrochenes Verfahren der Montageplattform unterhalb der Rakelklinge

7. Klebstoffauftragswerkzeug mit Haltearm seitlich wegklappen und reinigen

8. Optional: Sofern ein Fügevorgang gefordert ist, wird das abzusetzende Bauteil über den Sauggreifer aufgenommen. Mit Hilfe der Montageplattform werden beide Bauteile zueinander ausgerichtet

Mit vorliegendem Prozessflow können Klebstoffschichten vor allem homogen und ganzflächig auf planaren Substraten aufgebracht werden. Es wird an dieser Stelle festgehalten, dass der Klebstoffauftrag auf Substraten erfolgreich durchgeführt werden kann.

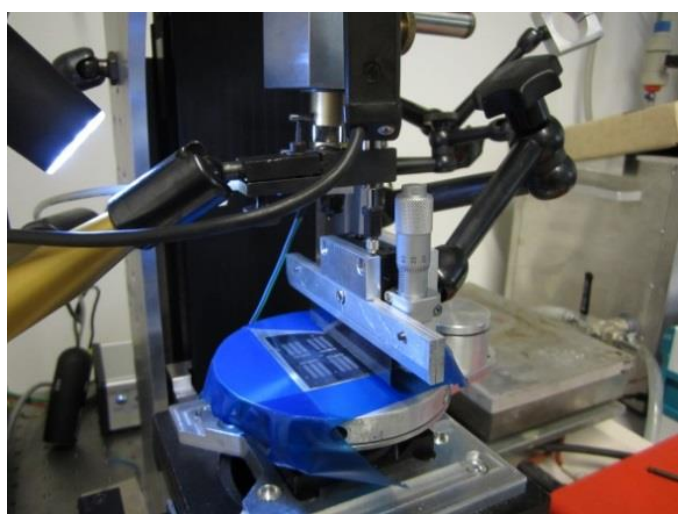

(a)

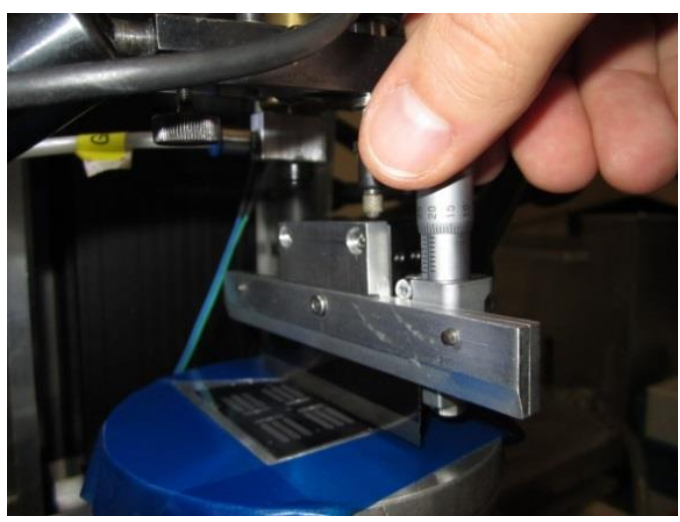

(c)

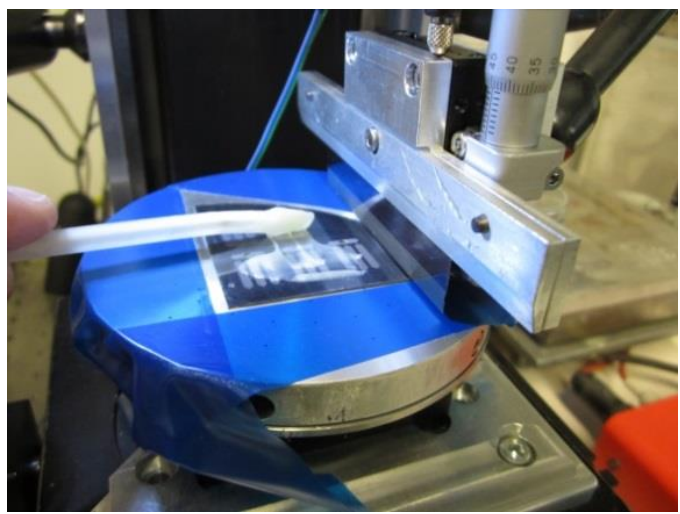

(e)

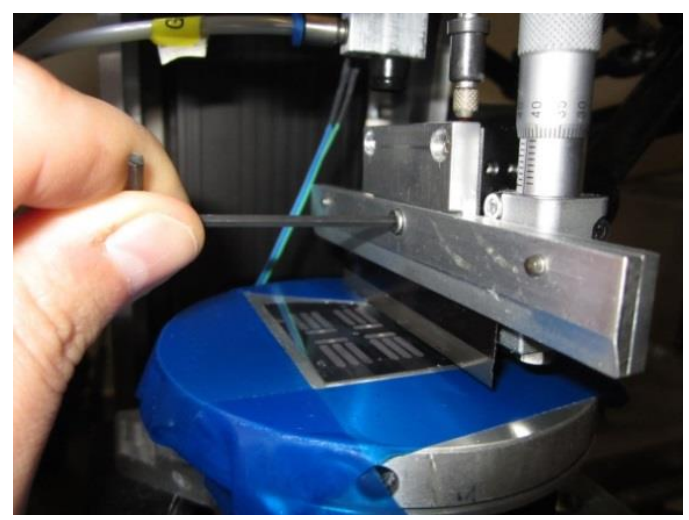

(b)

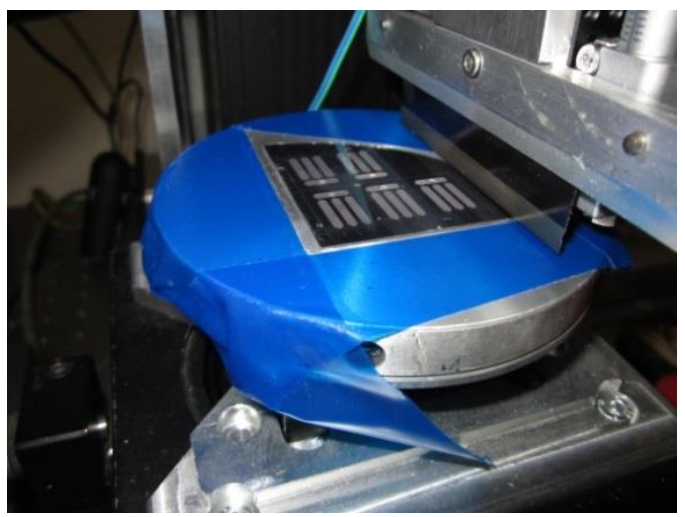

(d)

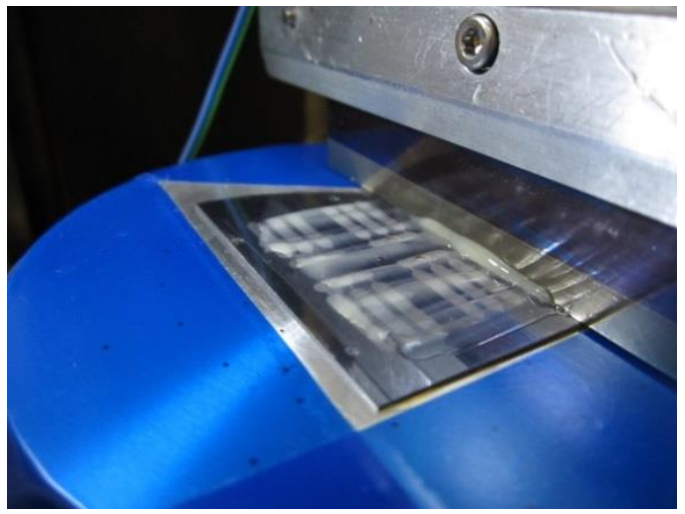

(f)

Abb. 44: Prozessflow für den Klebstoffauftrag. (a) Positionierung der Rakeleinheit, (b) Rakelklinge in Kontakt mit dem Substrat bringen, (c) Schichtdicke einstellen, (d) Startposition anfahren, (e) Klebstoff manuell auftragen und (f) Rakelprozess. Entnommen aus Hüdig (2014). 


\subsection{Zusammenfassende Betrachtung der Realisierung}

In der Fertigung von Funktionsmodellen in geringen Stückzahlen für die Mikrosystemtechnik werden sowohl Mikrostrukturierungs- als auch Mikromontageoperationen verlangt. Nachfolgend wird hierzu die in vorliegender Arbeit für die erfolgreiche Lösung dieser Aufgaben durchgeführte Realisierung zusammengefasst.

Für den Laserstrahlmaterialabtrag werden zwei im Nanosekundenbereich gepulste Laserstrahlquellen in Verbindung mit Galvanometerscannern eingesetzt. Beide Bearbeitungssysteme (siehe Abb. 28) verfügen über motorbetriebene Achsen, mit denen Werkstücke sowohl horizontal als auch vertikal im Arbeitsraum positioniert werden können. In Kapitel 7 wird beschrieben, dass der Einfluss von Laserstrahlquellen unabhängigen Parametern (Fokusposition, Vorschub und Linienabstand) auf den Materialabtrag untersucht wird. Beide Systeme verfügen hierzu über die notwendigen Gegebenheiten, um diese Untersuchungen durchzuführen. Der Vorschub ist in ausreichender Bandbreite variierbar, Schnitt- / Abtragsmuster mit versetzten Linien können gefertigt werden und es steht bei beiden Systemen ein Fokusfindungsprozess zur Verfügung. Für das Trennschleifen von Silizium mit einer Wafersäge wird ein Sägeblatt mit einer metallischen Nickelbindung und geringem Schleifkorn gewählt. Hierdurch werden sowohl eine vergleichsweise hohe Blattstandzeit als auch feine Schnitte mit geringem Chipping erzielt. Die Siliziumsubstrate werden in vorliegender Arbeit nicht auf standardisierten Blue Tapes, sondern auf UV Licht empfindlichen Sägefolien fixiert. Der Vorteil ist, dass Substrate hierauf einer stärkeren Haftkraft ausgesetzt sind und mögliche Bewegungen des Substrates während des Schleifprozess vermieden werden. Zudem erfolgt eine beschädigungsfreie Lösung der Substrate von der Sägefolie mittels UV Belichtung.

Für die Fügung von zwei Bauteilen über eine Klebschicht ist ein Mikromontageplatz realisiert. Dieser besteht in seinen Hauptbestandteilen aus einem Gestell, einem Greif- / Absetzwerkzeug, einem Klebstoffauftragswerkzeug, einer Montageplattform und weiterer Peripherie. Der Mikromontageplatz ist modular aufgebaut, so dass über Haltearme weitere Werkzeuge integriert werden. Das abzusetzende Bauteil wird über einen Vakuumsauggreifer aufgenommen. Mittels einer Adapterlösung ist es möglich, bauteil- und prozessspezifische Sauggreifer anzubringen und rasch auszutauschen. Die eingesetzte Kinematik des Greif- / Absetzwerkzeuges entspricht dabei einem zentrischen Schubkurbelgetriebe. Die Ausrichtung des Zielbauteils wird mit einer Montageplattform in X-, Y- und $\theta$-Richtung manuell vorgenommen. Die Fixierung des Zielbauteils erfolgt auch mit Vakuum. Des Weiteren steht hier auch eine Heizmöglichkeit zur Verfügung, so dass eine maximale Betriebstemperatur der Montageplattform von $115^{\circ} \mathrm{C}$ nach einer Aufheizzeit von sechs Minuten erzielt wird. Eine thermische Isolierung ist durch eine Wärmeentkopplungsscheibe vorgenommen. Um homogene und ganzflächige Klebstoffschichten auf planaren Substraten zu erzielen, ist ein Klebstoffauftragswerkzeug realisiert. Dieses besteht aus einer Rakelaufnahme, einer Rakelklemme, einer linearen Höhenachse, einer Rakelklinge und einem Haltearm. Das Arbeitsprinzip entspricht dabei dem Abziehen von Klebstoff auf dem Zielbauteil mit einer Rakelklinge. Mit einer Mikrometerstellschraube an einer Linearachse wird hierbei die Klebschichtdicke als Folge des Abstandes zwischen Rakelklinge und Substrat eingestellt. Für die Visualisierung des Montagevorganges werden zwei Videomikroskope eingesetzt. Zur Erhöhung der Prozessgenauigkeiten wird ein Messtaster verwendet. Die Realisierung des Mikromontageplatzes zeigt, dass in einem Werkzeug alle benötigten Funktionen für die Fügung von zwei Bauteilen an einem Ort zur Verfügung gestellt werden. Um- bzw. Neujustageprozesse werden vermieden, wodurch mögliche Prozessfehler reduziert werden. 


\section{Experimente zur Mikrostrukturierung und zur Mikromontage}

Nach der Beschreibung des eigenen Ansatzes und der Realisierung sowohl für die Mikrostrukturierung als auch für die Mikromontage werden die notwendigen Experimente zu deren Bestätigung durchgeführt.

\subsection{Experiment 1: Laserstrahlschneiden in Silizium}

\subsubsection{Beschreibung des Messverfahrens}

In diesem Experiment gilt es aufzuzeigen, welchen Einfluss die Laserstrahlquellen unabhängigen Parameter auf das Laserstrahlschneiden in Silizium, bezogen auf die Anzahl an notwendigen Überfahrungen unter Beachtung der Prozesszeit für einen Ausschnitt, besitzen. Im Detail sind dies die Fokusposition, die Vorschubgeschwindigkeit und der Linienabstand.

Folgende Hypothese soll an dieser Stelle mit dem Experiment bestätigt werden: Es ist möglich, mit einem leistungsschwachen UV Lasersystem $\left(\mathrm{P}_{\text {Mittel }}=2 \mathrm{~W}\right)$ mit maximal 10 Laserspotüberfahrungen - unter Beachtung der Prozesszeit - Ausschnitte in $300 \mu \mathrm{m}$ dickem Silizium zu erreichen, indem die Laserstrahlquellen unabhängigen Parameter optimal aufeinander abgestimmt werden.

Es werden in $300 \mu \mathrm{m}$ dicken Siliziumchips Durchgangslöcher mit einem Durchmesser von 1,6 mm geschnitten. Die Wahl dieser Form ist dadurch begründet, dass diese Durchgänge sowohl für die elektrische Kontaktierung als VIAs (vertical interconnect access) als auch für den fluidischen Anschluss von Mikrosystemen eingesetzt werden können. Es wird das UV Lasersystem Gator (siehe Abb. 28 (a)) mit einer fest eingestellten Pulsfrequenz von $10 \mathrm{kHz}$ und einer mittleren Leistung von $2 \mathrm{~W}$ verwendet. In Liess (2006) wird für vorliegendes Lasersystem bereits der Bearbeitungsdurchmesser in Silizium zu $15 \mu \mathrm{m}$ bestimmt. Ungeklärt ist bislang jedoch der Einfluss der Laserstrahlquellen unabhängigen Parameter auf die Effizienz bei Ausschnitten in Substraten. Bei der Dimensionierung der Schneidformen wird der Bearbeitungsdurchmesser $d_{W}$ und der sogenannte Wobbelradius $r_{\text {Wobbel }}$ von $10 \mu \mathrm{m}$, eine zusätzliche kreisförmige Bewegung des Laserstrahls auf seinem ursprünglichen Verfahrweg, berücksichtigt. Die Dimension der Schneidform $d_{\text {Shape }}$ berechnet sich nach (10.1). $d_{\text {Soll }}$ stellt das geforderte Sollmaß der Form auf dem Substrat dar. Die Anzahl der notwendigen Überfahrungen und die Prozesszeit werden manuell aufgenommen. Es wird der Mittelwert aus mindestens drei Messproben pro Messpunkt gebildet.

$$
d_{\text {Shape }}=d_{\text {Soll }}-\left(d_{W}+2 \cdot r_{\text {Wobbel }}\right)
$$

\subsubsection{Experiment zur Messung der Vorteile}

$\mathrm{Zu}$ Beginn des Experimentes wird ein Parametersatz gewählt, welcher sukzessive optimiert wird. Hierbei beträgt der Vorschub $10 \mathrm{~mm} / \mathrm{s}$ (PÜ=97,1\%) und der Linienabstand $10 \mu \mathrm{m}$ (SÜ=71,4\%). Es werden zwei konzentrische Kreise als Schneidformen gewählt. Als erster Parameter wird die Fokusposition variiert und deren Einfluss auf das Abtragsergebnis aufgezeigt. Ist eine optimale Fokusposition gefunden, wird die Vorschubgeschwindigkeit und im Anschluss der Linienabstand optimiert. Schritt für Schritt wird ein Parameter nach dem ande- 
ren optimiert. Nach der Abstimmung der Laserstrahlquellen unabhängigen Parameter wird zudem untersucht, ob mehr als zwei Schneidformen die Anzahl an notwendigen Überfahrungen für einen Ausschnitt reduzieren. Die Ausschnitte in den Siliziumchips erfolgen im Überhang, so dass der darunterliegende Auflageteller des Lasersystems bei erfolgreichem Ausschnitt nicht beschädigt wird. Das Experiment findet ohne Prozessgas an Luft statt. Die Schneidformen werden mit Hilfe der Steuerungssoftware des Galvanometerscanners erstellt.

Mit dem in Kapitel 9 beschriebenen Fokusfindungsprozess ist es möglich, den Fokus in $50 \mu \mathrm{m}$ großen Schritten durch den $300 \mu \mathrm{m}$ dicken Siliziumchip zu legen. Dabei stellt die Fokusposition $0 \mu \mathrm{m}$ die Werkstückmitte, die Position $150 \mu \mathrm{m}$ die Werkstückoberseite und die Position $-150 \mu \mathrm{m}$ die Werkstückunterseite dar. Zwei weit entfernte Positionen, $150 \mu \mathrm{m}$ oberhalb der Oberfläche und $150 \mu \mathrm{m}$ unterhalb der Unterseite stellen die Extremwerte dar. Der Fokus wird konstant gehalten. Abb. 45 (a) zeigt die Anzahl an notwendigen Überfahrungen für den Ausschnitt im Silizium. Dabei zeigt sich, dass die geringste Anzahl an Überfahrungen in einem Bereich von $\pm 50 \mu \mathrm{m}$ um eine mittig im Werkstück gelegene Fokusposition benötigt wird. Es werden 60 Überfahrungen für den Ausschnitt verlangt. Dies erklärt sich durch eine Bearbeitung im Nahfeld (Grenze Rayleigh Länge) des Laserstrahls, so dass eine symmetrische Intensitätsverteilung um die Mitte des Werkstückes resultiert. Je weiter der Fokus von der Mitte des Werkstückes entfernt ist, desto mehr Überfahrungen werden benötigt. Ergänzend wird an dieser Stelle der Flankenwinkel $\alpha$ des Ausschnittes als weiteres Bewertungskriterium hinzugezogen. Dieser berechnet sich nach (10.2) unter Berücksichtigung des Eintrittsund Austrittsdurchmessers $\mathrm{d}_{\text {Eintritt }} / \mathrm{d}_{\text {Austritt }}$ und der Materialdicke $\mathrm{T}$.

$$
\alpha=\arctan \left(\frac{2 \cdot T}{d_{\text {Eintritt }}-d_{\text {Austritt }}}\right)
$$

Abb. 45 (b) zeigt, dass die steilsten Flanken bei einem mittig im Werkstück gelegenen Fokus auftreten. Es wird eine nahezu senkrechte Flanke unter einem Winkel von 87,4 erzielt. Es wird an dieser Stelle das Zwischenfazit gezogen, dass die optimale Fokusposition für das Laserstrahlschneiden in der Mitte des Werkstückes liegt.

Um einen höheren Materialabtrag pro Überfahrung zu erhalten, wird die Vorschubgeschwindigkeit reduziert, um dadurch den Pulsüberlapp nach (7.11) zu erhöhen. Es wird mehr Energie an nahezu gleicher Stelle im Werkstück eingebracht. Das Ergebnis ist, dass dadurch mehr

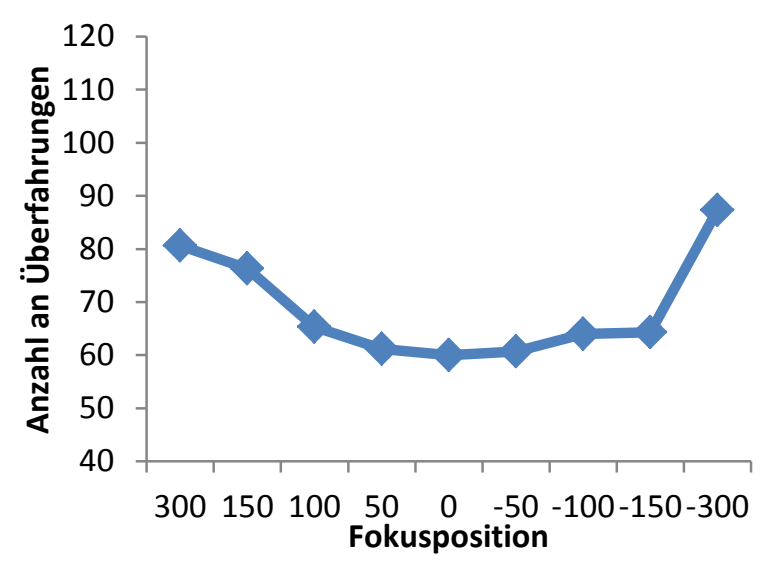

(a)

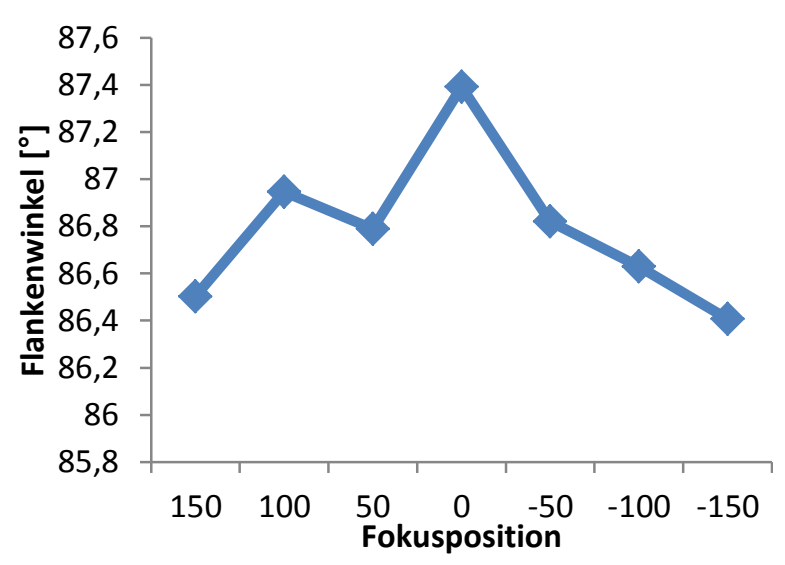

(b)

Abb. 45: Einfluss der Fokusposition auf den Materialabtrag. (a) Anzahl an notwendigen Überfahrungen für einen Ausschnitt und (b) resultierender Flankenwinkel. Messwerte entnommen aus Kagerer, Irlinger und Lueth (2011). 
Material pro Überfahrung abgetragen wird. Allerdings gilt es, auf die Prozesszeit zu achten. Der Grundgedanke vorliegender Arbeit ist es, nämlich nicht möglichst viel Material in wenig Überfahrungen abzutragen, sondern in möglichst kurzer Zeit. Abb. 46 zeigt hierzu das Abtragsverhalten unter der Variation des Vorschubes auf. Je niedriger der Vorschub bzw. je höher hieraus folgernd der Pulsüberlapp ist, desto weniger Überfahrungen werden für einen Ausschnitt benötigt. Dies zeigt auch Abb. 47. Die kürzeste Prozesszeit von $64 \mathrm{~s}$ wird bei einem Vorschub von $2 \mathrm{~mm} / \mathrm{s}$ (PÜ=99,4 \%) erzielt. Es findet ein optimales Zusammenspiel zwischen Geschwindigkeit und Abtragsrate statt. Es werden 12 Überfahrungen $(25 \mu \mathrm{m} / \mathrm{Scan})$ für einen Ausschnitt benötigt. Es wird daher das Zwischenfazit gezogen, dass eine Reduzierung des Vorschubes in einem gewissen Bereich den Materialabtrag erhöht bei gleichzeitiger Reduzierung der Prozesszeit aufgrund einer geringeren Anzahl an notwendigen Überfahrungen.

Um dem Abschwächen des Laserstrahls in der schmalen Bearbeitungsfuge entgegenzuwirken, besteht die Möglichkeit, diese Fuge zu verbreitern, damit das verdampfte Material mehr Platz findet, um zu entweichen. Je größer der Linienabstand ist, desto geringer ist nach (7.12) der resultierende Spurüberlapp. Es werden bislang zwei konzentrische Kreise mit einem Linienabstand von $10 \mu \mathrm{m}$ als Schneidform eingesetzt. Abb. 48 (a) zeigt hierzu das Abtragsverhalten unter der Variation des Linienabstandes auf. Es zeigt sich, je größer der Linienabstand respektive je breiter die Schnittfuge ist, desto weniger Überfahrungen werden für einen Ausschnitt benötigt. Gleichzeitig ist damit eine Reduzierung der Prozesszeit verbunden, da der Durchmesser der innenliegenden Schneidform abnimmt und somit weniger Verfahrweg entsteht. Vorliegend beträgt der Bearbeitungsdurchmesser $35 \mu \mathrm{m}$ (inkl. Wobbeln); sofern ein breiterer Linienabstand gewählt wird, ist kein Spurüberlapp mehr vorhanden. Dies zeigt sich bei einem

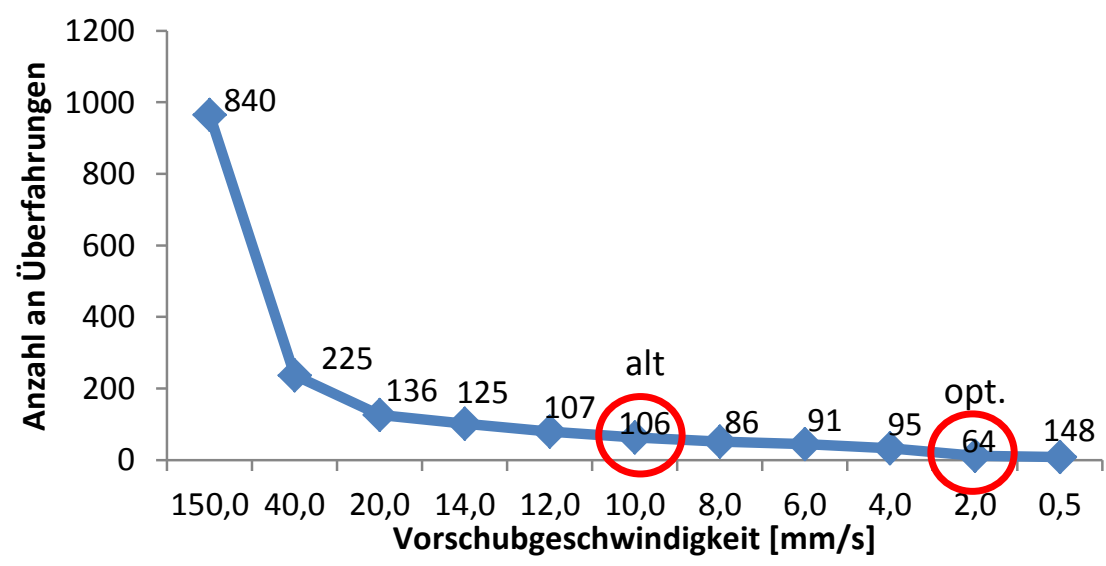

Abb. 46: Einfluss der Vorschubgeschwindigkeit auf den Materialabtrag mit gekennzeichneter Bearbeitungszeit in Sekunden. Messwerte entnommen aus Kagerer, Irlinger und Lueth (2011).

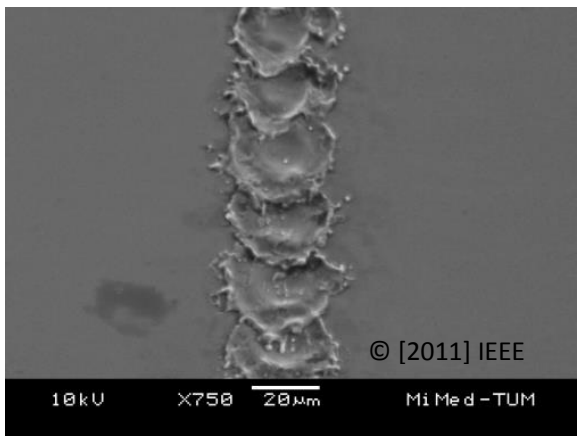

(a)

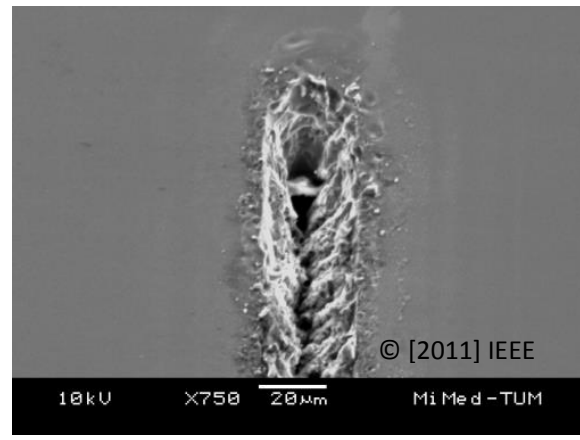

(b)

Abb. 47: Rasterelektronenmikroskopische Aufnahmen zu dem Einfluss der Vorschubgeschwindigkeit auf den Materialabtrag. Einmalige Überfahrung mit dem Laserspot in (a) mit $\mathrm{v}_{\mathrm{s}}=300 \mathrm{~mm} / \mathrm{s}$ und in (b) $\mathrm{v}_{\mathrm{s}}=0,5 \mathrm{~mm} / \mathrm{s}$. Entnommen aus Kagerer, Irlinger und Lueth (2011). 
Linienabstand von $40 \mu \mathrm{m}$ mit einem verbundenen Anstieg der Prozesszeit und der Anzahl an Überfahrungen. Daraus geht hervor, dass mit einer Erhöhung des Linienabstandes auf $30 \mu \mathrm{m}$ (SÜ=14,3 \%) eine Abnahme sowohl von der Anzahl an Überfahrungen von 12 auf 10 als auch von der Bearbeitungszeit von 64 auf 52 s einhergeht. Abb. 48 (b) zeigt, dass trotz Hinzuziehens weiterer Schneidformen und somit einer weiteren Verbreiterung der Schnittfuge, die Anzahl an Überfahrungen nur in geringem Maße reduziert wird. Jedoch steigt gleichzeitig die Prozesszeit. Abb. 49 zeigt Aufnahmen von zwei Laserlinien mit unterschiedlichen SÜs.

\subsubsection{Zusammenfassung der Ergebnisse}

Durch vorliegende Untersuchungen ist es möglich, die Anzahl an notwendigen Überfahrungen von $60 \mathrm{zu}$ Projektbeginn auf 10 nach Projektende zu reduzieren. Gleichzeitig kann die Prozesszeit von 106 auf 52 s reduziert werden. Entscheidend für diese deutliche Reduzierung ist ein optimiertes Zusammenspiel der Laserstrahlquellen unabhängigen Parametern. Der Fokus wird für Laserstrahlschneidaufgaben mittig in das Werkstück gelegt, der PÜ bzw. SÜ beträgt 99,4 \% ( $\left.\mathrm{v}_{\mathrm{s}}=2 \mathrm{~mm} / \mathrm{s}\right)$ bzw. 14,3 \% $(\Delta \mathrm{y}=30 \mu \mathrm{m})$ mit zwei Schneidformen. Dementsprechend kann die Hypothese, mit maximal 10 Überfahrungen einen Ausschnitt in $300 \mu \mathrm{m}$ dickem Silizium zu erzielen, bestätigt werden. Durch die starke Reduzierung der Prozesszeit und der notwendigen Überfahrungen wird an dieser Stelle nochmals deutlich, wie wichtig optimal aufeinander abgestimmte Bearbeitungsparameter für effiziente Laserstrahlschneidaufgaben sind.

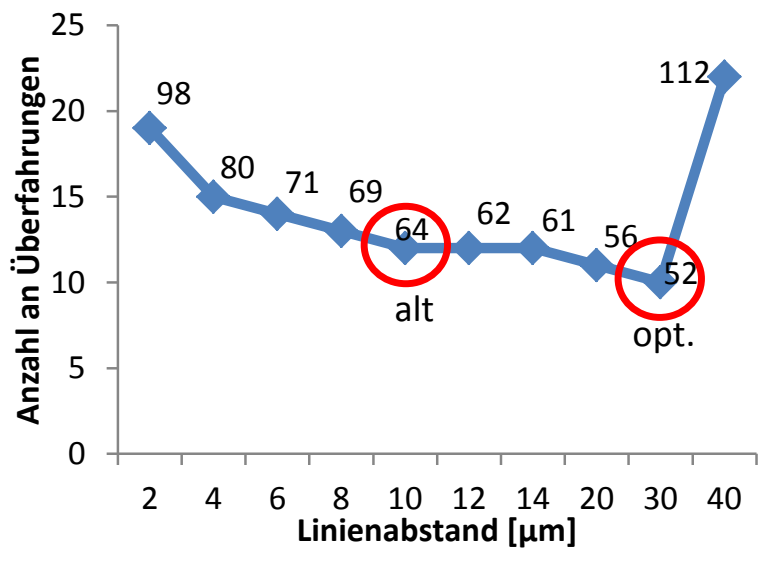

(a)

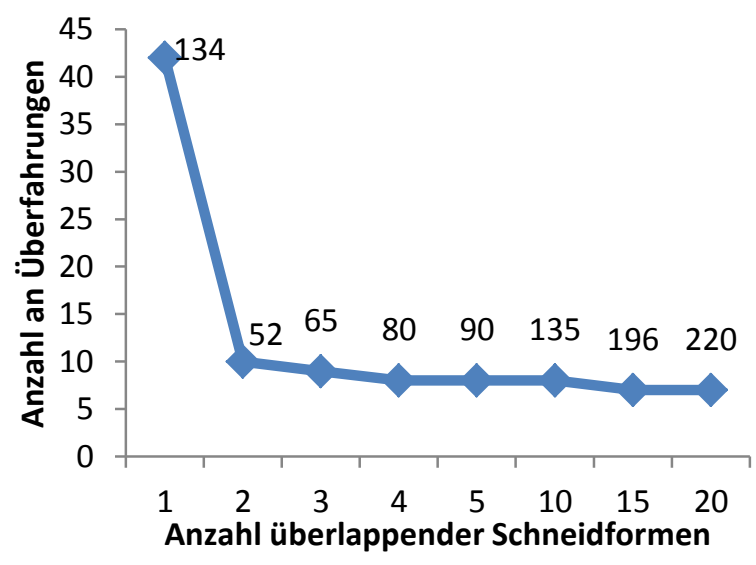

(b)

Abb. 48: (a) Einfluss des Linienabstandes und (b) der Anzahl der überlappenden Schneidformen auf den Materialabtrag mit gekennzeichneter Bearbeitungszeit in Sekunden. Messwerte entnommen aus Kagerer, Irlinger und Lueth (2011).

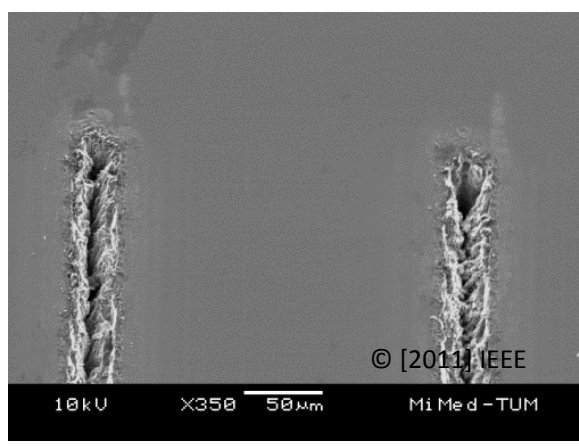

(a)

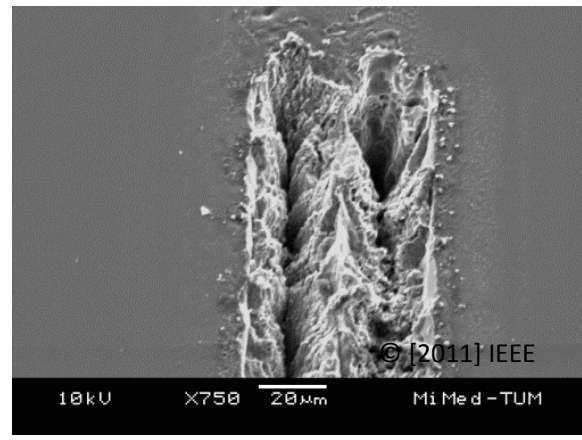

(b)

Abb. 49: Rasterelektronenmikroskopische Aufnahmen von Laserlinien mit (a) keinem Spurüberlapp und (b) mit einem Abstand von $30 \mu \mathrm{m}$. Entnommen aus Kagerer, Irlinger und Lueth (2011). 


\subsection{Experiment 2: Ebener Laserstrahlmaterialabtrag in Silizium}

\subsubsection{Beschreibung des Messverfahrens}

In diesem Experiment gilt es aufzuzeigen, welchen Einfluss die Laserstrahlquellen unabhängigen Parameter auf den ebenen Laserstrahlmaterialabtrag in Silizium, bezogen auf den Bearbeitungsdurchmesser, die Volumenabtragsrate $\left[\mathrm{mm}^{3} / \mathrm{min}\right]$ und auf die Rauheit der abgetragenen Gebiete (Mittenrauhwert $\mathrm{R}_{\mathrm{a}}$ und Rauhtiefe $\mathrm{R}_{\mathrm{z}}$ ), besitzen. Im Detail sind dies die Fokusposition, die Vorschubgeschwindigkeit und der Linienabstand.

Folgende Hypothese soll an dieser Stelle mit dem Experiment bestätigt werden: Es ist möglich, mit einem vergleichsweisen leistungsschwachen IR Lasersystem ( $\mathrm{P}_{\text {Mittel }}=5 \mathrm{~W}$ ) eine Volumenabtragsrate von mindestens $1,5 \mathrm{~mm}^{3} / \mathrm{min}$ mit einer Rauheit $\mathrm{R}_{\mathrm{z}}$ von maximal $15 \mu \mathrm{m}$ bzw. $R_{a}$ von $3 \mu \mathrm{m} \mathrm{zu}$ erreichen, indem die Laserstrahlquellen unabhängigen Parameter optimal aufeinander abgestimmt werden.

Es werden in $525 \mu \mathrm{m}$ dicken Siliziumchips quadratische Taschen mit einer Seitenlänge von $5 \mathrm{~mm}$ bei einer festen Anzahl von vier Überfahrungen abladiert. Die Wahl dieser Form begründet sich dadurch, dass derartige Taschen in Mikrosystemen oft als Fluidreservoire, Pumpkammern oder generell als Vertiefungen für die Integration weiterer Bauteile (wie bspw. Katalysatoren in einer Brennkammer) eingesetzt werden können. Es wird das IR Lasersystem LS 2000 / 8000 (siehe Abb. 28 (b)) mit einer fest eingestellten Pulsfrequenz von 10 kHz und einer mittleren Leistung von $5 \mathrm{~W}$ verwendet. Für die Bestimmung des Bearbeitungsdurchmessers werden die Taschen mit $5 \mathrm{~mm}$ Seitenlänge abladiert. Im Ergebnis ergeben sich Taschen mit größerer Seitenlänge. Die eingestellte Seitenlänge von $5 \mathrm{~mm}$ wird von dieser realen Größe abgezogen, so dass sich der Bearbeitungsdurchmesser $d_{W}$ ergibt. Die Messung der Seitenlänge wird an einem Messtaster der Firma Heidenhain (Modell MT12) mit einer Genauigkeitsklasse von $\pm 0,5 \mu \mathrm{m}$ in Verbindung mit einem Auflichtmikroskop der Firma Carl Zeiss und einem Kreuztisch mit Mikrometerstellschrauben vorgenommen. Für die Messung der Tiefe wird ebenfalls ein Messtaster der Firma Heidenhain (Modell ST3008) mit einer Genauigkeitsklasse von $\pm 1 \mu \mathrm{m}$ verwendet. Hieraus ergibt sich nach (10.3) das abgetragene Volumen $\mathrm{V}_{\mathrm{abt}}$, welches sich nach der Volumenberechnung eines Pyramidenstumpfes aus der Tiefe $\mathrm{T}_{\mathrm{abt}}$ und den jeweiligen Flächen auf der Oberseite $\mathrm{Fl}_{\text {Ober }}$ und am Boden $\mathrm{Fl}_{\text {Boden }}$ richtet.

$$
V_{a b t}=\frac{1}{3} \cdot T_{a b t} \cdot\left(F l_{\text {Ober }}+F l_{\text {Boden }}+\sqrt{F l_{\text {Ober }} \cdot F l_{\text {Boden }}}\right)
$$

Die Rauheitsmessung wird an dem Perthometer S6P der Firma Feinprüf Perthen automatisiert durchgeführt. Es wird sowohl in die Richtung der Laserstrahlbewegung als auch senkrecht hierzu gemessen und hieraus der Mittelwert aus drei Messproben pro Messpunkt gebildet. Die Prozesszeit für vier Überfahrungen wird manuell aufgenommen.

\subsubsection{Experiment zur Messung der Vorteile}

Zu Beginn des Experimentes wird ein Parametersatz gewählt, welcher sukzessive optimiert wird. Hierbei beträgt der Vorschub $30 \mathrm{~mm} / \mathrm{s}$ und der Linienabstand $25 \mu \mathrm{m}$. Es wird eine parallele Linienschraffur gewählt. Als erster Parameter wird die Fokusposition variiert und deren Einfluss auf den Abtrag aufgezeigt. Ist eine optimale Fokusposition gefunden, werden der Vorschub und anschließend der Linienabstand optimiert. Die Abtragsformen werden mit Hil- 
fe der Steuerungssoftware des Galvanometerscanners an dem IR Lasersystem LS 2000 / 8000 erstellt. Das Experiment findet ohne Prozessgas an Luft statt. Vor der Messung erfolgt eine Reinigung der Messproben für zehn Minuten in einem Megaschallbad, um Prozessrückstände bzw. Verunreinigungen zu beseitigen.

Mit dem in Kapitel 9 beschriebenen Fokusfindungsprozess kann auch bei dem IR Lasersystem der Fokus in verschiedenen Schritten durch den $525 \mu \mathrm{m}$ dicken Siliziumchip gelegt werden. In Abb. 50 entspricht eine Fokusposition von $0 \mu \mathrm{m}$ der Werkstückoberfläche. Negative Positionen bedeuten einen Fokus unterhalb der Werkstückoberfläche. Die Untersuchungen zu der Fokusposition zeigen, je weiter der Fokus oberhalb der Oberfläche des Werkstückes liegt, desto größer ist der Bearbeitungsdurchmesser (siehe Abb. 50 (a)). Der Grund hierfür liegt im hyperbolischen Charakter des Gaußschen Strahlprofils. Der Laserstrahl weitet sich auf, so dass ein breiterer Strahl auf der Oberfläche auftrifft. Gleichzeitig nimmt damit auch die Strahlintensität nach (7.5) ab, so dass auch die Ablationsrate abnimmt (siehe Abb. 50 (b)). Darüber hinaus zeigt sich, dass tiefe Fokuslagen im Werkstück (Positionen kleiner als $-250 \mu \mathrm{m})$ zu einer Verringerung des Bearbeitungsdurchmessers führen. Das zu abladierende Gebiet ist bei diesen Positionen zu weit von dem Fokuspunkt entfernt, so dass weniger Material abgetragen wird und sich hierdurch ein schmälerer Schmelzaufwurf um die Eintrittsseite bildet, wodurch letztendlich auch der Bearbeitungsdurchmesser abnimmt. In einem Bereich des Fokus von $\pm 250 \mu \mathrm{m}$ um die Oberfläche des Werkstückes variiert der Bearbeitungsdurchmesser zwischen 80 und $86 \mu \mathrm{m}$. Allerdings führt nur ein Fokus direkt auf der Oberfläche zu der höchsten Volumenabtragsrate von $1,6 \mathrm{~mm}^{3} / \mathrm{min}$. Nach vier Überfahrungen wird eine Ablationstiefe von $135 \mu \mathrm{m}$ erzielt. Die Rauheitsuntersuchungen zeigen, dass die Fokusposition keinen signifikanten Einfluss hierauf besitzt. $R_{\mathrm{z}}$ nimmt dabei Werte zwischen 27 und $35 \mu \mathrm{m}$ und $\mathrm{R}_{\mathrm{a}}$ zwischen 5 und $7 \mu \mathrm{m}$ ein. Es lässt sich an dieser Stelle das Zwischenergebnis ziehen, dass eine Fokusposition auf der Oberseite des Werkstückes zu einer effizienten Bearbeitung mit kleinem Bearbeitungsdurchmesser führt. Je kleiner der Bearbeitungsdurchmesser ist, desto höher ist im Ergebnis die mögliche Strukturauflösung.

Als nächsten Parameter gilt es, die Vorschubgeschwindigkeit zu optimieren. Diese wird in einem Bereich von 5 bis 200 mm/s variiert. Die Ergebnisse zeigen, auch wenn einerseits niedrige Vorschübe respektive hohe Pulsüberlappwerte zu einer höheren Abtragsrate pro Überfahrung führen, wird aufgrund des geringen Vorschubes andererseits jedoch mehr Prozesszeit benötigt. Dies senkt wiederum die wichtige Volumenabtragsrate pro Zeiteinheit. Der effizien-

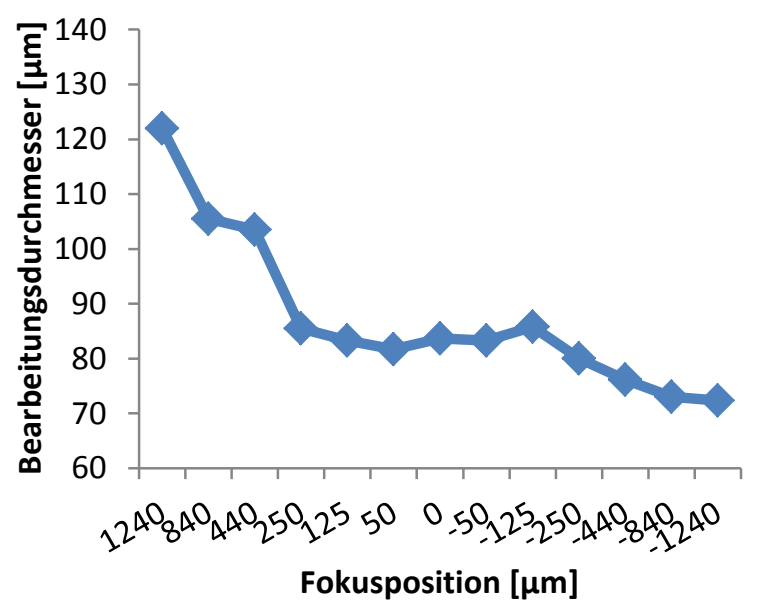

(a)

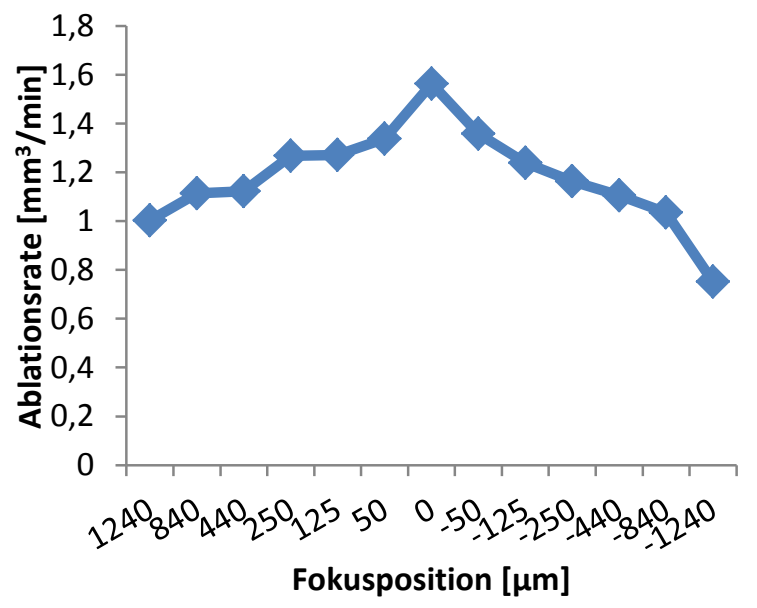

(b)

Abb. 50: Einfluss der Fokusposition auf (a) den Bearbeitungsdurchmesser und in (b) auf die Ablationsrate. Messwerte entnommen aus Kagerer, Irlinger und Lueth (2012). 
teste Wert wird bei einem Vorschub von $100 \mathrm{~mm} / \mathrm{s}$ erreicht (siehe Abb. 51 (a)). Hier beträgt die Abtragsrate $2 \mathrm{~mm}^{3} / \mathrm{min}$ und die erzielte Ablationstiefe beträgt nach vier Überfahrungen $53 \mu \mathrm{m}$. Abb. 51 (b) zeigt, dass Vorschübe kleiner $30 \mathrm{~mm} / \mathrm{s}$ zu einer Vergrößerung des Bearbeitungsdurchmessers führen. Der Grund ist die höhere Abtragsrate pro Überfahrung, welche zu einem breiteren Schmelzaufwurf an der Eintrittsseite des Laserstrahls führt. Zudem geht aus Abb. 51 (c) hervor, dass hohe Vorschübe und die damit verbundenen kleinen Pulsüberlappwerte zu einer Reduzierung der Rauheit führen. Der Grund hierfür ist, dass das verdampfte Material mehr Platz zum Entweichen hat, ohne dabei vom nächsten Puls getroffen zu werden und so dessen Licht durch Streuung gestört wird. Die Rauhtiefe $R_{z}$ wird von $30 \mu \mathrm{m}$ $\left(\mathrm{v}_{\mathrm{s}}=30 \mathrm{~mm} / \mathrm{s}\right)$ auf $15 \mu \mathrm{m}\left(\mathrm{v}_{\mathrm{s}}=100 \mathrm{~mm} / \mathrm{s}\right)$ reduziert. Als Resümee kann daher an dieser Stelle festgehalten werden, auch wenn niedrige Vorschübe zu einer höheren Abtragsrate pro Überfahrung führen, wird aufgrund des geringen Vorschubes mehr Prozesszeit benötigt und die Volumenabtragsrate pro Zeiteinheit sinkt. Zudem vergrößern sich die Rauheit und der Bearbeitungsdurchmesser.

Den letzten Laserstrahlquellen unabhängigen Parameter, den es zu optimieren gilt, stellt der Linienabstand dar, welcher mit dem Bearbeitungsdurchmesser den Spurüberlapp bestimmt. Bislang besitzen die parallel laufenden Schraffurlinien einen Abstand von $25 \mu \mathrm{m}$. Die Ergebnisse aus Abb. 52 (a) zeigen, dass der gewählte Linienabstand von $25 \mu \mathrm{m}$ gut gewählt ist und zu der höchsten Volumenabtragsrate führt. Geringere Linienabstände reduzieren die Volumenabtragsrate, da in der Summe mehr Linien für die Schraffur und somit mehr Prozesszeit benötigt werden. Größere Linienabstände respektive kleinere Spurüberlappwerte rufen eben-

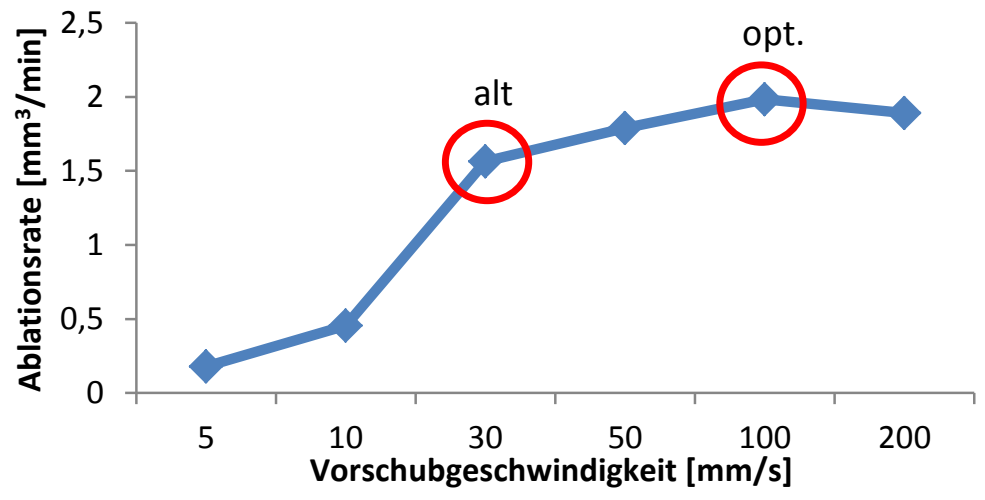

(a)

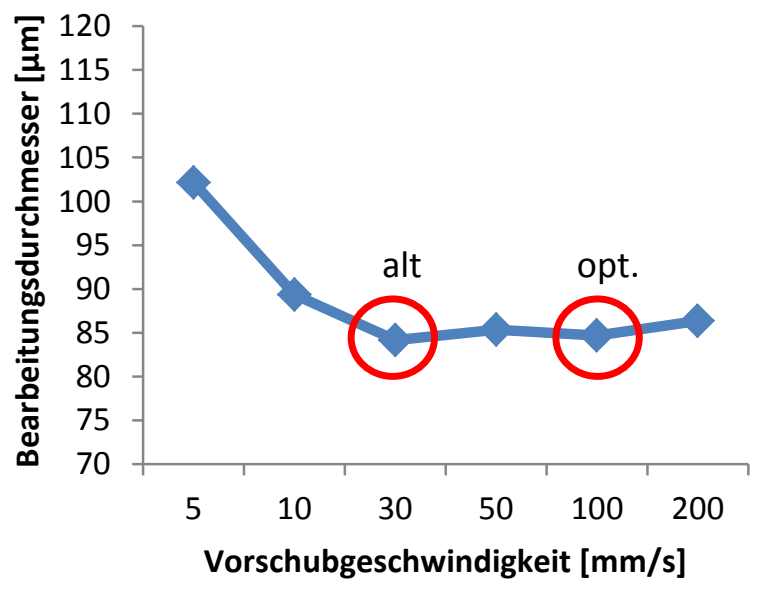

(b)

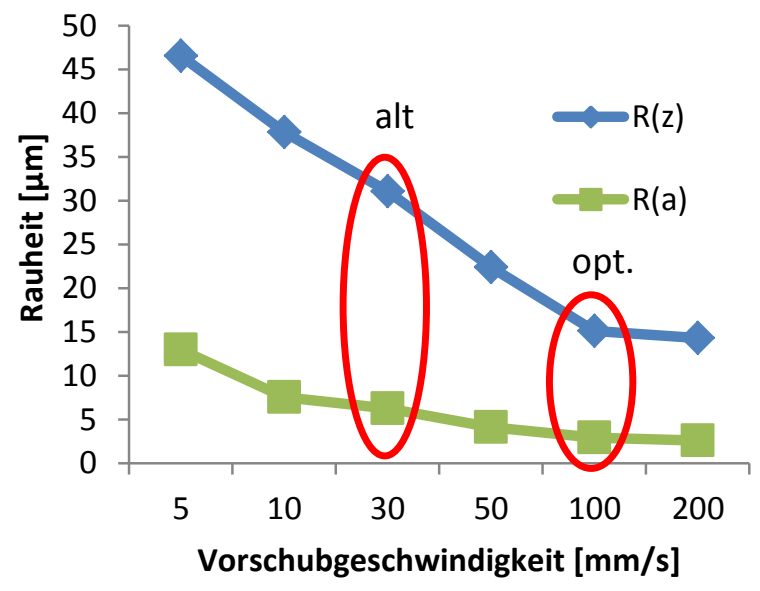

(c)

Abb. 51: Einfluss des Vorschubes auf (a) die Ablationsrate, (b) den Bearbeitungsdurchmesser und (c) die Rauheit. Messwerte entnommen aus Kagerer, Irlinger und Lueth (2012). 
falls eine Reduzierung der Volumenabtragsrate hervor, da weniger Energie des Laserstrahls auf die gleiche Stelle trifft. Abb. 52 (b) zeigt den Einfluss des Linienabstandes auf die Rauheit der abgetragenen Fläche. Auch hier ist erkennbar, dass bei Erhöhung des Linienabstandes ebenfalls die Rauheitswerte zunehmen. Durch den größer werdenden Abstand nimmt der Boden des abladierten Gebietes eine wellige Form an (siehe Abb. 53). Die Messung des Bearbeitungsdurchmessers zeigt, dass der Linienabstand keinen signifikanten Einfluss hierauf besitzt. Es kann daher das Zwischenfazit gezogen werden, dass eine hohe Ablationsrate bei gleichzeitig geringer Rauheit bei einem Linienabstand von $25 \mu \mathrm{m}$ erzielt wird.

\subsubsection{Zusammenfassung der Ergebnisse}

Das Zusammenspiel der Laserstrahlquellen unabhängigen Parameter auf den ebenen Laserstrahlmaterialabtrag ist aufgezeigt und optimiert. Um dabei eine hohe Volumenabtragsrate, einen kleinen Bearbeitungsdurchmesser und eine geringe Rauheit zu erhalten gilt: Der Fokus liegt auf der Oberfläche des Werkstuickes und der Spur- bzw. Pulsüberlapp beträgt 70,2 \% $(\Delta \mathrm{y}=25 \mu \mathrm{m})$ bzw. 88,2 \% $\left(\mathrm{v}_{\mathrm{s}}=100 \mathrm{~mm} / \mathrm{s}\right)$. Der Bearbeitungsdurchmesser in Silizium liegt bei $84 \mu \mathrm{m}$ und kann bei der Dimensionierung der Abtragsgeometrien für die Erstellung von Funktionsmodellen berücksichtigt werden. Die Ablationstiefe beträgt nach vier Überfahrungen $53 \mu \mathrm{m}$. Des Weiteren liegt durch die umfangreichen Experimente eine Wertetabelle der Bearbeitungsparameter für unterschiedliche Ablationstiefen vor. Die Hypothese, eine Volumenabtragsrate von mindestens $1,5 \mathrm{~mm}^{3} / \mathrm{min}$ bei einer maximalen Rauheit $\mathrm{R}_{\mathrm{z}}$ von $15 \mu \mathrm{m}$ bzw. $R_{a}$ von $3 \mu \mathrm{m}$ zu erzielen, ist bestätigt.

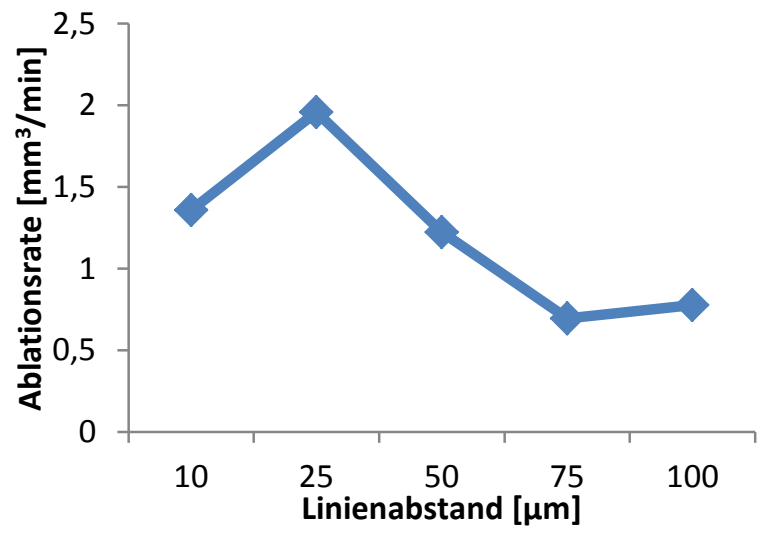

(a)

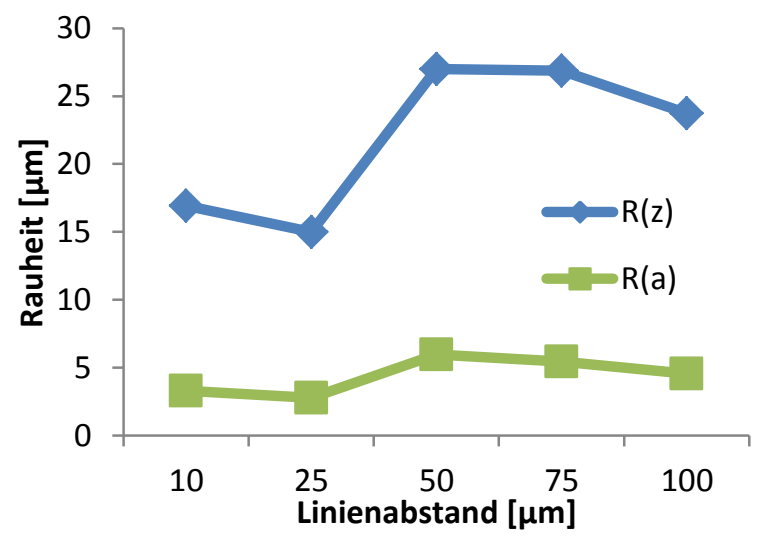

(b)

Abb. 52: Einfluss des Linienabstandes auf (a) die Ablationsrate und in (b) auf die Rauheit. Messwerte entnommen aus Kagerer, Irlinger und Lueth (2012).

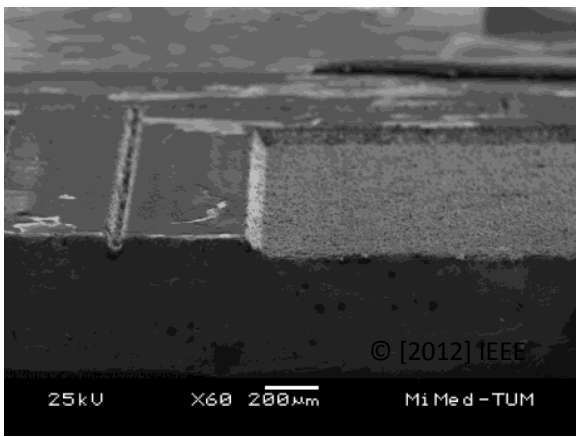

(a)

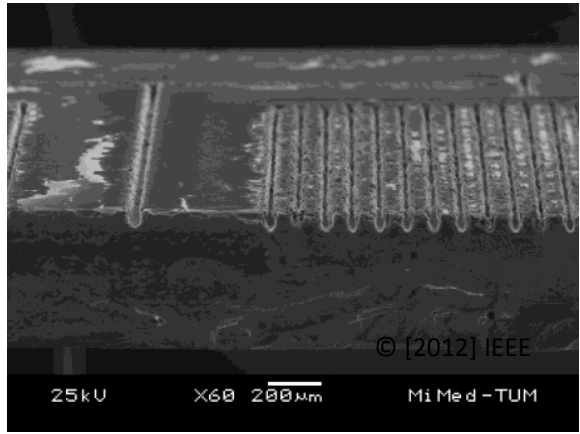

(b)

Abb. 53: Rasterelektronenmikroskopische Aufnahmen zu dem Einfluss des Linienabstandes auf den Materialabtrag. Viermalige Überfahrung in (a) mit einem Linienabstand von $\Delta y=25 \mu \mathrm{m}$ und in (b) mit keinem Überlapp. Entnommen aus Kagerer, Irlinger und Lueth (2012). 


\subsection{Experiment 3: Fertigung senkrechter Kanäle mithilfe des Trennschleifens}

\subsubsection{Beschreibung des Messverfahrens}

In diesem Experiment gilt es aufzuzeigen, ob der in Kapitel 7 vorgestellte Trennschleifprozess es ermöglicht, unterschiedlich breite und tiefe Kanäle in Siliziumsubstraten zu erstellen.

Folgende Hypothese soll an dieser Stelle mit dem Experiment bestätigt werden: Es ist mit dem vorgestellten Trennschleifprozess möglich, sowohl unterschiedlich breite als auch tiefe Kanäle mit einem mittleren absoluten Fehler von maximal $5 \mu \mathrm{m}$ zu erstellen. Die minimale Kanalbreite beträgt dabei $50 \mu \mathrm{m}$.

Es werden in $525 \mu \mathrm{m}$ dicken Siliziumchips die unterschiedlich dimensionierten Kanäle mit $2 \mathrm{~mm}$ Länge als direkte Fortführung einer $100 \mu \mathrm{m}$ tief abladierten Tasche erstellt. Die Siliziumchips werden auf die UV empfindliche Sägefolie 1027R der Firma Ultron Systems geklebt. Es wird das Siliziumsägeblatt FTB R46 45 x 100 von der Firma Mitsubishi Corporation gewählt. Dieses besitzt eine Körnung zwischen 4 und $6 \mu \mathrm{m}$, eine Blattdicke von $45 \mu \mathrm{m}$ und einen Blattüberstand von $1,1 \mathrm{~mm}$. Die Bearbeitungsparameter der Wafersäge DISCO DAD321 sind ein Vorschub von $5 \mathrm{~mm} / \mathrm{s}$ und eine Drehzahl von $38000 \mathrm{U} / \mathrm{min}$. Es werden drei Kanäle quadratischen Querschnittes (Breite=Tiefe) von 50, 70 und $100 \mu \mathrm{m}$ erstellt. Der einzustellende Versatz wird zu höchstens einem Drittel der Sägeblattbreite gewählt, um eine Rillenbildung auf dem Boden des Kanals zu vermeiden. Der Versatz wird nach (7.14) berechnet. Für einen $50 \mu \mathrm{m}$ breiten Kanal reicht lediglich ein Schnitt aus, da sich dieser durch Kerf und Chipping gegenüber der nominalen Blattdicke verbreitert. Für die 70 bzw. $100 \mu \mathrm{m}$ breiten Kanäle werden insgesamt drei bzw. fünf Schnitte mit einem Versatz von je 12,5 bzw. 13,5 $\mu \mathrm{m}$ eingesetzt. Es wird zu Beginn ein zentraler Schnitt ausgeführt; im Anschluss alternieren die Schnitte um den jeweiligen Versatz um den zentralen Schnitt seitlich. Die Messung der Breite der Kanäle wird direkt mit der integrierten Messfunktion der Wafersäge durchgeführt, welche eine absolute Genauigkeit von $5 \mu \mathrm{m}$ bei $160 \mathrm{~mm}$ Verfahrweg aufweist. Für die Messung der Tiefe wird ein Messtaster der Firma Heidenhain (Modell MT12) mit einer Genauigkeitsklasse von $\pm 0,5 \mu \mathrm{m}$ in Verbindung mit einem Auflichtmikroskop der Firma Carl Zeiss verwendet. Das Messprinzip basiert auf der Fokusverschiebung zwischen dem Kanalboden und der Substratoberseite. Es wird der Mittelwert aus mindestens 48 Messproben pro Messpunkt gebildet.

\subsubsection{Experiment zur Messung der Vorteile}

Vor dem ersten Schnitt wird das Sägeblatt an einer Schärfplatte gedresst, um überstehende Schleifkörner zu entfernen. Im Anschluss erfolgt automatisiert eine Bestimmung des verbleibenden Blattüberstandes. Nach der Durchführung dieser Routine ist die Maschine einsatzbereit. Die Schnitte werden ausgeführt. Für die Bestimmung der Nulllage wird die Sägespindel in Schritten von maximal $5 \mu \mathrm{m}$ in Richtung des Werkstückes verfahren und ein Probeschnitt ausgeführt. Der Schnitt erfolgt an einer Stelle auf dem Werkstück, an der dieser für die weiteren Prozesse nicht störend wirkt, die aber dennoch in unmittelbarer Nähe zu der geforderten Sollschnittposition ist. Diese Schrittfolge wird solange wiederholt, bis eine Kerbe auf der Oberfläche sichtbar ist. Diese Höhenposition stellt die Nulllage für den Schnitt dar. Im Anschluss wird die Sägespindel an die gewünschte Position und in die geforderte Kanaltiefe verfahren. Nach der Durchführung der Schnitte werden die Siliziumchips zur Lösung von der Sägefolie in ein UV Belichtungsgerät gegeben und gelöst. Vor der Messung erfolgt eine Reinigung der Messproben für zehn Minuten in einem Megaschallbad, um Prozessrückstände bzw. Verunreinigungen zu beseitigen. 
Abb. 54 zeigt die Ergebnisse für das Trennschleifen. Sowohl die Kanalbreite als auch die Kanaltiefe werden mit der von Kapitel 4 geforderten Genauigkeit von $\pm 5 \mu \mathrm{m}$ gefertigt. Die resultierenden Standardabweichungen sind bei der Messung der Kanaltiefen geringfügig größer, was auf das eingesetzte Messprinzip, beruhend auf der Fokusverschiebung, zurückzuführen ist. Die Kanäle weisen senkrechte Seitenwände auf und es sind keine Bodenrillen aufgrund der überlappenden Schnitte sichtbar (siehe Abb. 55). Einzig zu erkennen ist ein Fußrundungsradius an den Seitenwänden im Übergang zu dem Boden, welcher prozessspezifisch durch das Trennschleifen mit runden Sägeblättern entsteht.

\subsubsection{Zusammenfassung der Ergebnisse}

Durch vorliegende Untersuchungen ist es möglich, Kanäle in Siliziumsubstraten mit unterschiedlicher Breite und Tiefe zu fertigen. Durch den Einsatz überlappender Schnitte ist die Kanalbreite unabhängig von der Sägeblattbreite. Die Bestimmung der Nulllage für den ersten Schnitt ermöglicht es, reproduzierbar gefertigte Kanaltiefen zu erzielen. Die erstellten Kanäle werden in mikrofluidischen Systemen allgemein als Fluidkanäle und insbesondere bei Tropfenerzeugern als Düsen eingesetzt. Die Kanäle besitzen keine sichtbare Rauheit (Minimierung von Reibung in der Mikrofluidik) an den Seitenwänden, die senkrecht verlaufen. Es sind keine Rillen am Boden zu erkennen. Es ist vielmehr eine definierte Kanalform erstellbar. Somit wird die Hypothese, Kanäle mit einem mittleren absoluten Fehler von maximal $5 \mu \mathrm{m}$ bzgl. ihrer Breite und Tiefe in Siliziumsubstraten zu fertigen, bestätigt.

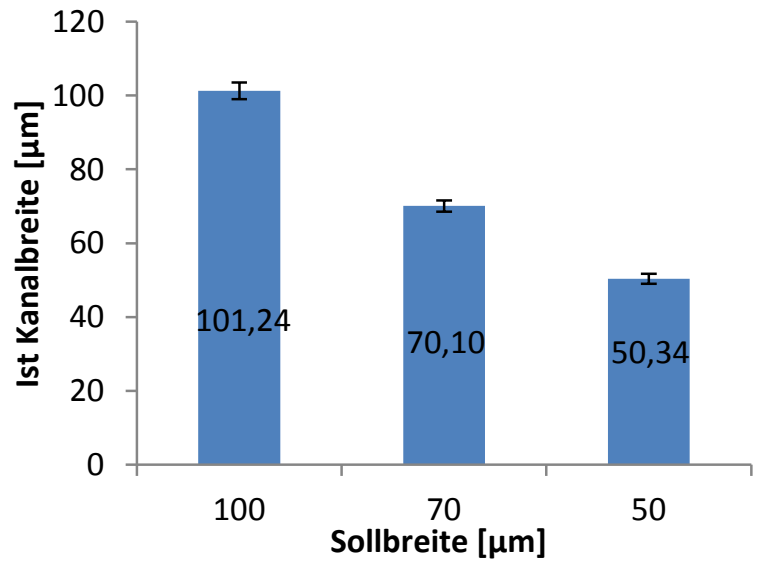

(a)

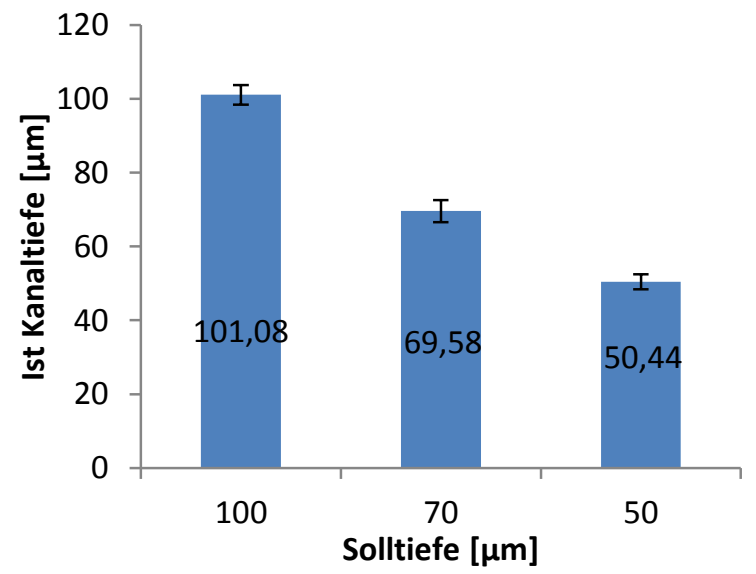

(b)

Abb. 54: Messergebnisse zu der Erstellung von Kanälen in Silizium unter Variation in (a) ihrer Breite und in (b) ihrer Tiefe.

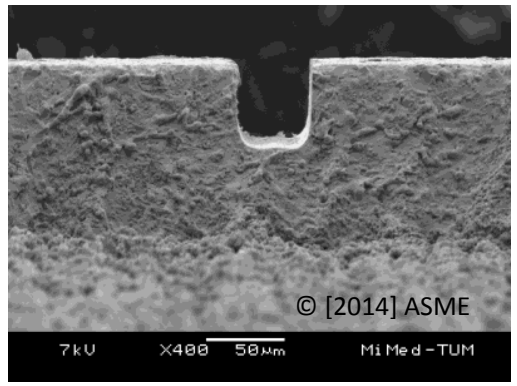

(a)

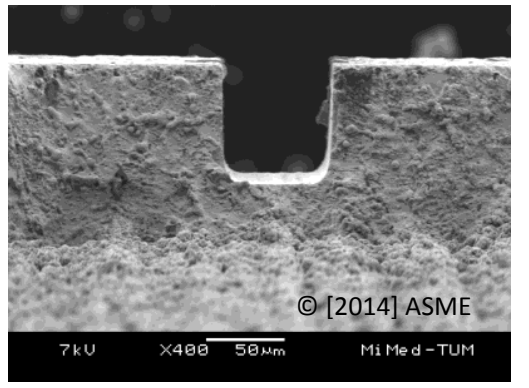

(b)

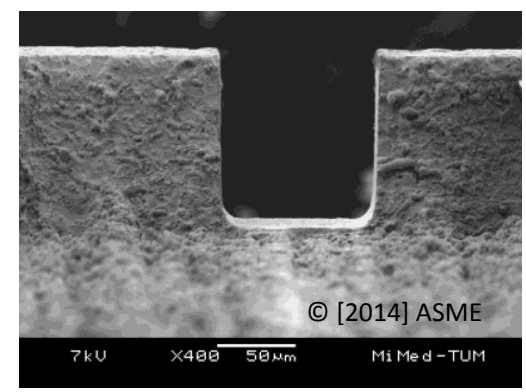

(c)

Abb. 55: Rasterelektronenmikroskopische Aufnahmen von Kanälen, hergestellt durch das Trennschleifen. Die Breite entspricht der Tiefe in (a) $50 \mu \mathrm{m}$, (b) $70 \mu \mathrm{m}$ und (c) $100 \mu \mathrm{m}$. Entnommen aus Kagerer et al. (2014). 


\subsection{Experiment 4: Positionierung zweier Bauteile mit dem Mikromontageplatz}

\subsubsection{Beschreibung des Messverfahrens}

In diesem Experiment gilt es aufzuzeigen, mit welcher Genauigkeit Relativpositionierungsaufgaben mit dem Mikromontageplatz möglich sind. Es werden zwei planare Bauteile / Substrate in einem fest definierten Kantenabstand miteinander gefügt.

Folgende Hypothese soll an dieser Stelle mit dem Experiment bestätigt werden: Es ist möglich, mit dem erstellten Mikromontageplatz Relativpositionierungsaufgaben sowohl mit Mikrometer- als auch mit Millimeterabständen von zwei planaren Bauteilen / Substraten mit einem mittleren absoluten Fehler von maximal $20 \mu \mathrm{m}$ durchzuführen.

Als planare Bauteile werden zwei $525 \mu \mathrm{m}$ dicke Siliziumchips verwendet. Das Substrat auf der Montageplattform besitzt die Dimensionen von $20 \times 25 \mathrm{~mm}^{2}$; das abzusetzende Substrat auf dem Vakuumsauggreifer besitzt die Abmessungen von $15 \times 25 \mathrm{~mm}^{2}$. Die Partner werden mit einem Sekundenkleber miteinander verbunden. Es werden fünf Abstände in vorliegendem Experiment untersucht: 200, 400, 800, 1600 und $3200 \mu \mathrm{m}$. Somit werden Dimensionen sowohl aus der Mikro- als auch aus der Makrowelt verwendet. Die Untersuchung von größeren Abständen ist nicht notwendig, da Relativpositionierungsaufgaben meist in geringem Abstand von einzelnen Strukturen auf dem Zielbauteil auszuführen sind. Gleichwohl bietet vorliegender Mikromontageplatz die Möglichkeit, Abstände von bis zu 50 mm (Verfahrweg Kreuztisch) zu erzielen. Die Messung der Abstände beider Bauteile zueinander wird mit der integrierten Messfunktion der Wafersäge DISCO DAD321 durchgeführt, welche eine absolute Genauigkeit von $5 \mu \mathrm{m}$ bei $160 \mathrm{~mm}$ Verfahrweg aufweist. Hierbei wird sowohl an der oberen als auch an der unteren Ecke des abzusetzenden Bauteiles der Abstand zu der Kante des Substrates, von der aus der Abstand eingestellt worden ist, gemessen. Neben der Abstandsmessung beider Bauteile wird auch deren Parallelität mit Hilfe des auftretenden Winkelversatzes $\gamma$ zueinander betrachtet. Hierzu zeigt (10.4) die Berechnungsvorschrift. Die Abstandsmaße an der oberen bzw. unteren Ecke stellen $a_{\text {oben }}$ bzw. $a_{\text {unten }}$ dar. Die Breite $b_{\text {Sub }}$ der Substrate beträgt $25 \mathrm{~mm}$. Es wird der Mittelwert aus mindestens vier Messproben pro Messpunkt gebildet. Der eingesetzte Sauggreifer wird von der Firma Festo bezogen, welcher einen Durchmesser von $10 \mathrm{~mm}$ besitzt, eine runde bzw. flache Ausführung aufweist und aus Nitrilkautschuk gefertigt ist (Modell ESS $10 \mathrm{SN}$ ).

$$
\gamma=\arcsin \left(\frac{\left|a_{\text {oben }}-a_{\text {unten }}\right|}{b_{\text {Sub }}}\right)
$$

\subsubsection{Experiment zur Messung der Vorteile}

Vor dem Experiment werden die beiden Fügepartner für 10 Minuten in einem Megaschallbad gereinigt, um eventuelle Verunreinigungen auf den Substraten für den Fügeprozess zu beseitigen. Im Anschluss wird das abzusetzende Bauteil mit dem Sauggreifer aufgenommen und das Substrat auf der Montageplattform fixiert. Hier erfolgt erstmals eine grobe Ausrichtung beider Bauteile zueinander. Sowohl mit der Höhenachse, an der die Montageplattform angebracht ist, als auch mit dem Greif- / Absetzwerkzeug werden beide Bauteile zueinander angenähert, bis ein Abstand von ein bis zwei Millimetern besteht. Es werden beide Videomikroskope mit Hilfe ihrer Haltearme so positioniert, dass die Kanten beider Bauteile, von denen aus die Relativpositionierung ausgeführt wird, und die dazugehörigen Ecken der Bau- 
teile sichtbar sind. Mit Hilfe der Mikrometerstellschrauben an dem X- / Y-Kreuztisch und auch mit Hilfe des Drehtellers werden beide Bauteile zueinander parallel und mit überdeckenden Ecken ausgerichtet (siehe Abb. 56 (a) bis (c)). Im Anschluss wird der Messtaster an die zu verfahrene Achse seitlich angebracht. Das abzusetzende Bauteil wird angehoben, der gewünschte Abstand wird mit Hilfe der Mikrometerstellschrauben verfahren (siehe Abb. 56 (d)), der Sekundenkleber wird auf das Substrat aufgetragen und das abzusetzende Bauteil wird durch eine Abwärtsbewegung des Sauggreifers abgesetzt. Nach einer Dauer von ca. einer Minute wird die Vakuumansaugung beider Bauteile gelöst und der Abstand gemessen. Abb. 57 zeigt Aufnahmen der beiden Videomikroskope bei einer Positionieraufgabe.

Abb. 58 (a) gibt die erzielten Ergebnisse für Relativpositionierungsaufgaben mit dem Mikromontageplatz wieder. Es werden die geforderten Soll- den tatsächlichen Istabständen gegenübergestellt und miteinander verglichen. Das Resultat ist, dass für alle fünf untersuchten Abstände die geforderte Positioniergenauigkeit von $\pm 20 \mu \mathrm{m}$ erreicht wird. Abb. 58 (b) zeigt die erzielten Ergebnisse für die Parallelität der beiden Bauteile zueinander auf. Hierfür wird der mittlere Winkelversatz nach (10.4) berechnet. Für alle fünf untersuchten Abstände ist der Winkelversatz, bezogen auf eine Breite des Werkstiuckes von $25 \mathrm{~mm}$, unter 0,04 ${ }^{\circ}$. Die vergleichsweisen größten Werte treten bei den größten untersuchten Abständen von 1600 und $3200 \mu \mathrm{m}$ auf.

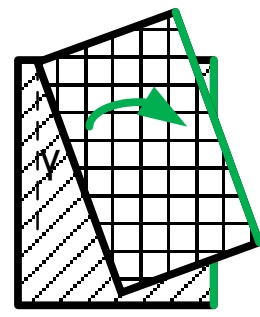

(a)

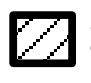
Zielbauteil

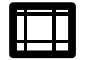
Abzusetzendes Bauteil

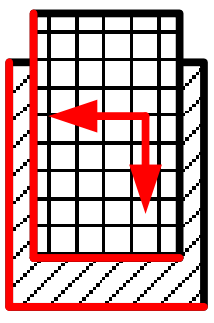

(b)

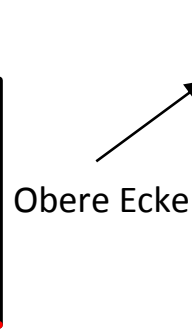

(c)

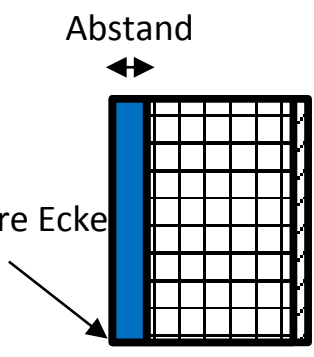

(d)

Abb. 56: Schematische Darstellung des Experimentes der Relativpositionierung. (a) Ausgleich des Winkelversatzes, (b) und (c) Ecke-Ecke Positionierung und (d) Einstellung Abstand KanteKante der Bauteile zueinander.

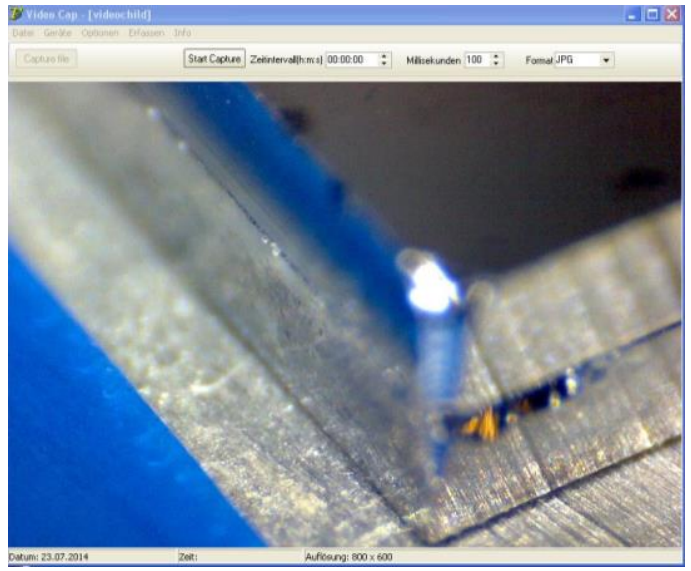

(a)

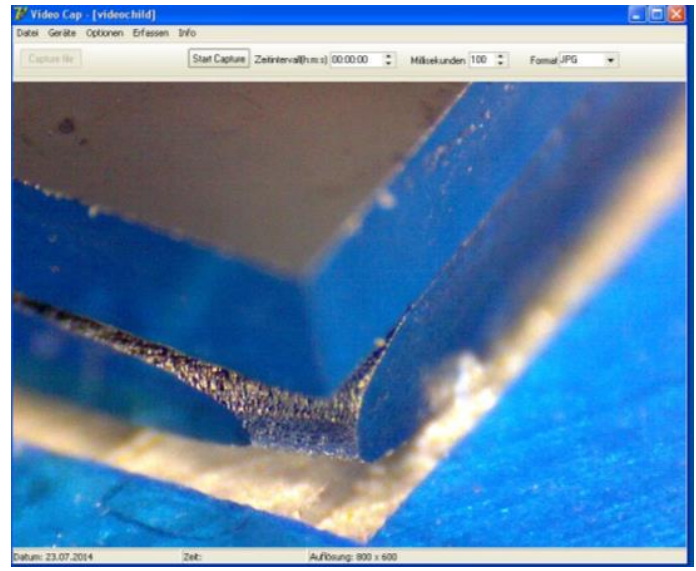

(b)

Abb. 57: Aufnahmen mit den angebrachten Videomikroskopen an dem Mikromontageplatz. Erfolgreiche Ausrichtung beider Bauteile hinsichtlich Parallelität und Eckenüberschneidung in (a) für die untere und in (b) für die obere Ecke. 


\subsubsection{Zusammenfassung der Ergebnisse}

Durch vorliegende Untersuchungen wird gezeigt, dass mit dem entworfenen Mikromontageplatz Relativpositionierungsaufgaben von zwei planaren Bauteilen mit der geforderten Genauigkeit aus Kapitel 4 möglich sind. Die Parallelität der beiden Bauteile ist gewährleistet. Die eingesetzten Videomikroskope bieten eine qualitativ gute optische Wiedergabe des Positioniervorganges, welcher zusätzlich noch durch die Verwendung des Mikrotasters erleichtert wird. Der Einsatz von Haltearmen für die Befestigung der Videomikroskope und des Messtasters bietet eine hohe Flexibilität bzgl. der Integration dieser Hilfsmittel in den Prozess. Abb. 59 zeigt rasterelektronenmikroskopische Aufnahmen der relativ positionierten Bauteile. Die Hypothese, Relativpositionierungen zweier Bauteile mit einem mittleren absoluten Fehler von maximal $20 \mu \mathrm{m}$ zu erzielen, kann mit vorliegendem Experiment bestätigt werden.

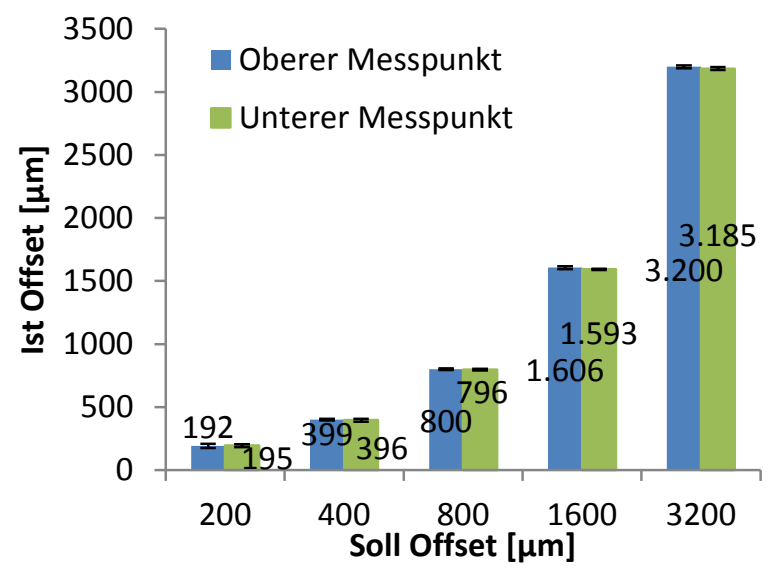

(a)

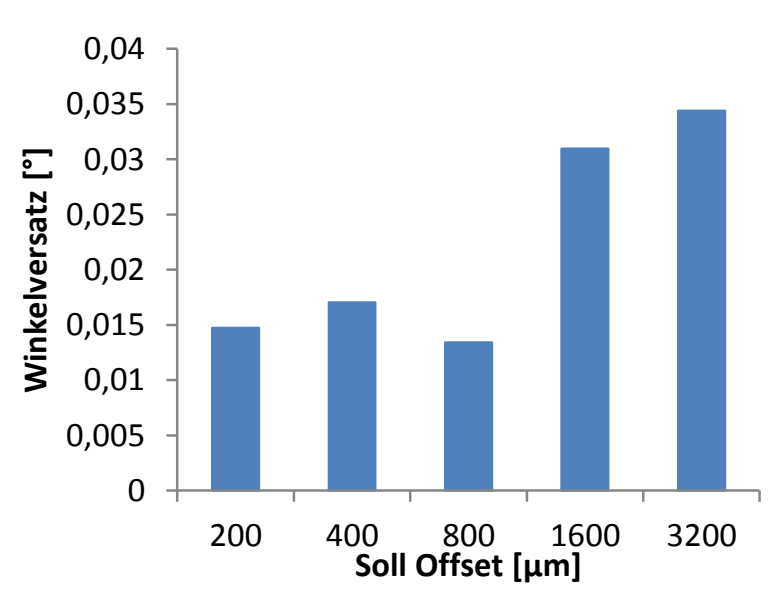

(b)

Abb. 58: Messergebnisse zu der Relativpositionierung. (a) Gegenüberstellung von Soll zu Ist Offset und (b) resultierender Winkelversatz.

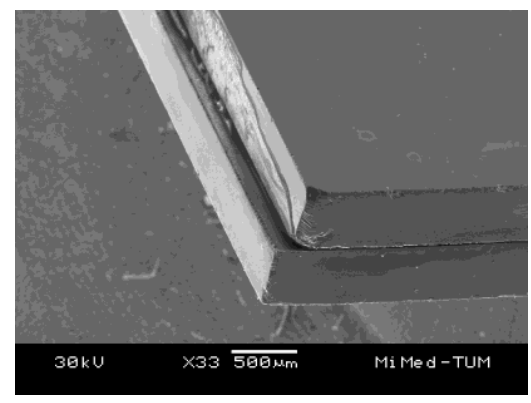

(a)

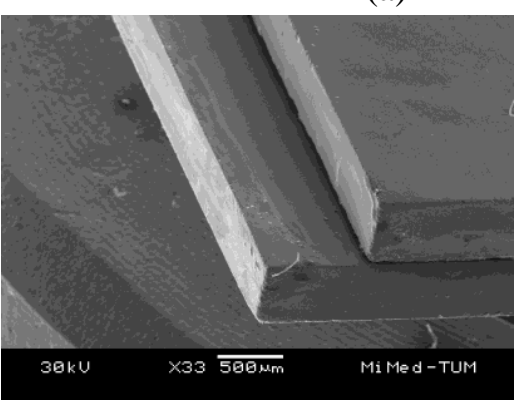

(c)

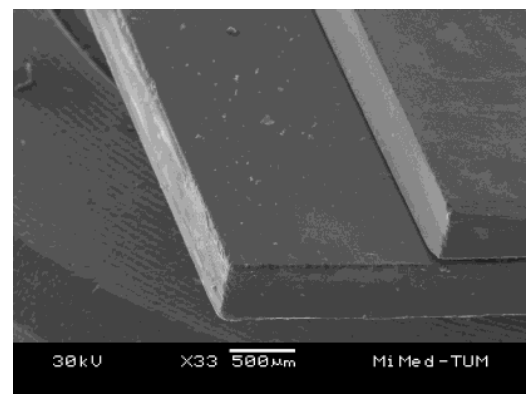

(d)

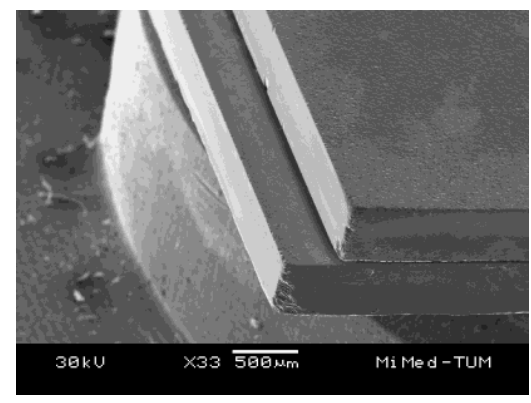

(b)

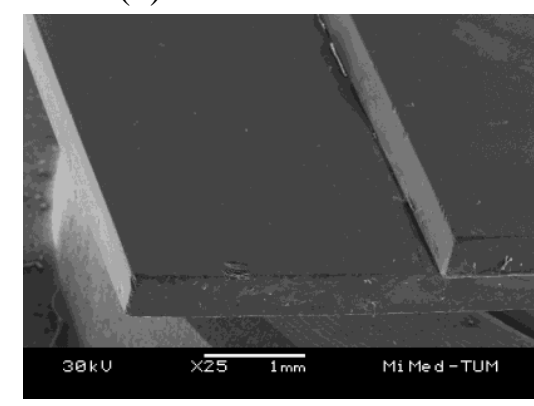

(e)

Abb. 59: Rasterelektronenmikroskopische Aufnahmen für die Relativpositionierung der Siliziumchips mit einem Abstand von (a) $200 \mu \mathrm{m}$, (b) $400 \mu \mathrm{m}$, (c) $800 \mu \mathrm{m}$, (d) $1600 \mu \mathrm{m}$ und (e) $3200 \mu \mathrm{m}$. 


\subsection{Experiment 5: Klebstoffauftrag mit dem Mikromontageplatz}

\subsubsection{Beschreibung des Messverfahrens}

In diesem Experiment gilt es aufzuzeigen, ob mit dem an den Mikromontageplatz angebrachten Klebstoffauftragswerkzeug homogene, ganzflächige und in den Dicken gezielt einstellbare Klebschichten auf Substraten aufgebracht werden können.

Folgende Hypothese soll an dieser Stelle mit dem Experiment bestätigt werden: Es ist mit dem Werkzeug möglich, Klebschichten über eine Fläche von mindestens $10 \mathrm{~cm}^{2}$ mit einem mittleren absoluten Fehler von maximal $3 \mu \mathrm{m}$ (angestrebte Dicke: 10 bis einschl. $20 \mu \mathrm{m}$ ) bzw. $6 \mu \mathrm{m}$ (angestrebte Dicke: größer 20 bis einschl. $40 \mu \mathrm{m}$ ) gegenüber der eingestellten Höhe an der Linearachse der Rakeleinheit auf Substraten aufzutragen.

Die Klebschichten werden auf $500 \mu \mathrm{m}$ dicken geviertelten 4 Zoll Borosilikatglaswafern (Fläche ca. $20 \mathrm{~cm}^{2}$ ) aufgebracht. Die Wahl auf Borosilikatglas begründet sich dadurch, dass dieses oft in der Mikrosystemtechnik als Membran für die Verklebung mit piezoelektrischen Wandlern und auch als anodischer Bondpartner mit Silizium eingesetzt wird. Es werden zwei Klebstoffe verwendet. Zum einen ist dies der Einkomponenten Klebstoff DELOMONOPOX ${ }^{\circledR} 6093$ der Firma DELO Industrie Klebstoffe und zum anderen der Zweikomponenten Klebstoff UHU PLUS ENDFEST 300 der Firma UHU.

Es werden dabei Klebschichten mit einer angestrebten Dicke von 10, 20, 30 und $40 \mu \mathrm{m}$ für beide Klebstoffe auf den Wafervierteln aufgebracht. Für den Zweikomponenten Klebstoff werden zudem auch noch Klebschichtdicken von 50, 70 und $100 \mu \mathrm{m}$ untersucht. Der Zweikomponenten Klebstoff wird in einem Verhältnis von 1:1 (Härter zu Binder) gemischt. Um die Aushärtedauer beider Klebstoffe zu verkürzen und gleichzeitig auch ihre Festigkeit zu erhöhen (UHU, 2015), erfolgt die Aushärtung unter einen erhöhten Temperatureinfluss bei $70{ }^{\circ} \mathrm{C} / 45 \mathrm{~min}(\mathrm{UHU})$ und $110^{\circ} \mathrm{C} / 90 \mathrm{~min}$ (DELO) auf einer externen Heizplatte.

Die geviertelten 4 Zoll Wafer werden für das Experiment mit 17 Messpunkten versehen (siehe Abb. 60). An diesen Messpunkten wird sowohl vor als auch nach dem Klebstoffauftrag die Dicke des Werkstückes gemessen. Die Differenz ergibt die erzielte Klebschichtdicke. Es wird für die Messung ein Messtaster der Firma Heidenhain (Modell ST3008) mit einer Genauigkeitsklasse von $\pm 1 \mu \mathrm{m}$ verwendet. Nach Pfeifer (1982) gilt, dass die Dicke der Klebschicht so gering wie möglich gehalten werden muss, um auch die Biegesteifigkeit des Membranaktors gering zu halten. Daher werden für einen wichtigen Bereich von Klebschichtdicken zwischen 10 und $40 \mu \mathrm{m}$ vier Substrate verwendet. Die restlichen Klebschichtdicken werden an drei Substraten validiert. Es wird jeweils der Mittelwert über alle Messpunkte pro angestrebter Klebschichtdicke gebildet.

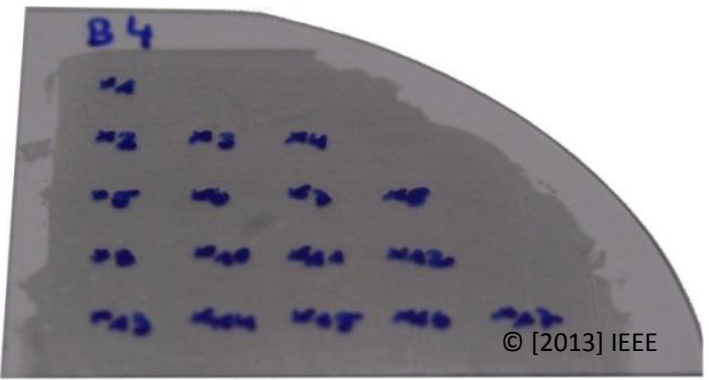

Abb. 60: Substrat für den Klebstoffauftrag aus $500 \mu \mathrm{m}$ dickem Borosilikatglas mit 17 Messpunkten. Entnommen aus Kagerer et al. (2013b). 


\subsubsection{Experiment zur Messung der Vorteile}

Vor dem Experiment werden die Borosilikatglaswafer mit einer Läppfolie mit $30 \mu \mathrm{m}$ Körnung kurz angeschliffen. DIN EN 13887 (Stand November 2003) empfiehlt, vor dem Klebeprozess die Oberfläche mit Läppfolie aufzurauen, um die Leistungsfähigkeit der Klebeverbindung zu erhöhen. Hierdurch wird die Oberfläche vergrößert und der Klebstoff kann sich zusätzlich formschlüssig in der Oberfläche verankern. Im Anschluss erfolgt die Reinigung der Waferviertel in einem Megaschallbad. Das Arbeitsprinzip für den Klebstoffauftrag entspricht der Abb. 44. Das Substrat wird auf der Montageplattform fixiert und die Rakeleinheit oberhalb positioniert. Die Rakelklinge wird mit dem Substrat in Kontakt gebracht. Die Schichtdicke wird über das Verfahren der Linearachse eingestellt und die Startposition für den Rakelprozess wird angefahren. Der Klebstoff wird über der gesamten Fläche ausreichend verteilt und die Montageplattform wird ohne Unterbrechung unterhalb der Rakelklinge verfahren. Anschließend wird das Klebstoffauftragswerkzeug seitlich weggeklappt und das Substrat zum Aushärten auf die Heizplatte gelegt.

Abb. 61 zeigt die erzielten Messergebnisse. Es ist erkennbar, dass sich die angestrebten Schichtdicken von 10, 20, 30, 40, 50, 75 und $100 \mu \mathrm{m}$ nicht genau als Abstandsmaß mit der Linearachse einstellen lassen. Sowohl der Messtaster als auch die Mikrometerstellschraube an der Linearachse selbst unterliegen einer gewissen Toleranz. Dennoch ist die maximale Abweichung von $2 \mu \mathrm{m}$ gegenüber den eingestellten Werten sehr zufriedenstellend. Anhand der Messergebnisse aus Abb. 61 (a) für den Zweikomponenten Klebstoff geht hervor, dass nach der Aushärtung alle Klebschichten auf der Oberfläche dünner sind als wie an der Linearachse eingestellt. Dies lässt auf eine Schrumpfung des Klebstoffes durch thermische Aushärtung schließen. Es ergibt sich ein Schwund zwischen 11 und $21 \%$ gegenüber der eingestellten Höhe an der Linearachse. Aus Abb. 61 (b) geht hervor, dass für den Einkomponenten Klebstoff keine Schrumpfung durch thermische Aushärtung erkennbar ist. Für beide Klebstoffe wird für Schichtdicken kleiner $40 \mu \mathrm{m}$ ein mittlerer absoluter Fehler von maximal $7 \mu \mathrm{m}$ gegenüber dem eingestellten Wert an der Linearachse erreicht. Nach Pfeifer (1982) gilt, die Klebschicht so dünn wie möglich aufzutragen. Betrachtet man hierzu im Detail für beide Klebstoffe einen Bereich von maximal $20 \mu \mathrm{m}$ Dicke, tritt lediglich ein mittlerer absoluter Fehler von maximal $3 \mu \mathrm{m}$ gegenüber der eingestellten Höhe an der Linearachse auf. Bei Schichtdicken des Zweikomponenten Klebstoffes von größer $50 \mu \mathrm{m}$ muss der Schwund beachtet werden.

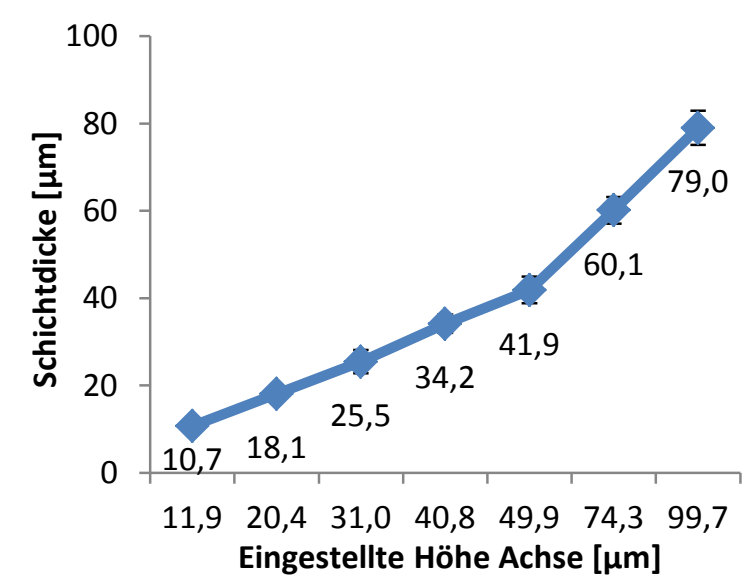

(a)

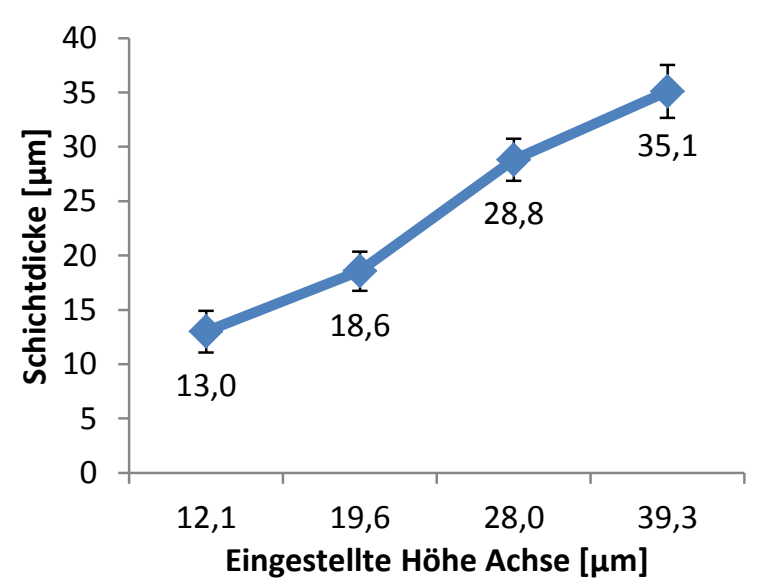

(b)

Abb. 61: Messergebnisse zu dem Klebstoffauftrag in (a) mit UHU PLUS ENDFEST 300 und in (b) mit DELO-MONOPOX ${ }^{\circledR}$ 6093. Messwerte entnommen aus Kagerer et al. (2013b). 
Neben der Einstellbarkeit der einzelnen Klebschichtdicken gilt es auch, deren Homogenität zu prüfen. Zu deren Beurteilung wird die Standardabweichung herangezogen. Für beide Klebstoffe ist die Standardabweichung bei Klebschichtdicken kleiner $40 \mu \mathrm{m}$ unter $3 \mu \mathrm{m}$. Selbst bei einer resultierenden Klebschichtdicke von $79 \mu \mathrm{m}$ für den Zweikomponenten Klebstoff ist diese noch unter $5 \mu \mathrm{m}$. Diese Werte lassen auf eine gute Homogenität über der gesamten Auftragsfläche schließen.

\subsubsection{Zusammenfassung der Ergebnisse}

Durch vorliegende Untersuchungen wird gezeigt, dass mit dem entworfenen Klebstoffauftragswerkzeug homogene Klebschichten auf Substraten aufgebracht werden können. Die Homogenität aller Schichtdicken wird durch die jeweiligen geringen Standardabweichungen belegt. Zudem hat sich ergeben, dass angestrebte Schichtdicken mit der vorhandenen Linearachse und des daran angebrachten Messtasters auf $\pm 2 \mu \mathrm{m}$ einstellbar sind. Für beide Klebstoffe wird in einem Schichtdickenbereich kleiner gleich $20 \mu \mathrm{m}$ ein mittlerer absoluter Fehler von maximal $3 \mu \mathrm{m}$ erzielt. Die Aufgabe aus Kapitel 4 wird erfüllt und der erste Teil der Hypothese in diesem Experiment wird bestätigt. Gerade dieser Bereich ist in Verbindung mit der Fügung von piezoelektrischen Wandlern mit Membranen bedeutend. Je dünner die Klebschicht ist, desto geringer ist die resultierende Biegesteifigkeit des Membranaktors. Im Ergebnis werden dadurch bei identischem Energieeintrag höhere Hübe des Aktors erzielt. Die Hypothese, Klebschichten größer 20 bis einschl. $40 \mu \mathrm{m}$ Dicke mit einem mittleren absoluten Fehler von maximal $6 \mu \mathrm{m}$ gegenüber der eingestellten Höhe an der Linearachse der Rakeleinheit auf Substraten zu erzielen, kann mit vorliegendem Experiment nur teilweise bestätigt werden. Für den Zweikomponenten Klebstoff beträgt bei einer angestrebten Schichtdicke von $40 \mu \mathrm{m}$ der mittlere absolute Fehler ca. $7 \mu \mathrm{m}$. Die restlichen Fehler liegen jedoch in dem vorgegebenen Bereich. Abb. 62 zeigt rasterelektronenmikroskopische Aufnahmen unterschiedlich dicker Klebschichten auf Substraten.

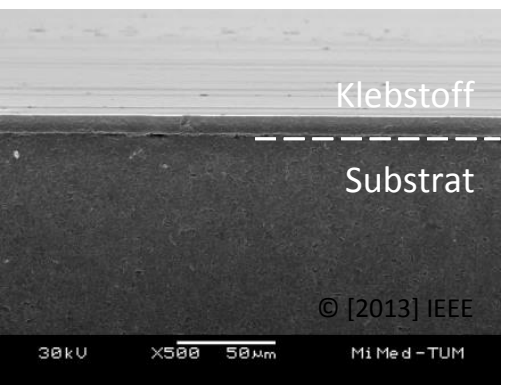

(a)

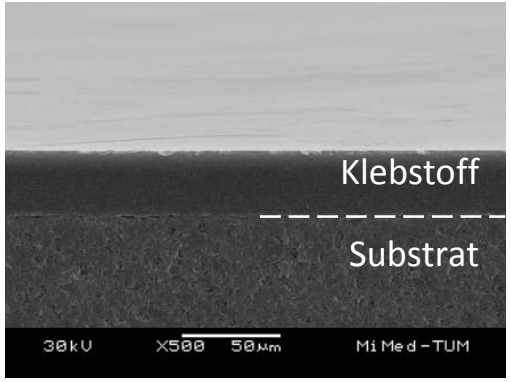

(d)

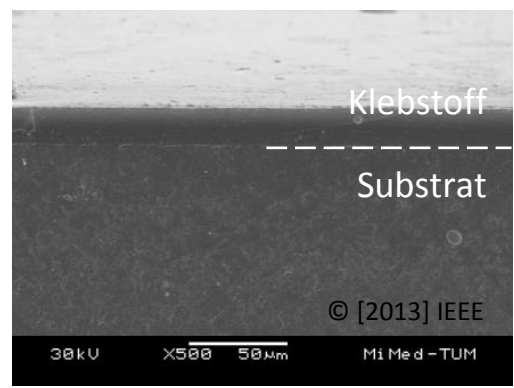

(b)

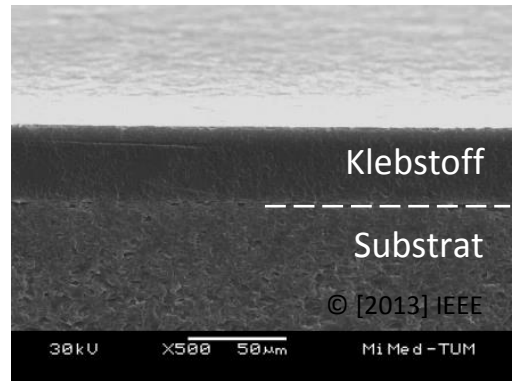

(e)

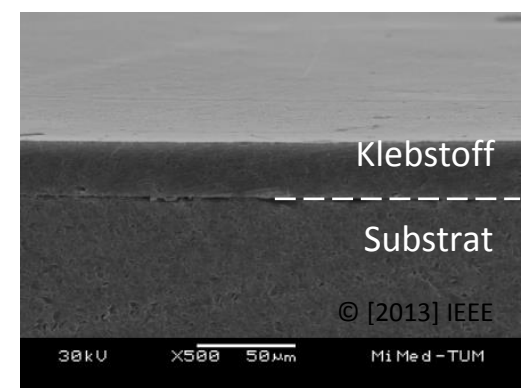

(c)

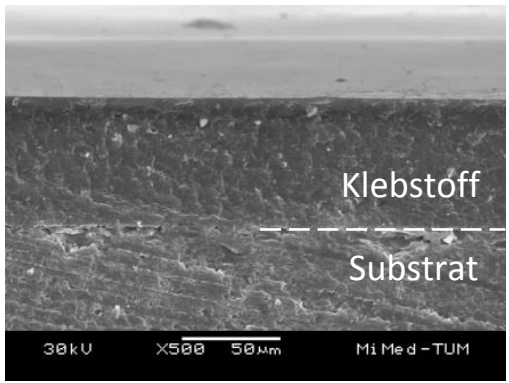

(f)

Abb. 62: Rasterelektronenmikroskopische Aufnahmen von Klebschichten auf Borosilikatglaswafern im Querschnitt (UHU PLUS ENDFEST 300) mit einer Dicke in (a) 10,7 $\mu \mathrm{m}$, (b) 18,1 $\mu \mathrm{m}$, (c) $25,5 \mu \mathrm{m}$, (d) $34,2 \mu \mathrm{m}$, (e) $41,9 \mu \mathrm{m}$ und (f) $60,1 \mu \mathrm{m}$. (a), (b), (c) und (e) entnommen aus Kagerer et al. (2013b); (d) und (f) entnommen aus Hüdig (2014). 


\section{Bedeutung der Ergebnisse}

Nach der Beschreibung des eigenen Ansatzes, der Realisierung und der notwendigen Experimente für die Mikrostrukturierung als auch -montage gilt es, die untersuchten Verfahren und Werkzeuge anzuwenden. Als Beispiel wird hierfür auf ein hybrides Mikrosystem, einen piezoelektrisch betriebenen Mikrotropfenerzeuger, zurückgegriffen.

Ziel ist es, diesen in einer ausreichenden Anzahl und in weniger als einem Tag (siehe Kapitel 4) zu fertigen, um Funktionsmodelle für eine Eigenschaftsbewertung in einem frühen Entwicklungsstadium in kurzer Zeit zu erhalten.

\subsection{Beschreibung eines planaren piezoelektrischen Mikrotropfenerzeugers}

\subsubsection{Aufbau des Mikrotropfenerzeugers}

Es wird auf einen Mikrotropfenerzeuger zurückgegriffen, für den ein Entwurf am Lehrstuhl für Mikrotechnik und Medizingerätetechnik zur Verfügung steht, der jedoch noch nicht erfolgreich umgesetzt bzw. charakterisiert ist. Dieser basiert auf einem piezoelektrisch betriebenen Membranaktor. Membransysteme zeichnen sich vor allem durch den Vorteil aus, dass das Fluid nicht direkt in Kontakt mit den elektrischen Komponenten des Aktors steht. Somit besteht auch die Möglichkeit zum Dosieren aggressiver und elektrisch leitfähiger Medien. Abb. 63 zeigt seinen Aufbau. Hauptbestandteil ist ein $525 \mu \mathrm{m}$ dicker Siliziumchip, welcher die fluidmechanischen Strukturen enthält. Über eine Fluidzuführung und eine Drossel wird das

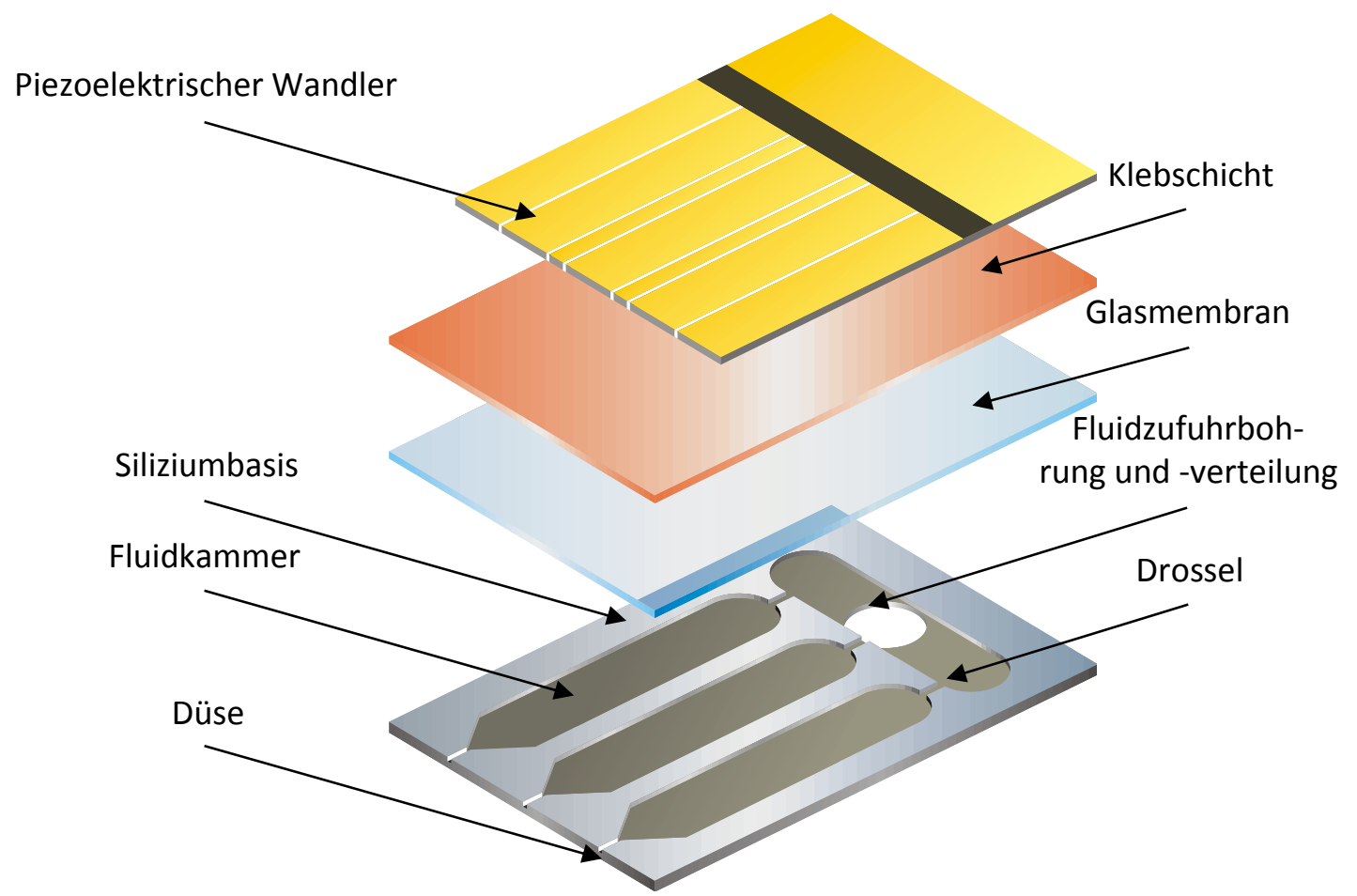

Abb. 63: Schematische Darstellung des membranbasierten Mikrotropfenerzeugers bestehend aus einem strukturierten piezoelektrischen Wandler mit zentralem Masseanschluss, einer Klebstoffschicht, einer Glasmembran und einer Siliziumbasis mit fluidmechanischen Strukturen (Fluidzuführung, Drossel, Fluidkammer und Düse). 
Fluid in eine Kammer gefördert. Auf der Oberseite des Siliziumchips ist eine Membran aus Borosilikatglas aufgebracht, welche die fluidmechanischen Strukturen abdichtet. So steht zu keinem Zeitpunkt das Fluid in Kontakt mit den elektrischen Komponenten des Aktors. Darüber hinaus zeigt sich der Aufbau als chemisch sehr resistent, da nur das Silizium und das Borosilikatglas mit dem Fluid in Kontakt kommen. Nur Fluide, die diese beiden Materialien chemisch angreifen, können nicht dosiert werden. Beispiele hierfür sind Kaliumhydroxid oder Flusssäuregemische.

In jedem Mikrotropfenerzeuger befinden sich drei Düsen. Hieraus ergibt sich der Vorteil, selbst wenn eine Düse blockiert ist, dass zwei weitere Düsen für Untersuchungen bereitstehen. Die Fluidkammern nehmen eine Breite von $2 \mathrm{~mm}$ und eine Länge von $10 \mathrm{~mm}$ ein. Die Tiefen der Kammern betragen mindestens $50 \mu \mathrm{m}$. Die Drossel besitzt eine Breite von $200 \mu \mathrm{m}$ bei einer Länge von $1 \mathrm{~mm}$ und die Fluidzufuhrbohrung hat einen Durchmesser von $2 \mathrm{~mm}$. Die minimale Düsenbreite beträgt $50 \mu \mathrm{m}$. Die Außenmaße eines solchen Siliziumchips sind $12 \times 18 \mathrm{~mm}^{2}$. Der Düsenkanal ist nur mit der durchsichtigen Glasmembran bedeckt. Hierdurch ist es möglich, den Tropfenbildungsprozess in der Düse zu beobachten und auch Funktionsstörungen, Partikelbildung / -blockierung etc. zu erkennen.

Ein strukturierter piezoelektrischer Wandler mit einer Dicke von $250 \mu \mathrm{m}$ ist über eine Klebstoffschicht mit der Membran verbunden. Für jede Pumpkammer wird eine Elektrodenzunge bereitgestellt. Der elektrische Stromkreis wird über einen zentralen Masseanschluss auf dem Wandler geschlossen. Der piezoelektrische Wandler bildet über die Klebstoffschicht mit der $100 \mu \mathrm{m}$ dicken Borosilikatglasmembran einen bimorphen Aktor. Über eine Klebstoffschicht wird der piezoelektrische Wandler auf die Membran aufgebracht. Wie aus Pfeifer (1982) zu entnehmen ist, ist es wichtig, diese Schicht so dünn wie möglich aufzutragen. Das Ergebnis ist eine reduzierte Biegesteifigkeit des Membranaktors, so dass höhere Hübe bei identischem elektrischem Energieeintrag das Ergebnis sind. Die Elektroden auf dem piezoelektrischen Wandler nehmen eine Breite von 1,6 mm und eine Länge von $10 \mathrm{~mm}$ ein. Die Außenmaße des gesamten Wandlers betragen $12 \times 16 \mathrm{~mm}^{2}$. Die einzelnen Elektrodenzungen werden durch komplett über der Dicke der piezoelektrischen Keramik abgetragenen Gebiete elektrisch voneinander getrennt. Es entstehen seitlich freistehende Elektrodenzungen.

Sowohl die vorgestellten Verfahren für die Mikrostrukturierung als auch der entwickelte Mikromontageplatz werden bei der Fertigung dieses Mikrotropfenerzeugers eingesetzt. Hierbei ergeben sich für den Laserstrahlmaterialabtrag folgende Anwendungen:

- Ebener Laserstrahlmaterialabtrag der fluidmechanischen Komponenten mit Ausnahme der Düsen in der Siliziumbasis. Dies sind die Pumpkammer, die Drossel und die Fluidverteilung

- Laserstrahlschneiden der Fluidzufuhrbohrung in dem Siliziumchip

Für das Trennschleifen mit einer Wafersäge ergeben sich folgende Anwendungen:

- Erstellung unterschiedlich breiter und tiefer Düsenkanäle in dem Siliziumchip

- Strukturierung der Elektroden und des zentralen elektrischen Masseanschlusses auf dem piezoelektrischen Wandler

Für den entwickelten Mikromontageplatz ergibt sich die Anwendung, die piezoelektrische Keramik über eine homogene und dünne $(\triangleq 20 \mu \mathrm{m})$ Klebstoffschicht zu fügen. 


\subsubsection{Funktionsweise des Mikrotropfenerzeugers}

Das Funktionsprinzip des vorliegenden Tropfenerzeugers basiert auf der Volumenverringerung einer fluidgefüllten Pumpkammer und wird in Abb. 64 gezeigt. Den Ausgangspunkt stellt die Füllung des Tropfenerzeugers mit dem zu dosierenden Fluid dar.

Wird ein elektrischer Puls an die jeweilige Elektrodenzunge angelegt, dehnt sich diese durch den piezoelektrischen Effekt senkrecht zur Oberfläche aus und wird gleichzeitig parallel zur Oberfläche gestaucht. Durch die starre Verbindung von passiver Membran und Wandler wölbt sich der bimorphe piezoelektrische Aktor nach innen und ruft eine plötzliche Volumenverringerung der fluidgefüllten Pumpkammer hervor. Hierdurch entsteht ein Druckimpuls, welcher einen Tropfen aus der Düse ausstößt. Je höher die entsprechende Biegung des bimorphen Aktors in die Kammer ist, desto höher ist der resultierende Druckimpuls. Die Drossel verhindert in diesem Schritt, dass der Druckimpuls in die Fluidzuführung entweicht.

Ein optionaler vorausgehender Schritt ist die Wölbung des Aktors nach oben durch Anlegen eines invertierten Pulses. Hierdurch wird der Meniskus im Düsenkanal zurückgezogen. Im Anschluss erfolgt der Wechsel der Polarität des Pulses und der bimorphe Aufbau wird in die Fluidkammer gedrückt und der Tropfenausstoß erfolgt. Vorteil einer vorherigen positiven und nachfolgenden negativen Wölbung ist eine höhere Dynamik des Systems. Jedoch erhöht sich hierdurch auch der Ansteueraufwand.

Nach dem Ende des elektrischen Pulses nimmt der Membranaktor wieder seine Ursprungslage ein. Dadurch erfolgt die Wiederbefüllung der Fluidkammer und der Tropfenerzeuger ist für den nächsten Tropfenausstoß einsatzbereit.

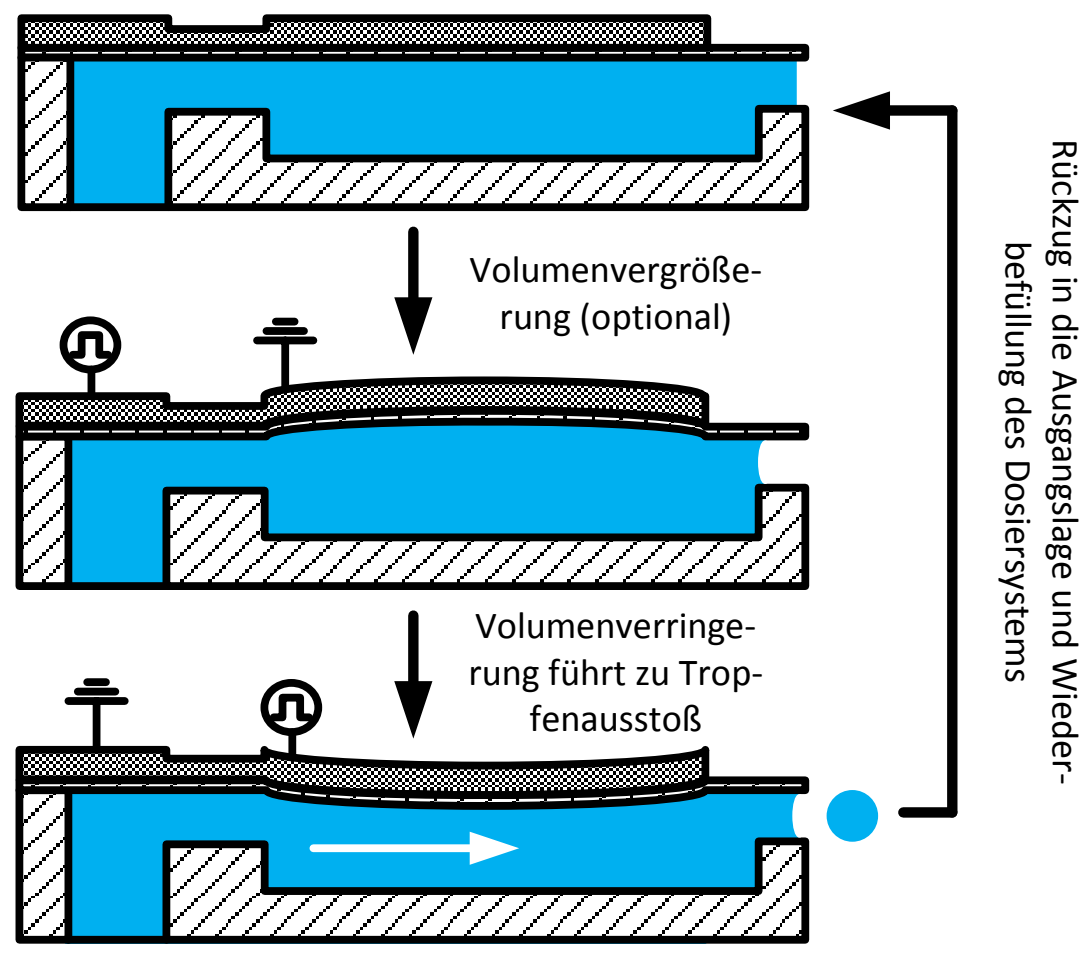

Abb. 64: Funktionsprinzip des membranbasierten Mikrotropfenerzeugers. Im Ausgangszustand ist die Kammer mit dem Fluid gefüllt und das System ist einsatzbereit. Eine optionale Biegung des Aktors nach oben bewirkt einen Meniskusrückzug in die Düse. Durch einen Polaritätswechsel des angelegten elektrischen Pulses biegt sich der Aktor in die Kammer und reduziert deren Volumen, wodurch ein Druckimpuls für den Tropfenausstoß entsteht. 


\subsection{Herstellung individueller Funktionsmodelle des Mikrotropfenerzeugers}

Anspruch an den Prozessflow ist es, eine ausreichende Anzahl an gleich gefertigten Funktionsmodellen in weniger als einen Tag bereitzustellen. Hierfür eignet sich die Batchherstellung, unter der in der Mikrotechnik die gleichzeitige Fertigung mehrerer Werkstücke verstanden wird. Der Vorteil ist, dass sowohl Zeit als auch Kosten für deren Fertigung eingespart werden. Das Ergebnis ist, dass für spätere fluidmechanische Untersuchungen eine ausreichende Anzahl gleich gefertigter Mikrotropfenerzeuger zur Verfügung steht.

\subsubsection{Prozessflow zur Herstellung der Mikrotropfenerzeuger}

Abb. 65 zeigt die Vorgehensweise zur Herstellung der Piezomembrandruckköpfe im Batchverfahren. Es werden gleichzeitig fünf Mikrotropfenerzeuger hergestellt. Dies bedeutet, dass für fluidmechanische Untersuchungen eine ausreichende Anzahl gleich gefertigter Druckköpfe mit 15 Düsen in Summe zur Verfügung gestellt wird. Ausgangsmaterial stellt je ein geviertelter 4 Zoll Silizium- und Borosilikatglaswafer dar. Das Silizium hat eine nominale Materialstärke von $525 \mu \mathrm{m}$ und das Borosilikatglas von $100 \mu \mathrm{m}$. Die Vierteilung der Wafer erfolgt mit der bereits in Kapitel 9 beschriebenen Wafersäge. Dieser Schritt kann durch die Verwendung von Wafern kleinerer Durchmesser eingespart werden, jedoch ist die Verwendung dieser Wafergrößen aufgrund des hohen Anschaffungspreises nicht wirtschaftlich umsetzbar. Die Dimensionen von Pumpkammer, Aktor, Drossel etc. sind in Kapitel 11.1 beschrieben.

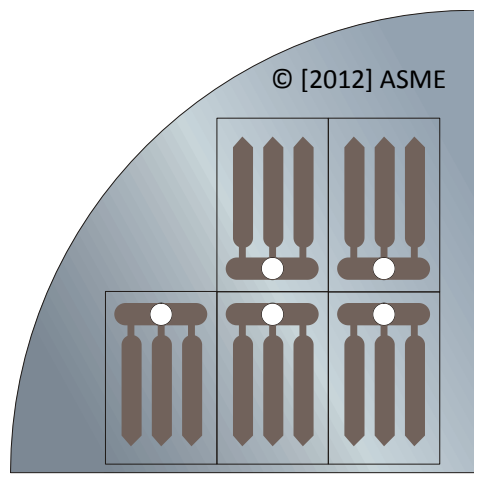

(a)

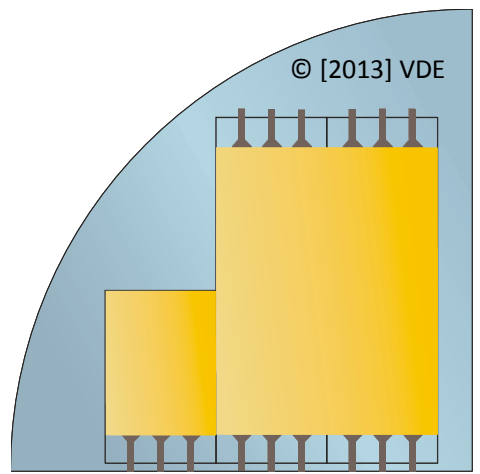

(d)

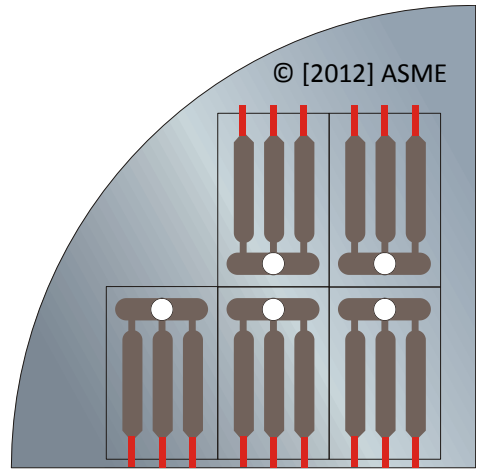

(b)

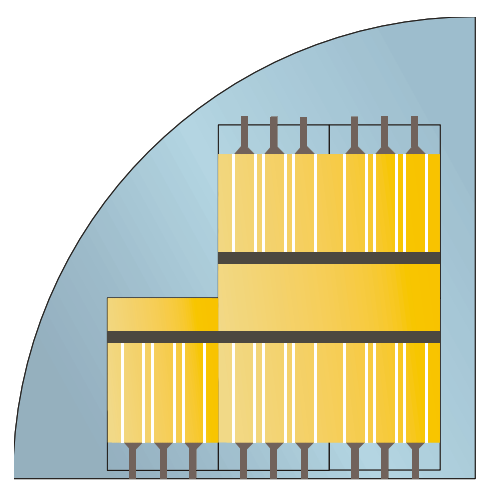

(e)

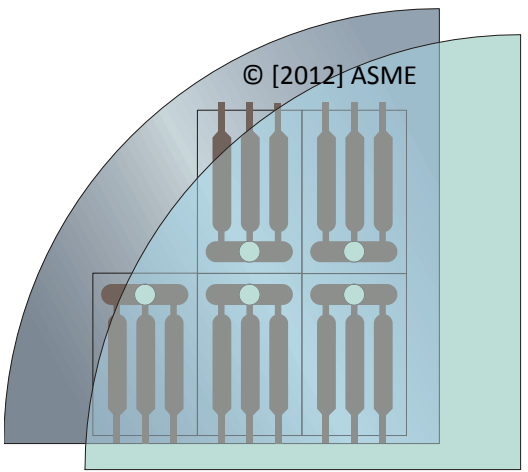

(c)

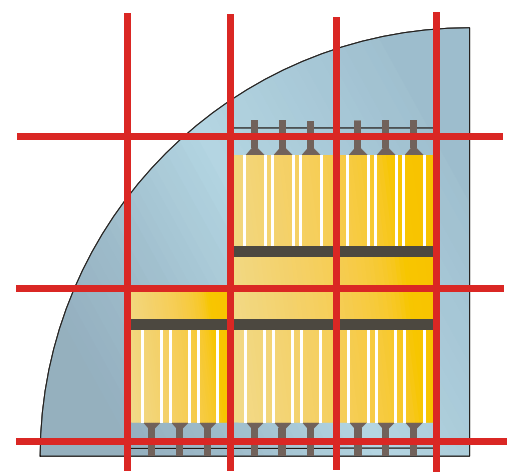

(f)

Abb. 65: Schematische Darstellung des Prozessflows. (a) Laserstrahlmaterialabtrag der fluidmechanischen Komponenten, (b) Schneiden / Trennschleifen der Düsenkanäle, (c) anodisches Bonden der Borosilikatglasmembran, (d) Fügung des piezoelektrischen Grundmaterials über eine Klebschicht, (e) Strukturierung des piezoelektrischen Grundmaterials und (f) Vereinzelung aus dem Verbund inkl. Schneiden der Länge der Düse. (a) bis (c) entnommen aus Kagerer et al. (2012a) und (d) aus Kagerer et al. (2013a). 


\subsubsection{Schritt 1: Mikrostrukturierung mittels Laserstrahlung}

Im ersten Schritt wird der Siliziumwafer mit dem Infrarot Lasersystem LS 2000 / 8000 der Firma Laser Systems (siehe Abb. 28 (b)) bei einer mittleren Leistung von $5 \mathrm{~W}$ strukturiert. Es werden die Pumpkammern, die Drosseln und die Fluidverteilung erstellt. Darüber hinaus werden Justagemarkierungen für spätere Fertigungsprozesse eingebracht und jeder Druckchip markiert. Es wird angestrebt, sowohl 50 als auch $100 \mu \mathrm{m}$ tiefe Strukturen zu erstellen. Wie aus Kapitel 10 als Ergebnis hervorgeht, wird der Fokus des Laserstrahls auf die Oberfläche des Siliziumwafers gelegt und ein Linienabstand der Schraffur von $25 \mu \mathrm{m}$ verwendet. Für $50 \mu \mathrm{m}$ tiefe Strukturen werden vier $\left(\mathrm{v}_{\mathrm{s}}=100 \mathrm{~mm} / \mathrm{s}\right)$ und für $100 \mu \mathrm{m}$ tiefe Strukturen acht $\left(\mathrm{v}_{\mathrm{s}}=125 \mathrm{~mm} / \mathrm{s}\right)$ Überfahrungen mit dem Laserspot gewählt. Der höhere Vorschub bei $100 \mu \mathrm{m}$ tief $\mathrm{zu}$ abladierenden Formen ergibt sich aus der notwendigen geringeren Abtragsrate gegenüber einem Vorschub von 100 mm/s. Da die Steuerungssoftware des Galvanometerscanners die komplexen Abtragsformen nicht generieren kann, werden diese mit dem CAD Programm CATIA V5 von Dassault Systèmes erstellt und als HPGL Datei in die Lasersteuerungssoftware importiert. Für das Ausschneiden der Fluidzufuhrbohrung werden zwei konzentrische Kreise mit einem Linienabstand von $25 \mu \mathrm{m}$ verwendet. Der Fokus wird auf der Oberfläche gelassen, statt mittig in das Werkstück nach den Ergebnissen aus Kapitel 10 zu legen. Dies spart ein zweites Verfahren der Höhenachse am Lasersystem und der Prozess kann in einem Schritt durchgeführt werden. Abb. 66 zeigt exemplarisch rasterelektronenmikroskopische Aufnahmen von laserabladierten fluidmechanischen Komponenten.

\subsubsection{Schritt 2: Mikrostrukturierung mittels Trennschleifen}

Im nächsten Schritt werden die Düsenkanäle mittels Trennschleifen mit der Wafersäge DAD321 von der Firma DISCO HI-TEC EUROPE eingebracht. Hierdurch werden senkrechte und vor allem glatte Düsenseitenwände erzielt. Das verwendete Sägeblatt FTB R46 45 x 100 von Mitsubishi Corporation entspricht den Anforderungen für Silizium aus Kapitel 7. Sowohl mit einer geringen Vorschubgeschwindigkeit von $5 \mathrm{~mm} / \mathrm{s}$ als auch mit einer hohen Spindeldrehzahl von $38000 \mathrm{U} / \mathrm{min}$ werden Schnitte mit geringem Chipping und geringer Welligkeit erreicht. Im Verlauf des Vorhabens werden drei unterschiedliche quadratische Düsen (Breite=Tiefe) gefertigt. Die Kantenlängen betragen jeweils 50, 70 und $100 \mu \mathrm{m}$. Wie aus Kapitel 10 hervorgeht, werden für eine $50 \mu \mathrm{m}$ breite Düse ein Schnitt, für eine $70 \mu \mathrm{m}$ breite Düse drei überlappende Schnitte und für eine $100 \mu \mathrm{m}$ breite Düse fünf überlappende Schnitte benötigt. Der Versatz beträgt hierbei 12,5 bzw. 13,5 $\mu \mathrm{m}$. Die Länge eines Düsenkanals ist auf $2 \mathrm{~mm}$ ausgelegt. Es wird immer aus der jeweiligen Pumpkammer hinaus in das Vollmaterial geschnitten. Die Bestimmung der Nulllage der Oberfläche, um im Anschluss die Tiefe des

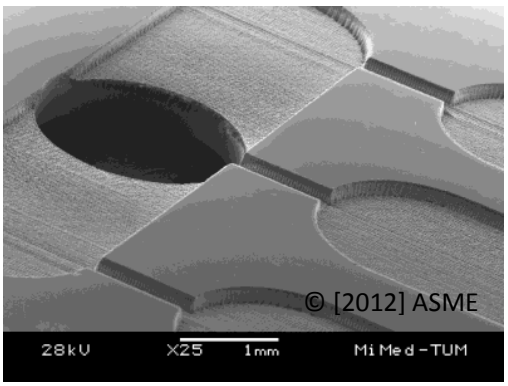

(a)

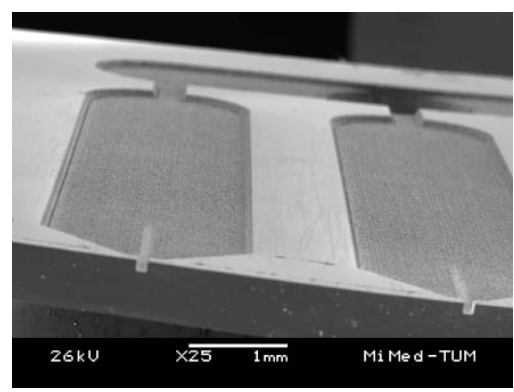

(b)

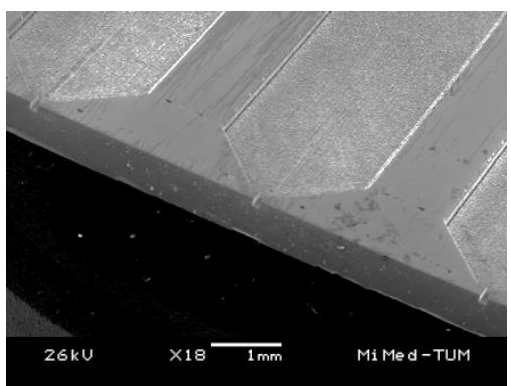

(c)

Abb. 66: Rasterelektronenmikroskopische Aufnahmen von laserabladierten fluidmechanischen Komponenten in Silizium. (a) Fluidverteilung, (b) Blick auf die Pumpkammern und (c) Düsenarray. (a) entnommen aus Kagerer et al. (2012a). 
Schnittes zu definieren, erfolgt mittels des vorgestellten Verfahrens aus Kapitel 7. Hierbei wird vor dem eigentlichen Schnitt das Sägeblatt an einer nicht funktionstragenden Stelle auf dem Chip mikrometerweise herabgesenkt, bis eine leichte Kerbe auf der Oberfläche erkennbar ist. Abb. 67 zeigt rasterelektronenmikroskopische Aufnahmen von Düsen mit unterschiedlichen Breiten.

\subsubsection{Schritt 3: Verbindung von Membran und Substrat}

Der dritte Schritt ist die Verbindung von Silizium mit dem Borosilikatglas durch anodisches Bonden. Es wird ein nur $100 \mu \mathrm{m}$ dickes Borosilikatglas (Borofloat $33^{\circledR}$ der Firma Plan Optik) verwendet, damit die Biegesteifigkeit des bimorphen Aktors reduziert wird (Pfeifer, 1982). Dickere Membranen würden eine höhere Biegesteifigkeit hervorrufen, welche wiederum den Hub bzw. das verdrängte Kammervolumen senken werden. Dadurch wird dem Tropfenbildungsprozess weniger Energie zur Verfügung gestellt und Tropfen hochviskoser Medien können eventuell nicht ausgegeben werden. Beide Komponenten werden vor dem anodischen Bonden umfangreich gereinigt. Hierfür werden die Oberflächen mit Lichtwellenleiterläppfolie sowohl mit 12 als auch mit $1 \mu \mathrm{m}$ Körnung geläppt und anschließend sowohl in einem Ultraals auch in einem Megaschallbad in Aceton gereinigt, so dass sich keine Partikel mehr auf den Oberflächen befinden. Beide Substrate werden in den Bondplatz eingelegt, auf $450{ }^{\circ} \mathrm{C}$ aufgewärmt und bei einer elektrischen Spannung von $1,2 \mathrm{kV}$ miteinander anodisch gebondet.

\subsubsection{Schritt 4: Mikromontage von piezoelektrischer Keramik und Membran}

Im Anschluss wird die piezoelektrische Keramik VIBRIT 1876 von Johnson Matthey Catalysts (Germany) auf die Membran geklebt. Das Ausgangsmaterial der Keramik hat die Maße $46,9 \times 25 \mathrm{~mm}^{2}$ und ist bereits bei Lieferung mit dem Elektrodenmaterial (Gold) versehen. Mit der bereits beschriebenen Wafersäge wird die Keramikplatte in zwei Teile geschnitten, so dass diese den Dimensionen der geviertelten Wafer angepasst werden. Die Materialdicke der Keramik beträgt $0,25 \mathrm{~mm}$. Vor dem Fügeprozess wird die Polarität der piezoelektrischen Keramik geprüft. Hierzu werden die Tastspitzen eines Voltmeters unter leichtem Druck aufgelegt und die Richtung der Potentialdifferenz bestimmt. DIN EN 13887 (Stand November 2003) empfiehlt, vor dem Klebeprozess die Oberfläche aufzurauen, um die Leistungsfähigkeit der Klebeverbindung zu erhöhen. Hierdurch wird die Oberfläche vergrößert und der Klebstoff kann sich zusätzlich formschlüssig in der Oberfläche verankern. Die piezoelektrische Keramik weist von Natur aus eine raue Oberfläche auf. Daher wird nur das Borosilikatglas mit Lichtwellenleiterläppfolie mit $30 \mu \mathrm{m}$ Körnung kurz angeschliffen. Im Anschluss erfolgt die Reinigung beider Fügepartner mit dem Reiniger DELOTHEN ${ }^{\circledR}$ EP von DELO Industrie Kleb-

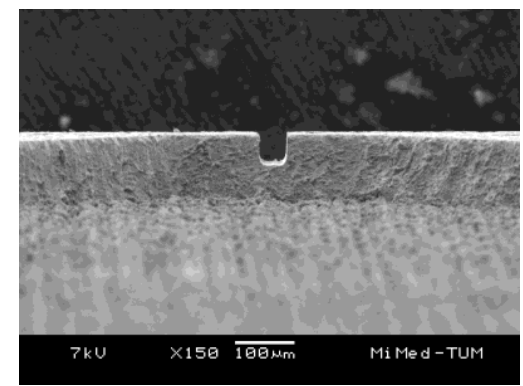

(a)

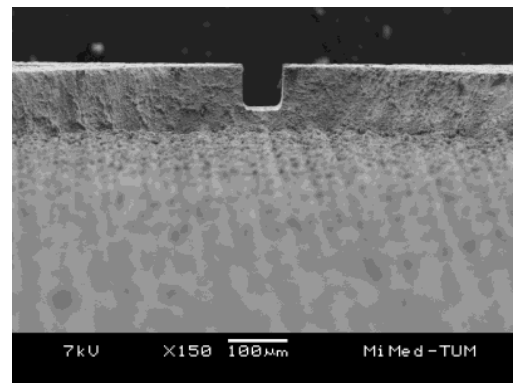

(b)

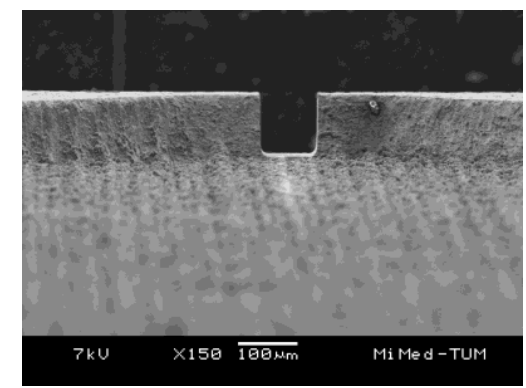

(c)

Abb. 67: Rasterelektronenmikroskopische Aufnahmen von Düsen, hergestellt durch Trennschleifen mit der Wafersäge mit Blick aus der Pumpkammer. Die Breite entspricht der Tiefe in (a) $50 \mu \mathrm{m}$, (b) $70 \mu \mathrm{m}$ und (c) $100 \mu \mathrm{m}$. Entnommen aus Meeuw (2014). 
stoffe, um Verunreinigungen etc. zu entfernen. Der Klebstoff UHU PLUS ENDFEST 300 von UHU wird in einem Verhältnis von 1:1 (Härter zu Binder) angemischt. Zur Erzielung homogener und definierter Klebschichten auf dem Borosilikatglas wird der in Kapitel 9 beschriebene Mikromontageplatz mit der angebrachten Klebstoffrakeleinheit eingesetzt. Die Bedienung des Mikromontageplatzes erfordert eine geringe Einarbeitungszeit. Es wird eine $20 \mu \mathrm{m}$ dicke Klebschicht aufgebracht. Im Anschluss werden die beiden Teile der piezoelektrischen Keramik auf das Borosilikatglas abgesetzt. Die Aushärtung des Klebstoffs erfolgt unter Temperatureinwirkung bei $70{ }^{\circ} \mathrm{C}$ für $45 \mathrm{~min}$, um zum einen die Aushärtezeit zu reduzieren und zum anderen die Festigkeit gegenüber Raumtemperaturaushärtung zu erhöhen (UHU, 2015). Abb. 68 zeigt rasterelektronenmikroskopische Aufnahmen der gefügten Bauteile.

\subsubsection{Schritt 5: Strukturierung der Elektroden mittels Trennschleifen}

Der fünfte Schritt in der Fertigung ist die Strukturierung von sowohl der Elektrodenzungen als auch der zentralen Masse mit der Wafersäge. Ziel ist es, freitragende Elektrodenzungen (Elektrodenmaterial: Gold) mit einer Breite von 1,6 mm zu generieren. Es wird ein Universalsägeblatt der Firma Microkerf (Modell 2.187-8-15 NiF5-N01) gewählt, welches nicht nur für die Strukturierung der piezoelektrischen Keramik, sondern auch für die Vereinzelung der Chips aus der Waferebene heraus für alle drei Materialien im nachfolgenden Schritt geeignet ist. Das Universalsägeblatt besitzt eine Breite von $0,2 \mathrm{~mm}$, eine Körnung von $15 \mu \mathrm{m}$, einen Blattüberstand von 1,67 mm und eine mittlere weiche Nickelbindung. Die Strukturierung der Elektrodenzungen und der zentralen elektrischen Masse erfolgt mit einem Vorschub von $7 \mathrm{~mm} / \mathrm{s}$ bei einer Spindeldrehzahl von $38000 \mathrm{U} / \mathrm{min}$. Es werden auch hier wiederum überlappende Schnitte eingesetzt. Abb. 69 zeigt rasterelektronenmikroskopische Aufnahmen des strukturierten piezoelektrischen Wandlers. Die Trennung von Masse zu Elektroden ist ca. $30 \mu \mathrm{m}$ tief abgetragen und besitzt lediglich die Funktion, die beiden Teile elektrisch voneinander zu isolieren.

\subsubsection{Schritt 6: Vereinzelung mittels Trennschleifen}

Der letzte Schritt in dem vorgestellten Prozessflow ist zweigeteilt. Im ersten Teilschritt werden die fünf einzelnen Druckchips aus dem geviertelten Wafer herausgetrennt. Hierbei wird auf das o. g. Universalsägeblatt zurückgegriffen. Insgesamt werden hierzu fünf Schnitte benö-

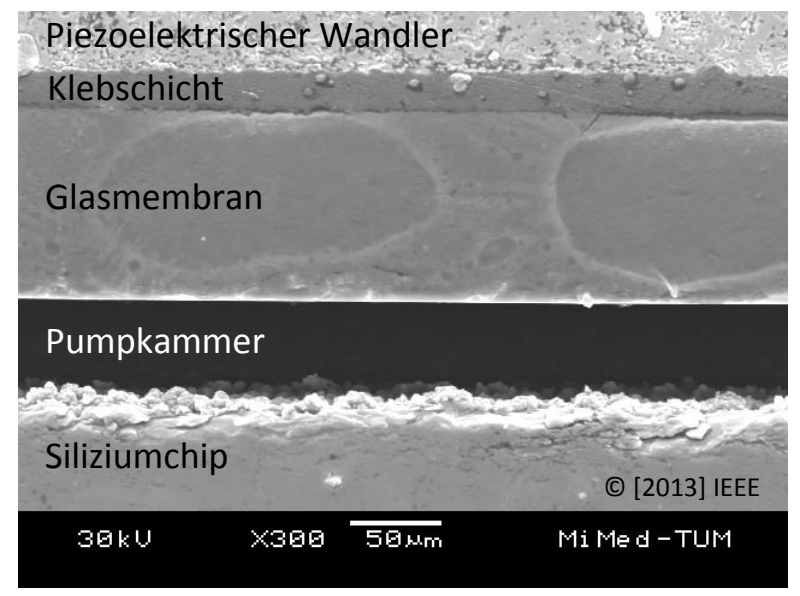

(a)

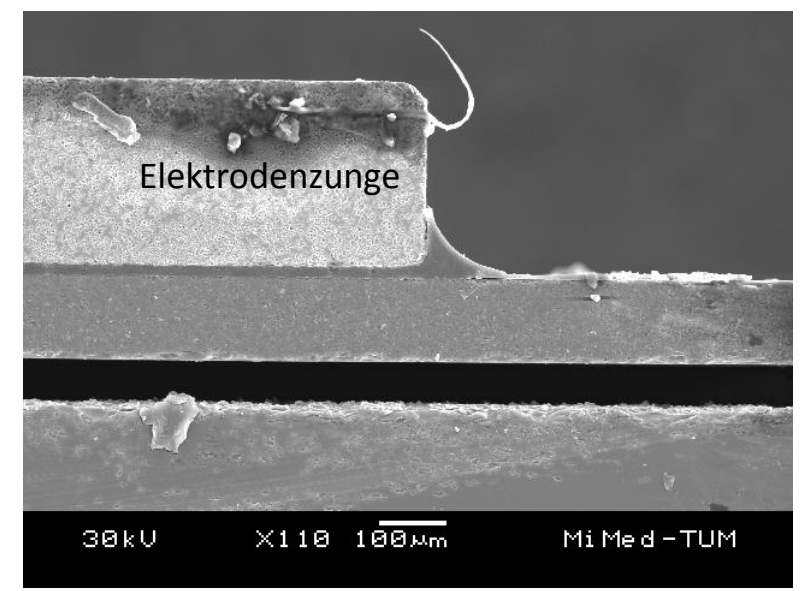

(b)

Abb. 68: Rasterelektronenmikroskopische Aufnahmen der verklebten piezoelektrischen Keramik mit der Borosilikatglasmembran. (a) Detailaufnahme im Querschnitt und (b) Aufnahme einer Aktorelektrodenzunge. (a) entnommen aus Kagerer et al. (2013b). 
tigt. Dieser Schritt wird direkt im Anschluss an die Strukturierung der Elektroden durchgeführt. Im zweiten Teil werden die Düsenlängen auf ihr Sollmaß geschnitten. Hierfür werden nach der Vereinzelung die einzelnen Druckchips erneut auf die Sägefolie geklebt. Da die Düse einen essentiellen Einfluss auf die Tropfenbildung besitzt, ist es von Nöten, neben der Breite und der Tiefe auch die Länge exakt zu schneiden. Daher wird zum Schneiden der Düsenlänge auf das feine Silizium Sägeblatt mit kleiner Körnung auf Kosten der Blattstandzeit zurückgegriffen. Dieser Schritt im Prozessflow ist der erste, in dem nach der Verkapselung der Düsenkanäle mittels anodischem Bonden die Düse wieder freigelegt wird. Um der Gefahr entgegenzuwirken, große Glaspartikel über das Prozesswasser in den Düsenkanal einzubringen, erfolgt eine Rückdünnung des Borosilikatglases bis $10 \mu \mathrm{m}$ oberhalb des Düsenkanals. In einem zweiten Schnitt werden das restliche Borosilikatglas und das Silizium durchtrennt. Durch diesen zusätzlichen Rückdünnungsvorgang werden weniger Partikel freigesetzt und das Risiko, offengelegte Düsenkanäle mit Partikeln zu blockieren, reduziert.

Während der gesamten Fertigung sind neben den oben genannten Hauptschritten auch Zwischenschritte notwendig. Diese sind bspw. die Spülung / Reinigung von Oberflächen, die Messung der Dimensionen einzelner Komponenten, das Aufbringen von Werkstiucken auf Sägefolie oder das Dressen von Sägeblättern für die Wafersägenbearbeitung. Es wird durchgehend vom ersten bis zum letzten Schritt der Vereinzelung auf der kostengünstigen Waferebene gearbeitet. Insgesamt stehen mit vorliegendem Prozessflow fünf gleich gefertigte Druckchips respektive 15 Düsen für Experimente zur Verfügung.

\subsubsection{Bewertung und Gegenüberstellung des Prozesses}

Eines der übergeordneten Ziele dieser Arbeit ist es, vorliegenden Mikrotropfenerzeuger in weniger als einen Tag zu fertigen, um ein mögliches Redesign kurzfristig bereitzustellen. Tabelle 3 zeigt die benötigte Zeit, um fünf Druckchips mit dem vorgestellten Prozessflow zu fertigen. Es werden alle sechs Hauptschritte für die Fertigung von unterschiedlichen Varianten von Druckköpfen dargestellt. Dabei werden die fluidmechanischen Strukturen 50 und $100 \mu \mathrm{m}$ tief mittels Laserstrahlung abgetragen und die Düsen werden zwischen 50 und $100 \mu \mathrm{m}$ Kantenlänge gefertigt. Es werden zwei unterschiedliche Zeiten betrachtet:

1. Die reine Prozesszeit für die sechs Hauptschritte

2. Die gesamte Fertigungszeit, welche sich aus der Prozesszeit, der Arbeitszeit von Operatoren und eventuellen Wartezeiten, wie bspw. Aufheiz- oder Abkühlvorgänge, zusammensetzt

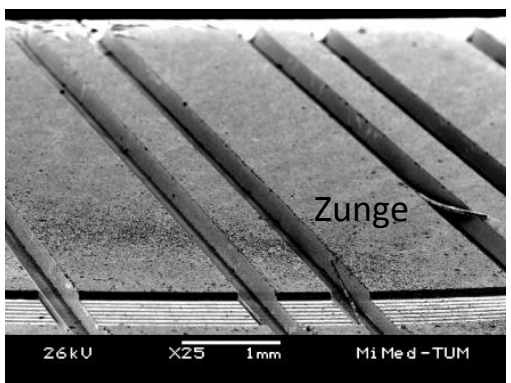

(a)

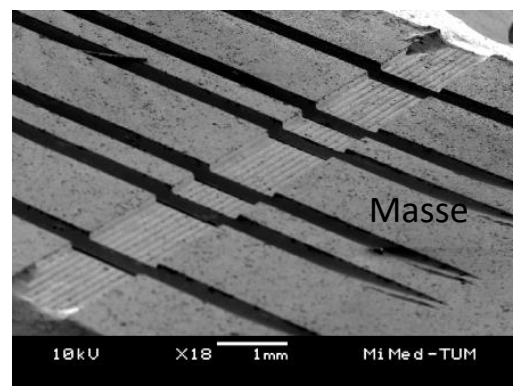

(b)

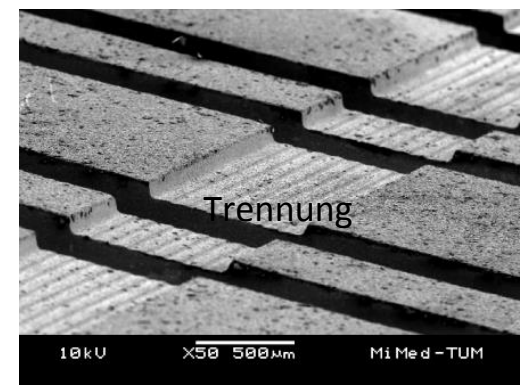

(b)

Abb. 69: Rasterelektronenmikroskopische Aufnahmen des strukturierten piezoelektrischen Wandlers auf der Glasmembran. (a) 1,6 mm breite Aktorelektrodenzungen mit seitlicher Freilegung, (b) zentraler elektrischer Masseanschluss und (c) oberflächliche Trennung von Elektrodenzungen zu zentraler elektrischer Masse. 
Tabelle 3: Fertigungszeit für fünf Druckchips. Die Zeiten sind jeweils auf volle Minuten gerundet.

\begin{tabular}{|c|c|c|c|}
\hline Schritt & Nr. & Beschreibung & Zeit [min] \\
\hline \multirow{4}{*}{$\begin{array}{l}\text { Laserstrahl- } \\
\text { materialbe- } \\
\text { arbeitung }\end{array}$} & 1.1 & Vorbereitung (Laserleistung messen, Positionierung) & 3 \\
\hline & $1.2(\mathrm{a})$ & Prozess: $50 \mu \mathrm{m}$ tiefe Komponenten in Silizium & 31 \\
\hline & $1.2(\mathrm{~b})$ & Prozess: $100 \mu \mathrm{m}$ tiefe Komponenten in Silizium & 38 \\
\hline & 1.3 & Nachbereitung (Beschriften, Läppen, Reinigen) & 14 \\
\hline \multirow{5}{*}{$\begin{array}{l}\text { Düsenferti- } \\
\text { gung }\end{array}$} & 2.1 & $\begin{array}{l}\text { Vorbereitung (Aufbringen auf Sägefolie, Höhe messen, Säge- } \\
\text { blatt dressen, Blattdicke bestimmen) }\end{array}$ & 7 \\
\hline & 2.2 (a) & $\begin{array}{l}\text { Prozess: Düsenkanal schneiden mit Kantenlänge } 50 \mu \mathrm{m} \\
\text { (Ausrichten, Oberfläche bestimmen, Positionieren) }\end{array}$ & 23 \\
\hline & $2.2(\mathrm{~b})$ & $\begin{array}{l}\text { Prozess: Düsenkanal schneiden mit Kantenlänge } 70 \mu \mathrm{m} \\
\text { (Ausrichten, Oberfläche bestimmen, Positionieren) }\end{array}$ & 24 \\
\hline & $2.2(\mathrm{c})$ & $\begin{array}{l}\text { Prozess: Düsenkanal schneiden mit Kantenlänge } 100 \mu \mathrm{m} \\
\text { (Ausrichten, Oberfläche bestimmen, Positionieren) }\end{array}$ & 27 \\
\hline & 2.3 & Nachbereitung (UV Belichtung) & 30 \\
\hline & & & \\
\hline \multirow{5}{*}{$\begin{array}{l}\text { Anodisches } \\
\text { Bonden }\end{array}$} & 3.1 & Vorbereitung (Läppen, Reinigen) & 47 \\
\hline & 3.2 & Aufheizen der Bondanlage auf $450{ }^{\circ} \mathrm{C}$ & 35 \\
\hline & 3.3 & Prozess: Anodisches Bonden $\left(0,4 \mathrm{~mA} ; 1,2 \mathrm{kV} ; 450{ }^{\circ} \mathrm{C}\right)$ & 45 \\
\hline & 3.4 & Abkühlen der Bondanlage $\left(<40^{\circ} \mathrm{C}\right)$ & 90 \\
\hline & 3.5 & Nachbereitung (Fluidzuführung mit Klebefilm versiegeln) & 1 \\
\hline \multirow{5}{*}{$\begin{array}{l}\text { Klebepro- } \\
\text { zess }\end{array}$} & 4.1 & $\begin{array}{l}\text { Vorbereitung (Läppen, Reinigen, Klebstoff mischen, Positio- } \\
\text { nierung Rakeleinheit) }\end{array}$ & 7 \\
\hline & 4.2 & Prozess: Fügung von Membran mit Keramik (Rakelvorgang) & 4 \\
\hline & 4.3 & $\begin{array}{l}\text { Prozess: Klebstoffaushärtung bei } 70{ }^{\circ} \mathrm{C} \text { für } 45 \text { min inkl. Auf- } \\
\text { heizen der Heizplatte auf } 70^{\circ} \mathrm{C}\end{array}$ & 50 \\
\hline & 4.4 & Abkühlen der Heizplatte $\left(<40^{\circ} \mathrm{C}\right)$ & 40 \\
\hline & 4.5 & Nachbereitung (Läppen, Reinigen) & 2 \\
\hline \multirow{4}{*}{$\begin{array}{l}\text { Strukturie- } \\
\text { rung des } \\
\text { piezoelektri- } \\
\text { schen Wand- } \\
\text { lers und } \\
\text { Vereinze- } \\
\text { lung }\end{array}$} & 5.1 & $\begin{array}{l}\text { Vorbereitung (Aufbringen auf Sägefolie, Höhe messen, Säge- } \\
\text { blatt dressen, Blattdicke bestimmen) }\end{array}$ & 7 \\
\hline & 5.2 & $\begin{array}{l}\text { Prozess: Strukturierung der Aktorzungen (Breite: 1,6 mm) und } \\
\text { der zentralen elektrischen Masse }\end{array}$ & 16 \\
\hline & 5.3 & Prozess: Vereinzelung der Chips aus Waferebene & 6 \\
\hline & 5.4 & Nachbereitung (UV Belichtung) & 30 \\
\hline
\end{tabular}




\begin{tabular}{|c|c|c|c|}
\hline Schritt & Nr. & Beschreibung & Zeit [min] \\
\hline \multirow{3}{*}{$\begin{array}{l}\text { Düsenlänge } \\
\text { schneiden }\end{array}$} & 6.1 & $\begin{array}{l}\text { Vorbereitung (Aufbringen auf Sägefolie, Höhe messen, Säge- } \\
\text { blatt dressen, Blattdicke bestimmen) }\end{array}$ & 7 \\
\hline & 6.2 & Prozess: Schneiden der Düsenlänge inkl. Rückdünnung & 9 \\
\hline & 6.3 & Nachbereitung (UV Belichtung) & 30 \\
\hline \multicolumn{3}{|c|}{$\begin{array}{l}\text { Prozesszeit ( } 50 \mu \mathrm{m} \text { tiefe Komponenten } \& 50 \mu \mathrm{m} \text { Düse) } \\
\text { (Nr. } 1.2 \text { (a) \& } 2.2 \text { (a) \& } 3.3 \& 4.2 \& 4.3 \& 5.2 \& 5.3 \& 6.2 \text { ) }\end{array}$} & 184 \\
\hline \multicolumn{3}{|c|}{$\begin{array}{l}\text { Prozesszeit (100 } \mu \mathrm{m} \text { tiefe Komponenten \& } 100 \mu \mathrm{m} \text { Düse) } \\
\text { (Nr. } 1.2 \text { (b) \& } 2.2 \text { (c) \& } 3.3 \& 4.2 \& 4.3 \& 5.2 \& 5.3 \& 6.2)\end{array}$} & 195 \\
\hline \multicolumn{3}{|c|}{ Gesamtzeit (50 $\mu \mathrm{m}$ tiefe Komponenten \& $50 \mu \mathrm{m}$ Düse) } & 534 \\
\hline \multicolumn{3}{|c|}{ Gesamtzeit (100 $\mu \mathrm{m}$ tiefe Komponenten \& $100 \mu \mathrm{m}$ Düse $)$} & 545 \\
\hline
\end{tabular}

Aus Tabelle 3 gehen beispielshaft die Fertigungszeiten sowohl für Druckchips mit 50 als auch mit $100 \mu \mathrm{m}$ tief abgetragenen Strukturen hervor. Erwartungsgemäß werden die Druckköpfe mit $50 \mu \mathrm{m}$ breiten Düsen und mit $50 \mu \mathrm{m}$ tief laserabladierten Strukturen in der kürzesten Zeit hergestellt. Die reine Prozesszeit beträgt hierbei 184 Minuten. Die Gesamtzeit beträgt 534 Minuten. Die Druckköpfe mit $100 \mu \mathrm{m}$ breiten Düsen und mit $100 \mu \mathrm{m}$ tief laserabladierten Strukturen benötigen 195 Minuten reine Prozesszeit bzw. 545 Minuten Gesamtzeit für ihre Fertigung.

In weniger als einen Tag werden fünf gleich gefertigte Druckchips hergestellt. Die gestellte Aufgabe hinsichtlich der Herstellungszeit aus Kapitel 4 ist damit erfüllt. Es zeigt sich an dieser Stelle, welch immens wichtigen Stellenwert gezielt angepasste Werkzeuge, wie bspw. die Laserstrahlmaterialbearbeitung, für die Fertigung von Funktionsmodellen der Mikrosystemtechnik haben. Somit ist es möglich, notwendige Änderungen am Mikrosystem in kurzer Zeit zu verifizieren, eine Variantenvielfalt kurzfristig bereitzustellen und den Entwicklungsprozess wirtschaftlich zu gestalten. Vergleicht man die reine Prozesszeit der sechs Hauptschritte mit der Gesamtzeit, ergibt sich, dass die reine Prozesszeit ca. ein Drittel der Gesamtzeit beträgt. Dies bedeutet, dass zwei Drittel der Zeit für Rüstvorgänge, wie bspw. Messung der Laserleistung, Ausrichtung von Bauteilen oder auch Aufheizvorgänge, benötigt werden.

Eine weitere Aufgabe vorliegender Arbeit ist es nach Kapitel 4, die individuellen Funktionsmodelle nicht nur in kurzer Zeit, sondern auch mit einer hohen Formtreue zu fertigen. Nach der Aufgabenstellung müssen Freiformen mit einem maximalen absoluten Fehler von $10 \mu \mathrm{m}$, Kanäle respektive Düsen mit variierbarem Querschnitt von $5 \mu \mathrm{m}$, der Klebschichtauftrag auf Substraten von $3 \mu \mathrm{m}$ (bis zu einer Dicke von $20 \mu \mathrm{m}$ ) und die Positionierung zweier Bauteile von $20 \mu \mathrm{m}$ realisiert werden. In Kapitel 10 sind bereits die Nachweise zur Erfüllung der Aufgabenstellung bzgl. der Klebschichtdicke, der Positionierung und der Fertigung von senkrechten Kanälen mit variierbarer Breite und Tiefe erbracht.

Aus den Abb. 70 und Abb. 71 gehen die weiteren Nachweise hervor. Abb. 70 (a) zeigt den Nachweis bzgl. des Laserstrahlabtrages in Silizium für 50 und $100 \mu \mathrm{m}$ tief abgetragene Fluidkammern. Die jeweils dargestellten Mittelwerte werden aus mindestens 285 Messwerten er- 
hoben, welche wiederum aus mindestens 19 Fertigungschargen über einen Zeitraum von mindestens neun Monaten stammen. Abb. 70 (b) zeigt die Ergebnisse für die Erstellung unterschiedlicher Düsenlängen. Insbesondere zählen die Düsen zu den wichtigsten Bestandteilen eines Tropfenerzeugers und erfordern daher eine hohe Formtreue. Der sich ergebende Mittelwert, bspw. der $100 \mu \mathrm{m}$ langen Düse stammt aus über 160 Messwerten aus 15 Fertigungschargen über einen Zeitraum von 15 Monaten.

In Abb. 70 (c) sind die Ergebnisse für unterschiedlich breite Elektroden des piezoelektrischen Aktors dargestellt. Für die Standard Elektrodenbreite von 1,6 mm werden über 60 Messwerte aus 14 Fertigungschargen über einen Zeitraum von 15 Monaten für die Mittelwertsbildung herangezogen. Alle Elektrodenbreiten liegen in der geforderten Genauigkeitsklasse aus Kapitel 4. Abb. 70 (d) zeigt die Ergebnisse zu der Fertigung von drei unterschiedlich breiten Drosseln. Für die Standard Drosselbreite von $200 \mu \mathrm{m}$ wird der Mittelwert aus über 180 Messwerten von 17 Fertigungschargen über einen Zeitraum von 24 Monaten gebildet.

Es wird an dieser Stelle festgehalten, dass bei der Fertigung des Mikrotropfenerzeugers alle geforderten Genauigkeitsangaben aus Kapitel 4 eingehalten werden.

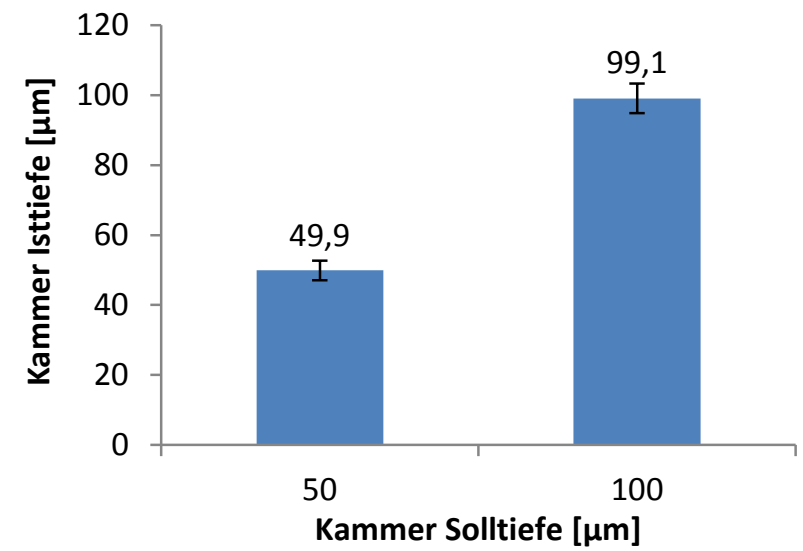

(a)

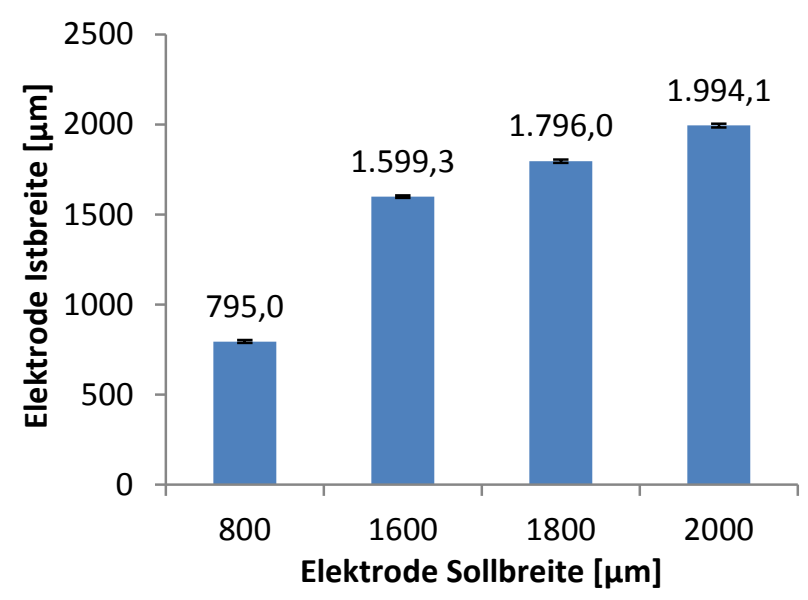

(c)

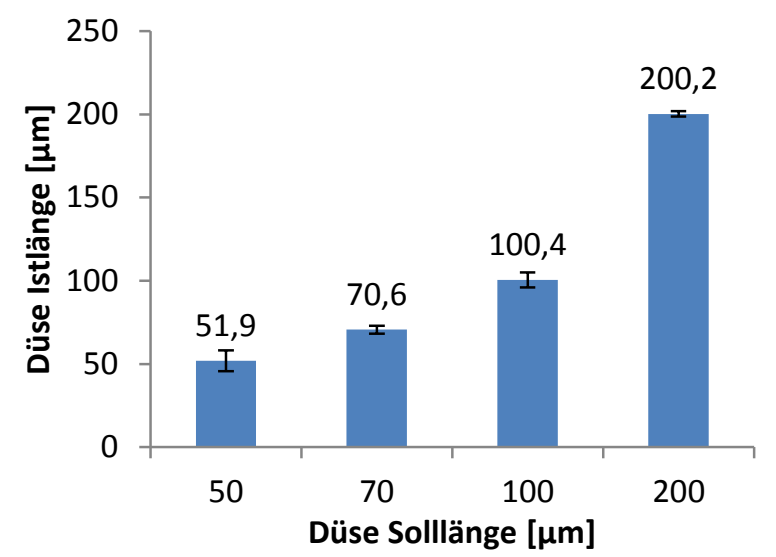

(b)

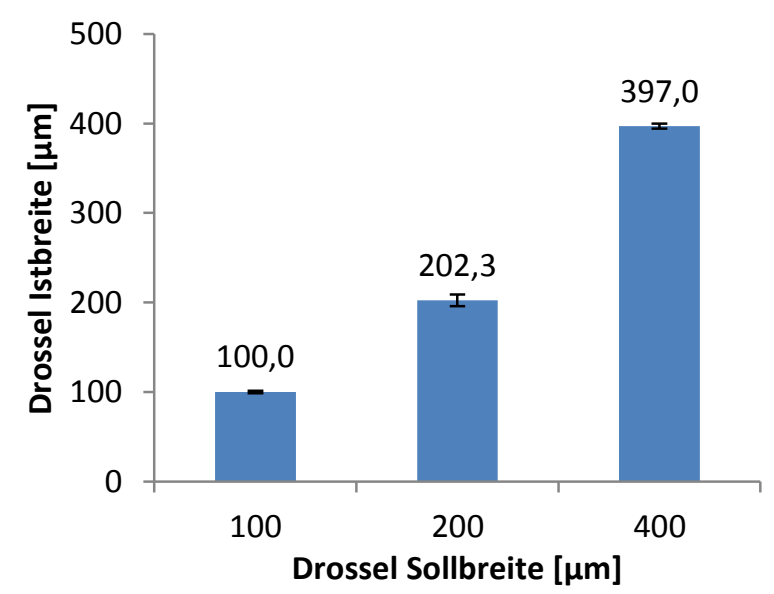

(d)

Abb. 70: Bewertung der Formtreue des Prozesses zur Herstellung individueller Tropfenerzeuger. (a) Abtragstiefen der Fluidkammern, (b) Düsenlängen, (c) Elektrodenbreiten auf dem piezoelektrischen Aktor und (d) Drosselbreiten. 
Abschließend werden in Abb. 71 die resultierenden Ergebnisse bzgl. der Drossellänge, der Fluidkammerbreite und des Durchmessers der Fluidzuführung gezeigt. Diese Größen sind während des gesamten Entwicklungsprozesses der vorliegenden Arbeit nicht verändert worden. Auch hier wird die geforderte Genauigkeit aus Kapitel 4 eingehalten. Der Mittelwert für die Breite der Fluidkammer errechnet sich dabei aus über 230 Messwerten, welche wiederum aus 23 Fertigungschargen über einen Zeitraum von 15 Monaten gewonnen worden sind.

Der vorgestellte Prozessflow wird mit der mikroelektronischen Fertigungstechnik von Mikrosystemen hinsichtlich Anschaffungskosten der Maschinen und notwendiger Prozesszeit verglichen. Der Fokus dieses Vergleiches liegt auf der Mikrostrukturierung des Siliziums, da die weiteren Fertigungsschritte in dem vorgestellten Prozessflow (Bonden, Strukturierung der Elektroden, Vereinzeln) weitestgehend standardisierter Fertigung entsprechen.

Für die Mikrostrukturierung werden vorliegend sowohl ein Lasersystem als auch eine Wafersäge eingesetzt. Die Anschaffungskosten (Laserquelle, Galvanometerscanner, motorbetriebene Achsen, Tisch und weitere Peripherie) für das UV Lasersystem Gator betragen ca. $260 \mathrm{~T} €$; für das IR Lasersystem LS 2000 / 8000 weniger, da eine kostenintensive Frequenzvervielfachung dieser Laserquelle nicht notwendig ist. Die Anschaffungskosten der Wafersäge DAD321 belaufen sich auf ca. $70 \mathrm{~T} €$ (Schneider, 2015). Insgesamt ergeben sich für vorliegenden Prozess der Mikrostrukturierung ein Anschaffungspreis der Maschinen von maximal 330 T€. Lawes (2007) und Schwesinger (2015) nennen für die Mikrostrukturierung mit mikroelektronischer Fertigungstechnik Anschaffungskosten von Trockenätzanlagen nach dem Bosch-Prozess (DRIE) in Höhe von mindestens $500 \mathrm{~T} €$, Mask Alignern von mindestens $200 \mathrm{~T} €$ sowie von Spin Coatern von mindestens $50 \mathrm{~T} €$. Trotz der hohen Anschaffungskosten hat sich das trockenchemische Ätzen aufgrund seiner hohen Reproduzierbarkeit und Materialvielfalt durchgesetzt (Glück, 2005; Hilleringmann, 2006), so dass die vorgestellte Mikrostrukturierungstechnik mit dem tiefen reaktiven Ionenätzen gegenübergestellt wird. Für Mikrostrukturierungsoperationen ist die Photolithographie grundlegender Bestandteil, so dass sich hierfür bereits Anschaffungskosten von mindestens $250 \mathrm{~T} €$ (Mask Aligner und Spin Coater) ergeben. In Verbindung mit den Maschinen für die Schichtätzung ergeben sich Maschinenkosten von mindestens $750 \mathrm{~T} €$. Hinzu kommen noch Anschaffungskosten für den Reinraum in Höhe von ca. $3 \mathrm{~T} € / \mathrm{m}^{2}$ (Tadigadapa und Najafi, 2003). Vergleicht man an dieser Stelle die Anschaffungskosten für den Maschinenpark für die Mikrostrukturierung, so wird festgehalten, dass die Kosten des vorliegenden Prozesses (Lasersystem und Wafersäge) über $50 \%$ günstiger gegenüber der standardisierten mikroelektronischen Fertigungstechnik sind. Die hohen Anschaffungskosten mikroelektronischer Fertigungstechnik relativieren sich nur bei

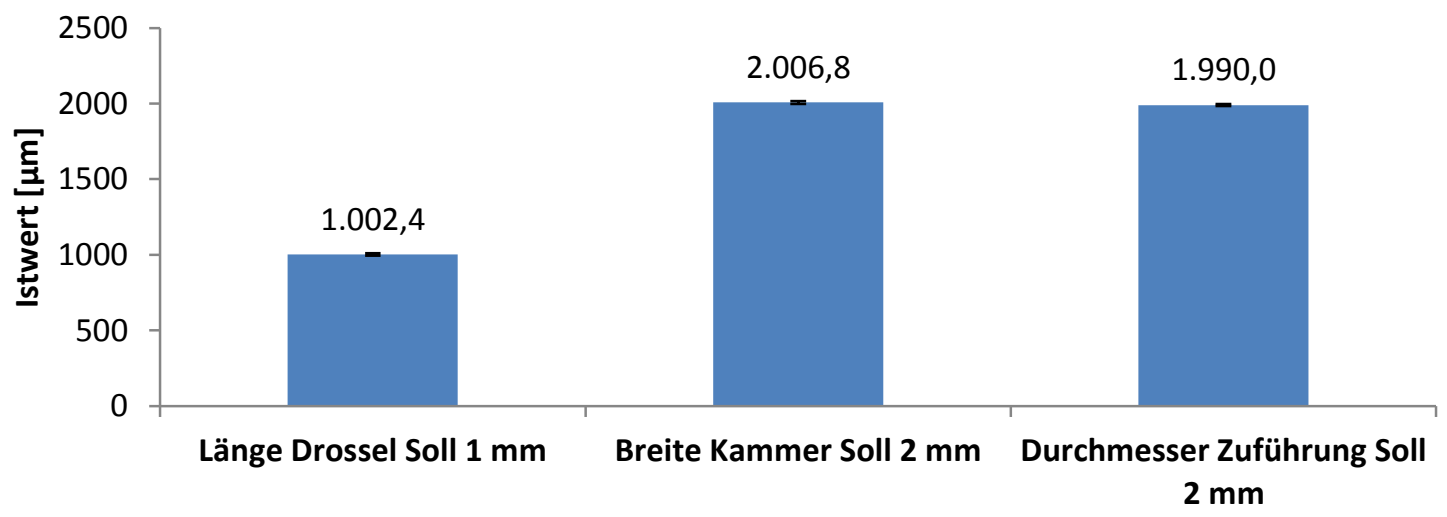

Abb. 71: Bewertung des Prozesses zur Herstellung individueller Tropfenerzeuger für die Größen Drossellänge, Fluidkammerbreite und Durchmesser der Fluidzufuhrbohrung. 
einer hohen Anzahl in Batchprozessen gleich gefertigter Mikrosysteme (Abgrall und Gué, 2007; Fujita, 2007; Ashida et al., 2010). Bei geringen Stückzahlen erfolgt keine Kostenreduktion. Zusätzlich ergeben sich für die Fertigung von Funktionsmodellen deutlich höhere Experimentierkosten, da für jedes Layout eine Maske für die Photolithographie erstellt werden muss sowie Chemikalien, wie bspw. Photoresist und Haftvermittler, notwendig sind.

Neben der Analyse der Anschaffungskosten gilt es auch die Prozesszeit für die Mikrostrukturierung gegenüberzustellen. Voraussetzung für die Schichtätzung stellt eine Maskierung auf dem Silizium dar. Im Idealfall ist strukturierter Photoresist ausreichend. Madou (2002) gibt für das trockenchemische Ätzen von Silizium mit einer Photoresistmaske eine Ätzrate von $5 \mu \mathrm{m} / \mathrm{min}$ bei einer Selektivität von 50:1 an. Höhere Ätzraten von bis $20 \mu \mathrm{m} / \mathrm{min}$ können erzielt werden, allerdings werden hierfür selektivere Maskierschichten, bspw. aus $\mathrm{SiO}_{2}$ oder $\mathrm{Au}$, benötigt. Zieht man für den Vergleich einen Druckchip mit $100 \mu \mathrm{m}$ tief abladierten Strukturen heran, so wird eine reine Ätzzeit von 20 Minuten benötigt. Unterschiedlich breite und tiefe Mikrostrukturen können in gewissem Maße über die Dimensionierung der Maskierung erzeugt werden. Schwesinger, Dehne und Adler (2009) beschreiben hierzu die Ätzverzögerung „Etch Lag“, d. h. die Abhängigkeit der Ätzrate von der Strukturbreite der Trenches. Hinzu kommt noch die Fluidzufuhrbohrung, welche nach dem Ätzen der fluidmechanischen Strukturen von der Rückseite des Siliziums vorgenommen wird. Das Silizium besitzt eine Dicke von $525 \mu \mathrm{m}$, wovon bereits $100 \mu \mathrm{m}$ abgetragen sind. Es bleibt eine Restdicke von $425 \mu \mathrm{m}$ übrig, wofür 85 Minuten benötigt werden. Insgesamt ergibt sich eine reine Ätzzeit von 105 Minuten. Hinzu kommt noch die Prozesszeit für die zweimalige Strukturierung der Photoresistschicht, welche sich aus Temperung, Belichtung und Entwicklung zusammensetzt. Clariant (1997) gibt bspw. für den Photoresist $A Z^{\circledR} 9200$ für das trockenchemische Ätzen eine Prozesszeit von je zwei Minuten an. Es ergibt sich somit in Summe eine Prozesszeit von 109 Minuten. Für den vorgestellten Prozessflow aus Lasersystem und Wafersäge ergibt sich eine reine Prozesszeit von 65 Minuten für die Mikrostrukturierung von fünf Druckchips (100 $\mu \mathrm{m}$ tiefe Ablation und $100 \mu \mathrm{m}$ breite Düsen). Dies zeigt deutlich, dass der vorgestellte Prozess für geringe Stückzahlen deutlich kürzer ist als die standardisierte Fertigungstechnik und ideal für die Erstellung von Funktionsmodellen für die Mikrosystemtechnik ist. Erst bei mehr als acht Druckchips ist die mikroelektronische Fertigung effizienter. An dieser Stelle muss allerdings erwähnt werden, dass vorliegend nur die reinen Prozesszeiten betrachtet werden. Werden noch Fertigungs- und Lieferzeiten von Masken für die Photolithographie berücksichtigt, wird deutlich, dass vorgestellter Prozess eine höhere Experimentierhandhabbarkeit bietet. Kürzere Prozesszeiten werden mit trockenchemischen Ätzen durch Erhöhung der Ätzrate erzielt. Allerdings ergibt sich hierdurch der Sachverhalt, dass neben Photoresist auch Hartmasken, wie bspw. aus $\mathrm{SiO}_{2}$, aufgebracht und strukturiert notwendig sind.

Darüber hinaus ist der Schulungsaufwand der Operatoren geringer, da vorliegend für die Mikrostrukturierung ein Lasersystem und eine Wafersäge ausreichend sind. Mit mikroelektronischer Fertigung sind mindestens die Bedienung von Spin Coater, Mask Aligner, Ätzanlage und Heizquelle notwendig.

Zusammenfassend wird an dieser Stelle festgehalten, dass die gestellte Aufgabe in Kapitel 4, individuelle Funktionsmodelle eines Mikrosystems, vorliegend validiert an einem Mikrotropfenerzeuger, in weniger als einem Tag zu fertigen, erfüllt wurde. In weniger als 10 Stunden werden fünf identische Tropfenerzeuger für Untersuchungen bereitgestellt, und damit der Entwicklungszyklus eines Produktes verkürzt. Innerhalb eines Tages besteht somit die Möglichkeit, ein Redesign eines Druckkopfmusters durchzuführen. Mit 15 Düsen in Summe steht eine ausreichende Anzahl an Versuchsträgern bereit. 


\subsection{Inbetriebnahme der Mikrotropfenerzeuger}

Nach der Herstellung der einzelnen Druckchips müssen diese in Betrieb genommen werden. Die Düsenplatte vorliegenden Druckkopfes besteht aus einkristallinem Silizium. Darüber befindet sich die $100 \mu \mathrm{m}$ dicke Borosilikatglasmembran. Voraussetzung für einen stabilen und reproduzierbaren Tropfenbildungsprozess ist, dass eine Benetzung der Düsenplatte mit Fluid vermieden wird. Denn bildet sich ein Fluidfilm über den Düsenauslass, besteht die Gefahr, dass das beschleunigte Fluid im Düsenkanal diese Barriere nicht durchbrechen kann und kein Tropfenabriss stattfindet. Reines Silizium stellt für die Benetzung mit Wasser eine ideale hydrophobe ( $\triangleq$ wasserabstoßende) Oberfläche dar. Im Laufe der Lagerung bildet sich auf dem Silizium allerdings natives Oxid, welches den Kontaktwinkel zwischen einem abgesetzten Wassertropfen auf dem Substrat reduziert und damit eine hydrophile ( $\hat{=}$ wasserliebende) Oberfläche bietet. Franssila (2010) gibt für natives Oxid einen Kontaktwinkel von $45^{\circ}$ an. Das darüber liegende Borosilikatglas bildet ebenfalls mit Wasser eine hydrophile Oberfläche mit einem Kontaktwinkel von $\approx 32^{\circ}$ (Sumner et al., 2004). Beide Oberflächen verhalten sich insbesondere gegenüber Wasser hydrophil. Daher ist der erste Schritt bei der Inbetriebnahme der Druckchips, die Düsenplatte in ihrer Oberfläche so zu modifizieren, dass eine verringerte Haftung des Fluides an ihr entsteht. Dies bedeutet den Kontaktwinkel zu erhöhen.

Nach erfolgreicher Oberflächenmodifikation der Düsenplatte werden die Druckchips sowohl fluidisch als auch elektrisch kontaktiert. Ziel ist es, dass die Inbetriebnahme der einzelnen Mikrotropfenerzeuger rasch und intuitiv durchführbar ist, um eine hohe Experimentierhandhabbarkeit zu gewährleisten. Darüber hinaus wird eine Minimierung des Risikos, einzelne Druckchips aufgrund ihrer dünnen, spröden und fragilen Komponenten zu beschädigen, gefordert. Dies wird dadurch erreicht, dass die Mikrotropfenerzeuger während der Inbetriebnahme keiner Montagekraft ausgesetzt werden dürfen.

Aus den soeben beschriebenen Forderungen folgt eine Funktionsseparation für die Inbetriebnahme. Nicht der Druckchip selbst, sondern ein Verbindungselement wird für die elektrische und fluidische Versorgung genutzt (siehe Abb. 72). Die Druckchips werden nach ihrer Herstellung und der Oberflächenmodifikation der Düsenplatte auf einen Zwischenträger montiert. Hier erfolgt deren elektrische Kontaktierung über Lötverbindungen zu den integrierten Leiterbahnen. Von Bedeutung hierbei ist, dass die Elektroden auf dem piezoelektrischen Wandler

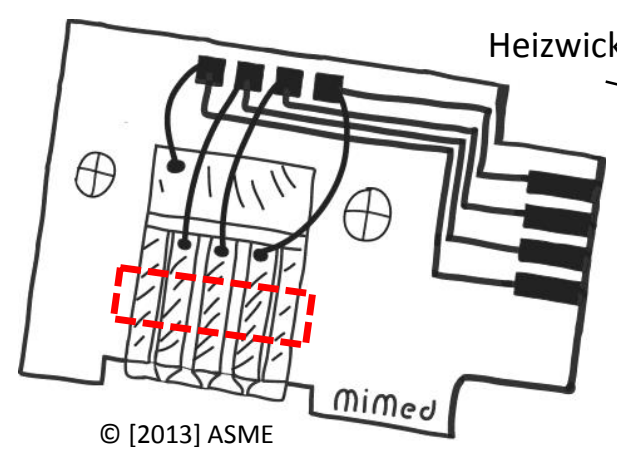

(a)

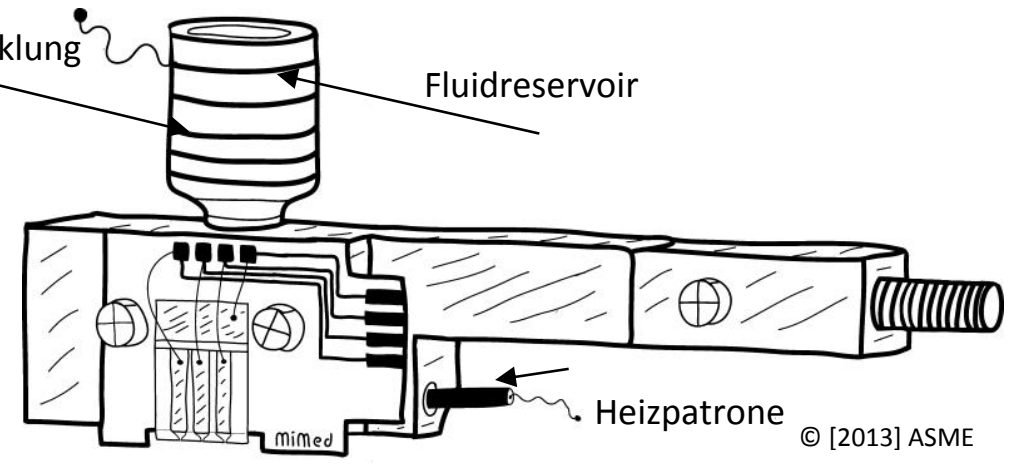

(b)

Abb. 72: Schematische Darstellung der Funktionsseparation für die Inbetriebnahme der Druckchips. (a) Tropfenerzeuger wird auf ein stabiles Zwischenstück montiert und elektrisch kontaktiert. Die Mitte der Elektroden ist frei einsehbar, so dass Hubmessungen mittels Laserinterferometrie ermöglicht werden. (b) Zwischenstück an der Aufnahmevorrichtung. Zusätzliche Bestandteile sind ein Fluidreservoir, Heizelemente und ein seitlicher Gewindebolzen für den Einbau in einen Prüfstand. Entnommen aus Kagerer et al. (2013c). 
in ihrer Mitte frei einsehbar sind, um im weiteren Verlauf der Untersuchungen auch Hubmessungen mittels Laserinterferometrie durchzuführen. Die Hubmessungen dienen als Funktionsprüfung der Aktorik. Es gilt hierbei, einen über den Projektzeitraum gleichbleibenden Energieeintrag in Form der Biegung des bimorphen Aktors den Mikrotropfenerzeugern zuzuführen. Der Zwischenträger wird im Anschluss an eine Aufnahmevorrichtung montiert. Es muss ein ausreichend steifes Material gewählt werden, um den äußeren Kräften während der Befestigung an der Aufnahmevorrichtung zu widerstehen. Die Verbindung zwischen Zwischenträger und Aufnahmevorrichtung ist dicht auszuführen, so dass kein Fluid an dieser Stelle entweicht. Um auch Anwendungen im 3D Druck zu ermöglichen, werden Heizelemente der Dosiervorrichtung hinzugefügt, um phasenübergreifende Medien tropfenweise abzugeben. Hierzu wird ein Feststoff für seine Dosierung mittels thermischer Energie in den flüssigen Zustand überführt. Bei Auftreffen der Tropfen auf einem Substrat erfolgt deren Rücktransformation in den festen Zustand. Als Heizelemente werden eine Heizpatrone unterhalb des Druckchips und eine Heizwicklung um das Fluidreservoir eingesetzt. Die Heizwicklung schmilzt den Feststoff im Fluidreservoir, während die Heizpatrone den Druckchip selbst heizt. Somit ist eine homogene Temperaturverteilung im System sichergestellt. Zusätzlich wird der Einbau des kompletten Dosiersystems in Prüfständen für die Tropfenauswertung durch nötige Vorrichtungen ermöglicht.

\subsubsection{Oberflächenmodifikation der Düsenplatte}

Bei der Tropfenerzeugung mit dem vorliegenden Druckkopf kann es zur Entstehung eines Flüssigkeitsfilmes auf der Düsenplatte kommen. Dieser Flüssigkeitsfilm bremst bzw. lenkt ausgestoßene Tropfen ab und kann damit eine stabile und reproduzierbare Tropfenentstehung verhindern.

Damit eine verminderte Haftung des Fluides an der Düsenplatte erfolgt, wird ihre Oberfläche so modifiziert, dass diese einen größeren Kontaktwinkel $\theta_{\text {Kont }}$ mit dem Fluid bildet. Hierzu wird ein Tropfen einer Flüssigkeit (aufgrund der hohen Oberflächenspannung von $\approx 72 \mathrm{mN} / \mathrm{m}$ Wasser) auf ein Substrat abgesetzt und der resultierende Winkel an der Kontaktstelle zwischen Fluid und Oberfläche gemessen. Je höher dieser ist, desto höher ist der Grad der Hydrophobizität. Die Düsenplatte vorliegenden Mikrotropfenerzeugers besteht aus Silizium und der Borosilikatglasmembran. Auf reinem Silizium bildet Wasser wie bereits oben beschrieben einen hohen Kontaktwinkel aus, jedoch entsteht mit der Zeit auf Siliziumoberflächen ein natives Oxid, welches hydrophil ist und den Kontaktwinkel verringert. Das Borosilikatglas verhält sich von Natur aus immer hydrophil.

Um den Kontaktwinkel durch den Auftrag einer Schicht zu erhöhen, stehen verschiedene Verfahren, wie z. B. das Vakuumbedampfen, die chemische oder auch die physikalische Gasphasenabscheidung, zur Verfügung. Ein für die Oberflächenmodifikation besonders geeignetes Material ist Polytetrafluorethylen (PTFE, Teflon ${ }^{\circledR}$ ). Hiermit werden superhydrophobe Oberflächen erreicht. Die Abscheidung von PTFE wird meist mit einem CVD Prozess oder durch einen physikalischen HF Sputterprozess (High Frequency) erreicht. Leider sind die dafür notwendigen Gerätschaften am Lehrstuhl nicht vorhanden. Zur Abscheidung von dünnen Schichten steht eine DC Sputteranlage (Direct Current) von JEOL (Germany) Modell JFC1200 FINE COATER zur Verfügung, welche zur Vergoldung von Objekten für das Rasterelektronenmikroskop genutzt wird. Allerdings besteht damit nur die Möglichkeit, elektrisch leitfähige Targets einzusetzen, weshalb ein modifiziertes PTFE Target verwendet wird. Dieses ist zur Reduzierung des elektrischen Widerstandes zu einem Anteil von $25 \%$ mit Kohle 
gefüllt. Der Oberflächenwiderstand reduziert sich hierdurch von $10^{17}$ für ungefülltes auf $10^{2} \Omega$ für gefülltes PTFE (ElringKlinger Kunststofftechnik, 2014).

Für die Ermittlung effizienter Prozessparameter für die PTFE Beschichtung werden die beiden veränderbaren Parameter der DC Sputteranlage, der Strom der Plasmaanregung und der Prozesskammerdruck variiert und anschließend die Kontaktwinkel mit einem liegenden Auflichtmikroskop gemessen. Der Abstand von Substratoberfläche und Target wird auf ca. 60 mm eingestellt; geringere Abstände führen zu höherer Beanspruchung der Oberfläche. Die Prozesszeit für die Versuche beträgt konstant 10 Minuten, um den Rapid Manufacturing Grundgedanken aufrecht zu erhalten. Als Prozessgas dient Argon. Das gefüllte PTFE wird auf $1 \mathrm{~mm}$ dicke Glasobjektträger gesputtert. Für das Absetzen eines Wassertropfens wird eine Mikrospritze der Firma VWR International verwendet. Es werden Tropfen mit $1 \mu 1$ Volumen abgesetzt.

Abb. 73 zeigt die erzielten Ergebnisse. Es stellt sich ein Maximum des Kontaktwinkels bei einem Druck von $6 \mathrm{~Pa}$ ein. Dieser ist weitestgehend unabhängig von dem Sputterstrom. Bei geringerem Vakuum, d. h. höherem Druck, sinkt der Kontaktwinkel. Der Grund liegt in der Reduzierung der mittleren freien Weglänge der ausgeschlagenen Teflonteilchen. Liegt dagegen der Druck in der Prozesskammer unter $6 \mathrm{~Pa}$, ist zu wenig Argon vorhanden, um ein dichtes Plasma zu ermöglichen. Auch ein geringfügiger Einfluss des Stroms auf das Ergebnis ist erkennbar. In dem Arbeitsbereich zwischen 4 und $10 \mathrm{~Pa}$ wird durch einen kleineren Strom ein höherer Kontaktwinkel erzielt. Die Untersuchungen zeigen zudem, dass hohe Stromstärken und das daraus resultierende starke Plasma oft zu „Einbranderscheinungen“ auf dem Substrat führen. Daher werden als Parameter für die Oberflächenmodifikation der Düsenplatte ein Strom von $10 \mathrm{~mA}$ und ein Arbeitsdruck von $6 \mathrm{~Pa}$ verwendet. Abb. 74 zeigt abgesetzte Wassertropfen auf PTFE behandelten Substraten für verschiedene Werte des Prozesskammerdruckes.

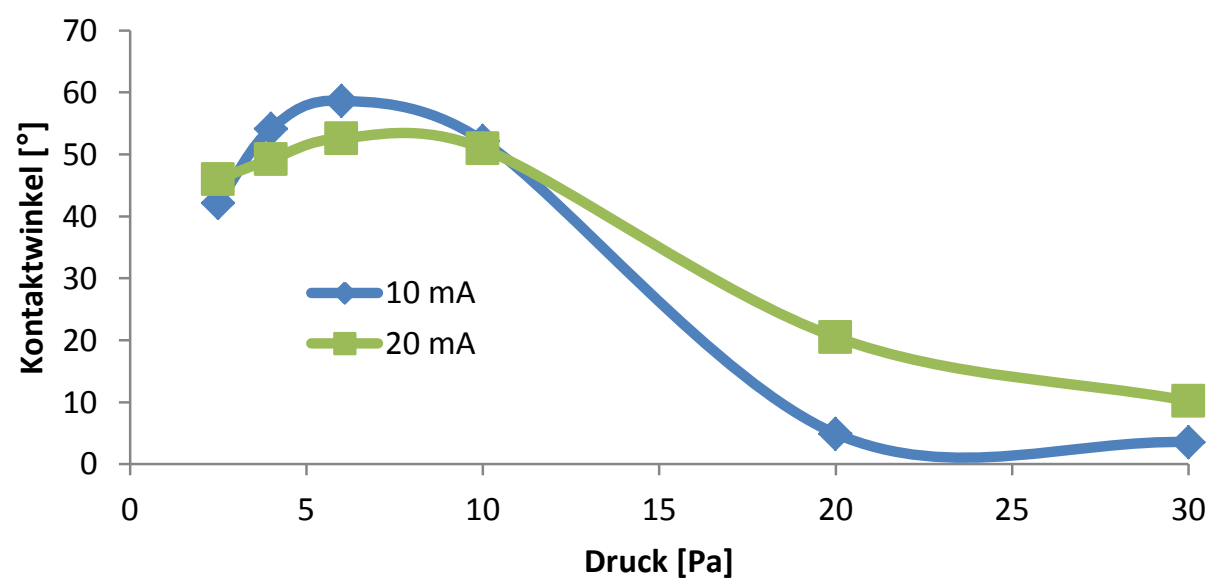

Abb. 73: Kontaktwinkel in Abhängigkeit des Arbeitsdruckes und der Höhe des Betriebsstromes.

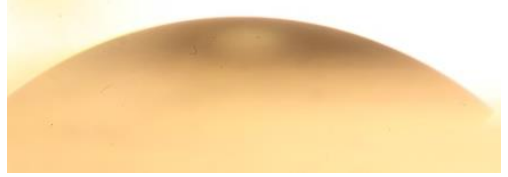

(a)

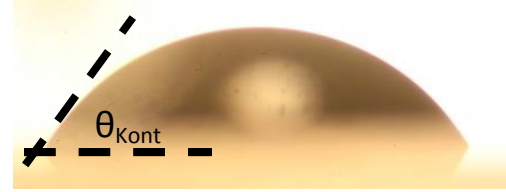

(b)

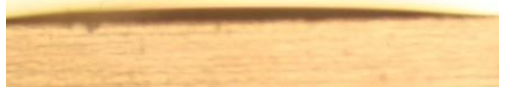

(c)

Abb. 74: Abgesetzter Wassertropfen auf PTFE behandelte Glasobjektträger bei einem Strom von $10 \mathrm{~mA}$ und einem Prozesskammerdruck von (a) 2,5 Pa, (b) $6 \mathrm{~Pa}$ und (c) $20 \mathrm{~Pa}$. 
Die Druckchips selbst werden für den Prozess in ein Kupferband eingewickelt, um den piezoelektrischen Wandler nicht direkt den in dem Plasma wirksamen elektrischen Feldern auszusetzen. Nur die Düsenplatte bleibt exponiert. Auch wenn bei erzielten Kontaktwinkeln von $\approx 60^{\circ}$ noch nicht von hydrophoben Oberflächen gesprochen wird, so kann dennoch festgehalten werden, dass aufgrund des größeren Kontaktwinkels für die Handhabbarkeit im Versuchsbetrieb eine deutliche Verbesserung gegenüber unbehandelten Düsenplatten erreicht wird.

\subsubsection{Montage der Druckchips auf eine Platine}

Bezugnehmend auf die Anforderungen der Inbetriebnahme wird ein Zwischenträger benötigt, auf dem die Mikrotropfenerzeuger montiert sind. Abb. 75 zeigt, dass das Zwischenstück eine Platine darstellt, auf die die Mikrotropfenerzeuger mit dem Zweikomponenten Epoxidharzklebstoff UHU PLUS ENDFEST 300 der Firma UHU geklebt werden. Über eine Bohrung in der Platine mit einem Durchmesser von $2 \mathrm{~mm}$ wird die Fluidzufuhr von der Rückseite des Siliziumchips auf die Rückseite der Platine verlegt. Der Klebstoff wird zusätzlich um den Mikrotropfenerzeuger verteilt, so dass kein Fluid über die Zuführung und die Auflagefläche seitlich entweicht. Für das Absetzen der Druckchips auf die mit Klebstoff bestrichene Auflagefläche stehen Justagelinien zur Verfügung. Diese erlauben die konzentrische Ausrichtung der beiden Fluidzufuhrbohrungen.

Das Kernmaterial der Platine ist 1,5 mm dickes Aluminium. Darüber befindet sich eine 0,1 mm starke Epoxyschicht als elektrische Isolation gefolgt von einer verzinnten 0,07 mm dicken Kupferschicht der Leiterbahnen und der Lötpads. Abschluss des Schichtaufbaus stellt $0,1 \mathrm{~mm}$ dicker Lötstopplack dar.

Die elektrische Kontaktierung des Druckchips erfolgt über Lötverbindungen zu den Anschlusspads. Insgesamt stehen sechs Pads mit einer Fläche von 2 x $2 \mathrm{~mm}^{2}$ zur Verfügung. Das Setzen der Lötpunkte auf den Elektroden erfolgt in ihrem vorderen Teil. So ist der Großteil ihrer Fläche frei einsehbar und die Fokussierung eines Laserstrahls für interferometrische Hubmessungen wird während der Tropfenerzeugung oder auch zur Kontrolle des Aktorverhaltens ermöglicht. Der Laserspot wird frei auf die Mitte der Elektroden fokussiert, da basierend auf der Balkentheorie die höchsten Hübe an dieser Position auftreten. Auch wenn der derzeitige Aufbau des Tropfenerzeugers drei Düsen enthält, so besteht in Zukunft die Möglichkeit, bis zu fünf Düsen zu integrieren und somit die Integrationsdichte zu erhöhen. Die korrespondierenden Leiterbahnen mit einer Breite von $0,4 \mathrm{~mm}$ führen $\mathrm{zu}$ einer zentralen elektrischen Anschlussstelle, an der die notwendige Energie für den Tropfenausstoß über einen Platinenstecker dem Druckchip zugeführt wird.

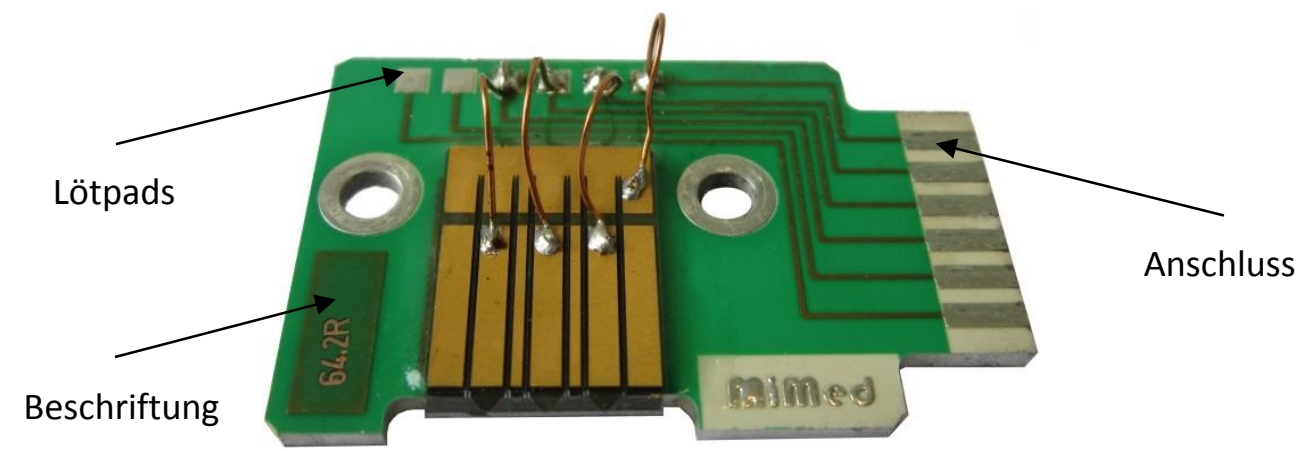

Abb. 75: Zwischenträger für den Druckchip auf Platinenbasis. Die elektrischen Kontaktierungen erfolgen über Lötverbindungen zu den Lötpads mit angebundenen Leiterbahnen, die zu einer zentralen Anschlussmöglichkeit für einen Platinenstecker führen. Zusätzlich enthält die Platine ein mögliches Beschriftungsfeld zur Kennzeichnung der Druckchips. 
Die Handhabbarkeit der Druckchips wird durch die Verwendung der Platine erleichtert. Zusätzlich bietet Aluminium mehr Stabilität und eine höhere Wärmeleitfähigkeit gegenüber standardisierten Epoxidharzkernen für Platinen, so dass die generierte Wärme von der Aufnahmevorrichtung über die Platine direkt dem Druckchip zugeführt wird. Die Platine bietet ausreichend Platz, um die Mikrotropfenerzeuger zu beschriften. Die Düsenplatte ist für mikroskopische Aufnahmen durch eine Aussparung einsehbar.

Die Platine wird mit zwei M3 Schrauben an der Aufnahmevorrichtung montiert. Hierfür stehen zwei Bohrungen zur Verfügung. So ist der Austausch einfach und schnell durchführbar. Es werden ca. 30 Minuten pro Mikrotropfenerzeuger für die Klebung des Druckchips auf die Platine (ausgenommen Aushärtezeit des Klebstoffes), deren elektrische Kontaktierung mittels Lötverbindungen und der Beschriftung der Platine benötigt. Die Platine nimmt eine Fläche von $41 \times 26 \mathrm{~mm}^{2}$ ein und wird von der Firma LeitOn zugekauft. Die Kosten belaufen sich auf $2,20 € /$ Platine.

\subsubsection{Aufnahmevorrichtung für platinenbasierte Druckchips}

Nach der Montage der Druckchips auf dem platinenbasierten Zwischenträger wird dieser an die Aufnahmevorrichtung montiert. Die Aufnahmevorrichtung beinhaltet Möglichkeiten für die Fluidzuführung, das Beheizen des Dosiersystems und dessen Einbau in einen Tropfenbeobachtungsstand. Abb. 76 zeigt die Aufnahmevorrichtung als Konstruktionsmodell im Querschnitt. Die wesentlichen Komponenten sind ein Grundkörper zur Aufnahme der platinenbasierten Druckchips, ein Fluidreservoir, zwei Heizelemente, ein Montageadapter für den Einbau in einen Tropfenbeobachtungsstand und ein Isolationsblock aus PTFE. Die Aufnahmevorrichtung ist modular aufgebaut, so dass jederzeit ein Austausch einzelner Komponenten, wie bspw. der Einbau eines größeren Fluidreservoirs, möglich ist.

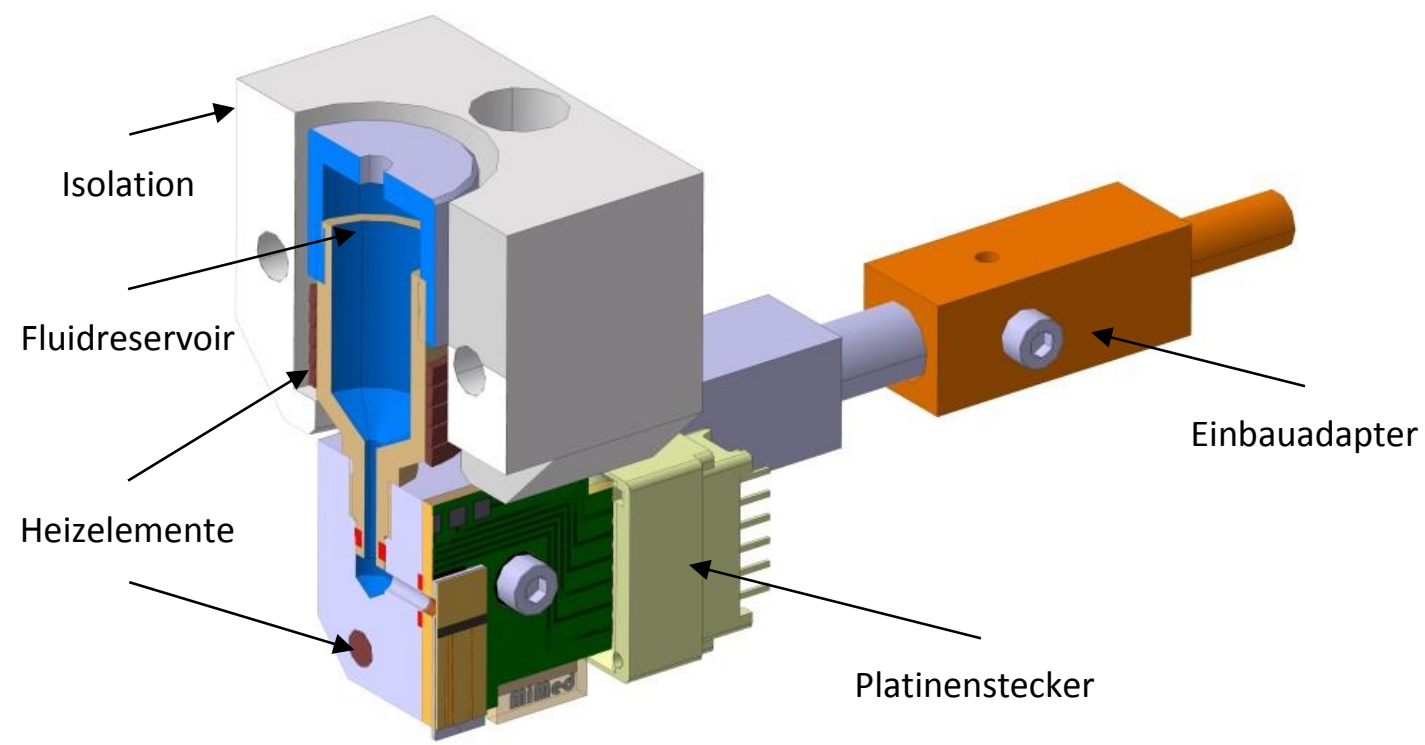

Abb. 76: CAD Konstruktionsmodell im Querschnitt von der Aufnahmevorrichtung mit Platine inkl. Mikrotropfenerzeuger. $\mathrm{Zu}$ erkennen sind Heizelemente sowohl um das Fluidreservoir als auch unterhalb des Mikrotropfenerzeugers, der PTFE Isolationsblock und der Adapter für den Einbau in einen Tropfenbeobachtungsstand. Der vordere Teil der Aufnahmevorrichtung (Grundkörper) wird über einen Stahlbolzen in den Adapter geschoben und über eine Madenschraube bei definierter Ausrichtung fixiert. Das Fluid wird über einen Kanal aus dem Fluidreservoir zu dem Mikrotropfenerzeuger geleitet. Über einen Platinenstecker wird der Mikrotropfenerzeuger von außen elektrisch versorgt. 
Sowohl der Grundkörper als auch das Fluidreservoir sind aus Aluminium gefertigt. Aluminium besitzt mit $236 \mathrm{~W} / \mathrm{m} \cdot \mathrm{K}$ eine ausreichend hohe Wärmeleitfähigkeit, um die generierte Wärme von den Heizelementen sowohl in das Fluidreservoir als auch zu dem Druckchip zu leiten. Über einen Kanal im Grundkörper mündet der Auslass des Fluidreservoirs in die Fluidzuführung der Platine. Das Fluidreservoir ist zylinderförmig aufgebaut und wird über einen Aluminiumdeckel luftdicht verschlossen. Das Fluidreservoir besitzt ein ausreichendes Aufnahmevolumen von ca. $2 \mathrm{~cm}^{3}$. Die Abdichtung von Fluidreservoir zu Grundkörper und von Grundkörper zu Platine erfolgt mit O-Ringen aus Perlast ${ }^{\circledR}$ G80A, einem Perfluorelastomer von C. Otto Gehrckens. Perlast ${ }^{\circledR}$ G80A besitzt sowohl eine hohe thermische als auch chemische Beständigkeit, so dass selbst aggressive Medien unter erhöhter Temperatur dosiert werden können. Das Fluidreservoir wird über eine Schraubverbindung an dem Grundkörper befestigt. Sein Austausch gestaltet sich hierdurch einfach. Um auch das Dosieren von phasenübergreifenden Medien zu erlauben, werden Heizelemente der Aufnahmevorrichtung hinzugefügt. Es werden eine Heizwicklung um das Fluidreservoir und eine Heizpatrone direkt unterhalb der Platine platziert. Die Wicklung hat eine Heizleistung von $125 \mathrm{~W}$; die Patrone besitzt eine Heizleistung von $70 \mathrm{~W}$. Beide Elemente stammen von der Firma Türk+Hillinger und ermöglichen eine homogene Temperaturverteilung im System. Die Curietemperatur des verwendeten piezoelektrischen Grundmaterials VIBRIT 1876 von Johnson Matthey Catalysts (Germany) beträgt $121^{\circ} \mathrm{C}$. Eine höhere Temperatur würde zu der Depolarisation des Materials führen, womit beide Heizelemente ausreichend dimensioniert sind. Um dennoch eine Wärmeabgabe an die Umgebung und die Gefahr vor Verletzungen an der Heizwicklung zu verringern, wird um das Fluidreservoir ein PTFE Körper als thermische Isolation hinzugefügt. PTFE verfügt mit einem Wärmeleitwert von ca. $0,25 \mathrm{~W} / \mathrm{m} \cdot \mathrm{K}$ über eine ausreichend thermisch dämmende Eigenschaft. Zwei Leitungsfühler der Firma Thermo Sensor messen die Temperatur und leiten diese an zwei externe Regler. Abb. 77 zeigt die realisierte Aufnahmevorrichtung.

Um die Tropfenbildung zu analysieren und zu bewerten, ist es erforderlich, dass die Düsenplatte senkrecht von einer Kamera erfasst wird. Hierzu gilt es, den Grundkörper inklusive des platinenbasierten Druckchips entsprechend auszurichten. Der Grundkörper besitzt an seinem Ende einen Stahlbolzen, über den der Grundkörper in einem Montageadapter befestigt und die korrekte Position mit der Madenschraube fixiert wird. Über ein seitliches Gewinde besteht die Möglichkeit, das komplette Dosiersystem in einen Messstand einzubauen.

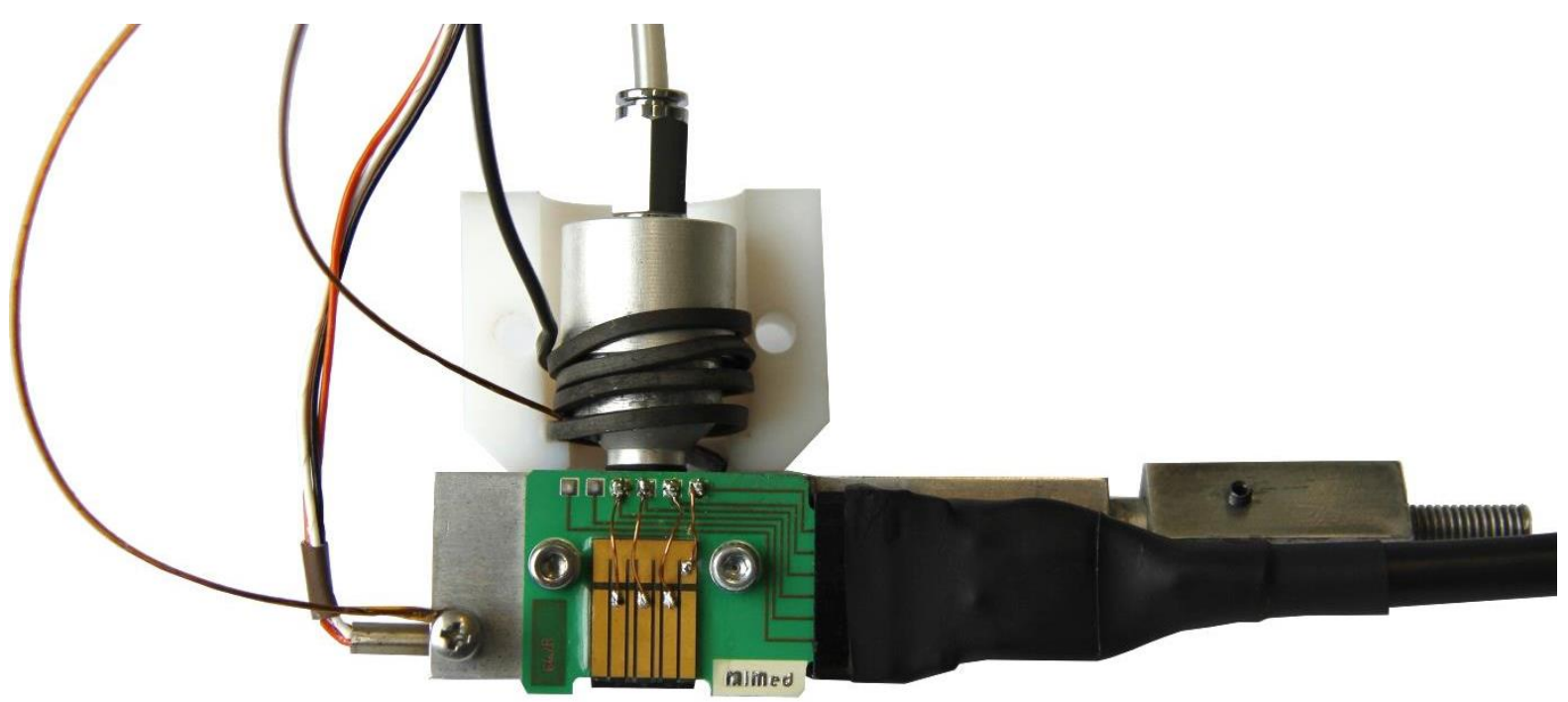

Abb. 77: Realisierte Aufnahmevorrichtung mit montierter Platine inkl. Mikrotropfenerzeuger. Gegenüber Abb. 76 sind die beiden Leitungsfühler für die Temperaturregelung hinzugefügt. 
Die Platine wird mit zwei Schrauben an dem Grundkörper befestigt. Da das Kernmaterial der Platine 1,5 mm dickes Aluminium ist, widersteht das Material hohen Anzugskräften, so dass im Ergebnis eine dichte Verbindung von Grundkörper zu Platine geschaffen ist. Die elektrische Versorgung der Platine wird über einen handelsüblichen Platinenstecker realisiert. Die Länge der gesamten Aufnahmevorrichtung beträgt ca. $110 \mathrm{~mm}$. Sie bietet eine gute Handhabbarkeit bei der Durchführung von Experimenten. Der Wechsel der platinenbasierten Druckchips ist in weniger als einer Minute möglich.

Für die Druckversorgung des gesamten Dosiersystems wird ein Kunststoff Druckluftschlauch über eine Schnellkupplung der Firma Festo an den Deckel des Fluidreservoirs angeschlossen. An dem Ende des Schlauches ist eine luftgefüllte Spritze befestigt. Ein T-Stück in diesem Schlauch leitet zusätzlich den resultierenden Druck, der auch auf das Fluid im Reservoir einwirkt, auf den Differenzdrucksensor DS2-010 (Messbereich: \pm 1250 Pa) der Firma Kalinsky Sensor Elektronik. Durch Anlegen eines Überdrucks verteilt sich das Fluid gleichmäßig in der Pumpkammer und eventuell vorhandene energieabsorbierende Luftblasen werden aus dem System gespült. Nach erfolgtem Befüllen ist ein Unterdruck anzulegen, um ein Austropfen des Fluides aus den Düsen zu vermeiden. In einem externen Laborgehäuse sind sowohl eine Druckanzeige DA-010 der Firma Kalinsky Sensor Elektronik als auch zwei Temperaturregler mit PID (proportional-integral-derivative) Verhalten UR3274 der Firma Wachendorff Prozesstechnik integriert. Abb. 78 zeigt das externe Laborgehäuse und eine Wärmebildkameraaufnahme des Dosiersystems. Die Temperaturverteilung innerhalb des Systems ist homogen.

Anstatt des Fluidreservoirs besteht auch die Möglichkeit, weitere Arten von Reservoiren über die Gewindebohrung an den Grundkörper anzubringen. Sofern keine Temperatur gefordert ist, wird eine offene Spritze als Fluidreservoir verwendet. Die korrekte Einstellung des Unterdruckes erfolgt hierbei über die horizontale Positionierung des Füllstandes in der Spritze zum Düsenauslass. Dabei entspricht eine Höhendifferenz von einem Millimeter einem Druck von 9,8 Pa.

\subsubsection{Kontrolle von Düsenfunktionalität und Hub des bimorphen Aufbaus}

Bevor fluidmechanische Untersuchungen mit dem Mikrotropfenerzeuger durchgeführt werden, sind die Düsenfunktionalität und auch die Hübe des Membranaktors zu prüfen. Wie bereits beschrieben, dehnt sich der Aktor in die darunterliegende Fluidkammer, sobald ein elektrischer Puls an die korrespondierende Elektrodenzunge angelegt wird. Die Biegung des

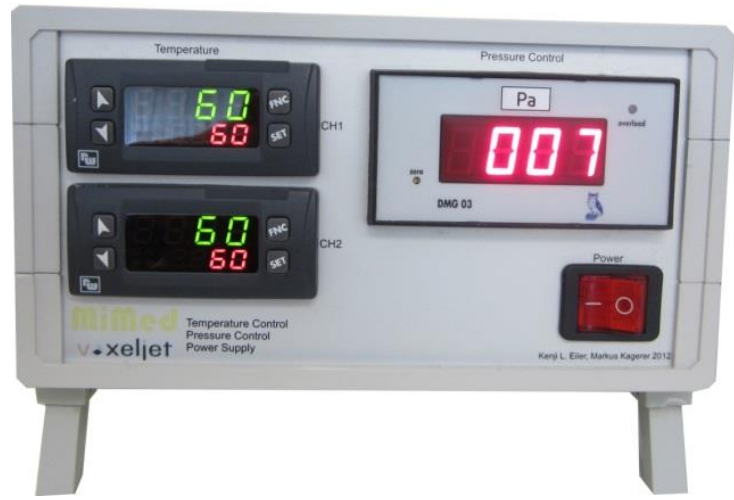

(a)

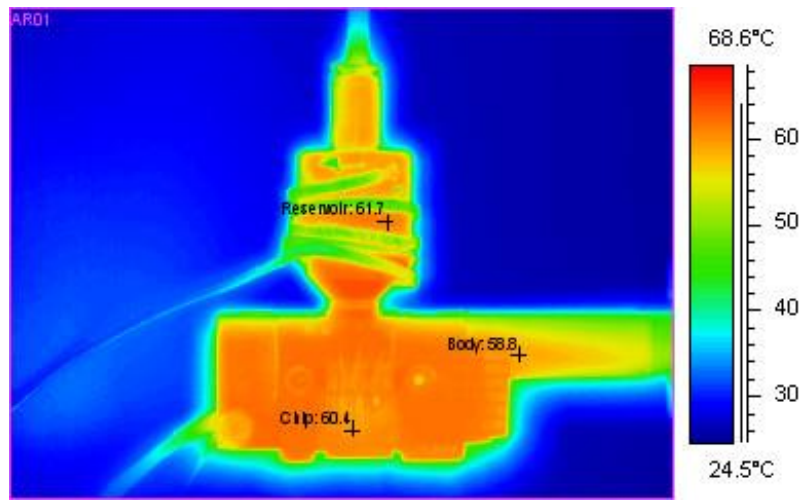

(b)

Abb. 78: (a) Externes Laborgehäuse mit Temperaturreglern und Druckanzeige und (b) Wärmebildaufnahme des beheizten Dosiersystems bei $60^{\circ} \mathrm{C}$. Eine homogene Temperaturverteilung im System ist erkennbar (Kamera: ThermaCAM SC 500 von FLIR SYSTEMS). 
Aktors ruft dabei eine Volumenverringerung der fluidgefüllten Kammer hervor, wodurch der benötigte Druckimpuls für den Tropfenausstoß initiiert wird. Je größer diese Biegung ist, desto größer ist der Druckimpuls. Da für die Erhebung aussagekräftiger Daten während fluidmechanischer Untersuchungen ein gleich hoher Energieeintrag gefordert ist, werden die Hübe des Aktors messtechnisch überprüft. Fehler in der Aktorik sind bspw. eingeschlossene Luftblasen in der Klebschicht oder lockere Lötkontakte. Darüber hinaus gilt es, auch fehlerhafte Düsen aus den Experimentreihen auszuschließen, da die Düsen eventuell während des Vereinzelungsprozesses der Druckchips aus der Waferebene heraus durch Partikel verstopft sind (siehe Abb. 79 (a)).

Die Düsenprüfung erfolgt vor der Montage der Druckchips auf die Platine, um möglichen Ausschuss frühzeitig während des Inbetriebnahmeprozesses zu erkennen und somit weitere Kosten in Form der Platine zu vermeiden. Für die Prüfung werden die Druckchips in eine formschlüssige Nut in eine Spülvorrichtung gelegt und manuell fixiert. Mit einer luftdichten Spritzflasche wird destilliertes Wasser der Vorrichtung zugeführt und über einen Kanal in den Druckchip, und somit zur Düsenspülung, geleitet. Abb. 79 (b) zeigt die Ergebnisse von drei unterschiedlich breiten Düsen mit einer Kantenlänge von 50, 70 und $100 \mu \mathrm{m}$. Die höchste Ausfallrate wird bei der breitesten Düse mit $100 \mu \mathrm{m}$ Kantenlänge erzielt. Es ist ersichtlich, dass sich hier Partikel leichter während der Lagerung, einem Transport oder generell auch während des Prozesses absetzen können. Dennoch stellt das Ergebnis einen sehr guten Wert dar, da man an dieser Stelle schließlich nicht vergessen darf, dass keiner der Fertigungs- bzw. Handhabungsschritte in einem Reinraum vorgenommen wird. Darüber hinaus werden die Messwerte aus mindestens sieben Fertigungschargen erhoben. Die vergleichsweise hohe Zahl funktionsfähiger Düsen bestätigt den vorgestellten Prozessflow. Dieser zeichnet sich durch seine intensiven Reinigungsvorgängen sowohl in Ultra- als auch in Megaschallbädern, den Spülprozessen vor dem anodischen Bonden mit Isopropanol und Aceton und auch dem Prozess der Düsenöffnung, inklusive Rückdünnung der Borosilikatglasmembran, aus.

Eine größere Biegung der Membran führt zu einem stärkeren Druckimpuls für den Tropfenausstoß. Die fluidmechanischen Untersuchungen fordern daher einen gleichbleibenden Energieeintrag während der Projektlaufzeit. Daher werden die Hübe der bimorphen piezoelektrischen Aktoren gemessen und die auftretenden Standardabweichungen betrachtet. Die Hubmessungen werden an einem Laserdopplervibrometer OFV-3001 mit angeschlossenem faseroptischem Messkopf OFV-512, beides von der Firma Polytec, auf einem schwingungs-

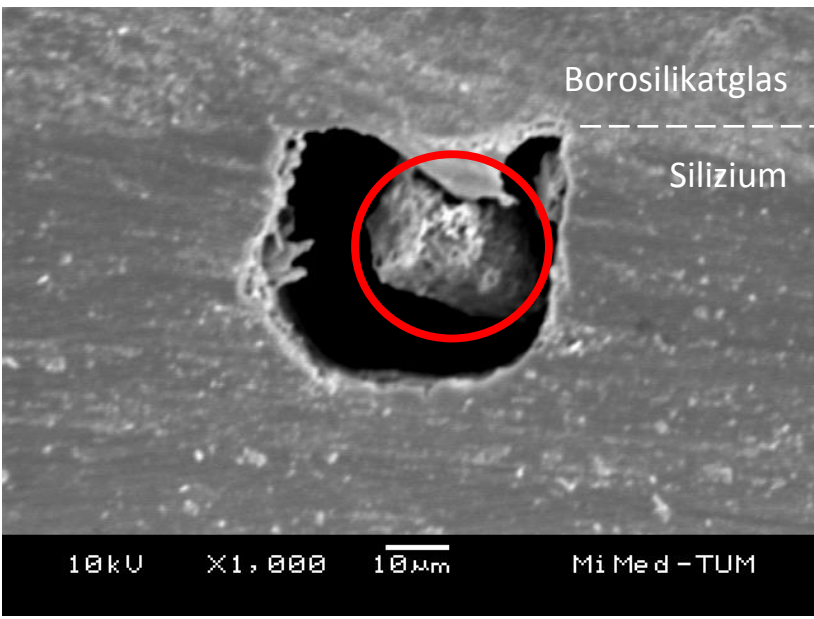

(a)

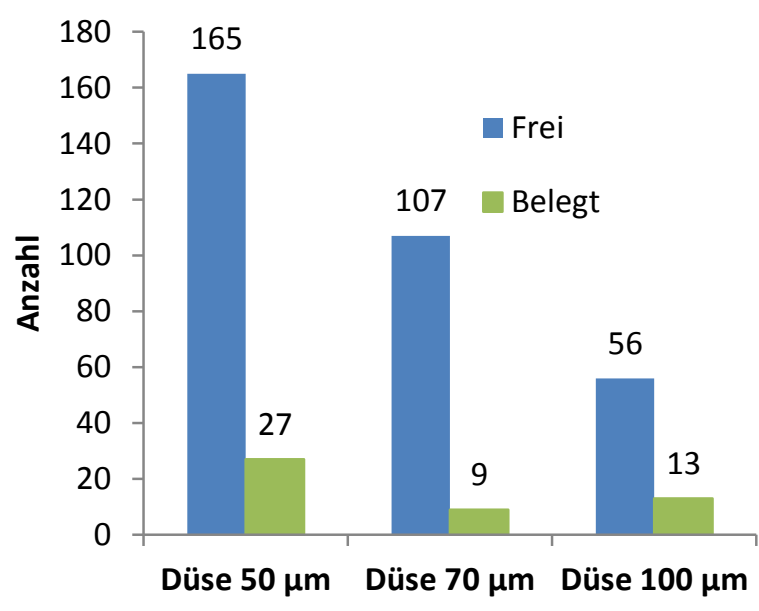

(b)

Abb. 79: (a) Eingeschlossener Partikel in einer Düse verhindert den Fluidausstoß und (b) Anzahl an funktionsfähigen, spülbaren und nicht mit Fremdkörpern besetzten Düsen. 
entkoppelten Tisch durchgeführt. Es wird ein Sinussignal mit $1 \mathrm{kHz}$ Frequenz und Amplituden zwischen 25 und $100 \mathrm{~V}$ angelegt. Es werden für drei unterschiedlich breite Elektroden zwischen 1,2 und 1,8 mm die resultierenden Hübe als Datengrundlage gemessen (siehe Abb. 80). Die Fluidkammer nimmt eine Breite von $2 \mathrm{~mm}$ ein. Die Messergebnisse werden in Abb. 81 dargestellt. Der Einsatz einer schmäleren Elektrode (Messwert: 1,2 mm) als die Standard Elektrode mit einer Breite von 1,6 mm reduziert den Hub, da die elektrisch aktive Fläche und somit die elektrische Kapazität der korrespondierenden Aktorzunge verkleinert wird. Eine breitere Elektrode (Messwert: 1,8 mm) verringert ebenfalls den Hub, obwohl die elektrisch aktive Fläche vergrößert wird. Mit zunehmender Breite der Aktorzunge wird durch die abnehmende Breite des seitlichen Festkörpergelenkes dieses steifer und seine Nachgiebigkeit nimmt ab, so dass die Membran immer mehr in ihrer Bewegung gehindert wird. Daher ergeben sich als Optimum der Breite für die Elektroden des vorliegenden Druckchipdesigns 1,6 mm. Es ist ein proportionaler Zusammenhang zwischen der angelegten elektrischen Spannung und dem resultierenden Hub aufgrund der piezoelektrischen Charakteristik ersichtlich. Die größte Standardabweichung bei den Standard Elektroden von 1,6 mm Breite tritt gleichzeitig bei der höchsten angelegten Spannungsamplitude auf. Hier werden Hübe von $176 \mathrm{~nm}$ mit einer Standardabweichung von $8 \mathrm{~nm}$ erreicht. Dabei werden die Messwerte von neun Aktorzungen aus drei Fertigungschargen gewonnen, was wiederum auf einen über den Projektzeitraum gleichbleibenden Energieeintrag des Aktors für die fluidmechanischen Untersuchungen schließen lässt.

Nachfolgend wird beispielshaft ein fluidmechanisches Dosierexperiment / -beispiel mit vorliegendem Tropfenerzeuger beschrieben. Hierbei wird ersichtlich, dass trotz ausreichender theoretischer Vorüberlegungen Funktionsmodelle in der Produktentstehung unabdingbar sind, um eine Eigenschaftsbewertung durchzuführen.

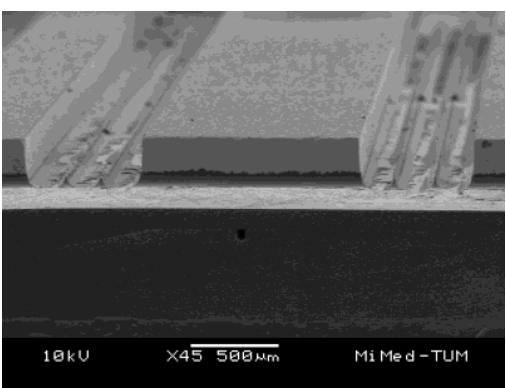

(a)

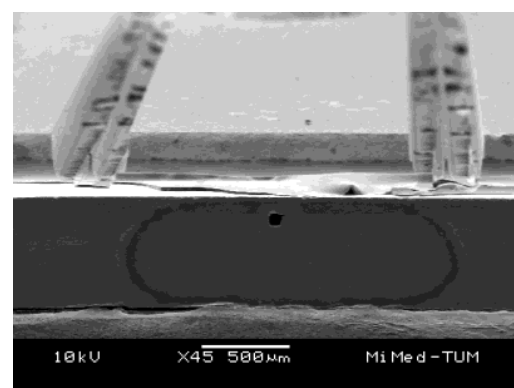

(b)

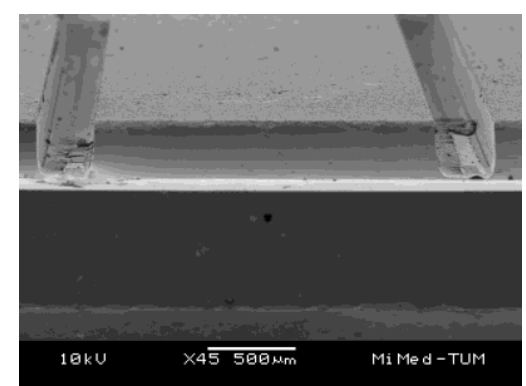

(c)

Abb. 80: Rasterelektronenmikroskopische Aufnahmen unterschiedlich breiter Elektroden des piezoelektrischen Wandlers. (a) 1,2 mm, (b) 1,6 mm und (c) 1,8 mm.

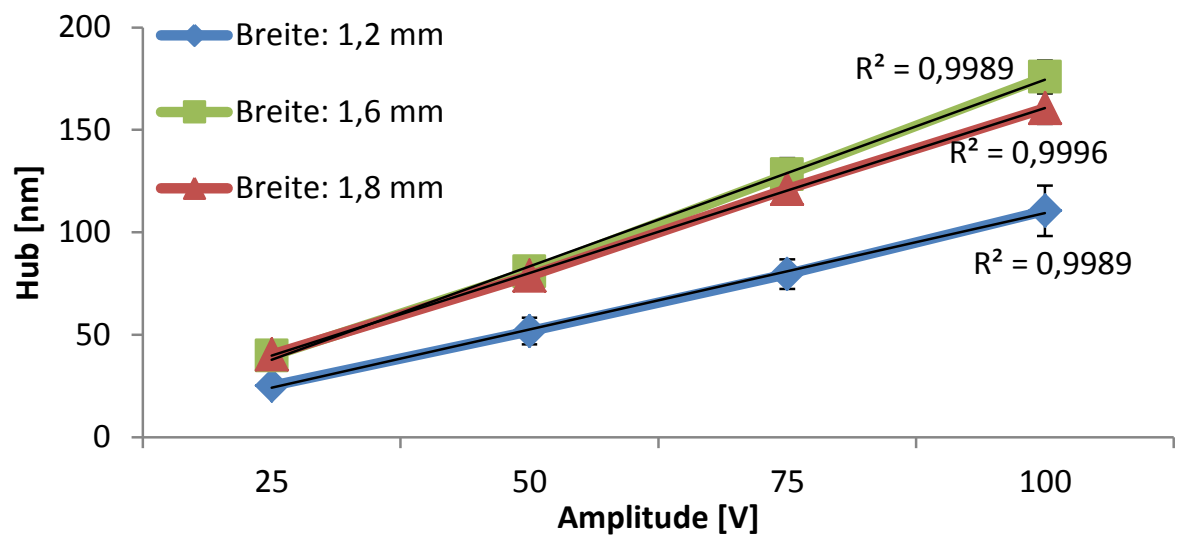

Abb. 81: Hubmessung in Abhängigkeit der elektrischen Spannung an dem Druckchip für drei unterschiedlich breite Elektroden. Messwerte entnommen aus Rumschoettel et al. (2014). 


\subsection{Fluidmechanisches Dosierexperiment / -beispiel}

\subsubsection{Beschreibung des Messverfahrens}

In diesem fluidmechanischen Dosierexperiment / -beispiel gilt es nicht nur, den Funktionsnachweis der hergestellten Mikrotropfenerzeuger zu erbringen, sondern auch aufzuzeigen, wie wichtig Funktionsmodelle in der Produktentstehung sind. Es wird vorliegend der Einfluss der Düsendimensionen auf die erzielten Parameter - Tropfenfluggeschwindigkeit, ausgestoßenes Fluidvolumen und notwendige Mindestspannung für einen Tropfenausstoß in Abhängigkeit der Pulsdauer - untersucht. Durch den umfassenden Herstellungsprozess ist es möglich, Mikrotropfenerzeuger mit unterschiedlichen Dimensionen in ausreichender Stückzahl und in kurzer Zeit zu fertigen.

Um den Einfluss der Düsendimensionen auf den Tropfenbildungsprozess aufzuzeigen, wird ein Fluid mit einer Viskosität $\eta$ von $5 \mathrm{mPa} \cdot \mathrm{s}$ und einer Oberflächenspannung $\sigma$ von $40 \mathrm{mN} / \mathrm{m}$ verwendet. Diese Eigenschaften entsprechen ansatzweise den kommerziell eingesetzten Fluiden wie bspw. Härtern für den 3D Druck. Generell werden hierbei eine hohe Tropfenfluggeschwindigkeit und ein großes ausgestoßenes Fluidvolumen verlangt. Eine hohe Tropfenfluggeschwindigkeit ist Voraussetzung für eine hohe Kantenschärfe der erstellten Bauteile in einem Sand-, Polymer- oder auch Gipsbett. Ein hohes ausgestoßenes Fluidvolumen auf dieses Pulverbett bedeutet, dass die Druckzeit pro Ebene so gering wie möglich gehalten wird.

Um ein Fluid mit definierten Eigenschaften, wie Viskosität und Oberflächenspannung, bereitzustellen, wird in Kagerer et al. (2014) ein ternäres Stoffgemisch vorgestellt, bei welchem die Oberflächenspannung und die Viskosität gezielt und unabhängig voneinander durch Wahl unterschiedlicher Stoffmengenanteile eingestellt werden. Als Grundmedium wird Glycerin verwendet. Glycerin hat eine Viskosität von ca. $1000 \mathrm{mPa} \cdot \mathrm{s}$ und eine Oberflächenspannung von ca. $64 \mathrm{mN} / \mathrm{m}$. Durch Zugabe von destilliertem Wasser $(\eta \approx 1 \mathrm{mPa} \cdot \mathrm{s} ; \sigma \approx 72 \mathrm{mN} / \mathrm{m})$ wird die Viskosität von Glycerin reduziert. Glycerin und destilliertes Wasser besitzen eine ähnlich hohe Oberflächenspannung von größer $60 \mathrm{mN} / \mathrm{m}$. Die Beimischung von Isopropanol $(\eta \approx 2 \mathrm{mPa} \cdot \mathrm{s} ; \sigma \approx 21 \mathrm{mN} / \mathrm{m})$ ermöglicht eine Herabsetzung der Oberflächenspannung des Glycerin / Wasser Gemisches. Um ein Fluid mit einer Viskosität von $5 \mathrm{mPa} \cdot \mathrm{s}$ und einer Oberflächenspannung von $40 \mathrm{mN} / \mathrm{m}$ zu mischen, werden folgende Stoffmengen bezogen auf $10 \mathrm{~g}$ Fluidgemisch benötigt: Wasser 5,05 g, Glycerin 4,4 g und Isopropanol 0,55 g.

Um den Einfluss der Düsenbreite auf die Tropfenparameter erkennbar zu machen, werden Düsen mit einer Breite von 50, 70 und $100 \mu \mathrm{m}$ bei einer konstanten Düsenlänge von $100 \mu \mathrm{m}$ eingesetzt. Um den Einfluss der Düsenlänge auf die Tropfenparameter aufzuzeigen, werden Düsen mit einer Länge von 50, 100 und $200 \mu \mathrm{m}$ bei einer konstanten Düsenbreite von $70 \mu \mathrm{m}$ eingesetzt. Alle weiteren Dimensionen der Mikrotropfenerzeuger entsprechen den bislang beschriebenen Größen. Dies bedeutet, dass alle weiteren fluidmechanischen Strukturen $100 \mu \mathrm{m}$ tief sind, die Drossel eine Breite von $200 \mu \mathrm{m}$ bei einer Länge von $1 \mathrm{~mm}$ einnimmt, die Fluidkammer $2 \mathrm{~mm}$ breit und $10 \mathrm{~mm}$ lang ist und die Elektrodenzungen auf dem piezoelektrischem Wandler 1,6 mm breit sind. Es werden jeweils die notwendige Mindestspannung in Abhängigkeit der Pulsdauer für einen Tropfenausstoß und sowohl das ausgestoßene Fluidvolumen als auch die Tropfenfluggeschwindigkeit bei fester elektrischer Ansteuerung bestimmt. Die Ansteuerfrequenz ist während den Untersuchungen mit $200 \mathrm{~Hz}$ konstant.

Die Versuche werden an einem stroboskopischen Tropfenbeobachtungsstand durchgeführt, welcher neben der Beobachtung einzelner ausgestoßener Tropfen auch die Messung von 
Tropfenfluggeschwindigkeit und -durchmesser automatisiert durchführt. Aufgrund von Oberflächenkräften kann es zu einer Teilung des Tropfens in einen Haupt- und in einen Satellitentropfen kommen. Daher wird an dieser Stelle nicht der Tropfendurchmesser, sondern das gesamte ausgestoßene Fluidvolumen zur Bewertung herangezogen. (11.1) gibt die Berechnung des ausgestoßenen Fluidvolumens $\mathrm{V}_{\text {Fluid }}$ mit dem Durchmesser des Haupt- $\mathrm{d}_{\text {Haupt }}$ und des Satellitentropfens $d_{\text {Satellit }}$ wieder. Die Messung der Tropfen wird ca. $400 \mu$ s nach elektrischer Ansteuerung durchgeführt und es wird der Mittelwert aus mindestens drei Düsen gebildet.

$$
V_{\text {Fluid }}=\frac{4}{3} \cdot \pi \cdot\left(\left(\frac{d_{\text {Haupt }}}{2}\right)^{3}+\left(\frac{d_{\text {Satellit }}}{2}\right)^{3}\right)
$$

\subsubsection{Experiment zur Messung der Vorteile}

Die Untersuchungen werden an einem stroboskopischen Beobachtungsstand mit integrierter Messmöglichkeit vorgenommen (siehe Abb. 82). Der Tropfenausstoß stellt dabei einen periodischen Vorgang dar. Hierdurch ist es möglich, durch das periodensynchrone stroboskopische Blitzen einzelne Tropfen in Zeitlupe bzw. in verschiedenen Tropfenentstehungsphasen zu beobachten. Hierzu werden mehrere aufeinander ausgegebene Tropfen zu einer fest definierten Zeit nach ihrem Ausstoß aus der Düse von einem Lichtblitz beleuchtet, so dass sich ein scheinbar stehendes Tropfenbild ergibt. Über ein Programm am Steuerrechner des Tropfenbeobachtungsstandes können im Anschluss sowohl Tropfendurchmesser als auch -fluggeschwindigkeit bestimmt werden. Vor den fluidmechanischen Experimenten wird das Arbeitsfluid in einem Ultraschallbad entgast, um die Anzahl an störenden Luftblasen zu minimieren. Im Anschluss erfolgt ein Spülvorgang des Mikrotropfenerzeugers, welcher die fluidmechanischen Komponenten blasenfrei mit dem Arbeitsfluid füllt. Da vorliegend mit Flüssigkeiten gearbeitet wird, kommt als Fluidreservoir eine Einwegspritze zur Verwendung. Nach dem Spülvorgang wird das Fluidreservoir unterhalb der Düse positioniert, um einen Unterdruck im Dosiersystem zu erhalten. Hierzu wird die Flüssigkeitssäule des Fluidreservoirs mit einem Höhenunterschied von 3 bis $4 \mathrm{~cm}$ unterhalb der Düsenöffnung positioniert. Die Mikrotropfenerzeuger werden mit einem positiven Rechteckimpuls angesteuert. Es werden ein Funktionsgenerator der Firma Agilent (Modell 33220A) und ein Spannungsverstärker der Firma Trek (Modell PZD 350A) eingesetzt.

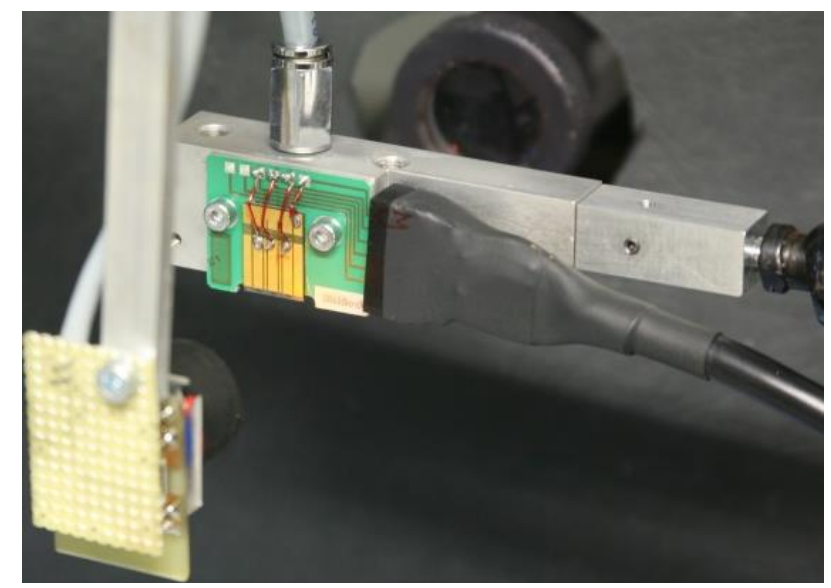

(a)

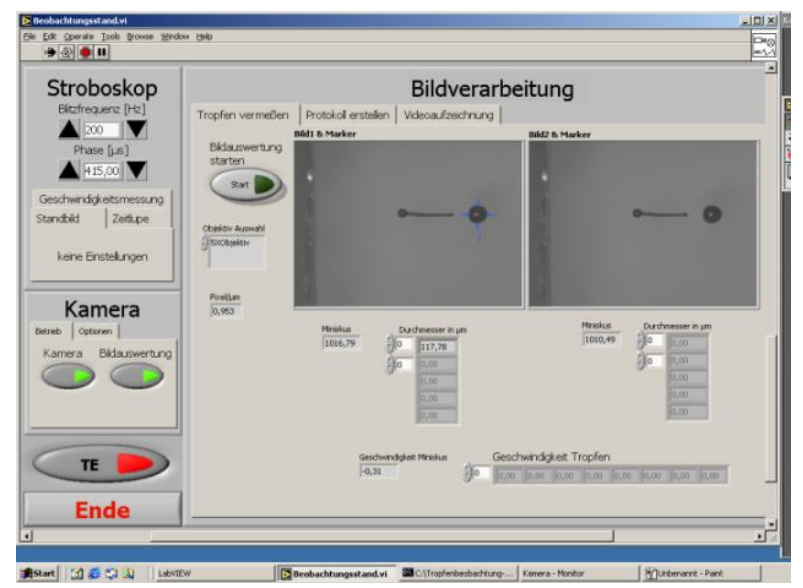

(b)

Abb. 82: (a) Aufnahmevorrichtung mit Mikrotropfenerzeuger in dem stroboskopischen Tropfenbeobachtungsstand und (b) Auswertesoftware des stroboskopischen Tropfenbeobachtungsstandes mit Satellitentropfenaufnahme. 
Abb. 83 gibt die Ergebnisse unter Variation der Düsenbreite wieder. Abb. 83 (a) zeigt dabei die benötigte Mindestspannung in Abhängigkeit der Pulsdauer für einen Tropfenausstoß. Das Ergebnis ist, dass ab einer Pulsdauer von $50 \mu$ s ein gesättigter Bereich auftritt, bei dem zwischen den einzelnen Düsenbreiten keine signifikanten Spannungsunterschiede auftreten. Es ist mit allen drei Düsenbreiten möglich, Tropfen ab $30 \mathrm{~V}$ Ansteueramplitude aus der Düse zu stoßen. Im Ergebnis bedeutet dies, dass der Einfluss der Düsenbreite auf die benötigte Mindestspannung in dem gesättigten Bereich als gering einzuschätzen ist. Es ist zu erkennen, dass bei kurzen Pulsen die benötigte Mindestspannung zum Ausstoß eines Tropfens wächst, und dass die breiteste Düse mit einer Breite von $100 \mu \mathrm{m}$ die höchste Ansteueramplitude, vor allem bei kurzen Pulsdauern, benötigt. In Abb. 83 (b) ist der Verlauf der Tropfenfluggeschwindigkeit über die drei Düsenbreiten bei zwei Ansteueramplituden aufgetragen. Es ist den Messwerten zu entnehmen, dass sowohl die breiteste als auch die schmälste Düse zu niedrigeren Tropfenfluggeschwindigkeiten führen. Die maximalen Tropfenfluggeschwindigkeiten werden bei einer Düsenbreite von $70 \mu \mathrm{m}$ erzielt. Aufgrund der piezoelektrischen Eigenschaft gilt, je höher die Ansteueramplitude ist, desto schneller ist auch die Tropfenfluggeschwindigkeit, da die Biegung des piezoelektrischen Aktors ebenfalls steigt. Erwartungsgemäß steigt das ausgestoßene Fluidvolumen mit breiter werdender Düse aufgrund der größeren Durchgangslücke (siehe Abb. 83 (c)).

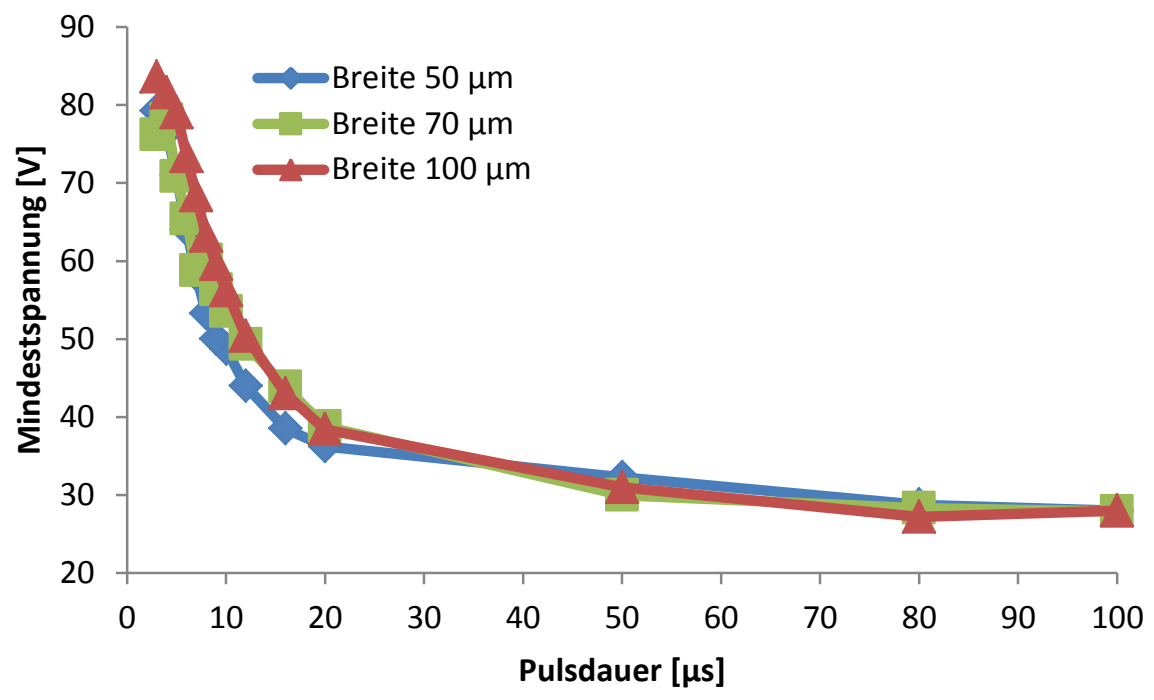

(a)

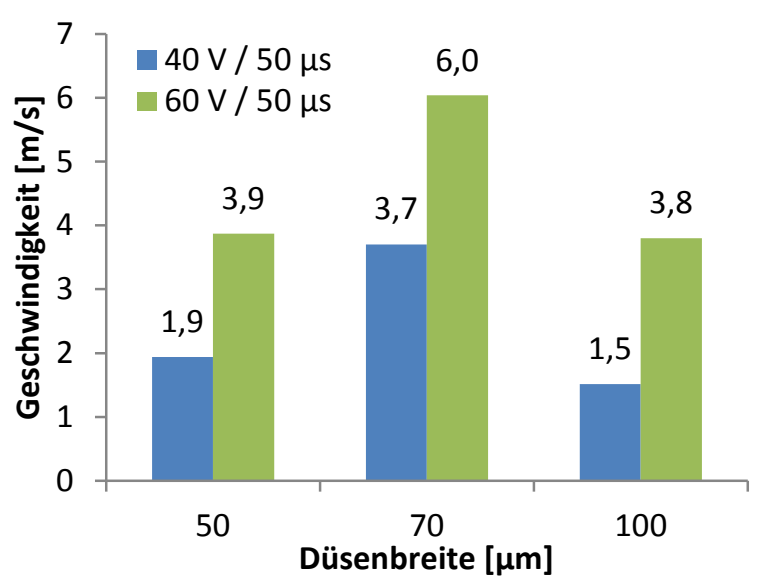

(b)

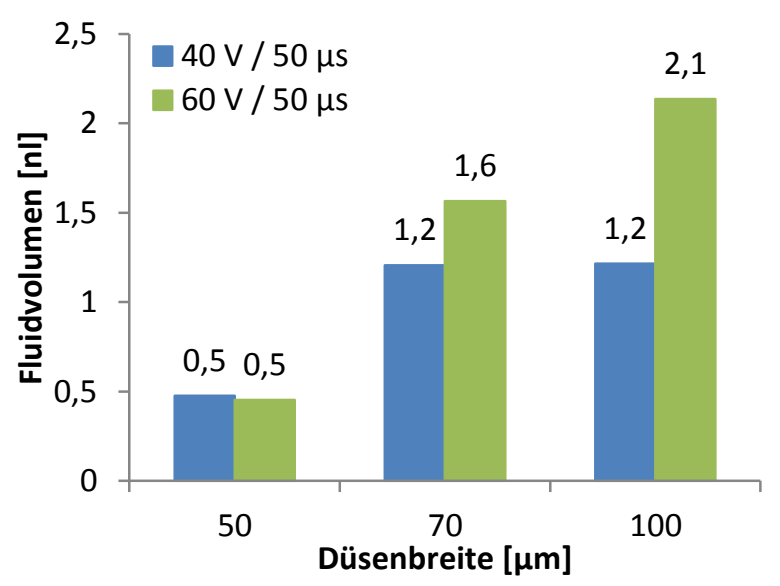

(c)

Abb. 83: Einfluss der Düsenbreite bei konstanter Düsenlänge von $100 \mu \mathrm{m}$ auf (a) notwendige Mindestspannung für Tropfenflug, (b) auftretende Tropfenfluggeschwindigkeit und (c) ausgestoBenes Fluidvolumen. Messwerte entnommen aus Kagerer et al. (2014). 
Abb. 84 gibt die Ergebnisse bei Variation der Düsenlänge wieder. Die Ergebnisse der Untersuchungen zeigen, bei längeren Düsen wird mehr Energie für einen Tropfenausstoß benötigt (siehe Abb. 84 (a)). Grund hierfür stellt der damit verbundene größere fluidmechanische Widerstand dar. Die längste Düse erzielt aufgrund der höchsten Reibung die geringsten Tropfenfluggeschwindigkeiten. Die schnellsten Tropfen werden aus der Düse mit einer Länge von $100 \mu \mathrm{m}$ ausgestoßen (siehe Abb. 84 (b)). Dies lässt darauf schließen, dass eine gewisse Mindestlänge der Düse notwendig ist, um das Fluid im Düsenkanal ausreichend zu beschleunigen. Der zweite Tropfenparameter ist das ausgestoßene Fluidvolumen. Abb. 84 (c) stellt dar, wie sich das Fluidvolumen über der Länge der Düse verhält. So bietet die Düse mit einer Länge von $100 \mu \mathrm{m}$ das höchste ausgestoßene Fluidvolumen. Der sehr geringe Volumenstrom der längsten Düse ist erklärbar durch den erhöhten Strömungswiderstand.

An dieser Stelle wird vor allem deutlich, wie wichtig kostengünstige, individuelle und rasch herstellbare Funktionsmodelle sind. (11.2) zeigt die Gesamtenergie $\mathrm{E}_{\mathrm{Ges}}$, welche für einen Tropfenflug notwendig ist. Diese setzt sich dabei aus einem Anteil $\mathrm{E}_{\text {Visk }}$ zur Überwindung der Reibung im Düsenkanal, einem Anteil $E_{\text {Oberf }}$ zur Formung des Tropfens und einem Anteil $E_{\text {Kin }}$ für den Tropfenflug zusammen. Hierbei sind $v_{\text {Düse }}$ die Geschwindigkeit in der Düse, $1_{\text {Düse }}$ die Düsenlänge, $r_{\text {Tropf }}$ der Tropfenradius, $v_{\text {Tropf }}$ die Tropfenfluggeschwindigkeit, $\eta$ die Viskosität,

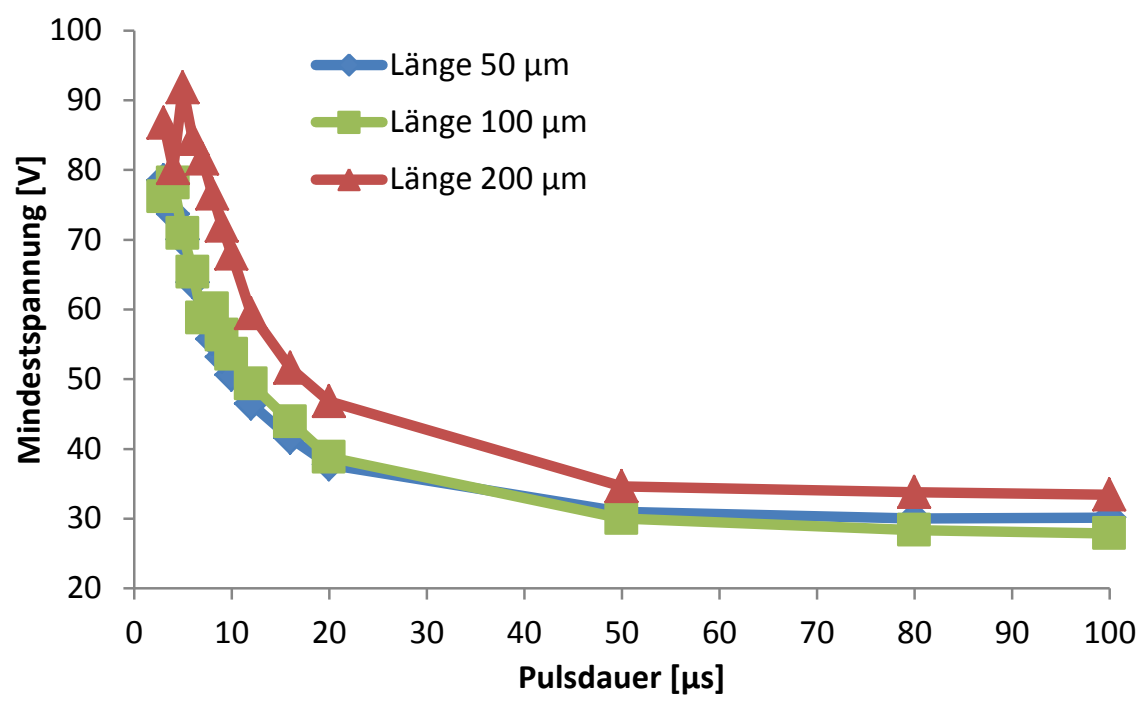

(a)

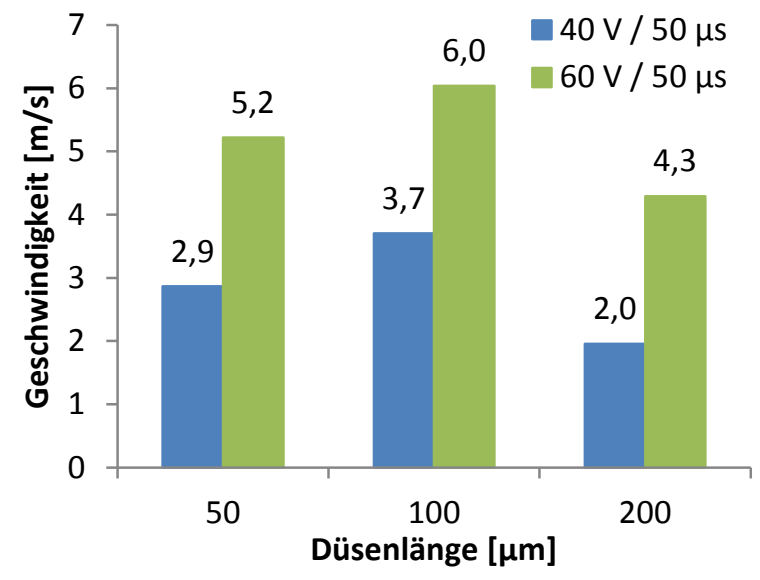

(b)

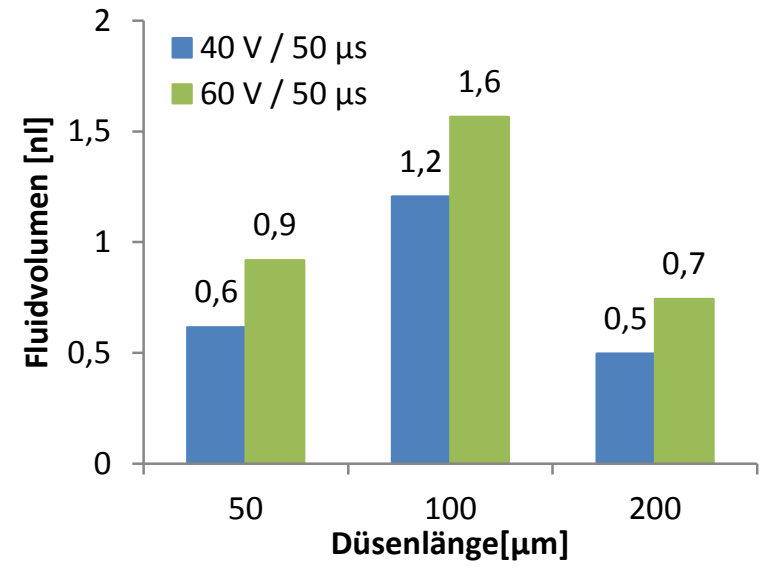

(c)

Abb. 84: Einfluss der Düsenlänge bei konstanter Düsenbreite von $70 \mu \mathrm{m}$ auf (a) notwendige Mindestspannung für Tropfenflug, (b) auftretende Tropfenfluggeschwindigkeit und (c) ausgestoßenes Fluidvolumen. Messwerte entnommen aus Kagerer et al. (2014). 
$\sigma$ die Oberflächenspannung und $\rho$ die Dichte des Arbeitsfluides. Entgegen den theoretischen Erwartungen werden bei identischem Energieeintrag die höchsten Tropfenfluggeschwindigkeiten nicht bei den schmälsten und kürzesten Düsen erzielt, obwohl hier geringe Fluidvolumina und somit auch geringe Tropfendimensionen erreicht werden. Dies bedeutet, dass eine gewisse Mindestbreite bzw. -länge einer Düse notwendig ist, um das Fluid im Kanal ausreichend zu beschleunigen. Dieses Ergebnis konnte nur durch den Einsatz vorliegender Funktionsmodelle aufgezeigt werden.

$$
\begin{aligned}
& E_{\text {Ges }}=E_{\text {Visk }}+E_{\text {Oberf }}+E_{\text {Kin }}= \\
& =8 \cdot v_{\text {Dise }} \cdot \pi \cdot \eta \cdot l_{\text {Dise }}^{2}+4 \cdot \pi \cdot r_{\text {Tropf }}^{2} \cdot \sigma+\frac{1}{2} \cdot \rho \cdot \frac{4}{3} \cdot \pi \cdot r_{\text {Tropf }}^{3} \cdot v_{\text {Tropf }}^{2}
\end{aligned}
$$

\subsubsection{Zusammenfassung der Ergebnisse}

Durch vorliegendes Dosierbeispiel wird gezeigt, wie sich die Tropfenparameter - die notwendige Mindestspannung für den Tropfenausstoß in Abhängigkeit der Pulsdauer und sowohl das ausgestoßene Fluidvolumen als auch die Tropfenfluggeschwindigkeit - bei Variation der Düsenbreite und -länge ändern. Es ergibt sich, dass bereits ab $30 \mathrm{~V}$ Ansteueramplitude unabhängig von der Düsendimension Tropfen ausgestoßen werden. Bei kleinen Pulsdauern ergibt sich ein stark ansteigender Zusammenhang zwischen Mindestspannung und Pulsdauer. Entgegen den Erwartungen werden die höchsten Tropfenfluggeschwindigkeiten weder bei der kürzesten noch bei der schmälsten Düse erzielt. Es wird deutlich, dass eine gewisse Mindestbreite wie auch -länge der Düse notwendig ist, um das Fluid im Düsenkanal ausreichend zu beschleunigen. Dieses Resultat ist nur durch den Einsatz von Funktionsmodellen belegt worden und kann nicht durch die Gesamtenergiebilanz aus (11.2) abgeleitet werden. Es wird an dieser Stelle ersichtlich, wie wichtig Funktionsmodelle in der Produktentstehung sind. Es können schnelle Tropfen mit einer Fluggeschwindigkeit von mindestens $5 \mathrm{~m} / \mathrm{s}$ mit einem großen ausgestoßenem Fluidvolumen von mindestens 1,5 nl erzeugt werden. Abb. 85 zeigt stroboskopische Aufnahmen des Tropfenbildungsprozesses. Dieser zeigt auch das Phänomen der Satellitentropfenbildung aufgrund von Oberflächenkräften auf.

Des Weiteren sei an dieser Stelle auch erwähnt, dass mit vorliegendem Mikrotropfenerzeuger bereits erfolgreich Kerzenwachs dosiert worden ist. Dieses ist in dem Fluidreservoir aufgeschmolzen (ca. $80^{\circ} \mathrm{C}$ ) und im flüssigen Zustand dosiert worden (Kagerer et al., 2012b). Es ist damit sowohl der Funktionsnachweis der hergestellten Mikrotropfenerzeuger erbracht als auch aufgezeigt, wie wichtig Funktionsmodelle in der Produktentstehung sind.

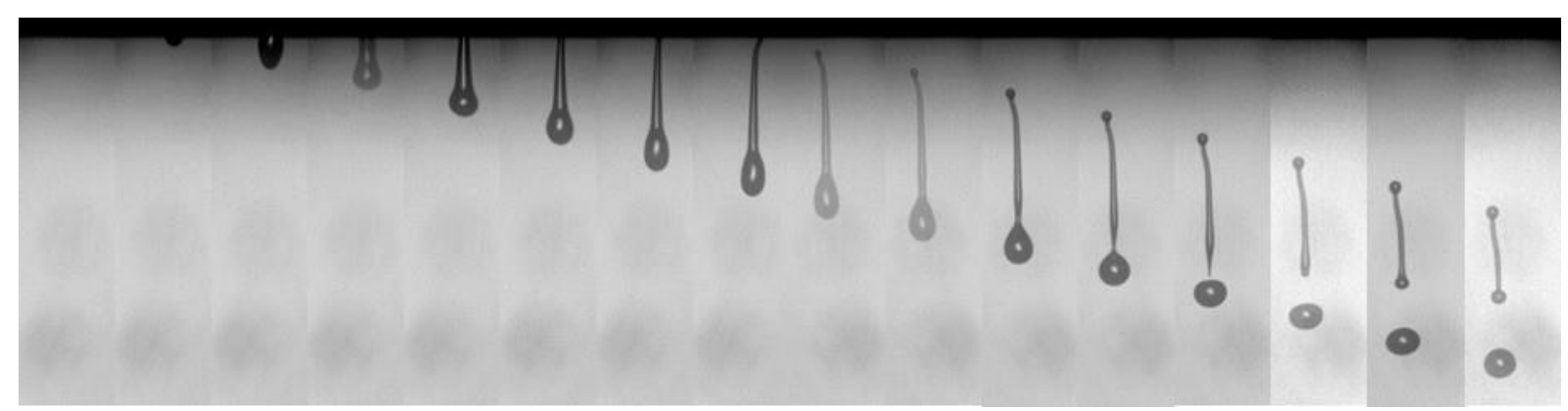

Abb. 85: Exemplarische stroboskopische Aufnahme des Tropfenbildungsprozesses mit Satellitentropfenbildung. Die Düse hat hierbei einen Querschnitt von $70 \times 70 \mu \mathrm{m}^{2}$ mit einer Länge von $50 \mu \mathrm{m}$. Ansteuerung mit $40 \mathrm{~V}$ und einer Pulsdauer von $50 \mu \mathrm{s}$. Abstand der Bilder je $20 \mu \mathrm{s}$. 


\section{Zusammenfassung und Ausblick}

Die vorliegende Arbeit löst die Aufgabe, individuelle Funktionsmodelle / Redesigns für die Mikrosystemtechnik in geringen Stückzahlen kostengünstig und auch in kurzer Zeit zu fertigen. Hiermit ist es bereits in frühen Phasen der Entstehung eines Mikrosystems möglich, die geforderten Produkteigenschaften abzusichern. Die meisten Mikrosysteme besitzen eine hybride Architektur. Dies bedeutet, dass diese aus einem strukturierten Substrat und mindestens aus einem hierauf montierten Funktionswerkstoff bestehen. Bei dem vorliegenden Anwendungsbeispiel eines piezoelektrisch betriebenen Mikrotropfenerzeugers sind die fluidmechanischen Strukturen in ein Siliziumsubstrat eingebracht und hierauf ist ein piezoelektrischer Aktor montiert. Somit ergeben sich für die Fertigung von Funktionsmodellen für die Mikrosystemtechnik immer zwei Aufgaben. Die Mikrostrukturierung von Substraten und die Mikromontage für die Fügung von Bauteilen. Im Rahmen dieser Arbeit ist der Fokus auf die Mikrostrukturierung von Silizium und auf das Kleben als eingesetztes Fügeverfahren gelegt.

Vorliegend werden für die Mikrostrukturierung von Siliziumsubstraten sowohl die Laserstrahlmaterialbearbeitung als auch das Trennschleifen eingesetzt. Das Trennschleifen mit einer Wafersäge wird in dieser Arbeit für die Erstellung senkrechter Kanäle mit geringster Rauheit eingesetzt. Dies senkt vor allem bei fluidmechanischen Anwendungen die auftretende Reibung des Fluides im Kanal. Die Laserstrahlmaterialbearbeitung zeichnet sich dadurch aus, dass es nahezu keine Einschränkung in der Formenvielfalt gibt, unterschiedliche Designvariationen in CAD Programmen erstellt und direkt in die Lasersystemsteuerung importiert werden können, und dass vor allem keine Photolithographie erforderlich ist. Neben Parametern, welche direkt von der Laserstrahlquelle anhängig sind, beeinflussen auch weitere grundlegende Bearbeitungsparameter das Abtragsergebnis, bewertet nach Abtragsrate, Formtreue und auch auftretender Rauheit. Im Rahmen dieser Arbeit ist hierzu das Zusammenspiel der Parameter Fokusposition, Vorschubgeschwindigkeit und auch Linienabstand sowohl auf das Laserstrahlschneiden als auch auf den ebenen Laserstrahlmaterialabtrag aufgezeigt. Diese Parameter sind nicht von der Laserstrahlquelle abhängig, so dass die aufgezeigten Zusammenhänge auf jedes Lasersystem übertragen werden können.

Im Gegensatz zu dem standardisierten Heraustrennen von Chips aus Substraten mit dem Trennschleifprozess mit einer Wafersäge werden im Rahmen der vorliegenden Arbeit Kanäle mit definierter Tiefe und Breite in Siliziumsubstraten erstellt. Hierfür ist ein zweistufiger Prozess entwickelt, welcher zum einen auf der Bestimmung der Lage der Substratoberfläche beruht und zum anderen überlappende Sägeschnitte verwendet, damit Kanäle unabhängig von der Sägeblattbreite erstellt werden.

Für die notwendige Fügung von zwei Bauteilen ist ein Mikromontageplatz erstellt, welcher für die Hauptanwendung der Fügung von planaren Substraten über eine dünne, homogene und ganzflächige Klebstoffschicht entwickelt worden ist. Der Mikromontageplatz zeichnet sich durch einen kompakten und vor allem modularen Aufbau aus. Das abzusetzende Bauteil wird über einen Vakuumsauggreifer, welcher an einem Hubkolben angebracht ist, aufgenommen. Die zugrundeliegende Kinematik des Hubkolbens entspricht dabei einem Schubkurbelgetriebe. Das Zielbauteil / Substrat befindet sich auf einer Montageplattform, welche sowohl drei translatorische als auch einen rotatorischen Freiheitsgrad für die Ausrichtung beider Bauteile zueinander besitzt. Zusätzlich ist eine Heizwicklung integriert, so dass Aushärtevorgänge von 
Klebschichten beschleunigt werden. Um homogene und ganzflächige Klebstoffschichten auf Substraten zu erzielen, ist ein Klebstoffauftragswerkzeug an dem Mikromontageplatz integriert. Das Arbeitsprinzip entspricht dabei dem Abziehen von Klebstoff auf dem Zielbauteil mit einer Rakelklinge. Über Haltearme können weitere Werkzeuge, wie bspw. Videomikroskope oder Messtaster, an dem Mikromontageplatz angebracht werden.

Die aufgezeigten Fertigungsverfahren werden erfolgreich bei Herstellung von Mikrotropfenerzeugern eingesetzt. Es werden insgesamt fünf identische Mikrotropfenerzeuger gleichzeitig gefertigt, so dass eine ausreichend hohe Anzahl an Funktionsmodellen in der Produktentstehung für die Eigenschaftsbewertung vorliegt. Diese werden in weniger als 10 Stunden gefertigt. Die kurze Zeit ist nur möglich, da auf die langwierige mikroelektronische Fertigungstechnik mit alternierender Prozessfolge verzichtet wird, die einzelnen Bearbeitungsparameter bei der Mikrostrukturierung gezielt aufeinander abgestimmt worden sind und auch die intuitive Bedienung des Mikromontageplatzes mit geringen Rüstzeiten gewährleistet ist. Zusätzlich ist diese Art der Fertigung sehr kostensparend, da im Vergleich zu der mikroelektronischen Fertigungstechnik weder eine Vielzahl an Gerätschaften noch unterschiedliche Chemikalien, Maskensätze und auch kein Reinraum benötigt werden. Neben der erfolgreichen zeitlichen Evaluation konnte auch die Prozessgenauigkeit bewertet werden. Alle gestellten Anforderungen an die Fertigungsgenauigkeit aus Kapitel 4 konnten im Rahmen dieser Arbeit erfolgreich erfüllt werden.

In dem beschriebenen fluidmechanischen Dosierbeispiel unter der Variation der Düsendimension zeigte sich, wie essentiell Funktionsmodelle in der Eigenschaftsbewertung von Mikrosystemen sind. Entgegen den theoretischen Betrachtungen werden weder bei der kürzesten noch bei der schmalsten Düse die höchsten Tropfenfluggeschwindigkeiten erzielt. Es zeigt sich, dass sowohl eine gewisse Mindestbreite als auch -länge der Düse notwendig ist, um das Fluid im Düsenkanal ausreichend zu beschleunigen. Dieses Resultat konnte nur durch den Einsatz von Funktionsmodellen erzielt werden.

Es ergeben sich durch die vorliegende Arbeit folgende Vorteile für die Mikrosystemtechnik:

- Bereitstellung von Fertigungsverfahren, mit denen eine angepasste und kostengünstige Fertigung von Mikrosystemen in geringen Stückzahlen möglich ist

- Integration von Funktionsmodellen in den Produktentstehungsprozess von Mikrosystemen ist in effizienter Weise möglich. Hierdurch werden Fehler im Entwurf zu einem frühen Zeitpunkt erkannt und ein mögliches Redesign rasch abgeleitet

- Durchführung von Kalibrierprozessen an Mikrosystemen oder auch an Kompakt- / Minimalmodellen ist möglich

- Erschließung neuer Märkte für die Mikrosystemtechnik ist möglich, da die großen Hindernisse, wie bspw. Investitionskosten und auch die komplexe Prozessführung zur Fertigung neuer Mikrosysteme, nicht im Vordergrund stehen

- Verringerung des Schulungsaufwandes von Operatoren, da im Vergleich zu der mikroelektronischen Herangehensweise nur in geringem Maße das komplexe mikrotechnologische Fachwissen, um bspw. einzelne Prozessschritte für die Mikrostrukturierung aufeinander abzustimmen, benötigt wird 
Ausgehend von vorliegender Arbeit ergeben sich Möglichkeiten für weitere Arbeiten. Bislang verfügt der entwickelte Mikromontageplatz über manuell verfahrbare Achsen mit Mikrometerstellschrauben an der Montageplattform. Der maximale Verfahrweg beträgt $50 \mathrm{~mm}$. Dies bedeutet, dass sowohl die maximale Auftragsfläche für den Klebstoff als auch die maximale Distanz bei Relativpositionierungen hiervon begrenzt werden. Um beide Werte zukünftig zu vergrößern und gleichzeitig die Positioniergenauigkeit zu erhöhen, können motorbetriebene Achsen zum Einsatz kommen. Die getätigten Mikrostrukturierungsuntersuchungen sind an Silizium durchgeführt. Auch wenn Silizium der meist eingesetzte Werkstoff in der Mikrosystemtechnik ist, so gibt es dennoch weitere Substratmaterialien, wie bspw. Gläser, Quarze, Keramiken, Metalle und Kunststoffe. Auch hier besteht die Möglichkeit, die aufgezeigten Zusammenhänge anzuwenden. Das Ergebnis würde eine umfassende Datenbasis in Analogie zu standardisierten Ätzdreiecken aus der mikroelektronischen Fertigungstechnik für unterschiedliche Materialien bzgl. Abtragsraten, Bearbeitungsdurchmesser oder auch auftretender Rauheit sein. Bislang sind die vorgestellten Verfahren an einem piezoelektrisch betriebenen Mikrotropfenerzeuger angewendet worden. Es wäre an dieser Stelle interessant, diese Fertigungsverfahren auch bei anderen Mikrosystemen in deren Fertigungskette zu integrieren. Vor allem die Laserstrahlmaterialbearbeitung bietet hierzu einen großen Vorteil. Es ist ein direkt schreibendes Verfahren, so dass auf photolithographische Prozesse verzichtet wird und bei Substraten mit bereits aufgebrachten Funktionskomponenten ohne weiteren Chemikalieneinsatz die nachträgliche Erstellung einzelner Formen bzw. Strukturen an definierten Positionen ermöglicht wird.

Zusammenfassend gilt, dass mit vorliegender Arbeit individuelle Funktionsmodelle eines Mikrosystems in geringen Stückzahlen und gleichzeitig in kurzer Zeit fertigbar sind, so dass bereits in frühen Phasen der Entstehung die geforderten Produkteigenschaften abgesichert werden können. Es steht ein kostengünstiger Fertigungsprozess zur Verfügung, welcher weder die Umgebung noch die maskenbasierte Prozesskette der mikroelektronischen Fertigungstechnik benötigt. 


\section{Literatur}

Abgrall, P. und Gué, A. M. 2007, "Lab-on-chip technologies: making a microfluidic network and coupling it into a complete microsystem - a review", Journal of Micromechanics and Microengineering, vol. 17, no. 5, S. 15-49.

Agnus, J., Nectoux, P. und Chaillet, N. 2005, "Overview of Microgrippers and Design of a Micromanipulation Station Based on a MMOC Microgripper", Proceedings IEEE International Symposium on Computational Intelligence in Robotics and Automation (CIRA), Espoo, Finland, S. 117-123.

Albrecht, J. 2012, Inbetriebnahme einer Positioniereinheit mit Erstellung eines bedienerfreundlichen Interfaces für ein Lasersystem, Semesterarbeit, Technische Universität München.

Amer, M. S., El-Ashry, M. A., Dosser, L. R., Hix, K. E., Maguire, J. F. und Irwin, B. 2005, "Femtosecond versus nanosecond laser machining: comparison of induced stresses and structural changes in silicon wafers", Applied Surface Science, vol. 242, no. 1-2, S. 162-167.

Ancona, A., Döring, S., Jauregui, C., Röser, F., Limpert, J., Nolte, S. und Tünnermann, A. 2009, "Femtosecond and picosecond laser drilling of metals at high repetition rates and average powers", Optics Letters, vol. 34, no. 21, S. 3304-3306.

arteos 2009, TOMM 1: Mikromontage von kleinen Stückzahlen, arteos, S. 1-2. Verfügbar unter: <http://www.arteos.com/app/download/5785371620/TOMM+1+Flyer.pdf>. [24.01.2015].

Ashida, K., Nakano, S., Park, J. und Akedo, J. 2010, "On-Demand MEMS Device Production System by Module-Based Microfactory", International Journal of Automation Technology, vol. 4, no. 2, S. 110-116.

Bardeen, J. und Brattain, W. H. 1948, "The Transistor, A Semi-Conductor Triode", Physical Review, vol. 74, no. 2, S. 230-231.

Bäuerle, D. 2009, Laser: Grundlagen und Anwendungen in Photonik, Technik, Medizin und Kunst, WILEY-VCH Verlag, Weinheim, ISBN 9783527408037.

Benilov, A., Skryshevsky, V., Robach, Y. und Cabrera, M. 2008, "Micro and nano electrical discharge machining in microfluidics and micro nanotechnology", International Journal of Material Forming, vol. 1, no. 1, S. 1315-1318.

Bobzin, K., Bührig-Polazcek, A., Haberstroh, E., Michaeli, W., Poprawe, R., Reisgen, U., Flock, D., Grönlund, O., Jakob, M., Kutschmann, P., Neuß, A., Rösner, A., Scheik, S., Schleser, M. und Wunderle, J. 2011, "Verkürzung von Prozessketten bei der Herstellung von Kunststoff / Metall-Hybriden durch neuartige Urform- und Fügeprozesse", in Integrative Produktionstechnik für Hochlohnländer, Hrsg. Brecher, C., Springer, Heidelberg, Dordrecht, London, New York, Kapitel 5.3, S. 514-590, ISBN 9783642206924. 
Bogue, R. 2011, "Assembly of 3D micro-components: a review of recent research", Assembly Automation, vol. 31, no. 4, S. 309-314.

Brousseau, E. B., Dimov, S. S. und Pham, D. T. 2010, "Some recent advances in multimaterial micro- and nano-manufacturing", International Journal of Advanced Manufacturing Technology, vol. 47, no. 1-4, S. 161-180.

Brück, R., Rizvi, N. und Schmidt, A. 2001, Angewandte Mikrotechnik: LIGA-LaserFeinwerktechnik, Carl Hanser Verlag, München, ISBN 9783446214712.

Bundesministerium für Bildung und Forschung, 28.01.2014, Mikrosystemtechnik. Verfügbar unter: <http://www.bmbf.de/archiv/newsletter/de/5701.php?hilite=mikrosystemtechnik>. [24.06.2014].

Cecil, J., Powell, D. und Vasquez, D. 2007, "Assembly and manipulation of micro devices - A state of the art survey", Robotics and Computer-Integrated Manufacturing, vol. 23, no. 5 , S. 580-588.

Chien, C. Y. und Gupta, M. C. 2005, "Pulse width effect in ultrafast laser processing of materials", Applied Physics A, vol. 81, no. 6, S. 1257-1263.

Clariant 1997, AZ ${ }^{\circledR} 9200$ Photoresist: High-Resolution Thick Resist, Clariant, S. 1-8. Verfügbar unter: <http://www.microchemicals.com/micro/az_9200.pdf>. [24.04.2015].

Dauer, S., Ehlert, A. und Büttgenbach, S. 1999, "Rapid prototyping of micromechanical devices using a Q-switched Nd:YAG laser with optional frequency doubling", Sensors and Actuators A: Physical, vol. 76, no. 1-3, S. 381-385.

Dilthey, U. und Brandenburg, A. 2005, "Einleitung", in Montage hybrider Mikrosysteme: Handhabungs- und Fügetechniken für die Klein- und Mittelserienfertigung, Hrsg. Dilthey, U. \& Brandenburg, A., Springer, Berlin, Heidelberg, New York, Kapitel 1, S. 1-4, ISBN 3540237062.

Dilthey, U., Brandenburg, A. und Schleser, M. 2005, "Mikrokleben", in Montage hybrider Mikrosysteme: Handhabungs- und Fügetechniken für die Klein- und Mittelserienfertigung, Hrsg. Dilthey, U. \& Brandenburg, A., Springer, Berlin, Heidelberg, New York, Kapitel 3.9, S. 161-172, ISBN 3540237062.

DIN 863-1, Meßschrauben: Teil 1: Bügelmeßschrauben, Normalausführung, Deutsches Institut für Normung e. V., Stand April 1999.

DIN 8580, Fertigungsverfahren: Begriffe, Einteilung, Deutsches Institut für Normung e. V., Stand September 2003.

DIN 8589-11, Fertigungsverfahren Spanen: Teil 11: Schleifen mit rotierendem Werkzeug Einordnung, Unterteilung, Begriffe, Deutsches Institut für Normung e. V., Stand September 2003.

DIN 8590, Fertigungsverfahren Abtragen: Einordnung, Unterteilung, Begriffe, Deutsches Institut für Normung e. V., Stand September 2003. 
DIN 8593-0, Fertigungsverfahren Fügen: Teil 0: Allgemeines, Einordnung, Unterteilung, Begriffe, Deutsches Institut für Normung e. V., Stand September 2003.

DIN 32540, Laserstrahlabtragen - Thermisches Abtragen mit dem Laserstrahl - Begriffe, Einflussgrößen, Durchführung, Deutsches Institut für Normung e. V., Stand August 2012.

DIN 32564-1, Fertigungsmittel für Mikrosysteme - Begriffe - Teil 1: Allgemeine Begriffe der Mikrosystemtechnik, Deutsches Institut für Normung e. V., Stand August 2012.

DIN 32564-2, Fertigungsmittel für Mikrosysteme - Begriffe - Teil 2: Basistechnologien und Herstellung, Deutsches Institut für Normung e. V., Stand Mai 2004.

DIN EN 13887, Strukturklebstoffe: Leitlinien für die Oberflächenvorbehandlung von Metallen und Kunststoffen vor dem Kleben, Deutsches Institut für Normung e. V., Stand November 2003.

DIN EN ISO 14644-1, Reinräume und zugehörige Reinraumbereiche: Teil 1: Klassifizierung der Luftreinheit, Deutsches Institut für Normung e. V., Stand Juli 1999.

Ehrfeld, W. 2002, Handbuch Mikrotechnik, Carl Hanser Verlag, München, ISBN 3446215069.

Eichler, J. und Eichler, H. J. 2006, Laser: Bauformen, Strahlführung, Anwendungen, Springer, Berlin, Heidelberg, New York, ISBN 9783540301493.

Eiler, K. L. 2011, Aufbau eines manuellen Montageplatzes zur Verbindung zweier Mikrobauteile, Semesterarbeit, Technische Universität München.

ElringKlinger Kunststofftechnik 2014, Werkstoffe und Halbzeuge aus PTFE. Der außergewöhnliche Werkstoff für maßgeschneiderte Funktionalität, ElringKlinger Kunststofftechnik, S. 1-44. Verfügbar unter: <http://www.elringklinger-kunststoff.de/ fileadmin/user_upload/pdf/service/katalog-downloads/3Werkstoffe_u_Halbzeuge>. [24.01.2015].

Elsner, P. C. 2009, 3D-Drucktechnologie - Grundlagen zur Herstellung polymerer Bauteile mit gradierten Werkstoffeigenschaften, Dissertation, Technischen Universität Berlin.

Fatikow, S. 2000, Mikroroboter und Mikromontage: Aufbau, Steuerung und Planung von flexiblen mikroroboterbasierten Montagestationen, B. G. Teubner Verlag, Stuttgart, Leipzig, ISBN 9783519062646.

Festo 2014, Grundlagen der Vakuumtechnik, Festo, S. 1-54. Verfügbar unter: <http://www.festo.com/net/SupportPortal/Files/9916/Grundlagen_Vakuumtechnik.pdf>. [24.01.2015].

Fischer, U., Gomeringer, R., Heinzler, M., Kilgus, R., Näher, F., Oesterle, S., Paetzold, H. und Stephan, A. 2008, Tabellenbuch Metall, Verlag Europa Lehrmittel, Haan-Gruiten, ISBN 9783808517246.

Franssila, S. 2010, Introduction to Microfabrication, John Wiley \& Sons, Chichester, ISBN 9780470749838. 
Fujita, H. 2007, "Two decades of MEMS - from surprise to enterprise", Proceedings IEEE International Conference on Micro Electro Mechanical Systems (MEMS), Kobe, Japan, S. 1-6.

Fujita, S., Onuki-Nagasaki, R., Fukuda, J., Enomoto, J., Yamaguchi, S. und Miyake, M. 2013, "Development of super-dense transfected cell microarrays generated by piezoelectric inkjet printing", Lab Chip, vol. 13, no. 1, S. 77-80.

Gad-el-Hak, M. 2006, "Introduction", in MEMS: Introduction and Fundamentals, Hrsg. Gad-el-Hak, M., CRC Press, Boca Raton, Kapitel 1, S. 1/1-1/5, ISBN 0849391377.

Gaugel, T., Bengel, M. und Malthan, D. 2004, "Building a mini-assembly system from a technology construction kit", Assembly Automation, vol. 24, no. 1, S. 43-48.

Ge, Y., Gaines, J. A. und Nelson, B. J. 2005, "Optomechatronic Design of Microassembly Systems for Manufacturing Hybrid Microsystems", IEEE Transactions on Industrial Electronics, vol. 52, no. 4, S. 1013-1023.

Gerlach, G. und Dötzel, W. 2006, Einführung in die Mikrosystemtechnik: Ein Kursbuch für Studierende, Carl Hanser Verlag, München, ISBN 9783446225589.

Globisch, S. 2011, Lehrbuch Mikrotechnologie, Carl Hanser Verlag, München, ISBN 9783446425606.

Glück, M. 2005, MEMS in der Mikrosystemtechnik: Aufbau, Wirkprinzipien, Herstellung und Praxiseinsatz mikroelektromechanischer Schaltungen und Sensorsysteme, B. G. Teubner Verlag / GWV Fachverlage, Wiesbaden, ISBN 3519005204.

Greitmann, G. 1998, Micromechanical Tactile Gripper System for Micro Assembly, Dissertation, Eidgenössische Technische Hochschule Zürich.

Günther, D. 2008, Bimorph-Piezoaktoren mit strukturierten Elektroden für die Mikrofluidik, Dissertation, Technische Universität München.

Habenicht, G. 2009, Kleben: Grundlagen, Technologien, Anwendungen, Springer-Verlag, Berlin, Heidelberg, ISBN 9783540852643.

Häcker Automation, Modulare Automatisierungslösungen. Verfügbar unter: <http://www.haecker-automation.de/produkte/plattformen.html>. [03.09.2014].

Hagedorn, L., Thonfeld, W. und Rankers, A. 2009, Konstruktive Getriebelehre, Springer, Heidelberg, Dordrecht, London, New York, ISBN 9783642016134.

Hartzell, A. L., da Silva, M. G. und Shea, H. R. 2011, MEMS Reliability, Springer, New York, Dordrecht, Heidelberg, London, ISBN 9781441960177.

Heinzl, J. 2008, "Ink Jets", in Comprehensive Microsystems, Hrsg. Gianchandani, Y. B., Tabata, O. \& Zappe, H., Elsevier, Amsterdam, Kapitel 3.11, S. 335-368, ISBN 9780444521903. 
Herz, M., Horsch, D., Storch, R., Wackerle, M., Lueth, T. und Richter, M. 2010, "Modeling, Fabrication and Characterization of a High-Performance Micropump", Proceedings IEEE 23rd International Conference on Micro Electro Mechanical Systems (MEMS), Hongkong, China, S. 1083-1086.

Hesse, S. 2012, "Automatische Montagemaschinen", in Montage in der industriellen Produktion: Ein Handbuch für die Praxis, Hrsg. Lotter, B. \& Wiendahl, H.-P., Springer-Verlag, Berlin, Heidelberg, Kapitel 8, S. 195-272, ISBN 9783642290602.

Hilleringmann, U. 2006, Mikrosystemtechnik: Prozessschritte, Technologien, Anwendungen, B. G. Teubner Verlag / GWV Fachverlage, Wiesbaden, ISBN 3835100033.

Hines, D. R., Siwak, N. P., Mosher, L. A. und Ghodssi, R. 2011, "MEMS Lithography and Micromachining Techniques", in MEMS Materials and Process Handbook, Hrsg. Ghodssi, R. \& Lin, P., Springer, New York, Dordrecht, Heidelberg, London, Kapitel 9, S. 667-754, ISBN 9780387473161.

Höhn, M. 2001, Sensorgeführte Montage hybrider Mikrosysteme, Band 149, Forschungsberichte IWB, Herbert Utz Verlag, München, ISBN 3831600120.

Hölz, H., Mollenhauer, K. und Tschöke, H. 2011, "Kolbenmaschinen", in Dubbel: Taschenbuch für den Maschinenbau, Hrsg. Grote, K.-H. \& Feldhusen, J., SpringerVerlag, Berlin, Heidelberg, ISBN 9783642173059.

Hsu, T. R. und Clatterbaugh, J. 2004, "Joining and bonding technologies", in MEMS Packaging, Hrsg. Hsu, T. R., INSPEC, London, Kapitel 2, S. 23-59, ISBN 0863413358.

Hüdig, M. 2014, Erweiterung eines Mikromontageplatzes mit einer Rakeleinheit für den homogenen und definierten Klebstoffauftrag auf Substraten, Semesterarbeit, Technische Universität München.

Huff, M. A., Bart, S. F. und Lin, P. 2011, "MEMS Process Integration", in MEMS Materials and Process Handbook, Hrsg. Ghodssi, R. \& Lin, P., Springer, New York, Dordrecht, Heidelberg, London, Kapitel 14, S. 1045-1181, ISBN 9780387473161.

Hwang, H., Kang, G., Yeon, J. H., Nam, Y. und Park, J. K. 2009, "Direct rapid prototyping of PDMS from a photomask film for micropatterning of biomolecules and cells", Lab Chip, vol. 9, no. 1, S. 167-170.

Jia, G. und Madou, M. J. 2006, "MEMS Fabrication", in MEMS: Design and Fabrication, Hrsg. Gad-el-Hak, M., CRC Press, Boca Raton, Kapitel 3, S. 3/1-3/214, ISBN 9780849391385.

Kagerer, M., Eiler, K. L., Irlinger, F. und Lueth, T. C. 2011, "Development and Application of a Low-Cost Manual Micro Assembly System with Integrated Heater", Proceedings IEEE International Conference on Robotics and Biomimetics (ROBIO), Phuket, Thailand, S. 2963-2968.

Kagerer, M., Irlinger, F. und Lueth, T. C. 2011, "Laser Source Independent Basic Parameters in Micro-Cutting", Proceedings IEEE / ASME International Conference on Advanced Intelligent Mechatronics (AIM), Budapest, Ungarn, S. 391-396. 
Kagerer, M., Eiler, K. L., Irlinger, F. und Lueth, T. C. 2012a, "Intelligent Combination of Batch Fabrication with Rapid Prototyping Techniques for a Drop-on-Demand Microdrop Generator", Proceedings ASME International Mechanical Engineering Congress and Exhibition (IMECE), Houston, USA, S. 619-628.

Kagerer, M., Eiler, K. L., Ottnad, T., Irlinger, F. und Lueth, T. C. 2012b, "Piezo Inkjet Dropon-Demand Experimentation Platform Manufactured with Rapid Prototyping Techniques Enabling Future Technologies", Proceedings IEEE International Conference on Robotics and Biomimetics (ROBIO), Guangzhou, China, S. 1244-1249.

Kagerer, M., Irlinger, F. und Lueth, T. C. 2012, "Laser Source Independent Basic Parameters - Focus Position, Pulse Overlap, Track Overlap - in Laser Micro Milling Using as Rapid Manufacturing Process", Proceedings IEEE / ASME International Conference on Advanced Intelligent Mechatronics (AIM), Kaohsiung, Taiwan, S. 135-140.

Kagerer, M., Eiler, K. L., Ottnad, T., Irlinger, F. und Lueth, T. C. 2013a, "Mikrotropfenerzeuger Hergestellt mit Rapid Manufacturing Verfahren", Proceedings VDE MikroSystemTechnik Kongress, Aachen, Deutschland, S. 345-348.

Kagerer, M., Huedig, M., Lueth, T. C. und Irlinger, F. 2013b, "Manual Microassembly System with Integrated Squeegee Device for Homogenous and Defined Adhesive Layers for Bimorph Piezoelectric Actuators Using in Drop-on-Demand Techniques", Proceedings IEEE International Conference on Robotics and Biomimetics (ROBIO), Shenzhen, China, S. 1911-1917.

Kagerer, M., Rumschoettel, D., Ottnad, T., Lueth, T. C. und Irlinger, F. 2013c, "Fast Droplet Generation with a Printhead Manufactured with Rapid Manufacturing Techniques Mounted on a Carrier Board", Proceedings ASME International Mechanical Engineering Congress \& Exposition (IMECE), San Diego, USA, S. 1-8 (Paper Nummer: IMECE2013-63147).

Kagerer, M., Meeuw, A., Berger, J., Rumschoettel, D., Lueth, T. C. und Irlinger, F. 2014, "Relevant Influencing Factors on Droplet Characteristics for a Piezoelectrically Driven Drop-on-Demand Printhead", Proceedings ASME International Mechanical Engineering Congress \& Exposition (IMECE), Montréal, Canada, S. 1-10 (Paper Nummer: IMECE2014-36199).

Kim, C. S., Sim, W., Lee, J. S., Yoo, Y.-S. und Joung, J. 2010, "Design and Characterization of Piezoelectric Inkjet for Micro Patterning of Printed Electronics", Proceedings IEEE International Symposium on Industrial Electronics (ISIE), Bari, Italien, S. 1817-1822.

Klocke Nanotechnik, 15.03.2013, From Microassembly to Micro Production Systems. Verfügbar unter: <http://www.nanomotor.de/n_microassembly.htm>. [02.09.2014].

Ko, S. H., Chung, J., Hotz, N., Nam, K. H. und Grigoropoulos, C. P. 2010, "Metal nanoparticle direct inkjet printing for low-temperature 3D micro metal structure fabrication", Journal of Micromechanics and Microengineering, vol. 20, no. 12, S. 1-7.

Krebs, F. C. 2009, "Fabrication and processing of polymer solar cells: A review of printing and coating techniques", Solar Energy Materials and Solar Cells, vol. 93, no. 4, S. 394-412. 
Läßiger, B. 1995, Kontroliierter Formabtrag durch Sublimation mittels Laserstrahlung, Shaker Verlag, Aachen, ISBN 9783826504532.

Lawes, R. A. 2007, "Manufacturing costs for microsystems/MEMS using high aspect ratio microfabrication techniques", Microsystem Technologies, vol. 13, no. 1, S. 85-95.

Lazar, I. M., Grym, J. und Foret, F. 2006, "Microfabricated devices: a new sample introduction approach to mass spectrometry", Mass Spectrometry Reviews, vol. 25, no. 4 , S. 573-594.

Lee, E. R. 2003, Microdrop Generation, CRC Press, Boca Raton, ISBN 084931559X.

Lemmermeyer, B. 2006, Ein hochtemperaturbeständiger Einzeltropfenerzeuger für flüssige Metalle, Reihe 1, Nr. 392, Fortschritt-Berichte, VDI Verlag, Düsseldorf, ISBN 3183392011.

Liess, H.-A. 2006, Untersuchung von Laser-, Funkenerosions- und Räumverfahren zur Herstellung von zykloidischen Mikroverzahnungen, Reihe 2, Nr. 657, FortschrittBerichte, VDI Verlag, Düsseldorf, ISBN 3183657023.

Livelo, E. und Rojas, R. P. V. 2008, "Microdot Epoxy Stamping Process Qualification For Substrate Based Optocoupler BGA Packaging", Proceedings IEEE Electronics Packaging Technology Conference (EPTC), Singapur, S. 1131-1136.

Lotter, B. 2012, "Einführung", in Montage in der industriellen Produktion: Ein Handbuch für die Praxis, Hrsg. Lotter, B. \& Wiendahl, H.-P., Springer-Verlag, Berlin, Heidelberg, Kapitel 1, S. 1-8, ISBN 9783642290602.

Lyshevski, S. E. 2005, Nano- and Micro-Electromechanical Systems: Fundamentals of Nanoand Microengineering, CRC Press, Boca Raton, ISBN 9780849328381.

Madou, M. J. 2002, Fundamentals of Microfabrication: The Science of Miniaturization, CRC Press, Boca Raton, ISBN 0849308267.

MARTIN, Rework Station EXPERT 04.6. Verfügbar unter: <http://www.martinsmt.de/rework/products/reworkplatz-expert-046.html>. [02.09.2014].

Meeuw, A. 2014, Beschreibung des Einflusses der elektrischen Ansteuerung und der Düsengeometrie eines Mikrotropfenerzeugers auf den Tropfenbildungsprozess, Semesterarbeit, Technische Universität München.

Meijer, J. 2004, "Laser beam machining (LBM), state of the art and new opportunities", Journal of Materials Processing Technology, vol. 149, no. 1-3, S. 2-17.

Menz, W., Mohr, J. und Paul, O. 2005, Mikrosystemtechnik für Ingenieure, Wiley-VCH Verlag, Weinheim, ISBN 9783527305360.

minitron elektronik 2014, microkerf - Dicing Blades für 2"-Spindeln, minitron elektronik, S. 1-12. Verfügbar unter: <http://minitron.com/uploads/media/dicblae.pdf >. [24.01.2015]. 
Moon, K. S., Choi, J. H., Choi, D.-J., Kim, S. H., Ha, M. H., Nam, H.-J. und Kim, M. S. 2008, "A new method for analyzing the refill process and fabrication of a piezoelectric inkjet printing head for LCD color filter manufacturing", Journal of Micromechanics and Microengineering, vol. 18, no. 12, S. 1-13.

Moore, M. J., Friedman, J. A., Lewellyn, E. B., Mantila, S. M., Krych, A. J., Ameenuddin, S., Knight, A. M., Lu, L., Currier, B. L., Spinner, R. J., Marsh, R. W., Windebank, A. J. und Yaszemski, M. J. 2006, "Multiple-channel scaffolds to promote spinal cord axon regeneration", Biomaterials, vol. 27, no. 3, S. 419-29.

Nienhaus, M. 1999, Zur Montage hybrider Mikrosysteme am Beispiel von Radarsensoren und Umlaufrädergetrieben, Reihe 2, Nr. 501, Fortschritt-Berichte, VDI Verlag, Düsseldorf, ISBN 3183501023.

Noguera, R., Lejeune, M. und Chartier, T. 2005, "3D fine scale ceramic components formed by ink-jet prototyping process", Journal of the European Ceramic Society, vol. 25, no. 12 , S. 2055-2059.

Ottnad, T., Irlinger, F. und Lueth, T. C. 2011, "Design, construction, and verification of a printhead - tolerant towards bubbles - dosing liquid wax using rapid prototyping techniques", Proceedings IEEE / ASME International Conference on Advanced Intelligent Mechatronics (AIM), Budapest, Ungarn, S. 215-219.

Ottnad, T., Kagerer, M., Irlinger, F. und Lueth, T. C. 2012, "Modification and Further Development of a Drop on Demand Printhead for Wax enabling Future 3D-Printing and Rapid Prototyping", Proceedings IEEE / ASME International Conference on Advanced Intelligent Mechatronics (AIM), Kaohsiung, Taiwan, S. 117-122.

Parashkov, R., Becker, E., Riedl, T., Johannes, H. H. und Kowalsky, W. 2005, "Large Area Electronics Using Printing Methods", Proceedings IEEE, vol. 93, no. 7, S. 1321-1329.

Petersen, B. 2003, Flexible Handhabungstechnik für die automatisierte Mikromontage, Berichte aus der Produktionstechnik, Bd. 2003, 17, Shaker Verlag, Aachen, ISBN 3832218157.

Petzold, B. 2008, Entwicklung eines Operatorarbeitsplatzes für die telepräsente Mikromontage, Band 217, Forschungsberichte IWB, Herbert Utz Verlag, München, ISBN 9783831608058.

Pfeifer, G. 1982, Piezoelektrische lineare Stellantriebe, Wissenschaftliche Schriftenreihe der Technischen Hochschule Karl-Marx-Stadt 6 / 1982, Chemnitz, ISSN 0323-6374.

Pimpin, A. und Srituravanich, W. 2011, "Review on Micro- and Nanolithography Techniques and Their Applications", Engineering Journal, vol. 16, no. 1, S. 37-55.

Raatz, A., Löchte, C., Burisch, A., Wrege, J. und Hesselbach, J. 2012, "Mikromontage", in Montage in der industriellen Produktion: Ein Handbuch für die Praxis, Hrsg. Lotter, B. \& Wiendahl, H.-P., Springer-Verlag, Berlin, Heidelberg, Kapitel 17, S. 443-472, ISBN 9783642290602.

Rebello, K. J. 2004, "Applications of MEMS in surgery", Proceedings IEEE, vol. 92, no. 1, S. 43-55. 
Rumschoettel, D., Kagerer, M., Irlinger, F. und Lueth, T. C. 2014, "Compact Model for the Static and Dynamic Behavior of a Piezoelectric Bimorph Actuator for Microfluidic MEMS", Proceedings ASME International Mechanical Engineering Congress \& Exposition (IMECE), Montréal, Canada, S. 1-9 (Paper Nummer: IMECE2014-36654).

Scheicher, R. 2004, Kompaktmodell zur Systemsimulation eines Tropfenerzeugers mit Piezobiegewandlern, Reihe 8, Nr. 1053, Fortschritt-Berichte, VDI Verlag, Düsseldorf, ISBN 3185053087.

Scheuenpflug, M. 2011, Passive Rapid-Prototyping-Mikromischer für die Synthese von Radiopharmaka, Reihe 17, Nr. 282, Fortschritt-Berichte, VDI Verlag, Düsseldorf, ISBN 9783183282173.

Schilp, J. 2012, Adaptive Montagesysteme für hybride Mikrosysteme unter Einsatz von Telepräsenz, Band 244, Forschungsberichte IWB, Herbert Utz Verlag, München, ISBN 9783831640638.

Schilp, M. 2001, "Dosier- und Dispenstechnik - das Problem des kleinen Tropfens", in Seminarberichte 59 IWB: Automatisierte Mikromontage - Werkzeuge und Fügetechnologien für die Mikrosystemtechnik, Hrsg. Reinhart, G., Herbert Utz Verlag, München, ISBN 3896750593.

Schneider, J. (Auftragssachbearbeitung DISCO HI-TEC Europe) 2015, Persönliches Gespräch bzgl. Anschaffungskosten von Wafersäge DAD321. [23.04.2015].

Schwesinger, N. (Leiter des Fachgebietes MMS an der TU München) 2015, Persönliches Gespräch bzgl. Anschaffungskosten mikroelektronischer Gerätschaften. [21.04.2015].

Schwesinger, N., Dehne, C. und Adler, F. 2009, Lehrbuch Mikrosystemtechnik: Anwendungen, Grundlagen, Materialien und Herstellung von Mikrosystemen, Oldenbourg Wissenschaftsverlag, München, ISBN 9783486579291.

Seckner, M. 2008, Unterstützung automatisierter Mikroproduktion durch wandlungsfähige Montageanlagen - Konzeption und Realisierung einer flexiblen und reaktiven Mikromontage, Band 19, Fortschritt-Berichte pak, Technische Universität Kaiserslautern, Kaiserslauten, ISBN 9783939432913.

Seitz, H. und Heinzl, J. 2004, "Modelling of a microfluidic device with piezoelectric actuators", Journal of Micromechanics and Microengineering, vol. 14, no. 8, S. 1140-1147.

Smith, C. S. 1954, "Piezoresistance Effect in Germanium and Silicon", Physical Review, vol. 94, no. 1, S. 42-49.

Sumerel, J., Lewis, J., Doraiswamy, A., Deravi, L. F., Sewell, S. L., Gerdon, A. E., Wright, D. W. und Narayan, R. J. 2006, "Piezoelectric ink jet processing of materials for medical and biological applications", Biotechnology Journal, vol. 1, no. 9, S. 976-987.

Sumner, A. L., Menke, E. J., Dubowski, Y., Newberg, J. T., Penner, R. M., Hemminger, J. C., Wingen, L. M., Brauers, T. und Finlayson-Pitts, B. J. 2004, "The nature of water on surfaces of laboratory systems and implications for heterogeneous chemistry in the troposphere", Physical Chemistry Chemical Physics, vol. 6, no. 3, S. 604-613. 
Sun, J., Fuh, J. Y. H., Thian, E. S., Hong, G. S., Wong, Y. S., Yang, R. und Tan, K. K. 2013, "Fabrication of electronic devices with multi-material drop-on-demand dispensing system", International Journal of Computer Integrated Manufacturing, vol. 26, no. 10, S. $897-906$.

Tadigadapa, S. und Lärmer, F. 2011, "Dry Etching for Micromachining Applications", in MEMS Materials and Process Handbook, Hrsg. Ghodssi, R. \& Lin, P., Springer, New York, Dordrecht, Heidelberg, London, Kapitel 7, S. 403-456, ISBN 9780387473161.

Tadigadapa, S. A. und Najafi, N. 2003, "Developments in Microelectromechanical Systems (MEMS): A Manufacturing Perspective", Journal of Manufacturing Science and Engineering, vol. 125, no. 4, S. 816-823.

Tseng, F.-G. 2006, "Microdroplet Generators", in MEMS: Applications, Hrsg. Gad-el-Hak, M., CRC Press, Boca Raton, Kapitel 10, S. 10/1-10/33, ISBN 0849391393.

UHU 2015, UHU Technisches Merkblatt: UHU PLUS ENDFEST 300, S. 1-6. Verfügbar unter: < http://www.uhu-profi.de/uploads/tx_ihtdatasheets/tds_plus_ endfest300.pdf >. [22.04.2015].

VDI 2860, Montage- und Handhabungstechnik: Handhabungsfunktionen, Handhabungseinrichtungen; Begriffe, Definitionen, Symbole, VDI Verein Deutscher Ingenieure, Stand August 2012.

Völklein, F. und Zetterer, T. 2006, Praxiswissen Mikrosystemtechnik: Grundlagen, Technologien, Anwendungen, Friedr. Vieweg \& Sohn Verlag / GWV Fachverlage, Wiesbaden, ISBN 3528138912.

Wijshoff, H. 2010, "The dynamics of the piezo inkjet printhead operation", Physics Reports, vol. 491, no. 4-5, S. 77-177.

Wolf, R. 2003, Rapid Prototyping in der Mikrotechnik mittels Laserablation, Reihe 2, Nr. 634, Fortschritt-Berichte, VDI Verlag, Düsseldorf, ISBN 3183634023.

Yeon, J. H. und Park, J.-K. 2007, "Microfluidic Cell Culture Systems for Cellular Analysis", Biochip Journal, vol. 1, no. 1, S. 17-27.

Zäh, M. F. 2006, Wirtschaftliche Fertigung mit Rapid-Technologien: Anwender-Leitfaden zur Auswahl geeigneter Verfahren, Carl Hanser Verlag München, Wien, ISBN 9783446439160.

Zeilinger, T. 2013, Laserbasierte Bauteillagebestimmung bei der Montage optischer Mikrokomponenten, Band 269, Forschungsberichte IWB, Herbert Utz Verlag, München, ISBN 9783831642342.

Zorman, C. A. und Mehregany, M. 2006, "Materials for Microelectromechanical Systems", in MEMS: Design and Fabrication, Hrsg. Gad-el-Hak, M., CRC Press, Boca Raton, Kapitel 2, S. 2/1-2/26, ISBN 0849391385. 


\section{Publikationen}

Kagerer, M., Behlert, R., Irlinger, F. und Lueth, T. C. 2011, "New approach in MEMS integration with UV laser micro-cutting", Proceedings International Conference of the European Society for Precision Engineering \& Nanotechnology, Comersee, Italien, S. 140-143.

Kagerer, M., Behlert, R., Irlinger, F. und Lueth, T. C. 2011, "Laser-Mikroschneiden von schrägen Durchkontaktierungen", Proceedings VDE MikroSystemTechnik Kongress, Darmstadt, Deutschland, S. 539-542.

Kagerer, M., Eiler, K. L., Irlinger, F. und Lueth, T. C. 2011, "Development and Application of a Low-Cost Manual Micro Assembly System with Integrated Heater", Proceedings IEEE International Conference on Robotics and Biomimetics (ROBIO), Phuket, Thailand, S. 2963-2968.

Kagerer, M., Irlinger, F. und Lueth, T. C. 2011, "Laser Source Independent Basic Parameters in Micro-Cutting", Proceedings IEEE / ASME International Conference on Advanced Intelligent Mechatronics (AIM), Budapest, Ungarn, S. 391-396.

Kagerer, M., Rumschoettel, D., Irlinger, F. und Lueth, T. C. 2011, "Fabrication and Application of a Chemical Resistant Low-Cost Microdrop Generator", Proceedings ASME International Mechanical Engineering Congress and Exhibition (IMECE), Denver, USA, S. 601-610.

Kagerer, M., Behlert, R., Irlinger, F. und Lüth, T. C. 2012, "Laser-Mikroschneiden von Durchkontaktierungen unter frei definierbaren Winkeln", Proceedings 3. Landshuter Symposium Mikrosystemtechnik, Landshut, Deutschland, S. 67-74.

Kagerer, M., Eiler, K. L., Irlinger, F. und Lueth, T. C. 2012, "Intelligent Combination of Batch Fabrication with Rapid Prototyping Techniques for a Drop-on-Demand Microdrop Generator", Proceedings ASME International Mechanical Engineering Congress and Exhibition (IMECE), Houston, USA, S. 619-628.

Kagerer, M., Eiler, K. L., Ottnad, T., Irlinger, F. und Lueth, T. C. 2012, "Piezo Inkjet Dropon-Demand Experimentation Platform Manufactured with Rapid Prototyping Techniques Enabling Future Technologies", Proceedings IEEE International Conference on Robotics and Biomimetics (ROBIO), Guangzhou, China, S. 1244-1249.

Kagerer, M., Irlinger, F. und Lueth, T. C. 2012, "Laser Source Independent Basic Parameters - Focus Position, Pulse Overlap, Track Overlap - in Laser Micro Milling Using as Rapid Manufacturing Process", Proceedings IEEE / ASME International Conference on Advanced Intelligent Mechatronics (AIM), Kaohsiung, Taiwan, S. 135-140.

Kagerer, M., Eiler, K. L., Ottnad, T., Irlinger, F. und Lueth, T. C. 2013, "Mikrotropfenerzeuger Hergestellt mit Rapid Manufacturing Verfahren", Proceedings VDE MikroSystemTechnik Kongress, Aachen, Deutschland, S. 345-348. 
Kagerer, M., Heller, B., Lueth, T. C. und Irlinger, F. 2013, "Optimization of the ElectroMechanical Behavior of a Bimorph Piezoelectric Actuator for Drop-on-Demand Techniques Based on Finite Element Method", Proceedings ASME International Mechanical Engineering Congress \& Exposition (IMECE), San Diego, USA, S. 1-7 (Paper Nummer: IMECE2013-63150).

Kagerer, M., Huedig, M., Lueth, T. C. und Irlinger, F. 2013, "Manual Microassembly System with Integrated Squeegee Device for Homogenous and Defined Adhesive Layers for Bimorph Piezoelectric Actuators Using in Drop-on-Demand Techniques", Proceedings IEEE International Conference on Robotics and Biomimetics (ROBIO), Shenzhen, China, S. 1911-1917.

Kagerer, M., Rumschoettel, D., Ottnad, T., Lueth, T. C. und Irlinger, F. 2013, "Fast Droplet Generation with a Printhead Manufactured with Rapid Manufacturing Techniques Mounted on a Carrier Board", Proceedings ASME International Mechanical Engineering Congress \& Exposition (IMECE), San Diego, USA, S. 1-8 (Paper Nummer: IMECE2013-63147).

Kagerer, M., Meeuw, A., Berger, J., Rumschoettel, D., Lueth, T. C. und Irlinger, F. 2014, "Relevant Influencing Factors on Droplet Characteristics for a Piezoelectrically Driven Drop-on-Demand Printhead", Proceedings ASME International Mechanical Engineering Congress \& Exposition (IMECE), Montréal, Canada, S. 1-10 (Paper Nummer: IMECE2014-36199).

Ottnad, T., Kagerer, M., Irlinger, F. und Lueth, T. C. 2012, "Modification and Further Development of a Drop on Demand Printhead for Wax enabling Future 3D-Printing and Rapid Prototyping", Proceedings IEEE / ASME International Conference on Advanced Intelligent Mechatronics (AIM), Kaohsiung, Taiwan, S. 117-122.

Rumschoettel, D., Kagerer, M., Irlinger, F. \& Lueth, T. C. 2015, "Kompaktmodell für die Charakterisierung eines piezoelektrischen Membrandruckkopfes", Proceedings VDE MikroSystemTechnik Kongress, Karlsruhe, Deutschland, S. 1-4 (Paper Nummer: 1570106537).

Rumschoettel, D., Kagerer, M., Irlinger, F. und Lueth, T. C. 2014, "Compact Model for the Characterization of a Piezoelectric Bend-Mode Droplet Generator", Proceedings IEEE International Conference on Robotics and Biomimetics (ROBIO), Bali, Indonesien, S. 2582-2587.

Rumschoettel, D., Kagerer, M., Irlinger, F. und Lueth, T. C. 2014, "Compact Model for the Static and Dynamic Behavior of a Piezoelectric Bimorph Actuator for Microfluidic MEMS", Proceedings ASME International Mechanical Engineering Congress \& Exposition (IMECE), Montréal, Canada, S. 1-9 (Paper Nummer: IMECE2014-36654).

Schwaiger, J., Kagerer, M., Traeger, M., Gillen, S., Dobritz, M., Kleeff, J., Feussner, H. und Lueth, T. C. 2012, "Manufacturing of patient-specific pancreas models for surgical resections", Proceedings IEEE International Conference on Robotics and Biomimetics (ROBIO), Guangzhou, China, S. 991-998. 


\section{Betreute studentische Arbeiten}

Albrecht, J. 2012, Inbetriebnahme einer Positioniereinheit mit Erstellung eines bedienerfreundlichen Interfaces für ein Lasersystem, Semesterarbeit, Technische Universität München.

Behlert, R. 2011, Aufbau einer Anordnung für die Erzeugung von Durchkontaktierungen mit beliebigen Winkeln, Semesterarbeit, Technische Universität München.

Berger, J. 2014, Herstellung und Charakterisierung von Fluidgemischen mit definierten Eigenschaften und deren Einsatz in einem Mikrotropfenerzeuger, Semesterarbeit, Technische Universität München.

Eiler, K. L. 2011, Aufbau eines manuellen Montageplatzes zur Verbindung zweier Mikrobauteile, Semesterarbeit, Technische Universität München.

Eiler, K. L. 2012, Optimierung eines piezoelektrischen Drop-on-Demand Mikrotropfenerzeugers, Diplomarbeit, Technische Universität München.

Heller, B. 2013, Modellierung des elektromechanischen Verhaltens eines bimorphen Aktors für einen Mikrotropfenerzeuger, Semesterarbeit, Technische Universität München.

Hüdig, M. 2014, Erweiterung eines Mikromontageplatzes mit einer Rakeleinheit für den homogenen und definierten Klebstoffauftrag auf Substraten, Semesterarbeit, Technische Universität München.

Meeuw, A. 2012, Charakterisierung des elektro-mechanischen Verhaltens eines piezoelektrischen Wandlers für einen Piezomembrandruckkopf, Bachelorarbeit, Technische Universität München.

Meeuw, A. 2014, Beschreibung des Einflusses der elektrischen Ansteuerung und der Düsengeometrie eines Mikrotropfenerzeugers auf den Tropfenbildungsprozess, Semesterarbeit, Technische Universität München.

Rumschöttel, D. 2012, Herstellung und Charakterisierung eines chemikalienresistenten planaren Mikrotropfenerzeugers, Semesterarbeit, Technische Universität München.

Rumschöttel, D. 2014, Kompaktmodell zur Charakterisierung eines piezoelektrisch betriebenen Mikrotropfenerzeugers, Diplomarbeit, Technische Universität München. 